\title{
Proceedings of the Fourth International American Moroccan Agricultural Sciences Conference - AMAS Conference IV, May 9-11, 2018, Meknes, Morocco
}

My Abdelmajid Kassem ${ }^{*}$, Alan Walters², Karen Midden², and Khalid Meksem²

1 Plant Genetics, Genomics, and Biotechnology Lab, Dept. of Biological Sciences, Fayetteville State University, Fayetteville, NC 28301, USA; ${ }^{2}$ Dept. of Plant, Soil, and Agricultural Systems, Southern Illinois University, Carbondale, IL 62901-4415, USA

Received: May 12, 2018 / Accepted: June 9, 2018

\begin{abstract}
The International American Moroccan Agricultural Sciences Conference (AMAS Conference; www.amas-conference.org) is an international conference organized by the High Council of Moroccan American Scholars and Academics (HC-MASA; www.hc-masa.org) in collaboration with various universities and research institutes in Morocco. AMAS IV was held on May 9-11, 2018 in Meknes, Morocco. The current proceedings summarizes abstracts from 73 oral presentations and 168 posters that were presented during AMAS Conference IV.
\end{abstract}

Keywords: AMAS Conference, HC-MASA, Agricultural Sciences.

*Corresponding author: mkassem@uncfsu.edu
ORAL PRESENTATIONS ABSTRACTS WEDNESDAY \& THURSDAY MAY $9 \& 10,2018$

\section{Session I: Plant Health \& Production I (Plenary Session)}

Co-Chair: David Lightfoot, Southern Illinois University, USA

Co-Chair: Ikram Blilou, KAUST, Saudi Arabia

O1. Detection of QTL Underlying Seed Composition Traits in Soybean [Glycine max (L.) Merr.]

Masum Akond', Jiazheng Yuan', Shiming Liu', Stella K. Kantartzi ${ }^{2}$, Khalid Meksem², Nacer Bellaloui ${ }^{3}$, David A. Lightfoot ${ }^{2}$, and My Abdelmaiid Kassem ${ }^{1 *}$. 
1 Plant Genomics and Biotechnology Lab, Department of Biological Sciences, Fayetteville State University, Fayetteville, NC 28301, USA; ${ }^{2}$ Department of Plant, Soil and Agricultural Systems, Southern Illinois University, Carbondale, IL 62901, USA; 3 USDA-ARS, Crop Genetics Research Unit, 141 Experiment Station Road, P.O. Box 345, Stoneville, MS 38776, USA. *Corresponding and Presenting Author: mkassem@ uncfsu.edu.

\section{Abstract}

Improving seed composition and quality including protein, oil, and beneficial fatty and amino acids contents is an important goal of soybean farmers and breeders. This study's aim was to use the 'Hamilton' by 'Spencer' recombinant inbred line (RIL) population ( $\mathrm{H} \times \mathrm{S}, \mathrm{n}=93$ ) and map quantitative trait loci (QTL) for protein, oil, fatty and amino acids contents. The genetic linkage map was created using 5,376 single nucleotide polymorphism (SNP). A total of 13 QTL for the traits studies have mapped on 4 chromosomes (chr) of the soybean genome. Three major QTL have been mapped to a 7-13 cM region on chr6. One major QTL for oil content (qOILO01; LOD $=39.47$, R2 $=76 \%$ ) that explained approximately $76 \%$ of the total variation in oil content in this population; one major QTL for ALA (qALA001; LOD $=50.24$, R2 $=74 \%$ ) that explained approximately $74 \%$ of the total variation in ALA content in this population; and one major QTL for palmitic acid content (qPALO01; $L O D=2.71, R 2=21 \%$ ) that explained approximately $21 \%$ of palmitic acid content in this population. These firm QTLs cover the approach for soybean seed protein, amino acids, oil, and fatty acids contents breeding by marker-assisted selection. Keywords: Soybean; SNP Linkage Map; QTL; RIL; protein, oil; fatty acids; 'Hamilton'; 'Spencer'.

O2. Integrative Approach Toward Revealing and Understanding Complexity of Root System Architecture in Date Palm

$\underline{\text { Ikram Blilou* }}$

Plant Cell and Developmental Biology, Biological and Environmental Science and Engineering, King Abdullah University of Science and Technology, Thuwal, Saudi Arabia. *Corresponding and Presenting Au- thor: Ikram.blilou@kaust.edu.sa.

\section{Abstract}

The evolution from the primordial aquatic organisms to vascular terrestrial plants has been accompanied by increasing complexity in the structure and functions of their vegetative and reproductive organs. Plants have undergone dramatic changes in their root systems to adapt to terrestrial life. The development of complex diverse root architectures gave plants the advantage ability to colonize new and particularly arid and dry environments. Date palm Phoenix dactylifera fruits are known for their high nutritive, economic and social values. In arid and semi-arid areas, it plays an important role in affecting the microclimate by creating a microsystem allowing desert farming. Understanding the properties of growth and development in date palm is an essential step towards gaining insights as to how plants have evolved their strategies to cope with changes in their surrounding and survive in challenged habitats like the desert. To unravel the underlying mechanisms of date palm adaptation to desert conditions we conducted a detailed analysis of date palm anatomy during different stages of development from germination to adult plants. Using the art of state imaging technologies, we unraveled new developmental mechanisms in date palm occurring during germination, plant growth and development. MicroCT Xray imaging technology combined with high resolution microscopy revealed that date palm roots bear structures that have not been previously described. Some of these structures are conserved only among desert palm species. In addition, a comparative studies of date palm cultivars originated from different geographical habitat, Tunisia, UAE and KSA and having distinct levels of tolerance to soil salinity revealed substantial differences in root system architecture.

\section{O3. Enhanced Nutritional Quality By Glutamate Synthe- sized By Transgenes Improves Crop Growth, Herbicide Tolerance And Reduced Fungal Toxin Contamination.}

\section{David A. Lightfoot ${ }^{*}$.}

Department of Plant, Soil, and Agricultural Systems, Southern Illinois University, Carbondale, IL 62901, USA. "Corresponding and Presenting Author: ga4082@siu.edu. 


\section{Abstract}

Maize (Zea mays L.) and other crop plants have altered transcript and metabolic profiles caused by in planta expression of the bacterial glutamate dehydrogenase (GDH; EC 1.4.1.2), encoded by a modified gdhA. Labeling experiments showed glutamate produced in the cytoplasm was increased 2 fold, but only by $5 \%$ in the chloroplast. The change in glutamate concentration in the cytoplasmic pool has profound effects on metabolism and homeostasis. The metabolic changes resulted in phenotypic changes that included increases in; mean plant biomass production in dry soils; nutritional value; tolerance to the herbicide phosphinothricin; and tolerance to both severe and mild water deficit improved NUE and WUE. Crosses to waxy, flinty and brown-midrib maize were made. Comparisons of transgenic and non-transgenic maize showed a $11 \%$ increase in WUE and $9 \%$ increase in NUE across a range of water delivery rates. Resistance to rotting necrotrophs including carcinogenic Aspergillus flavus and Fusarium virguliforme was noted. Sporulation of A. flavus was inhibited. The abundance of 747 fungal and 395 maize grain transcripts were altered suggesting the GDH maize was not supporting normal fungal growth because of metabolic and compositional alterations. Cancer incidences due to toxin contamination can potentially be reduced by $50 \%$ by $\mathrm{GDH}$. Better nutrition will lead to less pollution.

\section{O4. The Rose Genome and Beyond; Understanding Rose Domestication and the Mechanisms Underlying Major Traits.}

\section{Mohammed Bendahmane*}

Ecole Normale Supérieure (INRA CNRS Lyon 1 ENSL), Lyon, France. ${ }^{*}$ Corresponding and Presenting Author: mohammed.bendahmane@ens-lyon.fr.

\section{Abstract}

Roses are of high symbolic value and have great cultural importance in many societies worldwide. They hold high cultural and economic importance as ornamental plants and for the perfume industry. The rose is well suited to be an original model organism for woody ornamental species as it has a relatively small genome size (560Mbp) and it has a short life cycle for a perennial woody plant. During centuries, generations of rose breeders had fastidiously selected the showy and desirable traits of Rosa species based on keen and meticulous observation. Several traits, involving mainly floral quality (ie. recurrent flowering, flower form and double flower formation, scent biosynthesis, ...), are of high economic importance, but the molecular and genetic mechanisms controlling these traits remain poorly understood1. During the past years, we generated a number of molecular and biotechnology tools such as reproducible genetic transformation as well as a database that provides useful information on Rosa sp. genome structure and expressed genes with thorough annotation and an overview of expression patterns for transcripts with good accuracy ${ }^{1-3}$. These tools allowed us to discover the molecular mechanisms controlling the doubleflower formation ${ }^{4}$ and the biosynthesis of major scent molecules, the terpenes 5 . Recently, we used Single Molecule Real-Time sequencing and an original meta-assembly approach to obtain a very high-quality assembly -at the chromosome level- for the rose cultivar Rosa chinensis, known to have extensively participated in rose breeding and the creation of modern roses6. Resequencing of the genome of 14 major genotypes that contributed to rose domestication, along with genome diversity analyses, highlighted the mosaic origin of the genome of modern rose hybrids that combines the growth vigor traits of European species and recurrent blooming of Chinese species6. Expert gene annotations along with gene expression data permitted the reconstruction of gene regulatory pathways associated with major rose traits, and allowed to describe epigenetic variation landscapes along the rose genome ${ }^{6}$. Reconstruction of regulatory and secondary metabolism pathways involved in scent and flower color, validated by biochemical and molecular analyses, allows us to propose models of interconnected regulation of flower color and scent compounds6. The data also provide indication on why roses evolved alternative routes to produce scent compounds, such as terpenes, in the petals. Together, these resources provide a solid foundation for understanding the mechanisms governing rose traits and their diversity and will accelerate improvement in roses, Rosaceae and ornamentals. Recent advances will be presented and discussed. 


\section{Selected References}

1. Bendahmane et al., (2013) Genetics and genomics of flower initiation and development in roses. J Exp Bot 64(4):847-57.

2. Dubois et al., (2012) Transcriptome database resource and gene expression atlas for the rose. BMC Genomics 13:638.

3. Dubois et al., (2011) Genomic Approach to Study Floral Development Genes in Rosa sp. PLoS ONE 6: e28455.

4. Dubois et al., (2010). Tinkering with the C-function: A molecular frame for the selection of double flowers in cultivated roses. PLoS ONE 5: e9288

5. Magnard et al., (2015) Biosynthesis of monoterpene scent compounds in roses. Science 349: 81-3.

6. Raymond et al., The Rosa genome provides new insights into the domestication of modern roses. Nature Genetics (in press).

\section{O5. Valorization of Moroccan Saffron through Scientific Research}

Serghini Mohammed Amine ${ }^{*}$, K. Lagram, M. Ben El Caid, L.H. Atyane, M. Lachheb, L. Salaka, and S. El Merzougui

Laboratory of Biotechnology and Valorization of Natural Ressources, Faculty of Sciences, Ibn Zohr University, Agadir, Morocco. "Corresponding and Presenting Author: maserghini@live.fr.

\section{Abstract}

Saffron is the most expensive spice in the world. Also called 'red gold', this spice is derived from the dried red stigmas of Crocus sativus L. It is well appreciated for its color (crocin), taste (picrocrocin) and aroma (safranal). It has medicinal properties at low doses. Recently, a growing interest focuses on its components which may have an anti-carcinogenic effect. World saffron production amounts to some 320 tonnes/ year. The main producing countries are: Iran (94\%), Greece (2.2\%), Morocco (1.5\%), India (1\%), Spain $(0.5 \%)$ and other countries $(0.7 \%)$. Morocco is the largest African producer of saffron with an annual turnover of 90.000.000 DH. Saffron cultivation is an important economic activity in the mountainous regions of Taliouine (Taroudant province) and Taznakht (Ouarzazate province). Morocco is renowned for the good quality of its saffron, which is a fagship local product and which strongly contributes to the income of local people. Improving saffron production is possible through the use of good quality plant material, but also by controlling good cultural practices and post-harvest processes. The conference will focuse on the agro-morpho-physiological stidies of saffron, it's molecular and chemical chararization and the application of tissue culture to this species. The agro-biological characterization was applied to different provenances of saffron of high, medium and low altitude and was carried out simultaneously in the original production site for each provenance, in a site in Taliouine where all the accessions were grouped and in one site with a micro-climate and altitude completely different from the original sites. Preliminary information on the agro-morphometric variability of Moroccan saffron under different planting sites, allowed us to make a first selection of accessions and planting conditions that show a clear superiority in the production of corms, leaves, flowers and qualitative performance. The simple sequence repeat markers were used to evaluate the genetic variability in different accessions of Moroccan saffron and saffron from other countries and some of them were able to trace diversity in these accessions and assign a molecular identity. The chemical study of saffron focused on the quality of spices by evaluating the content of its three main metabolites. It also aimed to control the authenticity and to distinguish different geographical origins of this spice using UV-Vis, HPLC, GC, IR and colorimetric technics. Different methods related to stigma drying, storage and preservation were compared. The auto-triploid nature of saffron creates problems for sexual reproduction and only vegetative propagation is possible. This propagation carries the risk of disease transmission and the regeneration rate of daughter corms under natural conditions is relatively low. This slow vegetative propagation constitutes significant bottlenecks for the development and improvement of saffron culture and in vitro micropropagation is used in order to produce large quantities of the propagating healthy material in saffron. This conference will outline the major advances of in vitro culture in direct and indirect organogenesis and somatic embryogenesis of saffron. 
O6. Evaluation of EMS Induced Genetic Variability in Quantitative Traits in Rapeseed (Brassica napus L.)

Souhail Channaoui ${ }^{1,2}$, Mustapha Labhilili ${ }^{3}$, Mohamed El Fechtali', Hamid Mazouz ${ }^{2}$, and Abdelghani Nabloussi $^{{ }^{*}}$

1 Research Unit of Plant Breeding and Plant Genetic Resources Conservation, National Institute of Agricultural Research, Regional Agricultural Research Center of Meknes, Morocco; ${ }^{2}$ Laboratory of Plant Biotechnology and Molecular Biology, Department of Biology, Faculty of Science, University Moulay Ismail, Morocco; ${ }^{3}$ Research Unit of Plant Biotechnology, National Institute of Agricultural Research, Regional Agricultural Research Center of Rabat, Morocco. "Corresponding and Presenting Author: abdelghani.nabloussi@gmail. com.

\section{Abstract}

Genetic variability is a prerequisite feature of any crop-breeding program and chemical mutagenesis has become a proven way of creating new variations within a crop germplasm. Seeds of Brassica napus $L$. (variety 'INRA-CZH2') were treated by Ethyl Methyl Sulphonate (EMS), with 1, 1.2, 1.4 and $1.6 \%$ doses for 6,7 and 14 hours, and were planted to obtain the M1 plants and then the M2 plants. The objective was to evaluate the novel induced variability observed for some important traits, such as days to flowering, days to maturity, plant height, number of branches/plant, number of pods/plant, number of seeds/pod, pod length, pod diameter and 1000-seed weight. Data were recorded on 10 plants taken randomly from M2 populations derived from each treatment (dose by duration), along with control plants (wild type). A large variability was observed and EMS treatment had a significant effect on all the traits studied. Compared to control plants, genotypes coming from seeds treated with low EMS doses were earlier to flowering and maturity. Also, seeds treatment by low EMS doses during short time allowed obtaining some plants with increased number of pods/plant compared to the control. Among the treatment levels used in the present research, 1\% EMS for 6 hours was found to be the most effective in improving earliness and seed yield. This latter was mainly improved due to the increase of the number of pods/plant. Keywords: Rapeseed, induced variability, EMS treatment, earliness, seed yield.

\section{Antioxidant and Anti-Cancer Activities of Anacyc- lus pyrethrum from Morocco}

Hakima Bahri ${ }^{1,2^{*}}$, Abdeslem El Idrissi ${ }^{2}$, Salvatore Rotondo ${ }^{2}$, Bouchra Tazi ${ }^{3}$, Samira Oulahboub ${ }^{1,3}$, and Chaymae Benkirane ${ }^{1,3}$

1 Laboratory of Genetic Resources and Plant Improvement, Ecole Nationale d'Agriculture de Meknès, BPS 40, Meknès, Morocco; ${ }^{2}$ Laboratory of NeuroSciences, Medical Technology Program, Department of Biology, College of Staten Island, City University of New York, New York, 10314, USA $^{3}$ Laboratory of Chemistry, Department of Basic Sciences, Ecole Nationale d'Agriculture de Meknès, BPS 40, Meknès, Morocco. ${ }^{*}$ Corresponding and Presenting Author: hbahri@enameknes.ac.ma.

\section{Abstract}

Over the ages, man has relied on plants to attend to many health problems and plants. Nowadays, a renewed interest in natural products has emerged in order to find safe and effective alternatives to the established chemical drugs. Research efforts target plants as an inexhaustible source of naturally occuring bioactive compounds that proved useful in the treatment of many human ilements, including cancer, cardiovascular diseases and inflammatory diseases. This work aims to investigate the antioxydant and anti-cancer activities of Anacyclus pyrethrum an indigenous spontaneous medicinal plant commonly prized for its roots. Pyrethrum root samples were collected from the mid-Atlas region. Plant extracts were prepared and antioxidant activity was measured by DPPH (2,2-diphenyl-1-picrylhydrazyl) free radical scavenging method and total polyphenol and total flavonoid contents using the Folin-Ciocalteu and the aluminum chloride colorimetric methods respectively. We assessed anti-cancer activity against two different cell lines, HeLa (human cervical cancer cell line) and $\mathrm{N} 2 \mathrm{a}$ (mouse neuroblastoma cell line) using the ATP assay. Cell lines were seeded in a 96 well plate at the concentration of 104 cells/well, and plant extracts were added at the concentrations 200, 150, $100,50,25$ and $12,5 \mu \mathrm{g} / \mathrm{ml}$. Cell viability was as- 
sessed by measuring ATP content by luminescence

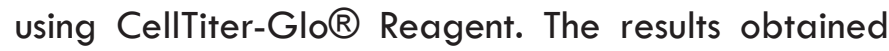
show that total polyphenol content ranged from 435 to $721 \mathrm{GAE} \mu \mathrm{g} / 100 \mathrm{mg}$ of extract and total favonoid from 166 to 721 QE $\mu \mathrm{g} / 100 \mathrm{mg}$ of extract. Pyrethrum roots exhibited an important antioxidant activity with IC50 values ranging from 3.3 to $3,8 \mu \mathrm{g} /$ $\mathrm{ml}$ compared to $3.1 \mu \mathrm{g} / \mathrm{ml}$ for ascorbic acid used as the reference antioxidant. Furthermore, the cytotoxicity assay revealed a strong anti-cancer activity of pyrethrum root extracts against the two cancer cell lines used. These findings highlight the interest of Anacyclus pyrethrum roots as an important source of polyphenols with potent antioxidant activity and with high cyto-toxicity effect against cancer. Keywords: Anacyclus pyrethrum, Polyphenol content, Flavonoid content, Antioxidant Activity, anti-cancer activity.

\section{O8. Preliminary Results on Flowering Phenology of the World Olive Germplasm Bank of Marrakech}

Zaher Hayat ${ }^{1 *}$, Moukhli Abdelmajid', Khayat Meriem', Boutzankad Tfarah',2, Gaboun Fatima', Sikaoui Lhassane $^{1}$, El Modafar $\mathrm{C}^{2}$, and Khadari Bouchaib ${ }^{3}$

'Institut National de la Recherche Agronomique, CRRA de Marrakech, BP 533 Menara, 40000 Marrakech, Morocco; ${ }^{2}$ Université Cadi Ayyad, Faculté des Sciences et Techniques, Guéliz, 40000 Marrakech, Morocco; ${ }^{3}$ INRA, UMR 1334 Amélioration Génétique et Adaptation des Plantes (AGAP), 34398 Montpellier, France. "Corresponding and Presenting Author : hayatzaher@yahoo.fr.

\section{Abstract}

A set of 591 olive accessions from the world olive collection was planted in Marrakech and characterized in 2014, 2015 and 2016 for flowering phenology according to the $\mathrm{BBCH}$ scale (the time of inflorescence buds start to swell, growth and development of inflorescences, flower opening, full flowering, full petal fall, end of flowering, fruit set). Degree days of flowering stages 51 to 69 were higher in 2015 compared to 2014 , but the highest flowering period was observed in 2016. A significant positive correlation was detected between all flowering stages except for stage 51, which is negatively correlated with flowering period estimated by the difference between the time of stage 69 and stage 61. Variance analysis showed effect of cultivar and year on all flowering stages. Stage 51 is a key stage of olive's trees phenology; its early observation is correlated with long flowering period. Flowering period observations show correlation between a short flowering period and the increase of temperature expressed by the sum of degree-days. $\left[\left(T^{\circ} \max +T^{\circ} \min \right) / 2-\right.$ $12.5^{\circ} \mathrm{C}$ ] needed to observe stage 51 . Varieties with late stage 51 need more time to satisfy their minimum chilling requirements and cumulate more degreedays before reaching the stage. In southern Mediterranean countries, we recommend selection of varieties with low chilling requirement (early stage 51 ) for better adaptation to xeric conditions in the summer period especially with the risk of global warming which is more and more considered. For future study we will focus on the estimation of the minimum chilling requirements to observe Stage 51. Keywords: Olive, Flowering, Phenology, BBCH Scale, Core, Marrakech, World, Collection. Chilling requirement, Selection.

\section{Concurrent Session II: Animal Health \& Production}

\section{Co-Chair: Karen Jones, Southern Illinois Univer- sity, USA \\ Co-Chair: Mohammed Bouslikhane, IAV, Moroc- co}

\section{O1. First Isolation and Characterization of Fowl Adeno- virus from a Field Outbreak of Inclusion Body Hepatitis Among Moroccan Broiler Chickens.}

Samira Abghour ${ }^{1 *}$, Fatima Tahiri', Khalil Zro ${ }^{2}$, Mohammed Mouahid ${ }^{3}$, Jaouad Berrada ${ }^{4}$, and Faouzi Kichou ${ }^{4}$.

1 Division of Pharmacy and Veterinary Inputs, ONSSA, Rabat, Morocco; ${ }^{2}$ Biopharma, Rabat, Morocco; ${ }^{3}$ Mouahid's Veterinary Clinic, Temara, Morocco; ${ }^{4}$ Hassan II Institute of Agronomy and Veterinary Medicine, B.P. 6206, Rabat, Morocco. "Corresponding and Presenting Author: abghour.samira@yahoo.fr. 
Inclusion body hepatitis (IBH) is an acute disease, mainly occurring in young broiler chicken (3-7 weeks old) and caused by several serotypes of fowl adenovirus (FAdV). A very few studies were conducted in Morocco based only on gross and histopathological examinations. In the present work, an outbreak of IBH in broiler chickens have been investigated through histological examination of affected birds, isolation of virus via chicken embryofibroblasts cell (CEF) and detection by conventional PCR based on hexon gene. Histopathology examination revealed large basophilic intranuclear inclusion bodies within hepatocytes. The FAdv was isolated in chicken embryo fibroblast cell culturesinoculated with tissue homogenate of livers collected from broiler chickens with IBH. The cytopathic effects detected were in the form of rounding, clumping and detachment of cells. Cell culture supernatant from second and third passages of isolateas well as the liver homogenate were subjected to $P C R$ amplification using primer pair HexFl/HexR 1 , the specific product of $800 \mathrm{bp}$ was detected in all samples tested. These results confirmed the implication of FAdv in IBH among Moroccan chicken flocks. Furthermore, in order to determine what genotype and serotype involved in this Moroccan outbreak, the FAdv isolate is now being further characterized genetically using phylogenetic analysis based on the nucleotide sequences of the PCR product and the results will be discussed and presented. Keywords: Inclusion Body Hepatitis, Fowl Adenovirus, isolation, PCR, genotype, broiler chickens.

O2. A Comparative Analysis of the Economic and Water Use Efficiencies of Camel and Other Species in the Oases' Farming Systems.

Mohamed Taher Srairi ${ }^{1 *}$, Salah Mansour ${ }^{1}$, Mohamed Benidir $^{2}$, Mohammed Bengoumi ${ }^{3}$ and Véronique Alary ${ }^{4} .{ }^{1}$ Hassan II Agronomy and Veterinary Medicine Institute, Department of Animal Production and Biotechnology, P.O. Box 6202, 10101, Madinate Al Irfane, Rabat, Morocco; ${ }^{2}$ Regional Office of Agricultural Development in Ouarzazate, Morocco; ${ }^{3}$ Sub regional office of the Food and Agriculture Organization, Tunis; ${ }^{4}$ International Center for Agricultural Research for Development (CIRAD), Montpellier, France, currently based at the International Center of Agricultural Research in Dry Areas (ICARDA), Rabat, Morocco. "Corresponding and Presenting Author: mt.srairi@iav.ac.ma.

\section{Abstract}

Multiple constraints, such as an increased demographic pressure and the competition for scarce water resources, are significantly affecting farming systems in the oases. The combination of these limiting factors impacts the efficiency of inputs' uses and hampers the incomes from the agricultural activities. Livestock has always been a component of the oasis farming systems, covering a wide range of functions: transportation, soil preservation, income generation through meat and milk, draft, and saving. Faced to the recent changes, this study aimed to characterize the roles and contribution of different livestock species (camel, cattle and small ruminants - goats and sheep -) on the overall performances of the oasis farming systems. To do so, twelve farms illustrating four types of livestock systems previously identified (i.e. three farms per type) were selected: (i) Multi species livestock rearing (sheep and cattle as well as sedentary goats of the Drâa breed), (ii) Dairy cattle based activities, (iii) Sheep intensification with an important contribution of purchased feed and (iv) Range land (camel and goats) systems. The study was undertaken in the Drâa valley (South East Morocco), a typical oasis context, with an average annual rainfall level not exceeding $110 \mathrm{~mm}$ and summer temperatures often above $45^{\circ} \mathrm{C}$. The valley consists in a series of seven palm groves along a distance of $200 \mathrm{~km}$ following the Drâa River and covering a total arable land area of 26,000 ha An annual follow-up was performed in each farm with a set of surveys coupled to field observations. Inputs' uses (water, labor, fertilizers, etc.) and incomes from crops and livestock in the study sample were determined. Off farm revenues were also characterized. Within each farm, we calculated common agricultural efficiency indicators to assess the efficiency use of the most critical production factors: land, labor, water and capital. The results demonstrated that efficient oasis farming systems rely on the crops/ livestock association. Thereby, while providing selfconsumed food products, livestock intensified farming systems (D'man prolific sheep and dairy cattle with off-farm feed resources) allow an increase in crops' yields and their incomes. This is particularly obvious 
for date palm trees, which benefit from the surplus irrigation of the underlying alfalfa. In parallel, in specific contexts of the oases where the intensification of agriculture is impossible (for instance in downstream areas, with scarce groundwater or saline water) camels remain the main source of income, adding value to the vast pastoral areas and to the by-products of crops (wastes of dates, wheat bran and straw, etc.) and inducing very limited pressure on water resources. Altogether, the results emphasize the crucial roles of camel in areas with limited assets (water, land and even capital), and suggest that such a species might be of significant interest in the near future, in case of exacerbated water scarcity, as it might happen with climate change scenarios. Keywords: camel, dairy cattle, farms' incomes, farming systems, oasis, sheep, water use efficiency.

\section{O3. Bovine Tuberculosis and Paratuberculosis Coexis- tence in Dairy Cattle in Morocco}

Meryem El Mrini ${ }^{*}$, Jaouad Berrada', Faouzi Kichou', Beatriz Romero2, Ana Balseiro Morales ${ }^{3}$, Christian Gortázar ${ }^{4}$ and Mohammed Bouslikhane'

1 Department of Veterinary Pathology and Public Health, Hassan II Institute of Agronomy and Veterinary Medicine, Rabat, Morocco; ${ }^{2}$ Centro de Vigilancia Sanitaria Veterinaria (VISAVET), Universidad Complutense, Madrid, Spain; ${ }^{3}$ Centro de Biotecnología Animal-SERIDA Gijón Asturias Spain; 4 SaBio IREC Universidad de Castilla - La Mancha, Ciudad Real, Spain. *Corresponding and Presenting Author: meryem.pathologiste@gmail.com.

\section{Abstract}

Bovine tuberculosis (TB) is a major endemic zoonosis in Morocco, with $33 \%$ of dairy cattle farms infected countrywide with mycobacteria of Mycobacterium tuberculosis complex (MTC) (FAO, 2004). According to economic and public health impacts of the disease, eradication of bovine tuberculosis is an important issue in Morocco. Public animal health autorithy recognises the in vivo single and comparative intradermal tuberculin (IDT) test as the official diagnosis assays for bovine tuberculosis field diagnosis. The in vitro interfero-gamma (IFN- $\gamma$ ) assay employment may be authorized to maximise detection of early infected cattle. Field diagnosis results interpretations are affected by the coexistence of bovine tuberculosis and other mycobacterial infections like bovine paratuberculosis (PTB) or infection by environmental mycobacteria. We aimed to investigate the coexistence of TB and PTB in dairy cattle farms in Morocco. The carcasses of 227 slaughtered IDT-positive cows originating from six dairy cattle farms were thoroughly examined and tissue samples were collected from lymph nodes without TB-characteristic macroscopic changes (retropharyngeal, mediastinal, prescapular, mesenteric and iliac lymph nodes) and from ileo-caecal valve and terminal ileon. One batch of fresh tissues was stored at $-20^{\circ} \mathrm{C}$ for isolation and identification of Mycobacteria from Mycobacterium tuberculosis Complex (MTC) by spoligotyping and PCR and Mycobacterium avium subsp paratuberculois (Map) by PCR. A second batch of bovine paratuberculosis suspected samples was fixed in 10\% of Neutral Buffered Formalin for histopathological examination and immunoperoxidase immunohisto-chemistry (IHC) confirmation. Preliminary results of isolation and identification yielded 24 MTC isolates by PCR and spoligotyping resulted in having six spoligotypes of Mycobacterium bovis (SBO120,0121,0125,0265, 0869 and 1167). Map isolation was confirmed by PCR in 3 cattle farms. Immuno-peroxidase IHC proved Map presence in paraffin embedded tissues in a fourth cattle farm. TB and PTB coexistence in cows was diagnosed in two studied dairy cattle farms, thus providing for the first time the evidence of TB and PTB coexistence among Moroccan dairy cattle.Keywords: Bovine, tuberculosis, paratuberculosis, dairy cattle.

\section{O4. Effect of the Incorporation of the Argan Press Cake Detoxified On Antioxidant Activity of Goat Milk.}

Nazha Lakram ${ }^{1,2^{*}}$, Ikram Mercha ${ }^{2}$, Rachid Kabbour ${ }^{2}$, El. Haj El Maadoudi ${ }^{2}$, Abdalah El Housni ${ }^{2}$, Mariam Naciri ${ }^{1}$.

1 Laboratory of Biodiversity, Ecology and Genome, Faculty of Sciences, University Mohammed $\mathrm{V}$ of Rabat, Avenue Ibn BatoutaB.P1014 Rabat, Morocco; ${ }^{2}$ Laboratory of Food Technology URPAF, National Institute for Agricultural Research, Rabat, Morocco. *Corresponding and Presenting Author: nazha.lakram@ gmail.com. 


\section{Abstract}

Goat milk is rich in various physiologically functional components, including proteins, vitamins (such as vitamins $E$ and $C$ ), flavonoïds, and carotenoids with antioxidant properties. Therefore, goat milk is considered to possess high antioxidant activity that resists oxidative stability and highly protects consumers from exposure to oxidative stress, which is an important characteristic of numerous acute and chronic diseases. This study aims to investigate the effect of the inclusion of the detoxified press cake from the argan tree in animal feeding on antioxidant activity of goat milk collected from Meskala region (Essaouira__Morocco). The feeding trails were conducted in the farm using 18 goats of the Alpine breed. They were divided in three lots of 6 goats each. The first lot received the detoxified argan press cake (DA), the second lot received the non-detoxified argan press cake (NDA) and the third receives the concentrate (C). The total phenol compounds content in the milk extracts was determined by spectrometry according to the Folin-Ciocalteu procedure and calculated as gallic acid equivalents (GAE). Thus, the flavonoïd content in the milk extracts was determined spectrometrically, using a method based on the formation of a complex flavonoïd-aluminum and calculated as Quercetin equivalents (QE). The antioxidant activity was evaluated through scavenging activity of the stable 1,1-diphenyl-2-picrylhydrazyl (DPPH) free radical. The results showed a significant difference $(P<0.05)$ of the antioxidant capacity of goat milk for the lot (DA) compared to the lots (NDA) and (C). Similarly, the polyphenol and favonoid content were higher in lot (DA) and (NDA) than in lot (C). Keyswords: goat milk, argan press cake, polyphenol, flavonoïd, antioxidant activity.

\section{O5. Estimation of Milk Yield Traits of Holstein Cattle Reared at Semi Arid Climate.}

\section{Abdeslem Talbi ${ }^{*}$ and Said El Madidi§.}

Laboratory of Biotechnology and Valorization of Natural Resources, Faculty of Science, BP 8 106, University Ibn Zohr, Agadir, Morocco. "Presenting Author: talbi_abdslem@yahoo.fr. \$Corresponding author: s.elmadidi@uiz.ac.ma.

\section{Abstract}

The purpose of this study is to analyze the official milk control data between 2008 and 2012 of a cattle herd consisting of 6307 Holstein cows. These cows are raised in 118 private farms of dairy cattle which are members of the agricultural cooperative COPAG. These farms are based in the irrigated perimeter of Souss-Massa exactly in southwestern of Morocco. The climate of this region is characterized by a semi-arid to arid climate with average temperatures variable between $19^{\circ} \mathrm{C}$ in winter and $27^{\circ} \mathrm{C}$ in summer and can reach $49^{\circ} \mathrm{C}$ in late spring and summer with the arrival of warm sub-Saharan winds. Rainfall is weak and irregular with an annual average of 180 and $280 \mathrm{~mm}$ per year. The Production parameters were analyzed using a general linear model (GLM) including fixed effects: calving season, calving year, lactation number and calving age. The averages of production recorded by this Holstein herd are 6578.30 \pm 1619.06 $\mathrm{kg}$ for the 305 days-milk yield, $7034.88 \pm 1842 \mathrm{~kg}$ for total milk yield, $3.70 \pm 0.53 \%$ for standard fat content (FC) and $325.10 \pm 42.96$ days for lactation length. The analysis of variance of production parameters depending on non-genetic factors demonstrated very highly significant effects of the calving year and calving season $(P<0.001)$ for all the parameters of production studied, with high production during the cold seasons (Autumn and winter) comparing to hot seasons (spring and summer). The lactation number has a very highly significant influence $(P<0.001)$ for all parameters except the fat content whose effect id non-significant $(p>0.05)$. Whereas, the effect of calving age was non-significant $(p>0.05)$ for these parameters of production. The production of this herd has shown a great variation between animals, which reveals the heterogeneity of this Holstein herd. The great sensitivity of cows to seasonal and interannual variations shows the important effect of heat stress caused by high temperatures in hot seasons. Keywords: Holstein cattle, milk yield, non-genetic factors, semi arid climate.

\section{O6. Prevalence of Campylobacter spp. in Industry Broil- ers from the Area of Marrakech-Safi, Morocco.}

Raja Asmai $i^{{ }^{*}}$, Reda Triqui', Hakim Karib', Khadija Essoucratti ${ }^{2}$, Brahim Bouchrif ${ }^{3}$, and Rachid Gradess ${ }^{3}$. 
Department of Pathology and Veterinary Public Health, Agronomic and Veterinary Institute Hassan II, Rabat; ${ }^{2}$ Department of Biology, Ain Chock Faculty of Sciences, Hassan II University, Casablanca; 3Food Microbiology Laboratory Pasteur Institute of Morocco, Casablanca, Morocco. *Corresponding and Presenting Author: rajaevet@gmail.com.

\section{Abstract}

In Morocco, the incidence of human campylobacteriosis has not been evaluated so far. Prevalence studies are a prerequisite for any risk assessment studies. The current investigation aimed at studying the prevalence of Campylobacter spp. in farm broilers in the Marrakech-Safi area of Morocco. This was carried out in the period of July-October 2017. 90 samples of cloacal swabs were collected from broilers in the 8 provinces of the study area. This represented 30 farms with an average of 3 broilers per farm. Isolation and identification of Campylobacter spp. were performed according to a Moroccan standard methods and recommendations of the OIE and WHO (NM O8.O.135/ Standard NM ISO/TS 10272-3, 2013). Thermotolerant Campylobacter species (C. jejuni and C .coli) were isolated in $73,34 \%$ of the farms investigated. Our results revealed the presence and the probable spread of these two species in the broiler farms within the 8 provinces of the study area. Such results are in agreement of an increasing risk of broiler carcass contamination at the slaughter level, along with the possible hazard transfer to consumers under circumstances of either undercooking or cross-contamination at the preparation level. Keywords: Thermotolerant Campylobacter, cloacal swabs, Prevalence; Broiler industry.

O7. Epidemiological Investigations of Small Ruminant Abortions in the Regions of Khemisset and El Hajeb.

Faouzi Kichou* ${ }^{*}$, Fatima Ezzahra Belhassan, Mohammed Bouslikhane, Ahlam Kadiri, and Jaouad Berrada.

Département de Pathologie et Santé Publique Vétérinaires, IAV Hassan II, Rabat, Morocco. "Corresponding and Presenting Author: f.kichou@iav.ac.ma.

\section{Abstract}

Abortions in small ruminants deserve particular attention because of their heary economical burden. The main objective of this study was to evaluate the impact of abortions in small ruminants in two regions of Morocco (Khémisset and El Hajeb regions) and to determine the seroprevalence of main abortive infections (Brucellosis, Chlamydophilosis, Q fever, Toxoplasmosis and Neosporosis) and their involvement in some abortion outbreaks. Sero-epidemiological survey was carried out in 57 flocks (36 sheep flocks, 14 herds of goats and 7 mixed herds) originating from 4 rural counties (Had Lghoualem, Ait lchou, Ait Bourzouine and Ait Hraz Allah). Data about herd structure, reproductive performances and abortion events were collected. In parallel, A total of 433 blood samples were taken from 329 sheep and 104 goats with or without history of abortion. Harvested sera were examined for Brucellosis using Rose Bengal Test (TRB) and then subjected to ELISA in order to search for Chlamydophilosis, Q Fever, Toxoplasmosis, and Neosporosis. Results revealed abortion rates ranging from $1.5-10 \%$ in sheep and from $0.8-7.3 \%$ in goats in the county of Had-Laghoualem, from $8-11.2 \%$ in sheep and from $5.8 \%-44.4 \%$ in goats in the county of Ait Ichou, from $0.8 \%-10 \%$ in sheep and from $5-8 \%$ in goats in Ait-Bourzouine, and abortion rates ranging from $0.5-5 \%$ in sheep in the county of Ait-HrazAllah. Among the examined 57 flocks/herds, $47.3 \%$ were positive for toxoplasmosis with a seropositivity rates (SR) of $21.2 \%$ in sheep and $2 \%$ in goats, $40.3 \%$ tested positive for Chlamydophilosis with a SR of $9.4 \%$ in sheep and $36.5 \%$ in goats, $45.6 \%$ were positive for $Q$ fever with an SR of $10 \%$ in sheep and $8.6 \%$ in goats, and $21 \%$ were tested positive to neosporosis with a SR of $4 \%$ in sheep. No flock was found to be serologically brucellosis positive. These results reflect the relative importance and implication of these abortive infections in the occurrence of abortions and the need for confirmation by direct examinations. Furthermore, the present work documents for the first time in Morocco the serological evidence of infection with Neospora caninum in sheep, which deserves further investigations. Keywords: Abortion, small ruminants, soats, sheep, Khémisset, El Hajeb, brucellosis, chlamydophilosis, $Q$ fever, toxoplasmosis, neosporosis, serology. 
O8. Spring and Autumnal Hormonal Variations of Ouled Diellal Ewes in Algeria

\section{Azdinia Zidane $^{1^{*}}$ and Sarra Metlef ${ }^{2}$}

${ }^{1}$ Laboratory of Natural Bio-Resources, Department of Biology, Faculty of Nature and Life Sciences, Hassiba Benbouali University of Chlef, PO Box 151, (02000) Chlef, Algeria; ${ }^{2}$ Laboratory of Natural Bio-Resources, Department of Nutrition and Food sciences, Faculty of Nature and Life Sciences, Hassiba Benbouali University of Chlef, PO Box 151, 02000 Chlef, Algeria. "Corresponding and Presenting Author: azdinia. zidane@yahoo.fr.

\section{Abstract}

The photoperiodism represents the capacity of plants and animals to measure the duration of day; this process implies certain biological calender which allows the bodies to determin the convenient moment of the year to make a commitment in appropriate seasonal adaptations. During the annual cycle, animals are often exposed to a range of gradually increasing and decreasing photoperiods; these seasonal annual variations of photoperiod are at the origin of numerous biological and behavioral changes in animals. So, it is admitted that the photoperiodism is the main factor controlling the seasonal variation of the sexual activity of ewes. However, the external manifestations of heat in ewes are difficult to observe, as they are very discreet. So, the use of indirect methods of heat detection or the control of cycles is therefore justified in ovine species. The purpose of this study was to check if the Ouled Diellal ewes followed a seasonal or an unseasonal profile in the region of Chlef, and to study the hormonal profiles: pituitary hormones (FSH and $\mathrm{LH}$ ) and gonadal hormones (progesterone and œstradiol). The study involved 60 ewes of Ouled Diellal breed, adults, healthy and non-pregnant. The blood samples were carried out from the jugular vein, 2 times a week in spring (April to June) and in autumn (October to December). The hormones have been assayed using the ELISA technique. The results did not reveal any significant difference $(P<0.05)$ between hormone concentrations during both seasons. It is so showed that the ovarian follicular activity of the Ouled Diellal ewes in the region of Chlef is also present during the seasonal light anoestrus; this leads us to confirm the unseasonal profile of this breed. Keywords: Autumn, hormonal assay, FSH, LH, œstradiol, progesterone, spring.

\section{Concurrent Session III: Bioactive Com- pounds \& Genetic Diversity}

\author{
Co-Chair: El Hassan Achbani, INRA, Morocco \\ Co-Chair: Karen Midden, Southern Illinois Uni- \\ versity, USA
}

O1. Environmentally and Genotypic Induced Shifts in Reproductive Phenology of Some Almond [Prunus dulcis (Mill.) D.A. Webb] Cultivars Grown in North-Eastern Morocco

El Hassan Sakar ${ }^{*}$, Mohamed El Yamani, Abdeali Boussakouran, and Yahia Rharrabti

Laboratory of Natural Resources and Environment, Polydisciplinary Faculty of Taza, Unviersity Sidi Mohamed Ben Abdellah, B.P 1223, Taza, Morocco. *Corresponding and Presenting Author: hassan.sce@ gmail.com/elhassan.sakar@usmba.ac.ma.

\section{Abstract}

In Morocco, almond [Prunus dulcis (Mill.) D.A. Webb] is the most important nut crop among the rosaceous family both in terms of cultivated acreage and production. The modern Moroccan orchards are characterized by the dominance of some commercial cultivars such as Marcona, Fournat de Brézenaud, Ferragnès, Ferraduel, and Tuono which were released by European almond breeding programs. To optimize agronomic practices in almond orchards (pollination, pruning, harvesting ...), key phenological events must be known. Unfortunately, an accurate knowledge of their phenology is far to be investigated before, hence the novelty of our work. We investigated some key reproductive phenological events of almond from the five widely almond cultivars: Marcona (MAR), Fournat de Brézenaud (FBZ), Ferragnès (FRG), Ferraduel (FRD), and Tuono (TUO). This study was undertaken over two crop seasons (2016 and 2017) across five different sites from north-eastern Morocco namely: Aknoul 
$(A K)$, Bni Hadifa (BH), Rislane (R), Sidi Bouhria (SB), and Tahar Souk (TS). These cultivars were marked in triplicate. Since 2015 season, these were visited periodically and their reproductive phenology was assessed according to the general $\mathrm{BBCH}$ scale and reported as Julian days. Phenological events investigated consisted were as follow: initial blooming date (BBCH61), full blooming date (BBCH65), final blooming date ( $\mathrm{BBCH} 69)$, blooming duration, and ripening date (BBCH89). ANOVA analyses demonstrated significant effects of cultivar, site, crop season, and their interactions on the whole studied phenological events. LSD's test demonstrated wide variabilities between cultivars as regards to their reproductive phenology. Besides, important shifts were detected among the five localities, but also between the two crop seasons for flowering and ripening times. The north's sites ( $\mathrm{AK}, \mathrm{BH}$, and TS) showed an extended blooming duration as compared to the eastern's sites (SB and $\mathrm{R})$. Blooming time in our cultivars ranged from middle (FBZ and MAR) to later (FRD, FRG, and TUO). Our results revealed also, a strong negative correlation was found between initial blooming date and blooming duration, while ripening time was independent from blooming time. Shifts in ripening time between cultivars are highly desirable trait when several cultivars are grown simultaneously in the same orchard. Keywords: Reproductive Phenology, Commercial Almond, North-Eastern Morocco.

O2. Gas Chromatography-Mass Spectrometry Profile of Essential Oil from Asteriscus graveolens (Forssk) and its Antifungal Activity Against Fusarium oxysporum $f$. sp. albedinis, the Causal Agent of "Bayoud" Disease on Date Palm.

El Mustapha Chibane ${ }^{1^{*}}$, Adil Essarioui ${ }^{2}$, Mohamed Ouknin', Amal Boumezzourh', and Lhou Majidi'. ' Laboratory of Natural Substances \& Molecular Synthesis and Dynamics. FST, Errachidia, Morocco; ${ }^{2}$ National Institute for Agricultural Research Regional Center, Errachidia, Morocco. . "Corresponding and Presenting Author: elmustaphachibane@gmail.com.

\section{Abstract}

Date palm (Phoenix dactylifera) is an important food source and commercial perennial crop in the Sahara and North Africa. "Bayoud", a vascular wilt disease, incited by the soilborne fungal pathogen Fusarium oxyxporum f. sp. albedinis (Foa), has become a serious threat to date production in date palm-growing regions in Morocco. Substantial body of research reported antifungal properties of a wide range of plant extracts. The present work aims to determine chemical composition and anti-Foa activity of essential oil extracted from the aromatic and medicinal plant Asteriscus graveolens (Forssk). Gas chromatography-mass spectrometry analysis revealed the presence of twenty-five distinct chemical components in the essential oil samples. Mycelial growth and spore germination of Foa were found to be strongly inhibited by the oil when tested using agar dilution assay. The minimum inhibitory concentration of the oil was $0.6 \%(\mathrm{v} / \mathrm{v})$, and its fungicidal effect was reached at a concentration of $13.33 \%(\mathrm{v} / \mathrm{v})$. These findings contribute significant insight into the potential of using plant extracts for controlling "Bayoud" disease. Keywords: "Bayoud" disease, Asteriscus graveolens (Forssk), Fusarium oxysporum f. sp. albedenis, antifungal activity

\section{O3. Antioxidant Activity of Camel Milk: Effect of Incor- poration of Argania spinosa's Sub Products.}

Ikram Merch ${ }^{1,2^{*}}$, Nazha Lakram ${ }^{2}$, Med Rachid Kabbour ${ }^{2}$, Aouatef Benali ${ }^{2}$, Fouzia Zkhiri ${ }^{1}$, and El Haj El Maadoudi².

1 Laboratory of Virology, Microbiology \& Quality / Eco-toxicology \& Biodiversity University Hassan II, Faculty of Science and Techniques of Mohammedia, PB 146 Mohammedia 20650 Morocco; ${ }^{2}$ The National Institute of Agronomic Research, RCAR-Rabat, PB 6570, Institutes Rabat, 10101 , Rabat Morocco. ${ }^{*}$ Corresponding and Presenting Author: ikram.mercha@ gmail.com.

\section{Abstract}

Phenolic compounds are increasingly of interest in the food industry because they retard oxidative degradation of lipids and thereby improve the quality and nutritional value of food. Many studies reported that beneficial bio molecules compounds have been identified from various parts of the Argania spinosa's (L.) Skeels and can play an important role in fighting diseases and could be used in pharmaceutical and 
personal care products. In this present study we investigate the effect of the incorporation of the argan tree's sub product in animal feeding, on the antioxidant activity of camel milk collected from Essaouira region and compare it to the milk of a control population argan free. The content of total phenolics in the extracts was determined spectrometrically according to the Folin-Ciocalteu procedure and calculated as gallic acid equivalents (GAE). The antioxidant properties were evaluated by the scavenging test of the diphenyl picryl hydrazyl (DPPH) radical. The results obtained show that the polyphenol content of camel milk extracts following a diet based on the argan sub products is higher compared to that of the control group, also a greater capacity to trap the DPPH radical with an average of $82.85 \%$. Keywords: Camel milk, Argania spinosa, polyphenolic compounds, antioxidant activity.

\section{O4. Preservation of Aromatic and Medicinal Plant Re- sources: Case of Thymus Spp.}

\section{Zineb Neijar El Ansari ${ }^{*}$ and Ahmed Lamarti}

Laboratory of Plant biotechnology, Department of Biology, Faculty of Sciences, Abdelmalek Essaadi University, M'hannech II, B.P.21 21, Tetouan, Morocco. ${ }^{*}$ Corresponding and Presenting Author: zinebnejiarelansari@hotmail.com.

\section{Abstract}

The extension of Thymus Spp. culture, as an endemic species in Morocco and as producers of essential oil rich in bioactive molecules (thymol, p-cymene, $\gamma$-terpinene, $\beta$-caryophyllene, carvacrol...), requires to make a very large number of plants available to farmers in a very short time. These plants must be able to give a high yield of essential oil and be morphologically and phonologically homogeneous, to facilitate cultural operations. If the high oil content can be obtained by varietal selection, the obtaining of homogeneous material passes through in vitro propagation. This latter comprises a number of techniques divided into three categories, namely micropropagation by apex culture, micropropagation by adventitious budding and somatic embryogenesis. To find a large-scale application, propagation methods must be reliable, simple and reproducible. For now, only micropropagation from axillary buds seems to be the technique to develop, if we can reduce culture cycle and significantly increase the rate of multiplication to make production profitable. Thus, we established an in vitro vegetative multiplication protocol from stem nodes of three Thymus species (Thymus broussonetii Bois., Thymus pallidus Coss., Thymus vulgaris L.). As a result, we found that mineral and hormonal compositions, as well as the succession of different culture media influenced the multiplication and development of explants. Indeed, MS (Murashige and Skoog) and N30K (Margara) macronutrients give the best results. In addition, the type of cytokinin present in the medium plays a determining role in the proliferation of apex. Also, the presence of polyamines seems to be favorable for in vitro propagation. On the other hand, the acclimatization of rooted explants of 12 16 weeks has been successfully established. Thereby, this study suggests that the production of Thymus vitro-plants still needs to be improved, by modernizing protocols that provide producers in the sector with plant material that is homogeneous, adapted to the growing conditions and performing well in terms of yields and costs. Keywords: Preservation, Aromatic and Medicinal Plants, Thymus Spp., Apex Culture, In vitro Propagation.

\section{O5. Chemical Composition, Antioxidant, and Antibacte- rial Acitivity of Argania spinosa Pulp.}

Fatima Zahrae Zouhair $\frac{12^{*}}{}$, Loubna Rachidi ${ }^{2}$, Younes Ennahli ${ }^{2}$, Aouatif Benali ${ }^{2}$, Mohamed Rachid Kabbour ${ }^{2}$, Mohamed Bouksaim², and Azzouz Essamri'.

1 Laboratory of Agroresources and Process Engineering, Faculty of Sciences, University Ibn Tofail, B, P1 4000 Kenitra, Morocco; ${ }^{2}$ Laboratory of Food Technology URPAF, National Institute for Agricultural Research, Rabat, Morocco. *Corresponding and Presenting Author: fatidoc.89@gmail.com.

\section{Abstract}

Argan (Argania spinosa (L) Skeels), endemic and emblematic tree to Southwestern Morocco, where it represente the lonely species of the tropical of Sapotaceae, occupies an area of around 820,000 ha (Charrouf et al., 2002). It is the most remarkable in North Africa, due to its economic, botanical and bio- 
ecological interest as well as its social value. The yield of fresh fruit varies according to tree, environment and climate season. It is $500 \mathrm{~kg} / \mathrm{ha} /$ year per hectare on average (M'Hirit, 1989) and about 15 $\mathrm{kg} /$ tree (Rahali, 1989). Recently, many beneficial bio-molecules compounds have been identified from various parts of the A. spinosa, which can play a beneficial role in fighting disease and could be used in pharmaceutical and personal care product industries. The puprose of this work is to optimize the parameters extraction of bioactive molecules from the pulp (pericarp) and oil cake part of Argania spinosa (L.) Skeels (A. spinosa) of Tiznit region. The first step was devoted to study the effect of solvent polarity and extraction technique on the phytochemical screening and quantitative caracterisation of the extracted compounds. The second step was designed to the investigation of the antioxidant capacity and the antibacterial activity of the extracts containing more phenolic compounds. Keywords: Argania spinosa pulp, Optimization, Extraction, Bioactive molecules, Antioxidant, antibacterial activity, phenolic compounds.

\section{O6. Validation of Antifungal Activity of Essential Oils from Competitive-Ruderal Plants}

Khadiia Basaid ${ }^{1 *}$, B. Chebli ${ }^{1}$, J.N. Furze ${ }^{2}$, E.H. Mayad ${ }^{2}$, and R. Bouharroud ${ }^{3}$

' Biotechnology and Environmental Engineering Team, Laboratory for Process Environmental and Energy Engineering, National School of Applied Sciences, Ibn Zohr University, PO Box: 1136/S, Agadir, Morocco; ${ }^{2}$ Laboratory of Biotechnologies and Valorization of Natural Resources, Faculty of Sciences - Agadir, Ibn Zohr University, Agadir, Morocco; ${ }^{3}$ Integrated Crop Production Unit, Institut National de la Recherche Agronomique, Agadir, Morocco. ${ }^{*}$ Corresponding and Presenting Author: khadija.basaid@gmail.com.

\section{Abstract}

Senecio glaucus ssp. Coronopifolius (Maire) and Ridolfia segetum (L.) are competitive-ruderal plants growing in Morocco. Their essential oils were obtained by hydro-distillation, using a Clevenger-type apparatus, and tested for antifungal activity in vitro against Botrytis cinerea. S. glaucus and $R$. segetum produced $68 \%$ and $80 \%$ inhibition of mycelial growth at 640 $\mu \mathrm{l}$ in the volatile phase, and $83 \%$ and $98 \%$ at $16 \mu \mathrm{l} /$ $\mathrm{ml}$ by the poisoned food method respectively. Essential oils evaluated in this study are antifungal agents against $B$. cinerea, and can therefore be considered as a natural substitute for chemical pesticides. This study constitutes the first step in calculation of inhibition of the fungus by the oils. Qualification and quantification of essential oils will specify measurable units, creating prediction of inhibition. Keywords: Senecio glaucus, Ridolfia segetum, competitive-ruderal, essential oils, antifungal, Botrytis cinerea.

\section{O7. Evaluation of antioxidant and phytotoxic activity of extracts of flower buds of Capparis spinosa}

Fatima-Zahra Ennacerie ${ }^{1 *}$, Fouzia Rhazi Filali ${ }^{1}$, Najia Moukrad', Amar Bentayeb2, and Mohammed Bouidra $^{2}$

1 Department of Biology, Team Microbiology and Health, Laboratory Chemistry Biology Applied to the Environment, Faculty of Science, University Moulay Ismail, Meknes, Morocco; ${ }^{2}$ Department of Chemistry, Team Physical chemistry condensed matter, Faculty of Science, University Moulay Ismail, Meknes, Morocco. "Corresponding and Presenting Author: amsam.ennacerie@gmail.com.

\section{Abstract}

The purpose of this study is to valorize the spontaneous Capparis spinosa plant harvested from western Morocco and to rationalize its use by evaluating its antioxidant activity and to test the degree of its toxicity on plant eukaryotic cells. The antioxidant activity of aqueous and ethanolic extracts of flower buds was evaluated by two methods FRAP and DPPH. The cytotoxicity of the extracts was evaluated by the seed germination inhibition test of Lepidium sativum. The different extracts have an antioxidant activity that is similar to that of vitamin $C$ for the aqueous extract of flower buds. The test of toxicity of the aqueous extract of this organ is revealed by anti-germinating effect of moderate intensity, which is reversible after rehydration of Lepedium sativum seeds. His antimitotic activity is expressed by inhibition of elongation and growth seedlings. Capparis spinosa is a plant of nutritional qualities particular for its high antioxidant effect, and its tolerance by eukaryotic cells vegetari- 
an. Keywords: Capparis spinosa, flower buds, ethanol extract, 10\% decoction, antioxidant activity, antimitotic effect, germination, Lipidium sativum seeds.

\section{O8. Multivariate Screening of Locals and Foreign Figs (Ficus Carica L.) in Northern Morocco}

Lahcen Hssaini $\frac{12^{*}}{}$, Hafida Haninie ${ }^{2}$, Rachid Razouk', Ibtissame Guirrou ${ }^{1,3}$, Assia Ejiilani ${ }^{1,2}$, Abderrahman Mekaoui ${ }^{1}$, and Jamal Charafi ${ }^{1 \S}$

1 National Institut of Agricultural Research (INRA), regional center of Meknes, Morocco; ${ }^{2}$ Laboratory of Bioprocess and Bio-Interfaces, Faculty of Science and Technology, Beni Mellal, Morocco ; 3 Laboratory of food technologies, Qualipole, Meknes, Morocco. *Presenting Author: hssaiini@gmail.com. \$Corresponding Author: jcharafi@gmail.com.

\section{Abstract}

This work aims to assess pomological and biochemical characteristics of fig fruit of 140 known local and foreign genotypes belonging to INRA Meknes collection in Ain Taoujdate. Multivariate analysis was made on the basis of 43 pomological and biochemical characters on samples of 15 fruits for each genotype. Results showed a very high significant variability in pomological traits between genotypes except for ostiole width. Fruit weight varied form 12.39 $\pm 0,9$ $\mathrm{g}$ to $87.03 \pm 2,8 \mathrm{~g}$ with an average $36,04 \pm 1,84 \mathrm{~g}$. Also, a very highly significant variability was revealed among samples based on their colorimetric parameters. More than $65 \%$ of genotypes have a very high lightness index ( $\left.L^{*}\right)$ values that varied between 46 and 83.6. The variables chart has shown some strong correlations varied from $r^{2}=0.4(p<0.05)$ to $r^{2}=0.86$ $(p<0.01)$ distinguishing neck and ostiole dimensions from the fruit form descriptors. However, the total soluble solids was less discriminant. The agglomerative hierarchical clustering based on Pearson correlation has revealed tree distinct and homogeneous groups. Particularly, the genotype "Grosse dama blanche 2259" has been largely distinguished from the others. The qualitative characterization has highlighted an important variability among genotypes regarding their morphological quality. The results showed a dominance of globose $(67 \%)$ and ovoid $(70 \%)$ shapes of figs. About $82 \%$ of genotype present ribs and over than $72 \%$ present skin cracks. The Multiple Correspondence Analysis (CMA) has revealed tree groups especially differentiated by shape, color, juiciness and pulp characteristics. Biochemical analysis has also revealed a very high significant variability among samples in total soluble sugars, total phenols and total flavonoids contents. The values recorded, attest of a high nutritional potential of the evaluated genotypes. Keywords: Ficus Carica L., Pomology, biochemical analysis, characterization, multivariate analysis.

\section{Characterization and phylogenetic diversity of Al- lorhizobium vitis isolated from grapevine in Morocco}

Khaoula Habbadi ${ }^{1,2,3^{*}}$, Quentin Duplay ${ }^{3 *}$, David Chapulliot $^{3}$, Isabelle Kerzaon ${ }^{3}$, Rachid Benkirane ${ }^{2}$, Abdellatif Benbouazza', Florence Wisniewski-Dyé ${ }^{3}$, Céline Lavire $^{3}$, Ludovic Vial ${ }^{3}$, and El Hassan Achbani ${ }^{1}$

' Laboratoire de recherche et de protection des plantes URPP-INRA-Meknès, Morocco; ${ }^{2}$ Laboratoire de Botanique, Biotechnologie, et Protection des Plantes, Faculté des Sciences, Kenitra, Morocco; ${ }^{3}$ Université de Lyon, Université Claude Bernard Lyon 1, CNRS, UMR 5557, Ecologie Microbienne, INRA, UMR 1418 , 10 Villeurbanne, F-69622, France. "Corresponding and Presenting Author: khaoula405@gmail.com.

\section{Abstract}

Crown gall was observed in recently planted vineyards of the Meknes region (Morocco). 82 isolates from 10 affected vineyards were characterized by recA sequencing and were found to belong to A. tumefaciens genomospecies G1, G4 or G7, Rhizobium rhizogenes, and to Allorhizobium vitis. Only the $39 \mathrm{~A}$. vitis isolates showed to be pathogenic in vitro on tomato and MLSA phylogenetic analyses revealed a poor genetic diversity, with the definition of only four genomic groups. Definition of the A. vitis genomic groups correlates with specific pathogenic traits: indeed, genomic groups differ with respect to the severity of hypersensitive response symptoms on tobacco leaves, the intensity of necrotic response on grapevine explants, and opine profiles. Both vitopine and octopine were detected by UHPLC in tumors induced by isolates of three genomic groups, an opine signature scarcely ever reported. Keywords: Crown 
gall, grapevine, Allorhizobium vitis, Agrobacterium tumefaciens, phylogenetic diversity.

\section{Concurrent Session IV: Water \& Soil Management}

Co-Chair: Clarice Coyne, USDA-ARS, Pullman, WA, USA

\section{Co-Chair: Ahmed Elamrani, University Mohamed I, Morocco}

O1. Enhancing Faba Bean (Vicia faba L.) Germplasm for Increased Resilience to Temperature Extremes and Improved Nutrition Quality.

Jinguo $\mathrm{Hu}^{1}{ }^{2}$, Fouad Maalouf ${ }^{2}, \mathrm{Yu} \mathrm{Ma}^{3}$, Erik Landry', Haixiao Dong ${ }^{3}$, Charles Hawkins ${ }^{3}$, Zhiwu Zhang ${ }^{3}$, Clarice Coyne ${ }^{1}$, Long-Xi Yu ${ }^{1}$, Aladdin Hamwieh ${ }^{4}$, and Shiv Kumar ${ }^{5}$.

1 USDA, Agricultural Research Service, Western Regional Plant Introduction Station, Washington State University, Pullman, WA, USA; ${ }^{2}$ International Centre for Agricultural Research in Dry Areas (ICARDA), Terbol-Lebanon; ${ }^{3}$ Department of Crop and Soil Sciences, Washington State University, Pullman, WA, USA; ${ }^{4}$ International Center for Agricultural Research in Dry Areas (ICARDA), Cairo Egypt; 5International Center for Agricultural Research in Dry Areas (ICARDA), Rabat-Morocco. "Presenting and Presenting Author: jinguo.hu@ars.usda.gov.

\section{Abstract}

Our research on the enhancement of faba bean ( $\mathrm{Vi}$ cia faba L.) has the overall objectives of increasing resilience to temperature extremes and improving nutritional quality. We conduct traditional field/greenhouse experiments and adopt contemporary genomics approaches in our independent and collaborative research projects. We released four winter-hardy germplasm lines for cover and pulse crop development and we quantified the low molecular weight carbohydrates in mature and immature seeds of 40 faba bean genotypes. More recently, in collaboration with ICARDA, Terbol, Lebanon and supported partial- ly by a grant from the CGIAR-U.S. University Linkages Program, we collected data for eight agronomic traits on an ICARDA faba bean reference set of 140 accessions grown under high temperature at different locations in Lebanon and USA. We genotyped the reference set with the genotyping by sequencing (GBS) technique which produced approximately 4 million reads per genotype on an Illumina HiSeq $2500 \mathrm{Se}-$ quencer. The single nucleotide polymorphism (SNP) marker calling program UNEAK (Universal Network Enabled Analysis Kit) found 10,950 variant loci from the sequence data. After fileting SNPs with missing rate over $60 \%$ and minor allele frequency less than $5 \%, 2,632$ SNPs were obtained for final analyses. The final dataset contained 131 accessions with SNP call rate above $70 \%$. The missing SNPs were imputed by Linklmpute. Genome-Wide Association Study (GWAS) was performed using the program BLINK (Bayesian-information and Linkage-disequilibrium Iteratively Nested Keyway). Four SNP markers were identified to be significantly associated with plant height (2 SNPs) and seed size (2SNPs). We look for new opportunities in collaborative research on faba bean germplasm, genetics and genomics. Keywords: faba bean, low molecular weight carbohydrates, heat tolerance, cold.

\section{O2. Agronomic Performance of Commonvetch (Vicia} sativa L.) and Forage Pea (Pisum sativum L.) in Pure Culture and Mixed Withbarley in the Souss-Massa Region and their Effect on Soil Fertility.

Mimouni Abdelaziz*, Wifaya Ahmed, Bouharrou Rachid, Moukrini Fouad, and Karra Youssef.

National Institute for Agricultural Research, CRRA, Agadir, Morocco. * Corresponding and Presenting Author: mimouniabdelaziz@yahoo.fr.

\section{Abstract}

In the Souss-Massa region, the rotations adopted are generally characterized by a dominance of cereals, fodder (maize for silage and alfalfa) and vegetables. Also, in some area the rotations practiced are based on barley, corn and saffron with a lack of rotations based on legumes. The introduction of forage legumes in the region is an alternative for a diversification of livestock feed. Trials in different lo- 
calities have shown that vetch and pea fix significant amounts of atmospheric nitrogen under the edaphic and climatic conditions of the Souss region. The vetch yield average was $132 \mathrm{~kg} / \mathrm{ha}$ and $126 \mathrm{~kg} / \mathrm{ha}$ in mixed crops and peas yields were 114 and $93 \mathrm{~kg} /$ ha, respectively. Howeve, the contribution of symbiotic fixation in the accumulation of nitrogen by vetch was $61 \%$ and $82 \%$ in mixture. This contribution for peas was $76 \%$ and $94 \%$ in mixed crops. Mixed crops also have the advantage of reducing disease attacks, pests and weeds. These two legumes have a symbiotic nitrogen binding capacity negatively affected by lack and excess of water, excess nitrogen and deficiency of phosphorus and potassium. Trials results also showed that barley-corn and maize-maize rotations decreased soil organic matter by $22-31 \%$. On the other hand, rotations based on both legumes significantly improved soil organic matter. The barley-corn and maize-maize rotations have the same behavior and induced an increase in the apparent density of 4 and $8 \%$, respectively. However, legume cultivated alone or in mixed crops reduced apparent soil density by $10 \%$. Barley-corn and maize-maize rotations induced respectively a severe decrease in soil water retention between 29 and 36\% compared to the initial value. Conversely, the two legumes cultivated alone and in mixed crops could significantly improve soil water retention between 24 and $26 \%$. Rotations of vetch and pea cultivated alone and mixed crops significantly enriched soil with microorganisms relative to barley-corn and maize-corn rotations. Keywords: Intensification, vetch, pea, symbiotic fixation, soil fertility.

\section{O3. Irrigation Management Improvement of Green- house Tomato by Transpiration Models in the Souss- Massa Region.}

Ahmed Wifaya ${ }^{1 *}$, Mohamed Boutfirass ${ }^{2}$, Rachid Bouharroud', Khalid Azim', Fouad Elame', Lahcen Bouirden $^{3}$, Lahoucine Gourdo ${ }^{3}$, Khalid Lekouch ${ }^{3}$, Mohamed El Jazouli ${ }^{3}$, Youssef Karra', Fouad Mokrini', and Abdelaziz Mimouni'.

1 Regional Center for Agricultural Research Agadir, Morocco; ${ }^{2}$ Regional Centre for Agricultural Research Settat, Morocco; ${ }^{3}$ Thermodynamics and Energetics Lab., Faculty of Sciences Agadir, Morocco. "Corresponding and Presenting Author: wifaya_ahmed@ yahoo.fr.

\section{Abstract}

This study compares the performance of four models to estimate the daily evapotranspiration (ET) under greenhouse. The first, De Villele model (ETDV), Penman-Monteith model (ETPM), Stanghellini model (ETST) and finally the transpiration model based on the heat balance equation at the cover, Takakura (ETTK). The work was conducted during two tomato production seasons 2012 and 2013 under two greenhouses types; Monospan and Canarian, located at Experimental Station of Melk Zhar in Balfae (INRA Agadir). ST models and PM correctly simulate tomato transpiration for both measured values for daily gain of the tomato stem (MC/DG) Monospan greenhouse ( $r^{2}=0.55$ and 0.62). Similarly, we have reached a satisfactory relationship between the Eff-TR (the efficiency of plant transpiration) and the report MC / DG using the ST model under Monospan and Canarian greenhouses $\left(r^{2}=0.67 ; 0.58\right)$ respectively. Consequently, we identified critical thresholds or baselines water status of tomato; water comfort $(0.40<M C /$ DG $<0.50$ and $70<$ Eff-TR $<90 \%$ ); overestimation of irrigation (MC / DG <0.42 and Eff-TR $<70 \%$ and water stress (MC/DG > Eff-TR 0.46 and $>90 \%$ ). In addition, the comparing means depending on the yield level has identified three groups of ET models ; STa (318T / ha) > TKb (272t/ha) > DVc PMc (236249t / ha). Keywords: Tomato, greenhouse, irrigation management, evapotranspiration models.

O4. A Comparative Study of the Effect of 2,4-Dichlorophenoxyacetic Acid Hormonal Pretreatment and Cold Pre-treatment on the Gynogenetic Response of Five Moroccan Barley Varieties (Hordeum vulgare L.).

Rabouan Oubaidou $^{1 *}$, S. Hentour ${ }^{1}$, Y. El Goumi ${ }^{1}$, S. Essayagh $^{2}$, O. Lamsaouri', and M. Fakiri'. ' Agri-food and Health Lab, Hassan I University, Faculty of Science and Technology, B.P. 577, Settat, Morocco; ${ }^{2}$ Biochemistry and Neuroscience Lab, Hassan I University, Faculty of Science and Technology, B.P. 577, Settat, Morocco. *Corresponding and Presenting Author: rabouanoubaidou@gmail.com. 


\section{Abstract}

Haplomethods are key during the process of varietal creation. We aimed to improve the protocols of the haplomethods used in our laboratory to enhance the induction rate in calluses and/or embryos and to increase the regeneration rate in chlorophyllous plants. Using in vitro cultures of unfertilized ovaries, a technique called gynogenesis, we evaluated the effect of 2,4-dichlorophenoxyacetic acid hormonal pre-treatment on production yields and compared it to what we are used to obtain using a cold pretreatment at $4^{\circ} \mathrm{C}$ as previously described in our published works. We applied gynogenesis, on five Moroccan varieties of barley namely Tamelalt, Aglou, Asni, Massine and Arig and compared three variations of gynogenesis protocols. Firstly, we investigated the effect of light and darkness on our cultures. Secondly, we incubated unfertilized ovaries at $4^{\circ} \mathrm{C}$ for 14 days. Thirdly, we injected donor spikes with a solution of 2,4-dichlorophenoxyacetic acid or incubated donor spikes in a solution of 2,4-D at $4^{\circ} \mathrm{C}$ for 14 days. The results obtained show the benefit of darkness over light. We also noted the benefit of 2.4-D pre-treatment in cold compared to a cold pre-treatment alone. Statistical analysis revealed a significant correlation between the genotype and the photoperiod on the outcome of pre-treatments protocols. We hilighted better yields when ovaries were pretreated with 2.4-D and incubated in the light. We also showed that a cold pretreatment alone had no effect for all genotypes and that only ovaries that had undergone a 2,4-D hormonal pretreatment displayed improved induction rates in the Aglou and Asni varieties and improved regeneration rates of in the Asni variety. The latter gave the highest regeneration rate $(13.43 \%)$ when spikes were pretreated by 2.4-D combined with a cold pretreatment and ovaries were placed in light. Moreover, the plants that stemed from this method were stronger. Hence, a hormonal pretreatment using 2,4-D could be an alternative to overcome the hurdles faced when using other types of pre-treatments. Keywords: In vitro culture, Haplomethods, Gynogenesis, Hordeum vulgare L., 2,4-diclorophenoxyacetic acid (2,4-D), Cold.

\section{O5. Some Biochemical Responses of the Use of Arbus-} cular Mycorrhizal Fungi in Date Palm Under Salt Stress

Mohamed Ait El Mokhtar", Said Wahbi, Raja Ben Laovane, Mohamed Anli and Abdelilah Meddich.

Plant Biotechnology and Physiology Lab, Faculty of Sciences, Cadi Ayyad University, Marrakech, Morocco. "Corresponding and Presenting Author: mohamed. aitelmokhtar@gmail.com.

\section{Abstract}

Mycorrhizal and nonmycorrhizal date palm seedlings were subjected to salt stress in order to study some biochemical responses of the effect of arbuscular mycorrhizal fungi (AMF) in alleviation of salt stress. Two months after germination, the plants were mycorrhized and were subjected to $0 \mathrm{mM}$ and $240 \mathrm{mM} \mathrm{NaCl}$ after five months. The activities of two antioxidant enzymes were assessed besides of protein, sugar and polyphenols contents. Activities of peroxidase (POD), and polyphenol oxidase (PPO) and polyphenol content were increased significantly in both mycorrhizal (M) and nonmycorrhizal (NM) plants grown under $\mathrm{NaCl}$ salinity while protein and sugar contents decreased. The results showed that both enzymes activities were very high in roots compared to leaves especially for the PPO. The application of AMF was found to enhance significantly the activities of $P O D$ and PPO under salt stress. In the same conditions, mycorrhizal date palm plants showed a high content on polyphenol in shoots compared to control plant under salt stress. The sugar and protein contents were high in shoots than in roots and under salt stress, the application of AMF improved the both contents. Keywords: Date palm, arbuscular mycorrhizal fungi, salt stress, biochemical responses.

\section{O6. Agricultural Practices in the Souss-Massa Region and their Consequences on Soil Fertility}

Mimouni Abdelaziz*, Bouharrou Rachid, Wifaya Ahmed, Moukrini Fouad, Karra Youssef.

National Institute for Agricultural Research, CRRA, Agadir, Morocco. * Corresponding and Presenting Author: mimouniabdelaziz@yahoo.fr. 


\section{Abstract}

Using a participatory study and monitoring of the history of soil fertility indices, organic matter, total nitrogen, salinity and others, indices of degradation of soil fertility have been identified. Indeed, the cropping system through rotations and techniques practiced in the region have influenced in various ways the soil fertility and subsequently crop performance. Indeed, In the plain of Souss-Massa, agriculture has undergone a great evolution over time, it has reached a level of intensification very high at present. The intensified areas have evolved very rapidly, reaching in 2015 about 56434 ha, mainly in monoculture citrus (39808 ha) and early crops (16626 ha). The significant expansion of these areas was largely the result of the substitution of crops for argan plantations with the dominance of vegetable monoculture, maize and no practice of rotations based on legumes or regenerative soil fertility crops. Over approximately forty years, the clearing of the argan tree and the cultivation of the soil caused a degradation of the organic matter with respective losses of $68 \%$ in the Massa and $57 \%$ for the Souss. Total nitrogen losses were $52 \%$ and $41 \%$, respectively. Also, analyzes of 300 soil samples in different subregions showed that soils in the Souss-Massa region have very low levels of organic matter and total nitrogen. In fact, in the four subregions, Massa, Taroudant, Ait Baha and Taliouine, the samples analyzed showed organic matter contents of less than $0.5 \%$ and absence of samples with contents greater than $2 \% .65 \%$ of the analyzed samples have total nitrogen contents of less than $0.01 \%$. Keywords: Intensified crops, rotations, organic matter, total nitrogen.

\section{O7. Effect of Nitrogen and Seed Germination Rate on Yield, Biomass, and Grain Quality of Moroccan Durum Wheat.}

Oussama Hnizil ${ }^{1^{*}}$, Sedri Naima', Nsarellah Nassele$\mathrm{hq}^{2}$, Baidani Aziz' ${ }^{1}$, Idrissi Omar ${ }^{2}$, Sahri Ali ${ }^{2}$, and Amamou Ali ${ }^{2}$.

${ }^{1}$ University Hassan II, Settat, Morocco; ${ }^{2}$ Not provided. "Corresponding and Presenting Author: o.hnizil@ uhp.ac.ma.

\section{Abstract}

Durum wheat is a typical Mediterranean crop. Its importance comes from the fact that durum wheat grain is consumed in different forms namely bread, pasta, couscous, boulghour... However, these different forms require grains of durum wheat of good quality (protein content, gluten strength, grain color and baking strength). Nitrogen ( $N$ ) element is the nutrient that most frequently limits yield and plays an important role in regulating productivity and quality of durum wheat grain. In the other hand, seed rate (S) is also a vital factor which influence yield and quality of wheat. The objective of this study is to evaluate the effect of different nitrogen treatments and seed rates on yield, total biomass and end use quality of five Moroccan durum wheat varieties. The trial was carried out during the 2017 cropping season, at three experimental stations (Sidi el Aidi, Marchouch and Tassout). The five Moroccan cultivars were sown following split-plot design with two replications, three seed rates (S: 300, 400 and 500 grains $/ \mathrm{m} 2)$ and three nitrogen treatments (N: 45, 90 and $135 \mathrm{~kg} / \mathrm{ha}$ ). Data were recorded for quantitative characters: yield and components, biomass, NDVI, chlorophyll content and grain protein content. With regard to the results, both $\mathrm{N}$ and $\mathrm{S}$ had a significant effect on grain yield, biomass, physiological treats and protein content. In addition, highly significant genetic variation was observed between genotypes and the genetic response to $\mathrm{N}$ supply. The new released varieties showed high response to the variation of $\mathrm{N}$ application. The difference between environments was also observed and discussed. The experiment is carried out also on 2018 to validate the result found during the 2017 cropping season. Keywords: Nitrogen fertilization, planting density, durum wheat, yield and its components, grain quality. 


\section{Concurrent Session V: Plant Health \& Production II}

\section{Co-Chair: Alan Walters, Southern Illinois Univer- sity, USA}

Co-Chair: Mohamed Amine Serghini, University Ibn Zohr, Morocco

\section{O1. Insight into the Mediterranean Olive Tree Domesti- cation and Diversification.}

Ahmed El Bakkali ${ }^{1 *}$, Adelmajid Moukhli², and Bouchaib Khadari ${ }^{3,4}$

1 INRA, UR Amélioration des Plantes et Conservation des Ressources Phytogénétiques, Meknès, Morocco; ${ }^{2}$ INRA, UR Amélioration des Plantes, Marrakech, Morocco; ${ }^{3}$ AGAP, University of Montpellier, CIRAD, INRA, Montpellier SupAgro, Montpellier, France ; ${ }^{4}$ Conservatoire Botanique National Méditerranéen de Porquerolles (CBNMed), UMR AGAP, Montpellier, France. *Corresponding and Presenting Author: ahmed_elbakkali@yahoo.fr.

\section{Abstract}

The olive tree (Olea europaea L.) is one of the most important oil crops in the world and $95 \%$ of total olive oil production derived from the Mediterranean basin (MB). Since the ancient times, out-performed olive genotypes have been selected by local farmers and vegetatively propagated leading to the present varietal assortment with almost 1,200 cultivars around the MB. Therefore, the elucidation of the olive origin, domestication and diversification process is important from a cultural and agricultural standpoint since it can inform on the origin and history of human civilizations as well as the management of genetic resources, while offering guidance for modern breeding. Primary domestication from wild olive probably occurred around 6000 BP in the Middle East. However, the question remains as to whether current cultivated olive derived from single domestication event in the Levant followed by secondary diversification or whether it was the result of independent domestication events. Here we analyzed a comprehensive sample from 35 wild populations (oleasters, 722 individuals) and 410 cultivars from across the $M B$ using nuclear and plastid DNA markers. Our genetic findings from Bayesian model clustering implemented in the STRUCTURE program and maternal lineages argue in favor of a single primary domestication event in the eastern $M B$, followed by diffusion of the first domesticated olive and introgression with wild olive from the central and western MB. These results should be examined to gain further insight as to be able to accurately design sampling of Mediterranean olive germplasm suitable for innovative breeding within the sustainable oliviculture and climate change context. Keywords: Olea europaea, oleasters, simple sequence repeat (SSR), plastid maternal lineage, genetic structure, admixture, single domestication center.

O2. Econometric Analysis of Market Integration and Price Transmission in Morocco's Apples Markets: Application of Threshold Cointegration and Cointegration with Structural Break.

Ouahiba Elalaoui ${ }^{*}$, Abdelouafi Ibrahimy ${ }^{2}$, Aziz Fadlaoui ${ }^{3}$, and Redouane Arrach ${ }^{4}$.

1 Agro-economist engineer, Graduate of the National School of Agriculture; Morocco; ${ }^{2}$ Professor of Applied Statistics and Economics, National School of Agriculture; Morocco; ${ }^{3}$ Agro-Economist Researcher, National Institute for Agricultural Research; Morocco; ${ }^{4}$ Director of Strategy and Statistics Directorate, Ministry of Agriculture, Fisheries, Rural Development, Water and Forests, Rabat, Morocco. "Corresponding and Presenting Author: ouahiba.elalaoui@gmail.com.

\section{Abstract}

The level of market integration and transmission of food prices is a key determinant of price stability and food security. Thus, integration can be regarded as a way of assessing efficiency of agricultural markets. This study investigated the degree of market integration using the time series techniques of threshold cointegration and cointegration with structural break. The purpose of this study is twofold. Firstly, it attempts to determine whether price transmission sufficiently improved throughout the period under examination. Secondly, it purports to explore the nature of price transmission. In order to achieve these objectives, we sampled five wholesale markets, comprising two sur- 
plus markets (Casablanca and Meknes) and three deficit markets (Agadir, Marrakech and Oujda). The dataset for the analysis includes monthly price series from September, 1993 to June, 2017. The results reveal that apple markets are integrated and hence price signals are transmitted from surplus to deficit regions. However, the extent of market integration is low and price transmission is slow and incomplete. Furthermore, the findings indicate that the intensity of price transmission has not improved throughout this period, in spite of the development of road infrastructures and telecommunications. Overall, the empirical evidence confirms the presence of asymmetric price transmission, sign of market inefficiency. Nevertheless, the nature of asymmetry does not confirm the research hypothesis stipulating that increases prices are transmitted more quickly than decreases because of the market power of middlemen in the food marketing chain. Keywords: Market integration, cointegration, apples, price transmission.

\section{O3. Culture Method of Cercospora beticola Sacc, Sen- sitivity Test of Isolates with Methyl Thiophanate and De- tection of Resistance in Morocco.}

Zakariae El Housni ${ }^{1,2^{*}}$, Said Ezrari ${ }^{1,3}$, abdessalem Tahiri ${ }^{1}$, Rachid Lahlali ${ }^{1}$, and Abderrahman Ouiija ${ }^{2}$.

${ }^{1}$ Phytopathology Unit, Departement of Plant Protection, Ecole Nationale d'Agriculture de Meknès, BPS 40, Meknès, Morocco; ${ }^{2}$ Departement of Biology, Faculty of Sciences, University Moulay Ismail, P.O. Box 11201 , Meknes, Morocco. * Corresponding and Presenting Author: atahiri@enameknes.ac.ma.

\section{Abstract}

The Sugar Beet crop (SB) is considered the first source of extraction of refined sugar for Morocco. It is cultivated in four large areas: Gharb, Doukkala, Tadla and Moulouya. The BS has a large list of enemy that significantly reduces yields. Cercospora Leaf Spot $(C L S)$ is the main fungal disease. In the last years, we have observed a decline in efficacy of Methyl Thiophanate (MT), active ingredient belongs of benzimidazole, which was been used extensively in the management of Cercospora Leaf Spot (CLS) in Morocco. Several samples of Cercospora beticola Sacc were collected from the 4 major production areas for labo- ratory tests. Tests on cultivation techniques have been conducted to see if there is an effect of the following factors: The culture media (PDA \& BLA), the light, the sampling area, the method of isolation, on the growth rate of the fungus, the percentage of germination and the percentage of sporulation. With PCR, all isolates were verified by specific primers (CBACTIN959 $\mathrm{F}$ and CBACTIN959 R) encoding $959 \mathrm{bp}$ of the active gene to ensure that the species to study is Cercospora beticola Sacc. Radial mycelia growth of each isolate in PDA medium was compared to mycelia growth in PDA medium modified with MT with levels of $1,5,10$ and $50 \mathrm{ppm}$. The percentage of inhibition obtained was used for the EC50 calculation. All the isolates showed resistance to the MT molecule with different degrees to stand out with 3 groups: Low resistance with EC50 less than 100 ppm, moderate resistant with EC50 between 100 and 1000 ppm and very resistant with EC50 more than 1000 ppm. Keywords: Cultivation techniques, Cercospora beticola, PCR, sensitivity, resistance, Methyl Thiophanate, EC50, Morocco.

\section{O4. A Dynamic Multimarket Analysis of Fat Food Sector in Morocco.}

\section{Sara Essaten ${ }^{1 *}$ and Abdelkader Ait El Mekki ${ }^{2 \S}$}

1 Agronomic and Veterinary Institute, Rabat, Morocco; 2 National School of Agriculture, Meknes, Morocco. "Presenting Author: sara.essaten@gmail.com. ${ }^{\S}$ Corresponding Author: amekki@enameknes.ac.ma.

\section{Abstract}

Due to a severe deficit of the domestic supply, the fat food demand in Morocco is mainly covered by the imports which ensure as much as $80 \%$ of the households' consumption. Thus, Moroccan fat food security is largely defined upon the government efforts to enhance domestic production on one hand, and the world market patterns, on the other hand. In this context, the fat food sector performances should largely be determined by the government price and trade policy knowing that Morocco has signed preferential trade agreements with its main food suppliers (EU and USA) and is an active WTO member. This research aims at analyzing the impact of trade openness and government farm and marketing price policy on the fatty 
products sector in Morocco. In this respect, a dynamic multimarket model, including olive oil, edible oilseed, butter and margarine, will be developed in order to evaluate the effects of price and trade policies related to exogenous variables on the whole fat food value chains from the farmer to the consumer. Indeed, the multimarket model will be made up of six blocks where four of them represent the behavior of economic agents at agriculture, processing, international trade and consumption steps. The other two blocks will be devoted to the market equilibrium and cross price relationship along the value chains. All dynamic behavioral parameters will be econometrically estimated using appropriate microeconomic theory. Keywords: Fat Food Sector, Dynamic multimarket model.

O5. Genetic Variability Revealed by SSR (Simple Sequence Repeat) Markers of Apricot (Prunus armeniaca L.) Issued from Various Genetic and Environmental Resources in Morocco.

Jamal Ayour ${ }^{1,6,7^{*}}$, Imane Elateri ${ }^{2}$, Mohammed Alami ${ }^{3}$, Abderrahim Alahyane', Mohamed Najib Alfeddy ${ }^{4}$, Jean-Marc Audergon ${ }^{5}$, Catherine M.G.C. Renard ${ }^{6,7}$, and Mohamed Benichou'

${ }^{1}$ Faculté des sciences, Université Cadi Ayyad, Laboratoire Sciences des Aliments, M-40090 Marrakech, Morocco; ${ }^{2}$ Laboratory of Molecular Biology and Food, Center of Analysis and Characterisation, Faculty of Sciences-Semlalia, Cadi Ayyad University, P.O. Box: 2390, Marrakesh, Morocco; ${ }^{3}$ Laboratory of Microbiology and Molecular Biology, Faculty of Science, Mohammed V University, Rabat, Morocco; ${ }^{4}$ Laboratoire de Phyto-Bactériologie, Unité de Recherche Protection des plantes au Centre Régional de la Recherche Agronomique, INRA, BP 533, Marrakech, Morocco; ${ }^{5}$ INRA, UR 1052 Génétique et Amélioration des Fruits et Légumes, F-84143 Montfavet, France; ${ }^{6}$ Université d'Avignon et des Pays du Vaucluse, UMR408 Sécurité et Qualité des Produits d'Origine Végétale, F- 84000 Avignon, France; ${ }^{7}$ UMR408 Sécurité et Qualité des Produits d'Origine Végétale, INRA, F-84000 Avignon, France. "Corresponding and Presenting Author: jamal.ayour@ced.uca.ac.ma.

\section{Abstract}

The knowledge of the available genetic resources is of first importance to optimize its preservation and/ or its potential use in breeding programs. The objective of this study was to assess the genetic diversity of Moroccan apricot (Prunus armeniaca L.) accessions available in collection using 21 microsatellite markers. Genotypic diversity of 92 genotypes, issued from a prospection organized in all the country, was determined using diversity indices, molecular variance analysis, principal component and cluster analysis. Polymorphic parameters indicated that the least informative prime was ВРPCTOO1 with 3 genotypes while the most informative primer was UDP98409 with 23 genotypes. The PIC values for SSR loci ranged from 0.08 for AMPA 109 to 0.43 for BPPCTO01, with the mean value of 0.19 . Whereas the Shannon's information index was ranged from 0.37 to 1.94 for AMPA109 and UDP98409 respectively, this shows an importance genetic variability of analyzed apricots regarding their geographic origin. Indeed, the genetic variance was about $87 \%$ among apricot clones and $10 \%$ among geographic region. The results of neighbour-joining, tree analysis and PCOA indicated that the genetic structure of the 92 apricot clones could be grouped in different populations according to their agronomic performances. Information obtained in this study may be useful for apricot breeding improvement programs and it could enable the enlargement of the apricot genotype in the Mediterranean basin scale. Keywords: Prunus armeniaca, clone, genetic diversity, microsatellite markers, variability.

\section{O6. Genetic Diversity of Moroccan Sesame (Sesamum Indicum L.) Populations Using ISSR Markers}

Meriem El Harfi ${ }^{1,2^{*}}$, Abdelghani Nabloussi', Hafida Hanine $^{2}$, and Jamal Charafi ${ }^{1 \S}$

1 Plant Breeding and Genetic Resources Unit, National Institute of Agronomic Research, Regional Agricultural Research Centre of Meknes, Morocco; ${ }^{2}$ Laboratory of Bioprocess and Biolnterfaces, Faculty of Science and Technology, Beni Mellal, Morocco. "Presenting Author: elharfi.meriem@gmail.com. §Corresponding Author: jcharafi@gmail.com.

\section{Abstract}

Sesame (Sesamum indicum L.) is an ancient oilseed crop known for its nutty seeds and high-quality edible oil. 
It is an unexplored crop with a great economic potential. In Morocco, Sesame has been grown for decades and it is one of the specific crops of the Tadla-Azilal area. The present study deals with assessment of genetic diversity among 33 sesame populations. Seven Inter Simple Sequence Repeat (ISSRs) primers were used for molecular analysis of these populations. A total of 57 markers were detected and the number per primer varied from 4 to 14 , with an average of 8 bands. The primers generated $82 \%$ (47 bands) of polymorphic fragments and the pairwise comparison of the 33 genotypes showed that $74 \%$ of genotypes pairs were distinct by less than 11 markers. This low diversity between genotypes is confirmed by the genetic distances obtained. The Simple Matching coefficient of Similarity ranged from 0.509 to 1 , with an average of 0.79 and the polymorphic information content (PIC) ranged from 0.002 to 0.349 . The hierarchical analysis using the UPGMA method showed that, despite subdivision into groups, several genotypes had the same genetic profile and were lowly diversified. The results obtained show that sesame in Morocco has a low genetic diversity and may be probably derived from a limited genetic pool. Keywords: Sesamum indicum L., ISSRs Markers, Genetic diversity, Polymorphism.

\section{O7. Effect of Moroccan Seaweed Polysaccharides on Germination of Tomato Seeds (Solanum lycopersicum L.).}

Abir Mzibra ${ }^{1,2^{*}}$, Abderrahim Aasfar ${ }^{1}$, Issam Meftah Kadmiri' ${ }^{1}$, and Ahmed Bamouh ${ }^{2}$

1 Laboratory of Green Biotechnology of Moroccan Foundation for Advanced Science, Innovation and Research (MAScIR), Rabat Design Centre, Rue Mohamed Al Jazouli, Madinate Al Irfane, Rabat, Morocco; ${ }^{2}$ Institut Agronomique et Vétérinaire Hassan II, Production, Protection et Biotechnologie Végétales, BP: 6446, Rabat, Morocco. "Corresponding and Presenting Author: abiir.mzi@gmail.com.

\section{Abstract}

Polysaccharides extracted from marine seaweeds can act as plant biostimulants by applying them to seeds, plants, or growing substrates, but always at low concentration, which is provided by specific for- mulations. The aim of this study was to evaluate the effect of Polysaccharides Enriched Extracts (PEEs) obtained from 17 Moroccan seaweeds belonging to Chlorophyceae (5 species), Phaeophyceae (5 species) and Rhodophyceae (7 species) on 3 germination parameters: Germination Percentage (GP), Germination Speed (GS) and Mean Time of Germination (MGT) of tomato seeds (Solanum lycopersicum) under laboratory conditions. Tomato seeds were cultivated in The Half-strength Murashige-Skoog medium and supplemented with different PEEs at 3 concentrations of $(0.1 ; 0.05$ and $0.02 \mathrm{mg} \mathrm{mL}-1(\mathrm{w} / \mathrm{v})$ or with sterile distilled water for control seeds. Our results showed that seeds treated with PEEs extracted from U. rigida, C. tomentosum, C. decorticatum, Gigartina sp., B. bifurcata, F. spiralis, S. polyschides in the 3 concentrations enhanced germination (significant increase in germination percentage associated with lower significant mean germination time and high significant germination speed) and seeds treated with PEEs of G. pistillata, C. acicularis, G. crinale, C. foeniculacea and $C$. tamariscifolia enhanced significantly germination parameters just at lower concentrations (0.02 mg.mL-1). G. pistillata at higher concentration (0.1 mg.mL-1) showed an inhibition of germination. This study emphasizes the use of Moroccan PEEs as biostimulants inducing an early tomato seed germination and establishment with the corresponding concentration. Keywords: Biostimulant; Polysaccharides enriched extracts; Seaweed; Seed germination, Germination parameters.

\section{O8. Ethnobotanical uses of Arbutus (Arbutus unedo L.) in Morocco.}

Faida Rahima $\frac{1,2^{*}}{}$, J Aabdousse' ${ }^{1}$ A Boulli', S Bouda ${ }^{2}$, and $\mathrm{N}$ Wahid ${ }^{1 \S}$

1 Laboratory of Environment and Valorization of Agro-Resources (EVAR), Department of Life Sciences, Faculty of Science and Technology, Sultan Moulay Slimane University, Beni-Mellal, Morocco; ${ }^{2}$ Laboratory of Management and Valorization of Natural Resources, Department of Life Sciences, Faculty of Science and Technology, Sultan Moulay Slimane University, Beni-Mellal, Morocco. "Presenting Author: faida.rahima@gmail.com. ${ }^{\S}$ Corresponding Author: wahid2na@ hotmail.com. 


\section{Abstract}

Arbutus is a fruit tree with high ornamental, environmental, economic and medical value, because of the properties attributed to different biological parts. Given this interest, the present study is interested in describing and compiling available data on the ethnobotanical uses of Arbutus in Morocco in comparison with other studies at the international level. It is very present in the everyday uses of foresters, farmers and rural populations. The international bibliographic analysis shows that the different biological parts of Arbutus, especially fruits, have been widely used by herbalists. The related literature is generally in line with the medicinal use of the plant by Moroccan inhabitants. On the other hand, the consumption and the industrial valorization of the fruits of Arbutus are very limited in Morocco in comparison with those on the international scale. It is imperative to sensitize the local population and landlocked Dovars on the knowledge of the valorization of the fruit of this species. Keyswords: Arbutus unedo L., ethnobotanical uses, prospection, ecology, Arbutus fruit.

O9. Valorization Challenges to Almonds and their Coproducts: Characterization of Oils Extracted from Broken Almonds Springing from Shelling and Sorting Operations.

Ahmed Elamrani ${ }^{1^{*}}$, R. Melhaoui, N. Houmy, M. Addi; M. Abid, A. Mihamou, M-L. Fauconier ${ }^{2}$, M. Sindic ${ }^{3}$, and Serghini-Caid'

1 Laboratoire LBPM, Faculté des Sciences, Université Mohamed ler, Oujda; Morocco; 2 Laboratoire de Chimie Générale et Organique, Gembloux Agro Bio-Tech, Université de Liège; Belgium; ${ }^{3}$ Laboratoire, QSPA, Gembloux Agro Bio-Tech, Université de Liège; Belgium. "Corresponding and Presenting Author: ahmed.elamrani@gmail.com.

\section{Abstract}

The almond tree (Prunus dulcis) is one of the most popular nut trees grown worldwide under arid conditions. In north eastern region of Morocco, after the olive tree the almond tree is the second most important tree crop in this region, due to its resistance and capacity against water shortage and irrigation defi- cit. During this decade, new orchards of almond trees have emerged, thanks to the program Green Morocco, supported by the BTC ("BTC-PROFAO project). Thus 6.000 hectares of new orchards of almond trees were planted using the association Ferragnes / Ferraduel, which is a couple of French cultivars known for their late blooming. In the aim to improve the income of this rural population, cooperatives and economic interest groups were created; farmers have been trained for good almond cultivation practices as well as almond harvesting and processing. In eastern Morocco, traditionally almonds are hulled and shelled, manually by rural women, but currently, almonds processing machines are under installation. Manually or mechanically, almonds' hulling and shelling operations generate many byproducts which are hulls (thin mesocarp, green shell cover), shells (Hardened endocarp) but also broken kernels from almonds sorting and brown skin as byproduct of almonds' blanching. Taking in consideration the importance of valorization of almond byproduct, this work deals with the agro economical relevance and added value that could be generated by using broken almonds (as a co product) for Almond oils extraction. Thus this oral communication focuses on (i) the characterization of Almond oils produced by cooperatives in this region as an adding value to this almond's co-products (ii) Paths of research for the valorization of the other by-products mainly almonds' shells and hulls. Briefly, almond oils were extracted from broken almond (as a co-product) by screw press, oil yield rage between 48 and $56 \%$. Fatty Acids (FA) profile, determined by GC-FID, shows dominance of unsaturated fatty acids fraction [USFA $>85 \%$ ] which is mainly represented by oleic Acid (61-69\%) and linoleic acid (22-27\%). Saturated fatty acids fraction (SFA $<11 \%$ ) is represented mainly by palmitic acid (7-8\%). and stearic acid (2$3 \%$ ). Total phenol content range between $40-45 \mathrm{mg} /$ $\mathrm{kg}$-oil, however total tocopherols content is important and range between $510-530 \mathrm{mg} / \mathrm{kg}$-oil with a large dominance of $\alpha$ tocopherol. The presence of these compounds is important in relation to oil stability and as nutritional quality label. Thus, consumption of almond oil is recommended thanks to their beneficial effects on health, but it's also recommended for cosmetic uses. Keywords: Almond Tree, Almonds, almond co-products, Almond Oils.

"BTC- PROFAO: Projet Filière Amandes de L'Oriental, "The almond value chain in eastern Morocco, Pillar II 
of the Moroccan Green Plan, supported by Belgian development agency (BTC 2011-2017).

\section{Session VI: Africa Sustainable Agricul- ture (Plenary Session)}

\section{Chair: Khalid Meksem, Southern Illinois Univer- sity, USA}

\section{A Strategy for Incorporating Sustainability into Ter- tiary Agriculture Education.}

\section{John W. Groninger ${ }^{*}$}

Department of Plant, Soil, and Agricultural Systems, Southern Illinois University, Carbondale, IL 62901, USA. "Corresponding and Presenting Author: groninge@siu.edu.

\section{Abstract}

Where agriculture production systems are rapidly evolving, such as across sub-Saharan Africa, shortterm gains in crop yields can occur at the expense of system resiliency needed to achieve sustainability. Post-secondary (tertiary) education institutions have great potential to better facilitate large scale sustainability initiatives. Agricultural education resources that do not reflect present industry conditions and the declining prestige of agriculture in urbanizing societies further hinders reform efforts. Based on a 2014 assessment of Samaru College of Agriculture at Ahmadu Bello University in Nigeria (funded by the United States Agency for International Development Farmer to Farmer program and implemented by Winrock International), I identify strategies for using increasing interest in agricultural sustainability to revitalize Agriculture Teaching institutions by broadening their value to students and better demonstrating the relevance of agriculture institutions to society. My presentation will focus on education system reforms that can address watershed management issues. Particularly critical are curriculum reforms that promote entrepreneurship, build creative problem-solving skills, and encourage meaningful industry engagement among students and staff alike. I conclude with suggestions for increased engagement among uni- versities in Morocco and the United States to work cooperatively with colleagues in sub-Saharan Africa to address ongoing and emerging agriculture sustainability issues among all parties.

\section{Assessing the Impacts of Agroforestry on Commu- nity and Household Resilience in Africa: An Analysis of the Modified Taungya System in Ghana}

\section{Kofi Akamani*}

Department of Forestry, Southern Illinois University, Carbondale, IL 62901, USA. "Corresponding and Presenting Author: k.akamani@siu.edu.

\section{Abstract}

The promotion of sustainable development on the African continent continues to be challenged by several problems, including population pressure, widespread poverty, food insecurity, resource degradation, and climate change vulnerability. Owing to the shortfalls of industrial agriculture and sustained-yield forest management approaches, agroforestry has been receiving attention as an integrative land use strategy for achieving the Sustainable Development Goals in Africa. Agroforestry, a land use strategy that involves the integration of trees with annual crop cultivation and other farm activities, promises several benefits including food security, ecosystem restoration, as well as climate change mitigation and adaptation. However, the relationship between agroforestry and community resilience, i.e. the capacity of communities to adapt to change while maintaining their well-being, has not received adequate attention in the literature. Importantly, the issue of scale has not received explicit recognition in the assessment of agroforestry outcomes. Since the adoption of the Modified Taungya System (MTS) in Ghana in the early 2000s, a collaborative approach to agroforestry has been pursued as a means for forest restoration, as well as food and timber production. This presentation utilizes qualitative and quantitative data gathered from two forest-dependent communities in the Ashanti region of Ghana to assess the impacts of the MTS program on social-ecological resilience at the level of the community and the household. The purpose is to determine whether the impacts of the MTS program on socialecological resilience at the community and household 
levels are equivalent to each other. Analysis of qualitative data at the community level indicated that when comparing current community conditions to conditions prior to the implementation of the MTS program, both communities have experienced marginal improvements in the various capital assets that shape community resilience. At the household level, a statistical comparison of current household capital assets with capital assets prior to the MTS program showed varying levels of decline in household resilience across the two communities. These results suggest the impacts of agroforestry on social-ecological resilience may be sensitive to the scale at which assessments are made. Keywords: Agroforestry, community resilience, livelihoods, sustainable development, scale.

\section{O3. The Future Agriculture in Africa in the Context of Climate Change: Seeds Perspective}

Lamiae Ghaouti*. Institut Agronomique et Vétérinaire Hassan II, Rabat, Morocco. "Corresponding and Presenting Author: lamiaeghaouti@hotmail.com.

\section{Abstract}

Agriculture is highly exposed to climate change, as farming activities directly depend on climatic conditions. The modern agriculture through the maximization of natural resources use contributed in a considerable proportion to the climatic change through the release of greenhouse gases into the atmosphere. In comparison to the other continents, Africa is the least contributing to the carbon dioxide emissions but is paradoxically the most impacted area in the world by climatic changes. Therefore, rethinking the agricultural model in Africa where agriculture is a crucial sector is a complex issue but compulsory within the view of the present situation. Africa holds a relevant asset through genetic diversity as it is a center of diversity for a wide range of crops. It is an essential component in the equation of maintaining the food production level while decreasing the agro-ecosystems vulnerability. Genetic diversity is a key factor for the management of heat, drought, salinity, pests and diseases that are more acute due to climatic changes. Cropping systems should be adopted depending on their efficiency of use of natural resources within the targeted agro-ecosystems. Rethink the agricultural production paradigm. Seed sector in Africa which is dominated essentially by an informal system constitute a potential leverage to enhance productivity and encounter climatic changes. For this purpose, actions should be defined for the promotion of both the formal and the informal sector within their respective contexts. Keywords: Africa, climatic change, agricultural model, seed sector.

\section{O4. Status of Vegetable Variety Improvement for Mo- rocco}

\section{Alan Walters ${ }^{*}$}

Department of Plant, Soil, and Agricultural Systems, Southern Illinois University, Carbondale, IL USA. 'Corresponding and Presenting Author: awalters@siu.edu.

\section{Abstract}

The choice and utilization of vegetable varieties by growers depends upon many different factors, and the dynamics leading to eventual variety choice and use is highly varied, even in Morocco. The globalization of agriculture, along with the privatization of the plant breeding industry in the last few decades, has directly influenced which vegetable varieties are available to farmers in developing countries. However, new vegetable variety development is normally non-existent in developing countries, with seed generally sourced from developed countries. This results in developing countries having a dependence on those developed countries that have bred the varieties to supply vegetable seed for their needs. Morocco's goal for future vegetable market development is to produce high-quality products to be sold in international markets that allow the highest revenue returns, and this generally requires the use of hybrid varieties that were not necessarily developed for the specific environments in which they are being recommended. So, this situation often times results in vegetable seeds being marketed into regions having environments where they are not always best suited to maximize their productivity. Thus, the adoption of modern hybrid varieties has lagged in some regions, especially marginal environments that have overall low production potential, which are often most suitable for local traditional varieties (landraces). New variety development works best if breeding is conducted in the environment in which it will be grown to maximize 
future crop yield and productivity. Therefore, the development of new varieties specific to environments in developing countries is a critical step for future food security in these regions. Keywords: Agricultural globalization; Climate change; Food security; Landraces.

\section{Concurrent Session VII: Plant Health \& Production III}

\author{
Co-Chair: John Groninger, Southern Illinois Uni- \\ versity, USA \\ Co-Chair: Fouad Mokrini, INRA, Morocco
}

O1. Resistance to Pratylenchus penetrans and P. thornei in International Wheat Lines and its Durability When Inoculated together with the Cereal Cyst Nematode Heterodera avenae, Using qPCR for Nematode Quantification

Fouad Mokrini ${ }^{1 *}$, Nicole Viaene ${ }^{2,4}$, Lieven Waeyenberge $^{2}$, Amer Dababat $^{5}$, and Maurice Moens ${ }^{2,3}$

1 National Institute for Agricultural Research (INRA), Rue FAR, Inzegane, Agadir, Morocco; ${ }^{2}$ Institute for Agricultural and Fisheries Research, Plant, Crop Protection, Burg. Van Gansberghelaan 96, B-9820 Merelbeke, Belgium; ${ }^{3}$ Faculty of Bio-Science engineering, Ghent University, Coupure links 653, B-9000 Ghent, Belgium; ${ }^{4}$ Department of Biology, Ghent University, Ghent, Belgium; ${ }^{5}$ International Maize and Wheat Improvement Center (CIMMYT), P.K. 390651 1, Emek, Ankara, Turkey. ${ }^{*}$ Corresponding and Presenting Author: fmokrini.inra@gmail.com.

\section{Abstract}

The root lesion nematodes Pratylenchus penetrans and $P$. thornei cause high yield losses in rainfed wheat fields in Morocco, as well as worldwide. Growing resistant varieties is one of the most effective methods for controlling nematodes. Therefore, a collection of 14 lines of spring wheat and 11 lines of winter wheat (Triticum aestivum and T. durum), developed at CIMMYT, were screened for resistance to P. penetrans and P. thornei in tubes $(15 \times 20 \times 120 \mathrm{~mm} 3)$ under the greenhouse conditions. The resistance level was evaluated based on the numbers of nematodes extracted from roots and soil nine weeks after infestation. The number of P. penetrans or P. thornei were determined visually using a microscope and with a qPCR assay. Three lines (L9, L1 2 and L13) were found moderately resistant to P. thornei and one of these (L9) was also moderately resistant to $P$. penetrans. To investigate the durability of this resistance, we co-inoclulated juveniles of Heterodera avenae, a cereal cyst nematode widely present in Moroccan wheat fields, and assessed the reproduction of both lesion nematodes P. penetrans and P. thornei on the three moderately resistant lines. Our results showed that the lines $L 9$ and $L 9, L 12, L 13$ remained moderately resistant to P. penetrans and P. thornei, respectively, in the presence of $\mathrm{H}$. avenae. These findings are promising; however, the field performance of these lines against root lesion nematode attacks should be evaluated. Keywords: durability, resistance, root lesion nematode, screening, wheat.

O2. Transfer and Molecular Mapping of Aegilops tauschii-derived Hessian Fly Resistance Genes (H22, H23, H24, and H26) from D Genome of Triticum aestivum onto A Genome Chromosomes of Triticum turgidum by Induced Homoeologous Recombination.

Moha Ferrahi ${ }^{1^{*}}$, B. Friebe ${ }^{2}$, J.H. Hatchett ${ }^{3}$, and B.S. Gill ${ }^{2}$

1 National Institute for Agricultural Research (INRA), Regional Center of Meknes, BP 578, Meknes, Morocco; ${ }^{2}$ Dept. of Plant Pathology, Wheat Genetics Resource Center, Throckmorton Plant Sciences Center, Kansas State University, Manhattan, KS 66506-5502, USA; ${ }^{3}$ Dept. of Entomology and USDA-ARS, Waters Hall, Kansas State University, Manhattan, KS 66506 5502, USA. "Corresponding and Presenting Author: mohaferrahi@yahoo.fr.

\section{Abstract}

Aegilops tauschii Coss. $(2 n=14, D D)$ is a rich source of disease resistance genes for the improvement of cultivated wheat including several resistance genes against Hessian fly. To date, five Hessian fly resistance genes $(\mathrm{H} 13, \mathrm{H} 22, \mathrm{H} 23, \mathrm{H} 24$, and $\mathrm{H} 26)$ have been transferred from Ae. tauschii to common wheat (Triticum aestivum L.). In this study, we attempted the trans- 
fer of four genes H22 (1D), H23 (6DS), H24 (3DL), and H26 (4D) from T. aestivum D genome onto A genome chromosomes of T. turgidum. The T. aestivum resistant parents WGRCO1 (H22 on 1D), WGRCO3 (H23 on 6DS), WGRC06 (H24 on 3DL), and WGRC26 (H26 on 4D) were crossed with $\mathrm{T}$. turgidum $\mathrm{cv}$. Langdon disomic substitution lines LDN 1D(1A), LDN 6D(6A), LDN $3 D(3 A)$, and $L D N 4 D(4 A)$. We targeted the transfer of Hessian fly resistance genes into D-genome substitution chromosomes of T. turgidum by homologous recombination. In total 88 crosses were made. The resulting F1 plants (345 seeds) were backcrossed with the LDN 5D(5B) substitution line in which chromosome $5 \mathrm{~B}$ is absent and replaced by a pair of $5 \mathrm{D}$ chromosomes with the objective of transferring $D$ genome Hessian fly resistance genes onto $A$ or $B$ genomes of T. turgidum by homoeologous recombination. A total of 2,053 segregating $B C 1 F 1$ plants were tested for Hessian fly resistance, and the resistant plants $(1,132)$ were backcrossed again with $L N D 5 D(5 B)$ to produce $B C 2 F 1$ and selfed to produce $B C 1 F 2$. In the $B C 1 F 1$ populations, 24 families segregated for an excess of resistant plants than the expected 1:1 resistant to susceptible plants suggesting that they were putative A-D genome positive recombinants. Mapping analysis using microsatellites was used in these families to identify recombinants between A- and D- genome chromosomes. The data indicated that $\mathrm{H} 22$ recombinants were recovered consisting of the distal part of the short arm of 1A, the proximal of 1DS, and the complete long arm of 1D. The recombinant can be described as T1AS-1DS.1DL. The recombinant involving $\mathrm{H} 23$ probably consisted of the whole short arm of $6 \mathrm{D}$ and the long arm of $6 \mathrm{~A}$, and is described as T6DS.6AL. The centromeric marker indicated that this recombinant has the centromere from chromosome 6A. In addition, monosomic substitution lines were recovered for the remaining resistance genes $\mathrm{H} 24$ and H26. These monosomic substitution lines are useful germplasm for further manipulation aimed at transferring genes $\mathrm{H} 24$ and $\mathrm{H} 26$ to durum wheat. Keywords: Triticum turgidum, Triticum aestivum, Aegilops tauschii, Hessian fly, induced homoeologous recombination, microsatellites mapping.
O3. Analysis of Genetic Diversity Among Safflower Accessions of Different Origins Using Agro-Morphological Traits and ISSR Markers

Karim Houmanat $\frac{1,2^{*}}{}$, Jamal Charafi', Hamid Mazouz ${ }^{2}$, Mohamed El Fechtali', and Abdelghani Nabloussi ${ }^{1 \S}$

1 INRA, Research Unit of Plant Breeding and Plant Genetic Resources Conservation, CRRA of Meknes, P.O. Box 578, Meknes, Morocco; ${ }^{2}$ University Moulay Ismail, Laboratory of Biotechnology and Molecular Biology, Faculty of Sciences, Meknes, Morocco. "Presenting author: k.houmanat@gmail.com. ^Correspomding Author: abdelghani.nabloussi@gmail.com.

\section{Abstract}

Safflower (Carthamus tinctorius L.) is one of the oilseed crops that have important agronomic and environmental benefits, mainly adaptation to arid and semi-arid environmental conditions. This enables it to be a good alternative oilseed crop for Morocco and other Mediterranean countries which are exposed to an increased climate change trend. The present study aimed to evaluate 61 safflower accessions, from different geographical origins, for agronomic, morphological, phenological, pathological and technological attributes. In addition, molecular analysis, using ISSR markers, was carried out to characterize these accessions which were planted in two consecutive years, 2013 and 2014, in INRA-Experimental Station of Douyet. Results obtained for agro-morphological study exhibited a large variability among the genetic material evaluated. Hence, average seed yield was about 2 t/ha, with a variation from 1.14 to $4.3 \mathrm{t}$ / ha. Seed oil content varied between $22 \%$ and $39 \%$. Significant differences were also observed among the accessions for their flowering time, their resistance to brown rust and broomrape, for the color of the petals and for their spinesness. Results of molecular analysis showed that a high number of reproducible ISSR bands exhibited a high percentage of polymorphism, indicating a high genetic diversity among the material studied. Polymorphic profiles were identified according to an index of genetic diversity, generally variable according to the genotypes, from 0.13 to 0.40 , with an average of 0.23 . Dendrogram similarity relationships revealed some clustering according to different genetic pools. The ascending hierarchi- 
cal classification (AHC) of molecular data showed two large distinct groups and four subgroups, whilst AHC of agro-morphological data revealed two large distinct groups with a very high level of similarity (0.977). By analyzing and comparing both $A H C$, one could observe that classification of some accessions in one group is almost similar for both dendrograms. The findings of this study were very interesting and would be useful for safflower breeding program in Morocco as well as in other countries of the world. Keywords: Genetic Resources, Safflower, Variability, Genetic Pools.

\section{O4. Assessment of Codling Moth Sensitivity (Cydia po- monella L.) to Certain Insecticides Used in Apple Or- chards in Morocco, Azrou Region}

Salma El Iraqui ${ }^{1^{*}}$, Ahmed El Bakkali', and M'Hamed Hmimina $^{2 \S}$

1 National Institute of Agricultural Research, Meknès Regional Center, BP 578, Meknès, Morocco; ${ }^{2}$ Zoology Department, Institut Agronomique et Véterinaire Hassan II, B.P. 6202, Rabat, Morocco. *Presenting Author: iraquisalma@gmail.com. ${ }^{\S}$ Corresponding Author: hmimina@yahoo.fr.

\section{Abstract}

The codling moth, Cydia pomonella (L.), is the key pest of apple production worldwide. In Morocco, there is a sustainable presence of codling moth causing considerable damage in apple orchards despite frequent applications of broad spectrum insecticides. At Azrou region (Morocco), the chemical control is widely and massively used ( 12 to 15 treatments per season) exclusively to control this pest. This technique seems to be in the advantage of the insect since the traps captures are usually exceeding the action threshold. The failure of codling moth control in the Azrou orchards led us to examine the insect sensitivity to some insecticides and explore the mechanisms implied in their detoxification. The efficacy of azinphos-methyl, chlorpyriphos-ethyl, diflubenzuron, thiacloprid, methoxyfenozide, spinosad, and deltamethrin, was evalvated on neonate larvae and compared with a laboratory sensitive strain. Toxicological tests showed that the populations tested are developing a resistance. In fact, biotests established a decrease of sensitivity for
5 products out of 7 and exhibited the presence of a cross-resistance between the organophosphates, the benzoylureas, the pyrethroids, the neonicotinoids and the diacilhydrazines. This resistance is mainly linked to the esterase activity unlike the mixed-function oxidase activity which was the same between local and sensitive strains. Finally, the kdr mutation, linked to deltamethrin resistance, was found with a low frequency in Azrou population attesting that resistance is being selected there.Keywords: Cydia pomonella $\mathrm{L}$, sensitivity, resistance, enzymes, esterase, mixed-function oxidase, $\mathrm{kdr}$.

\section{O5. Using Amplicon Sequencing Analysis to Decipher the Microbiome for Date Palm "Bayoud" Disease Sup- pression in Suppressive Soils}

\section{Adil Essarioui ${ }^{1 *}$, Daniel Cameron Schlatter ${ }^{2}$, Harold} Corby Kistler ${ }^{3,4}$, and Linda Kinkel ${ }^{4}$

${ }^{1}$ National Institute for Agricultural Research, Regional Center of Errachidia, Morocco; ${ }^{2}$ USDA, Washington DC, USA ${ }^{3}$ Cereal Lab Disease, USDA-ARS, St Paul, $M N$, USA; ${ }^{4}$ Department of Plant Pathology, University of Minnesota, USA. "Corresponding and Presenting Author: essar002@umn.edu.

\section{Abstract}

"Bayoud", a vascular disease caused by the soilborne fungus Fusarium oxysporum f. sp. albedinis, is the principal enemy of date palm trees that put at stake the future of date industry in Morocco. Previous work showed that some soils are naturally suppressive to the disease. However, our knowledge of how Bayoud pathogen is suppressed in these soils is still limited. To improve our understanding of mechanisms by which "Bayoud" is suppressed in suppressive soils, we characterized fungal and bacterial community composition and structure in suppressive and conducive soils using amplicon sequencing analysis. Fungal and bacterial populations were analyzed by amplifying and Illumina sequencing the internal subscribed spacer (ITS1) and the ribosomal (16S) genes, respectively. Results showed that the suppressive soils have greater microbial density and diversity than conducive soils. Additionally, suppressive soils are more enriched in microbial taxa known for their prolific production of antimicrobial compounds such as Fusarium, Aspergil- 
lus, and diverse Actinomycetes. We hypothesize that "Bayoud" suppression is the results of two complementary mechanisms: competition for nutrients between the pathogen and a highly dense saprophytic microbial community, and antagonism from antibiotic producing microbial taxa. Keywords: Bayoud disease, suppressive soils, amplicon sequencing.

\section{O6. Genome Wide Association Studies on Yield Compo- nents Using a Lentil Genetic Diversity Panel}

Jamin A. Smitchger', Yu $\mathrm{Ma}^{2}$, Ping Zheng ${ }^{2}$, Clarice J. Coyne ${ }^{3^{*}}$, Rebecca J. McGee ${ }^{3}$, and Doreen Main ${ }^{2}$

1 Department of Crop and Soil Sciences, Washington State University, Pullman, WA 99194; ${ }^{2}$ Department of Horticulture, Washington State University, Pullman, WA 99194, USA; ${ }^{3}$ United States Department of Agriculture, Pullman, WA 99194, USA. *Corresponding and Presenting Author: Clarice.Coyne@ars.usda.gov.

\section{Abstract}

The cool season food legume research community are now at the threshold of deploying the cutting-edge molecular genetics and genomics tools that have led to significant and rapid expansion of gene discovery, knowledge of gene function (including tolerance to biotic and abiotic stresses) and genetic improvement of many crop species. We employed two-enzyme genotyping-by-sequencing to genotype a 367 accession lentil diversity panel (USDA Lentil Core Collection) for use in genome wide association studies for yield components. The SNP markers were filtered for a minimum site count of $75 \%$ and minimum allele frequency of 0.05 resulting in 4,084 total SNPs in the final data set. We will present preliminary SNP marker discovery based on one year of field phenotyping for days to $50 \%$ flowering, plant height, height to lowest pod, average seed per pod, pod dehiscence, $90 \%$ maturity and seed yield per plant.

\section{Concurrent Session VIII: Micropropa- gation \& Tissue Culture}

\author{
Co-Chair: Abdelghani Nabloussi, INRA, Moroc- \\ co
Co-Chair: Kofi Akamani, Southern Illinois Uni- versity, USA

\section{O1. In Vitro Micropropagation of Almond Rootstock}

Soufana Safih ${ }^{1,2^{*}}$, A. Bakkali' , N. Ben Bassou' ', K. Makroum $^{2}$, and M. Belfaiza ${ }^{2}$

1 Unité de Recherche Amélioration des Plantes et Conservation des Ressources Phytogénétiques; Centre Régional de Recherche Agronomique de Meknès, Morocco; ${ }^{2}$ Laboratoire de Biotechnologie Végétale, Ecologie et Valorisation des écosystèmes, Université Chouaib Dokkali, El Jadida, Morocco. "Corresponding and Presenting Author: soufana.safih@gmail.com.

\section{Abstract}

almond tree can produce in difficult conditions such as drought, salinity... due to the rootstock resistance and tolerance. Many micropropagation protocols were developed for almond rootstock; It's a suitable and fast method for obtaining a large number of genetically identical plants. The objective of the present work was to evaluate different protocols of almond micropropagation rootstock under the same condition to see the more efficient method. In shoot multiplication, two mediums were evaluated MS (Murashige \& Skoog 1962) and QL (Quoirin \& Lepiovre, 1977) Supplemented with variable combination of IBA and BAP. the best shoot proliferation was observed through the mean number of shoots and the mean shoot length; To see the efficient protocols to increase the shoot length MS was added with BAP and with GA separately. Rooting is considered a critical stage in Prunus sp. Micropropagation; The routing effect of various concentrations of IBA and ANA added to $1 / 2$ MS medium was compared; The best method corresponds to the highest in vitro rooting rate as well as root number per shoot. Keywords: Prunus, micropropagation, rootstock, almond. 


\section{O2. Micropropagation of local "Beldi" Almond Eco- types}

Mohamed Addi ${ }^{*}$, S. Kodad, R. Melhaoui, H. Serghini, A. Elamrani, A. Mihamou, and M. Abid

LBPM Laboratoire, Faculté des Sciences, Université Mohammed Premier, Oujda, Morocco. *Corresponding and Presenting Author: mohamedaddi78@gmail. com.

\section{Abstract}

The almond tree, after the olive tree, is the fruit-bearing species, which occupies the most surface in Morocco. In the Eastern region of Morocco, the almond tree occupies a surface of 26,000 ha producing 14 to $15 \%$ of the national production out of almonds (1). Many native almond trees (local ecotypes) are still grown in the north eastern region of the country and new species have been introduced particularly cultivars imported from France and Spain. Local genetic resources have not yet been systematically conserved. The conventional methods of maintaining fruit-tree crops, both of local ecotypes and introduced cultivars, for germplasm preservation for long periods require extensive space and labour. Tissue culture has been exploited to maintain genetic variability of local ecotypes from which crop plants could be improved, to produce healthy planted materials and to increase the number of desirable germplasm useful for almond breeding program (2). The present study was performed to determine the best combination of some growth regulators for the in vitro germination of isolated zygotic embryos in newly harvested Beldi almond seeds. Preliminary results showed multiple shoot initiation on MS medium containing 30 gl- 1 sucrose, $1 \mathrm{mgl}-1$ BAP and 8 gl-1 agar. Keywords : Micropropagation, almond, Beldi ecotypes, genetic variability, tissue culture.

(1) Veille économique-Secteur amandier 2017, Minisère de l'agriculture et de la pèche maritime. http://www.agriculture.gov.ma/pages/rapportsstatistiques/campagne-agricole-2015-2016

(2) Conservation of Biodiversity Through Tissue culture Sujata Mathur Research and Reviews: Journal of Microbiology and Biotechnology Volume 2 | Issue 3 | July - September, 2013.

\section{O3. Demonstration of the Neutralizing Effect of Lumbri- cus terrestris on $\mathrm{pH}$}

\section{Abdellatif El Harti and Mohammed Raovane* ${ }^{*}$}

Reserch Team : Lumbricidae, Improving Soil Productivity and Environment (LAPSE), Centre "I Eau, Ressources Naturelles, Environnement et Développement Durable (CERN2D) " Mohammed V University in Rabat, Ecole Normale Supérieure (ENS), Avenue Mohamed Belhassan El Ouazani, B.P. 511 8, Takaddoum, Rabat, Morocco. "Corresponding and Presenting Author: mohamed_raovane@yahoo.fr.

\section{Abstract}

This study attempts to clarify the role of Lumbricus terrestris in the regulation of soil $\mathrm{pH}$. The experimental approach adopted for this study involved using fasting worms following a totally in vitro protocol, which made it possible to rule out all possible interference with the soil components. Immersion of the worms in the reaction solutions at different $\mathrm{pHs}$ allowed the delimitation of a tolerance zone between $\mathrm{pH}=2$ and $\mathrm{pH}=12$. In this zone, the more the $\mathrm{pH}$ deviates from the neutrality $(6 \leq \mathrm{pH} \leq 8)$, the more intense the worms' reaction, and the more the production of cutaneous excretions becomes abundant. There is a tendency to progressively neutralize the reaction solutions. The regular spraying of the worms with the same reaction solutions made it possible to observe that the more the $\mathrm{pH}$ deviates from neutral, the greater the variation in the weight of the live worms. This drop in the fresh weight of the worms is indicative of the release of cutaneous excretion under the effect of $\mathrm{pH}$. The neutralizing power is all the more important as the fresh weight of the worms is substantial. The application of cutaneous excretions alone obtained by stimulation with petroleum ether causes the neutralization of the acidic and basic reaction solutions. Given these results, it can be said that in nature, Lumbricus terrestris would directly affect the $\mathrm{pH}$ of the soil through its skin excretion. The $\mathrm{pH}$ factor is a determining factor in soil productivity and the distribution of plant groups. Conditions of high soil acidity or alkalinity significantly affect plant growth because of the effect of $\mathrm{pH}$ on the solubility of mineral elements and their uptake by the root system. In fact, the majority of cultivated plants grow in soils with a $\mathrm{pH}$ close to 
neutrality. The neutralizing effect of cutaneous excretion of Lumbricus terrestris would explain the positive role that earthworms play in plant production. Keywords: Lumbricus terrestris, Cutaneous excretion, $\mathrm{pH}$, Neutralizing effect.

O4. Valorization of Essential Oils of Oregano and Eucalyptus in vitro and in vivo Biological Control of Fire Blight caused by Erwinia amylovora

Afaf Ameur ${ }^{1,2^{*}}$, Naima Rhallabi ${ }^{2}$, Houda Benchnikh ${ }^{1,3}$, Marie Epiphane Doussomo 1,3, Abdellatif Benbouaz$\mathrm{za}^{1}$, My Mustapha Ennaji ${ }^{2}$, and El Hassan Achbani ${ }^{1 \S}$

1 Laboratory of Bacteriology and Biocontrol, Plant Protection Unit- INRA-Meknes Morocco; ${ }^{2}$ Laboratory of Virology, Microbiology, Hygiene and Bioactive Molecules and Quality/Eco-Toxicology and Biodiversity, Faculty of Sciences and Techniques, Hassan II, Mohammedia, University Mohammedia, Morocco; ${ }^{3}$ Departement of Biology, Faculty of Sciences, Kenitra, Morocco. *Presenting Author: afaf.ameur@gmail.com. §Corresponding Author: achbani105@gmail.com.

\section{Abstract}

Fire blight is a disease caused by Erwinia amylovora, which affects several plant species, mainly belonging to the rosaceae family and ornamental maloids. In Morocco, fire blight made its first appearance in 2006 in the region of Meknes. Since then, it has led to significant economic losses and in the absence of effective control of this disease, research has now been turning to biological control. The present work aims to evaluate the effectiveness of different essential oils in vitro and in vivo against E. amylovora. Essential oils are extracted from aromatic and medicinal plants using the hydrodistillation technique. The minimum inhibitory concentrations were determined by the dilution method in the liquid medium. And also Tests were performed also in vivo on immature fruit of pear and in the field against the bacterium E.amylovora. For the in vitro test, most essential oils show strong antibacterial activity at very low concentrations. The choice is focused on oregano and eucalyptus, in particular, exert an antibacterial effect against the pathogen; they inhibit the development of E. amylovora with inhibition percentages respectively of $78.37 \%$ and $63.27 \%$. The MIC and MBC of the oils vary between
32 and $10 \mu \mathrm{g} / \mathrm{ml}$ and the $M B C / M I C$ ratio shows that both oils are bacteriolytic. For the in vivo test, the oils have been shown to be remarkably effective on immature pear fruit and at the field level. Keywords: Erwinia amylovora, rosaceae, biological control, essential oils, oregano, eucalyptus.

\section{O5. Influence of Application of Silicon on Black Cumin (Nigella Sativa L.) Cultivation in Salt stressed environ- ments}

Jamila Fahimi ${ }^{1,2^{*}}$, Fouad Achemchem', Zakia Bouzoubaâ ${ }^{2}$, Nabil Saffaj', Rachid Bouharroud ${ }^{2}$, and Rachid Mamouni'. ' Team of Materials, Catalysis and Natural Resources Development, Department of Chemistry, University lbn Zohr, FSA. Agadir; 2 Agrophysiology \& Post Harvest Laboratory Natural Resources and Local Product Research Unity, CRRA-Agadir, INRA, Morocco. "Corresponding and Presenting Author: jamila. fahimi@edu.viz.ac.ma /f.achemchem@uiz.ac.ma.

\section{Abstract}

Salinity is a harmful abiotic factor to agricultural production particularly in arid and semi-arid regions. In salt conditions almost every physiological and biochemical pathway in the plants are affected. The application of silicon is considered as an alternative approach to mitigate salt stress in plants. For that, this research aims to study the influence of silicon on Black Cumin Nigella sativa (L) cultivation grown under three concentrations of salinity $(0,50$ and $100 \mathrm{mM})$. The Results showed a significant decrease of photosynthetic pigments contents, the ratio $\mathrm{K} / \mathrm{Na}$ and biomass in salt conditions. While, the addition of silicon acted positively in Nigella sativa (L). Keywords: Silicon; Nigella sativa (L); Salinity; Tolerance.

\section{O6. Breeding Effect on Grain Yield and Protein Content of Six Moroccan Durum Wheat Varieties Released Dur- ing the Last Three Decades}

Abdelali Boussakouran ${ }^{1 *}$, El Hassan Sakar ${ }^{1}$, Mohamed El Yamani ${ }^{1}$, Mouna Taghouti ${ }^{2}$, and Yahia Rharrabti ${ }^{1}$

1 Laboratory of Natural Resources and Environment, Polydisciplinary Faculty of Taza, Unviersity Sidi Mohamed Ben Abdellah, Taza, Morocco; ${ }^{2}$ National Agricultural Research Institute (INRA), BP 6356, Rabat, 
Morocco. "Corresponding and Presenting Author: boussakouran@yahoo.fr.

\section{Abstract}

Durum wheat (Triticum turgidum L. var. durum) is an important crop in Mediterranean environments where yield potential is usually constrained by drought stress and requires efforts towards the release of new varieties not only more productive, but also offering improved yield stability across a range of environmental conditions. The present work aims to explore breeding effects during the last three decades on grain yield and protein content of six Moroccan durum wheat varieties grouped in old, intermediate and modern and subjected to two contrasting environments (irrigated and rainfed) during 2016-17 crop season. Measurements consisted in the following parameters: grain yield (GY) and its components spikes per square meter (SM2), grain per spike (GS), and thousand grain weight(TGW); protein content (PC); yield components were determined as: plant height (HP) and harvest index (HI). Analyses of variance demonstrated the predominance of water regime effect on the majority of investigated traits since it explained more than $77 \%$ of their total variance. Breeding effects on GY was marked by a genetic gain of 179 kg.ha-1.yr-1 under favorable conditions but a significant loss of 66 kg.ha-1.yr-1 under stressed conditions. Old varieties displayed a relative yield stability across environments compared to modern ones which had a bad performance under water shortage conditions. On the contrary, PC showed a significant genetic gain under both irrigated and rainfed condition $(0.084$ $\% . y r-1$ and $0.096 \% . y r-1$, respectively). Under rainfed conditions, correlation analyses revealed that GY was positively correlated to SM2 ( $\left.r=0.952^{* *}\right)$, $\mathrm{PH}\left(r=0.829^{*}\right)$ and TGW, $\left(r=0.934^{* *}\right)$. However, a negative association was shown between $G Y$ and PC ( $r=-0.897 *)$. Regarding irrigated treatment, GY was highly dependent on its main components SM2 $\left(r=0.935^{* *}\right)$ and positively correlated to PC ( $r=$ $0.901 *)$, however $G Y$ was negatively associated to $\mathrm{PH}(\mathrm{r}=-0.871 *)$ and TGW $(r=-0.960 * *)$. Relationship between GY and PC was of a curious pattern. In fact, while under favorable conditions increases in $G Y$ were accompanied by higher levels of PC, the opposite was observed under stressed conditions where higher GY was obtained in detriment of good grain quality (lower PC). Keywords: durum wheat, grain yield, protein content, breeding effects, Morocco.

\section{Session IX: US-Morocco Research \& Higher Education Collaboration}

\author{
Co-Chair: Khalid Meksem, Southern Illinois Uni- \\ versity, USA \\ Co-Chair: Ahmed Bouaziz, IAV, Morocco
}

\section{O1. The Moroccan American Commission for Education- al \& Cultural Exchange Mission. The Fulbright Mission in Morocco.}

James Miller.

The Moroccan American Commission for Educational \& Cultural Exchange (MACECE), Rabat, Morocco.

\section{Abstract}

Not Provided. O2. Application Process to the Graduate School at
Southern Illinois University, Carbondale, IL, USA

\section{Oumaima Chetto}

IAV Hassan II, Rabat, Morocco. Propsective Graduate Student: Department of Plant, Soil, and Agricultural Sciences, College of Agricultural Sciences, Southern Illinois University, USA.

\section{Abstract}

Not provided.

O3. Meeting Challenges and Demands of Agriculture through Research, Education and Outreach and a Need for More Young People

\section{Karen Stoelzle Midden*}

College of Agricultural Sciences, Southern Illinois University, Carbondale, Illinois, USA. "Presenting and corresponding Author: kmidden@siu.edu. 


\section{Abstract}

There is a new paradigm for professional and scholarly demands in agriculture yet much of the public, especially students, fail to be aware of the critical need and exciting opportunities in these expanding areas. Today's global agriculture encompasses traditional farming and now also includes much broader practices and challenges. The need to address growing global population, urbanization, climate change and other world developments has inspired scientist, humanitarians, planners and leaders to seek agriculture practices to serve our changing society. These practices include alternative food systems and securities, urban agriculture, new seed development, food engineering, conservation of natural resources, recreation, and other areas connected to economics, politics, social, cultural, environmental, mental and physical human health; each that demand attention in education and research. To meet the demands and challenges, it is critical to engage talented students to pursue careers in research, education and industry for the future of agriculture. Data from the United States Department of Agriculture states that, in the US alone, an average of nearly 58,000 high-skilled agriculture and related job openings are expected annually with only 35,000 graduates qualified to fill those positions. (https://www.purdue.edu/usda/employment/). There is a need for more young people to pursue their education, research and careers in agriculture. This presentation will discuss today's agriculture as it relates to educational opportunities to prepare students for meaningful careers in agriculture, agriculture programs at Southern Illinois University and potential collaboration between Southern Illinois University and Moroccan universities to serve the global agriculture community. Keywords: agriculture research, careers in agriculture, graduate education, employability, global agriculture.

\section{O4. Advice to Graduate Students}

\section{Khalid Meksem ${ }^{*}$}

Southern Illinois University, USA. "Presenting and corresponding Author: meksem@siu.edu.

\section{Abstract}

Not provided.

\section{Concurrent Session X: Plant Pathol- ogy, Nematology, and Disease Resistance}

\section{Co-Chair: Lamia Ghaouti, IAV, Morocco Co-Chair: Mustapha El Bouhssini, ICARDA, Ra- bat, Morocco}

\section{Current Status of the Root-Knot Nematodes (Meloidogyne spp.) Attacking Vegetable Crops in the Souss Region of Morocco}

Fouad Mokrini ${ }^{1 *}$, Soukaina Janati ${ }^{2}$, Abdellah Houari ${ }^{2}$, Aicha El Aissami ${ }^{3}$, Ahmeh Wifaya', Abdelaziz Mimouni $^{1}$, and Mohamed Sbaghi ${ }^{4}$

1 Integrated Crop Protection Research Unit, Nematology Laboratory, INRA, Agadir, Morocco; ${ }^{2}$ Faculté Polydisciplinaire de Taroudant, Laboratoire des Biotechnologies, Valorisation et Environnement, University lbn Zohr, Taroudant, Morocco ; ${ }^{3}$ Faculté des Sciences, Université Mohammed V, Rabat, Morocco; ${ }^{4}$ National Institute of Agronomic Research, Scientific Division, Rabat, Morocco. "Corresponding and Presenting Author: fmokrini.inra@gmail.com.

\begin{abstract}
Root-knot nematodes (Meloidogyne spp. ; RKN) are the most common nematodes attacking vegetable crops and causing serious damage worldwide. Therefore, we aimed to review the current status and impacts of this nematode on vegetable crops in the Saiss region of Morocco. The earliest report of nematodes attacking vegetable crops in Morocco was in 1982, by Janati, who recorded the presence of four species of Meloidogyne, $M$. javanica, $M$. incognita, $M$. arenaria and $M$. hapla. Since then and up to now several surveys of nematodes associated with vegetable crops were conducted and revealed the presence of four species. However these surveys were limited and primitive since not all vegetable crops production areas were sampled to detect RKN. An intensive survey of RKN was conducted on vegetable crops growing
\end{abstract}


regions of Sous-Massa and showed that the root-kont nematodes (RKN) were detected in seventy-five out of 87 localities sampled (=86\%). Four species were detected, viz. $M$. javanica, $M$. incognita, $M$. arenaria and $M$. hapla. Both $M$. javanica, $M$. incognita were the most dominant species. The highest density of $\mathrm{J} 2$ of Meloidogyne spp. was recorded in Khmit Ait Amira with 349 J2 (Meloidogyne spp.) (100 g soil)- 1. In view of the estimates of nematode densities obtained in this survey, once can assume that this genus damage vegetable crops in many cases. Field studies on the population dynamics and the damage function are necessary to estimate the economic impact of this nematode on vegetable crops in the Souss region. The current status of RKN on vegetable crops in the Souss region is advanced and much work is needed on the distribution, damage threshold and on the management issues. Keywords : Root-kont nematodes, Vegetables crops, Survey, Souss-Massa.

O2. A New Source of Resistance to Hessian Fly (Mayetiola destructor (Say)) in Durum Wheat (Triticum durum Desf.)

Hajar Brahmi ${ }^{1,2^{*}}$, M. El Bouhssini ${ }^{2}$, F. Bassi ${ }^{2}$, S. El Haloui ${ }^{3}$, A. Sabraoui ${ }^{2}$, K. El Fakhouri ${ }^{2}$, and A. Lazraq ${ }^{1}$

1 Laboratory of Functional Ecology and Environment, Department of Biology, Faculty of Science and Technology, Fez, Morocco; ${ }^{2}$ International Center for Agricultural Research in the Dry Areas (ICARDA), Rabat, Morocco; ${ }^{3}$ National Institute of Agronomic Research, Regional Center of Settat, INRA, CRRA, Settat, Morocco. *Presenting and corresponding Author: hajar. brahmi01@gmail.com.

\section{Abstract}

Durum wheat (Triticum durum Desf.) is a major component of human diet worldwide, and is grown over 1 million ha in Morocco. However, this crop suffers from numerous pests, in particular Hessian fly (Mayetiola destructor (Say)) causing serious economic damage. The use of resistance cultivars has been the economic and environmentally friendly approach to protect wheat from pest damage. Several resistant varieties to Hessian fly have been developed and registered in the Moroccan catalogue. However, because of the development of virulent biotypes, there is a need for continuous search of new sources of resistance. The present study was carried out under controlled conditions at INRA in Settat for two years (Spring 2015 -2016) to screen a collection of 380 genotypes of durum wheat. Promising genotypes from the initial screening were re-evaluated for confirmation in replicated trials. The results of phenotypic identification revealed one source of resistance to this pest. Genetic studies of this new source will be undertaken to determine if this genotype carries a new gene for resistance to Hessian fly in Morocco. Keywords: Durum wheat, Hessian fly, resistance, screening.

\section{O3. Morphological, Pathogenic and Molecular Char- acterization of Rhizoctonia solani Strains Isolated from Potato Tubers}

Imad Kotba ${ }^{1,2,3^{*}}$, Mohammed Achouri ${ }^{1}$, Amina Ouazzani Touhami ${ }^{2}$, Allal Douira ${ }^{2}$, and El Hassan Achbani ${ }^{3}$

${ }^{1}$ IAV, Complexe Horticole, Agadir, Morocco; ${ }^{2}$ Laboratoire de Botanique, Biotechnologie et de Protection des Plantes, Faculté des Sciences, Université Ibn Tofail, Kenitra, Morocco; ${ }^{3}$ Laboratoire de Phytobacteriologie et de Lutte Biologique du CRRA de I'INRA, Meknès, Morocco. *Corresponding and Presenting Author: imad.kotba@gmail.com.

\section{Abstract}

Rhizoctonia solani Kühn [teleomorph: Thanatephorus cucumeris (Frank) Donk.] is an important fungal pathogen widespread in all potato growing areas of the world that causes stem canker and black scurf of potato (Solanum tuberosum L.). The aim of this study was to find a simple and reliable technique for determining the pathogenicity of Rhizoctonia solani isolates. For this purpose, sixty isolates of R. solani obtained from sclerotia on potato tubers, collected from different markets of Agadir and Casablanca regions, were studied for their morphology, pathogenicity and molecular characteristics. They were morphologically characterized by the production of sclerotia and moniloïd cells, and by the mycelium growth capacity at $15,20,25,30$ and $35^{\circ} \mathrm{C}$. This morphological characterization leads to three groups of isolates. The first group contained $\mathrm{PO} 1$ and $\mathrm{PO} 3$ isolates, which were able to develop under $35^{\circ} \mathrm{C}$. However, under $25^{\circ} \mathrm{C}$, they didn't develop sclerotia. The second group, only 
formed by L17.1 isolate, did not form sclerotia under $25^{\circ} \mathrm{C}$ and was not able to develop under $35^{\circ} \mathrm{C}$. The third group, formed by several isolates, developed sclerotia under $25^{\circ} \mathrm{C}$ conditions and were not able to grow under $35^{\circ} \mathrm{C}$. Also, a positive correlation was consistent between the production of sclerotia and moniloïd cells formation. The anastomosis reaction revealed that PO1, P03, L17.1, and L4.1 isolates were identified as AG-4 and AG-3 for the other isolates. The pathogenic characterization has shown that P01, P03, L4.1, and L17.1 isolates caused important damping off of radish, tomato, beans, zucchini, and melon. However, the other isolates showed only a minor damping off disease rate. The molecular characterization confirmed the classical anastomosis grouping of the isolates into AG-3 and AG-4 anastomosis groups. The molecular characterization is the most rapid and reliable technique to determine the anastomosis group of unknown isolates. The three tests including the pathogenicity, the cultural anastomosis grouping, and the molecular method helped separating the studied isolates to two groups. Keywords: Rhizoctonia solani, Potato, Anastomosis, Morphology, Pathogenicity, Molecular characteristics.

O4. Characterization and Diversity of Plant-Parasitic Nematode Communities Associated with Saffron (Crocus sativus L.) in Taliouine-Taznakht (Morocco) and their Relationships with Some Soil Physicochemical Characteristics

Hinde Benjlili, ${ }^{1 *}$, K. Elkassemi' ${ }^{1}$, Z. Ferii', E. Mayad ${ }^{2}$, and K. Cherifi ${ }^{2}$

' IAV Hassan II, Campus d'Agadir, Département de Protection des Plantes, BP 18, Agadir, Morocco ; ${ }^{2}$ Université Ibn Zohr, Faculté des Sciences, Département de Biologie, Laboratoire Biotechnologie et Valorisation des Ressources Naturelles, BP 8106 , Agadir, Morocco. "Corresponding and Presenting Author: hinde.benjlil@gmail.com.

\section{Abstract}

Plant-parasitic nematodes (PPN) are the most destructive group of plant pathogens in the world, and they are an economically important group of soil pathogens. Moreover, they cause $12.3 \%$ losses to agricultural products worldwide. Saffron (Crocus sativus L.) considered as the most expensive spice in the globe; it has been grown in Morocco for centuries in the region of Taliouine-Taznakht. This latter, has a great saffron reputation nationally and internationally. The Saffron's crop receives increased attention and cultivation according to Morocco's green plan. As this situation may increases Saffron crop sensitivity to many pathogens as well as plant parasitic nematodes (PPN). In order to manage this bioagressors a study of their biodiversity is necessary. In this context, nematode communities were characterized in 163 soil samples collected from 11 rural communes in different altitude. Fifteen genera were identified belonging to 12 families; four genera of PPN considered to be harmful for saffron were detected: Ditylenchus, Helicotylenchus, Pratylenchus, and Aphelenchoides. Their frequencies are respectively $92 \%$; $31 \% ; 63 \%$, and $43 \%$ in Taliouine; while in Taznakht, $92 \% ; 21 \% ; 64 \%$, and $56 \%$. Regarding assessing the diversity of (PPN) associated with saffron in all sites, the genus richness $(G)$ ranged from 3 to 9 whereas Shannon diversity index $\left(\mathrm{H}^{\prime}\right)$ varies from 0,9 to 1,47 and Evenness (E) tends to 1. The relationship between plant-parasitic nematodes and the soil physicochemical properties were investigated with principal component analysis and co-inertia analysis. Keywords: Frequency; diversity; plant parasitic nematodes; saffron; Taliouine Taznakht.

\section{O5. Evaluation of Diverse Sets of Barley Germplasm for Resistance to Scald Disease (Rhynchosporium secalis)}

Houda Hiddar ${ }^{1,2^{*}}$, Sajid Rehman², Zakaria Kehel ${ }^{2}$, Athanasios Tsivelikas ${ }^{2}$, Ramesh P.S. Verma ${ }^{2}$, Abdelkarim Filali-Maltouf ${ }^{1}$, and Ahmed $\mathrm{Amri}^{2}$

1 Microbiology and Molecular Biology Laboratory, Department of Biology, Faculty of Sciences, Mohammed $V$ university, Rabat, Morocco; ${ }^{2}$ International Center for Agricultural Research in the Dry Areas (ICARDA), Rabat, Morocco. "Corresponding and Presenting Author: hiddar.houda@gmail.com.

\section{Abstract}

Leaf Blotch, also known as scald, is one of the most destructive diseases of barley (Hordeum vulgare L.); caused by the haploid imperfect fungus Rhynchosporium secalis. Scald is widely distributed throughout the 
world and can cause $40 \%$ reduction in grain yield. The use of resistant cultivars remains to be the most effective, economical and environmental friendly way to control the disease but the genetic resistance to Moroccan isolates is poorly understood and only few sources of resistance have been identified. This study aims at the identification of new and effective sources of resistance to scald within various subsets: Best bet subset selected using the Focused Identification of Germplasm Strategy (FIGS; 80 accessions), reference set identified within the Generation Challenge Program (GCP; 204 accessions), and an association mapping panel (284 accessions) developed by ICARDA breeders. All the accessions were sown and evaluated at seedling stage under controlled conditions. We have found that of the 568 barley accessions tested, 78 barley accessions (14\%) were found to be resistant, 86 accessions ( $15 \%$ ) to be moderately resistant, 104 accessions (18\%) to be susceptible, and 103 accessions (18\%) to be highly susceptible, respectively. Most of the resistant accessions (125; $22 \%$ ) showed immune reaction. These new sources of resistance will be a useful resource for resistance barley breeding programs around the world. Keywords: Barley, Scald, Rhynchosporium secalis, resistance.

\section{O6. Diversity and Distribution of Entomopathogenic Nematodes in Morocco}

Youssef Ben Seddik ${ }^{1,2^{*}}$, Abdelali Blenzar ${ }^{1}$, Abdelmalek Boutaleb Joutei ${ }^{2}$, and Said Amiri ${ }^{2}$

1 College of Sciences, University of Moulay Ismaiil, Meknes, Morocco; ${ }^{2}$ National School of Agriculture, Meknes, Morocco. "Corresponding and Presenting Author: benseddikyoussef@gmail.com.

\section{Abstract}

A survey was carried out in three Moroccan regions: Saiis, Middle Atlas and Tafilalet. The aim of the survey was the selection of Moroccan isolates of Entomopathogenic Nematodes (EPN) for their use in biological control against soil-dwelling pests. 169 soil samples were tested for the presence of EPN by Galleria baiting technique. 14 EPN isolates were found in 13 soil samples ( 2 isolates were found in the same sample): 8 isolates are Heterorhabditidae nematodes and 6 isolates are Steinernematidae nematodes. Soils where EPN were found differ as regards some characters (texture, $\mathrm{pH}$ and organic matter content). Keywords: Entomopathogenic nematodes, Galleria baiting technique, Heterorhabditidae, Steinernematidae.

\section{Concurrent Session XI: Walter Man- agement \& Uses \\ Resistance}

\section{Co-Chair: Abdelaziz Mimouni, INRA, Agadir, Morocco \\ Co-Chair: Meryem El Mrini, IAV, Rabat, Morocco}

\section{O1. The Public-Private Partnership in Irrigation: What is the Impact on the Technical Efficiency of Citrus Farms in the Souss-Massa Region?}

Nassreddine Maatala ${ }^{1^{*}}$, Aziz Fadlaoui ${ }^{2}$, Philippe Lebailly ${ }^{3}$, and Majid Benabdellah ${ }^{4}$

${ }^{1}$ Hassan II Agronomy and Veterinary Institute, Rabat, Morocco; ${ }^{2}$ INRA, Regional Agricultural Research Center of Meknes, P.O. Box 578, Meknes, Morocco; ${ }^{3}$ Department of Economics and rural sociology, Gembloux Agro-Bio-Tech, University of Liege, Belgium; ${ }^{4}$ Department of Social Sciences, Hassan II Agronomy and Veterinary Institute, Rabat, Morocco. "Corresponding and Presenting Author: m.nassreddine@gmail.com.

\section{Abstract}

The Public-Private Partnership (PPP) is a cooperative arrangement that allows the Government to entrust to a private company some public facilities or incorporeal investments from conception to management for a specific period. In the irrigation sector, this type of partnership appeared in 2005 and concerned the El Guerdane project in the Souss-Massa area. Since then, the Government has continued to implement other projects all over the country. However, the existing literature consulted allowed us to identify a significant deficit in the evaluation and the impact of such projects on farm performance as well as the use of water resources. The aim of this research is to evaluate the impact of two irrigation modes related to water service management on the technical perfor- 
mance of citrus farms, which are in our case the PPP mode adopted in the El Guerdane project and the conventional mode whose management is entrusted to a public institution. The selected sample includes 120 farms located in the two perimeters of El Guerdane and Issen. Apart from the fact that Issen perimeter is managed by the Regional Office for Agricultural Development of Souss-Mass, the two perimeters have similar pedo-climatic conditions of production mainly because of their proximity. In addition, the citrus growers in both areas adopt the same production technology. The farms in our sample were selected using the propensity score matching method, and the estimation of efficiency scores was performed using the parametric stochastic frontier approach to the production by the Frontier 4.1 software. The results revealed that technical efficiency scores range from $16 \%$ to $92 \%$ for all farms selected in our sample. The average technical efficiency is $68 \%$. Basically, this result demonstrates that there is still room for increased production without additional input from factors of production including irrigation water. Furthermore, the determinants analysis shows that El Guerdane's farms are relatively more technically efficient than Issen's farms. Also, It has been found that membership of professional organizations contributes to the improvement of technical efficiency. In view of these results, it is recommended that the agricultural council structures intensify their support by targeting the least efficient farms. Keywords: Public-Private Partnership, Irrigation, Technical efficiency, El Guerdane project.

O2. Plant Water Status Changes Induced by Water Deficit during Phenological Growth Stages of Young Olive Trees (Olea Europaea L.) Grown in Northern Morocco

Mohamed El Yamani ${ }^{*}$, El Hassan Sakar, Abdelali Boussakouran, and Yahia Rharrabti. Laboratory of Natural Resources and Environment, Polydisciplinary Faculty of Taza, B.P 1223, Taza-Gare, University Sidi Mohamed Ben Abdellah, Taza, Morocco. "Corresponding and Presenting Author: elyamani.med90@ gmail.com and mohammed.elyamani@usmba.ac.ma.

\section{Abstract}

The present work investigates plant water status changes of young olive trees (Olea europaea L.) belonging to three Moroccan varieties (Moroccan Picholine, Menara and Haouzia) grown under three water regimes namely T100 (unstressed), T50 (medium stress) and TO (high stress) in northern Morocco (Taza Province). Leaf relative water content (LRWC), water potential $\left(\Psi_{w}\right)$, and stomatal conductance (gs), were measured during the principal growth stages; dormancy ( $B B C H$ code: 00 ), leaf development (Code: 15), flowering (Code: 65), fruit development (Code: 71), and fruit maturity (Code: 81) for the growing season 2015-2016. ANOVA analyses showed that the three traits examined were mainly influenced by the water regime and the phenological growth stage; LRWC and $\Psi_{w}$ were under the effect of both factors in equal proportion, while gs was mostly affected by the water regime. Impacts of variety and interactions were of a very minor magnitude. Water deficit reduced LRWC by an amount of $12 \%$ in high stressed olive plants, while $\Psi \mathrm{w}$ and gs were more affected with a reduction of $34 \%$. The maximum reduction was shown during fruit development. Between phenological growth stages, the highest values of the three traits were recorded during dormant stage, whilst the lowest values were observed at fruit development stage. Among varieties, no significant differences were shown for gs, whereas Menara and Moroccan Picholine displayed the lowest values for LRWC and $\Psi_{w^{\prime}}$ respectively. Relationships among water status traits highlighted a close positive correlations between them $\left(r>0.90^{* * *}\right)$. Stage-to-stage fluctuations in rainfall and temperatures and water needs, and adaptive mechanism of olive trees under water deficit could explain the findings. Keywords: Olea Europaea L., growth stage, water deficit, water status.

O3. Effects of Water Regime and Organic Amendment on Flowering Capacity, Growth and Development of Replacement Corms of Saffron Under Different Planting Densities

Youssef $\mathrm{Karra}^{1 *}$, Abdelghani Tahiri 1, Ahmed Wifayal, Mohammed Amine Serghini ${ }^{2}$, and Abdelaziz Mimouni ${ }^{1}$

1 Research Unit "Natural Resources and Local Products", INRA, CRRA, Agadir, Morocco; ${ }^{2}$ Biotechnology and Natural Resources Valorization Lab, Department of Biology, Faculty of Sciences, Ibn Zohr University, 
Agadir, Morocco. ${ }^{*}$ Corresponding and Presenting Author: youssef.karra@gmail.com.

\section{Abstract}

In Morocco, saffron cultivation is mainly located in Taliovine region $(95 \%)$, it's a mountainous area $(>1500 \mathrm{~m}$ of altitude) characterized by a semi-arid climate. Saffron is organically grown where local corms are multiplied to serve as a propagation material. The number and size of corms are main parameters for saffron yield expression which is affected by water supply, replacement corms intensity and organic amendment rates. The objective of this study is to study the effect of planting density, irrigation and compost amendment on growth and yield of saffron. Corms were planted according to four levels of planting densities $(25,50,100$ and 140 corms.m-2) in two trials plot. In the first plot, different water regimes were allocated to different percentages of evapotranspiration reference $(100 \%, 75 \%, 50 \%$ and $30 \%$ of ETo). In the second plot, compost was amended twice at different rates. The results obtained showed that the irrigation regime at $75 \%$ ETo allowed a significant efficiency on corm and stigma yields, the high planting densities revealed a positive effect on number of corms and shoots, although, low densities showed interesting results on corms multiplication rate.On the other hand, the compost amendment had a positive effect on corm development by promoting their multiplication, to the extent that the second input was benefic for the development phase of replacement corms. Finally, the choice of the optimum planting density and the appropriate irrigation and amendment rate is extremely crucial to ensure good growth and a significant yield of saffron as a perennial crop. Keywords: Saffron of Taliouine, planting density, corms multiplication, water regime, organic amendment.

\section{O4. Adaptive Strategies in Date Palm Revealed by Con- focal Imaging Technologies}

Ting Ting Xiao* ${ }^{*}$ and Ikram Blilou ${ }^{\S}$

Plant Cell and Developmental Biology, Biological and Environmental Science and Engineering, King Abdullah University of Science and Technology, Thuwal 23955-6900, Saudi Arabia. "Presenting Author: tingting.xiao@kaust.edu.sa. §Corresponding Author: Ikram.blilou@kaust.edu.sa.

\section{Abstract}

Date palm are confronted by harsh environmental conditions and have therefore adapted various strategies to survive the hostile environment. To unravel the underlying mechanisms of adaptation to desert conditions we conducted a detailed analysis of date palm tissue anatomy at different developmental stages. Using confocal imaging we reveal new anatomical features and complex structures in roots, shoot and leaves explaining strategies of adaptation of date palm to desert conditions.

\section{O5. Evaluation of spatio-temporal variability of Runoff and Sediment Yield for a Nakhla Watershed Using SWAT Model}

Roukia Boukhari Taleb ${ }^{1 *}$, Mustapha Naimi ${ }^{1}$, Mohamed Chikhaoui', Damien Raclot ${ }^{1,3}$, Mohammed Sabir ${ }^{2}$. 1 IAV Hassan II, Rabat, Morocco; ${ }^{2}$ Ecole Nationale Forestière d'Ingénieurs, Tabriquet, Salé, Morocco; ${ }^{3}$ Institut de Recherche pour le Développement, UMR LISAH, Montpellier, France. "Corresponding and Presenting Author: rokia.boukhari.taleb@gmail.com.

\section{Abstract}

Deterioration and degradation are two main factors caused by the increase and intense use of natural resources, soil, water as well as the aggressive natural context that affects both quality and quantity. To solve this problem, evaluating the states and evolution over time and space are essential for a planned and a sustainable future to soil and water management. SWAT 2012 (Soil and Water Assessment Tool) model was thus implemented on a daily time frame for the Nakhla watershed, which is located at 20 kilometers in the south of the city of Tetouan. Treatment of environmental data (MNT, pedology, satellite imagery and climatic data) has allowed us to describe the natural characteristic of the field at a resolution adapted to the objectives of the study. The adopted methodology was composed of the combined use of the SWAT agro hydrological model implemented in the QGIS geographical information system open source for op- 
erations of analysis and modeling of hydrological processes at the level of the watershed area and of SWAT-CUP tool to optimize the operations of sensitivity and uncertainty analysis. The calibration process was performed on two temporal scales; monthly and daily; and on the two stations Timezouk and Nakhla; and over three periods: warm-up period from 1980 to 1983 , calibration period from 1984 to 1990 , and the validation period from 1991 to 1994 . The results acquired after calibration (NS $=0.8, \mathrm{R}^{2}=0.8$ ) and validation (NS $=0.7, R^{2}=0.8$ ) at monthly timescale were adequate and presented good level of realism in the representation of the interactions between the different compartments of the model; as well as the simulation of the flow rates; and also in assessing the spatial distribution of the importance of erosion across the basin and quantifying sediment yield. Keywords: QGIS, SWAT, Hydrology, Sediment, Nakhla, Morocco. 
POSTER PRESENTATIONS ABSTRACTS

WEDNESDAY \& THURSDAY

MAY 9 \& 10, 2018

P001. In vitro Germination of Orange Tree's (Citrus sinensis) Immature Embryos

Karim Mahmoudi ${ }^{1,2}$, Najat Handaii', Mohammed Ibriz $^{2}$, Najat Arsalane', Tarik Aderdouri ${ }^{1,2}$, Kawtar Label $^{1,2}$, and Hamid Benyahya ${ }^{1}$

1 Laboratoire d'Amélioration ef Conservation des Ressources Phytogénétiques, Institut National de la Recherche Agronomique, Kenitra, BP 257, Morocco; ${ }^{2}$ Laboratoire de Génétique et Biométrie, Université Ibn Tofail, Faculté des sciences Kenitra. Morocco. *Corresponding and Presenting Author: karim 1.mahmoudi1@gmail.com.

\section{Abstract}

Oranges constitute the major part of the production of citrus fruit, which is the most important fruit group in international trade. The creation of new triploid hybrids via the rescue of immature embryos allows diversification of the varietal profile of orange trees. The objective of this study Is to optimize the in vitro germination of immature embryos according to the chemical composition of four in vitro culture media in two variétise of orange trees (Pinéale and Parsons Brown). At the maturity stage, the fruit was harvested and the extracted seeds were classified according to their size. Only small or flat seeds were cultured in a base medium of Murashig and Tuker (MT) under sterile conditions. The different growth regulator concentrations were tested to obtain the best medium for seedling development: M1 (MT + $1 \mathrm{mg} /$ I GA3), M2 $(\mathrm{MT}+1 \mathrm{mg} / \mathrm{I}$ Kenitin $+0.5 \mathrm{mg} / \mathrm{I} \mathrm{BAP}+0.1 \mathrm{mg} /$ I ANA), M3 (MT + $25 \mathrm{mg} /$ I adenine sulphate), M4 (MT + $0.5 \mathrm{mg} / \mathrm{I}$ Kenitin + $0.5 \mathrm{mg} / \mathrm{I} \mathrm{BAP}+1 \mathrm{mg} /$ I GA3). For bot Orange variétise Pinéale and Parsons Brown, the germination rate Is maximum in $M 3$ medium respective Ly at percentages of $100 \%$ and $90 \%$. variétise Be tween 6 and 7 dayas. With respect to growth rate ( $\mathrm{mm} /$ week), bot variétise knew a variation in the four media. Similarly, the maximum acclimation rate in the $M 1$ medium Is $80 \%$ and $90 \%$ respec-

tive Ly for the Pinéale and Parsons Brown variétise. In general, the smaller the embryos, the more sensitive they are to the composition of the culture medium. It Is therefore essential to optimize the components of the medium to promote their growth and their in vitro developments. Therefore, the medium Ml (MT + $1 \mathrm{mg}$ / I GA3) remains the best to promote good germination in short time and a better acclimatizationrate. Keywords: Citrus, Orange tree, triploidy, in vitro germination, immature embryos.

\section{P002. The Potential of Cactus Pear as Food and Forage Crop for Sustainable Agriculture in the Arid and Semi- Arid Regions}

\section{Mohamed Arba*}

Plant Ecophysiology and Cultures of Arid Zones Laboratory, Department of Horticulture, Hassan II Institute of Agronomy and Veterinary Medicine in Agadir, B.P. 121, Ait Melloul 5615, Morocco. *Presenting author: arbamohamed@yahoo.fr.

\section{Abstract}

This paper describes the importance of cactus pear and cladodes as food and forage crop in the arid and semi-arid regions and its worldwide distribution. The importance of cactus pear in the sustainable agricultural systems of the arid and semi-arid regions has been discussed. The physiological features of cactus pear and the importance of this plant in Morocco are presented. The potential of cactus pear as food crop and the contribution of cactus pear fruits and cladodes in the human nutrition are highlighted. The possibilities of valorization of cactus pear to byproducts, which enable high income for rural populations and the nutritional value of the fruits and young cladodes as food for human are explained. The forage resources of the arid and semi-arid regions and the area of cactus pear used for animal feed in the world are presented. The potential of cactus pear as fodder and the nutrients contents in the pads useful for animals are investigated. The possibilities of mixing pads with other fodders and food intakes developed for ruminants are suggested. The crude proteins contents and minerals in the cactus pear pads have been studied in southern Morocco regarding their requirements by cows and sheep. Keywords: Sustain- 
able agriculture; food crop; animal feed; fodder; human nutrition; animal nutrition.

\section{P003. Olive Growing for Mitigation of Climatic Chang- es Effects}

Oumkeltoum Krimi Bencheqroun'", A. Hadiddou, M. Alghoum, A. Mekkaoui, and L. Hssaini

INRA Meknes, Morocco. "Corresponding and Presenting Author: oumkaltoumkrimi@yahoo.fr.

\section{Abstract}

The olive tree is one of the emblematic species of the Mediterranean where it has been cultivated for more than 6000 years. In fact, 95\% of the world's olive-growing heritage is in the Mediterranean basin. This tree is known for its great hardiness and its high power of adaptation to the severity of natural conditions. Therefore, it is one of the plant species capable of contributing significantly to the mitigation of the effects of climate change and this, thanks to its high tolerance to drought and the high adaptability of its root system to different types soil which gives it its important role in erosion control. Indeed, the olive tree is known among the populations of the Mediterranean as being "THE RICH VEGETABLE OF POOR SOILS". Indeed, the robustness of its root system, its important development and its capacity of adaptation to the physicochemical characteristics of the ground, its structure and its texture increase its capacity of fixation of the soil. No need to mention that the olive tree is characterized by a broadly spreading foliage that allows a clear mitigation of the effects of torrential rains on the ground and facilitates, therefore, the infiltration of runoff water. Similarly, the evergreen leaves (which are usually renewed every three years) which characterize the olive tree and which have fine hairs on their underside, simultaneously allow the preservation of moisture and increase the absorption capacity. carbon in the olive tree. Hence its great role as protector of the environment. Genetically, it is one of the plant species that has great richness and remarkable variability. At present, more than 2000 olive varieties have been identified and are cultivated worldwide. In Spain, 262 varieties have been recorded, 476 varieties in Italy and more than 150 varieties in Algeria. In this plant species, tolerance to drought is a varietal character. Indeed, the level of resistance varies according to the different varieties and according to the pedoclimatic conditions. This is due to the importance of the Genotype X Environment interaction. Hence the interest of comparative trials for the identification of the most successful varieties and best adapted to different climatic conditions. In Morocco, in addition to its ecological role, olive growing is of great socio-economic importance. The latter has always occupied more than half of the national arboreal surface. Recently, the area reserved for olive trees has exceeded 1,000,000 ha. Given the importance of the added value of the olive sector on the national economy, the benefits of products, good quality, this tree (table olives and olive oil) on human health, which explain the rise Demand at the national and international levels, Morocco is consciously committed to the extension of the area reserved for olive growing. This relevant choice will give it a good preservation of the environment and a better mitigation of the effects of climate change on the earth and this thanks to the many characteristics and natural characteristics of the blessed tree including the olive tree. Keywords: Olive tree, climactic changes, natural specificities, characteristics, mitigation, preservation, environment.

\section{P004. Economic Water Management under Climate Change Impact}

\section{Elame Fouad ${ }^{1 *}$, Hayat Liounboui' ${ }^{1}$, Doukkali Rachid ${ }^{2}$}

1 Institut National de la Recherche Agronomique, INRA, Morocco; ${ }^{2}$ Institut Agronomique et Vétérinaire Hassan II, Rabat, Morocco. *Corresponding author :

\section{Abstract}

The recent statistics show a clear downward trend in global and per capita availabilities of water due in particular to climate change. In addition to the recurring droughts that Morocco experienced during the last two decades, this tendency is accentuated by an unceasingly increasing demand, in particular under the effect of the demographic pressure and the economic development. In a context of scarcity and a limitation of potential resources not yet mobilized, Morocco is confronted with the need of changing its supply policy to a demand management one. The 
implementation of such policy requires the adoption of new management instruments and new institutional forms of organization. Accordingly, the present study proposes a tool of modeling and decision-making support which integrates the economic, institutional, hydrological and agronomic aspects. The proposed approach is based on optimization techniques and positive mathematical programming to calibrate an empirical model. Using climate change impact scenarios, this integrated economic model is tested for the basin of Souss-Massa. These simulations include changes in water availability and economic conditions, as well as demand management policy. The study results show that the demand management policy at the river basin level should take into account the regional specificities. The basin's water resources are substitutable and water management policy cannot ignore this aspect and should integrate surface and groundwater resources at the same time. In drought conditions, the water marginal value 'shadow price' increases considerably such that water pricing policy alone cannot result in a rational and a sustainable use of the resource. Keywords: Water resources, climate change, drought, River basin, integrated economic model, scenario.

\section{P005. Monitoring Milk Urea Concentrations as Strate- gies to Improve the Efficiency of Nitrogen use in Haouz Dairy Herds in Central Morocco}

\section{Napoléon Munyaneza ${ }^{*}$ and Youssouf El Hachimi}

Department of Biology, Laboratory of Food, Environment and Health of Cadi Ayyad University of Marrakech.*Corresponding author: munyaneza305@ gmail.com.

\section{Abstract}

The aim of this study was to determine the milk urea concentrations variation and their potential relationships with feeding systems and milk production. A total of 251 bulk milk samples was collected in 83 dairy herds and measured in urea by calorimetric methods at the Laboratory of Food, Environment and Health at Cadi Ayyad University in Marrakech, Morocco. Feeding strategies and milk production data were collected as part of a formal survey and analyzed using Statistical Package for Social Science
(SPSS). The average of urea concentrations obtained was $11,4 \pm 1,4 \mathrm{mg} \mathrm{N} / \mathrm{dl}$, with a range of 8,1 to $15,9 \mathrm{mgN} / \mathrm{dl}$. Out of all samples, $21,8 \% \mathrm{had}$ values outside the reference range (12 $\pm 2 \mathrm{mgN} / \mathrm{dl}), 3$, $1 \%$ had values $>14 \mathrm{mgN} / \mathrm{dl}$ and $18,7 \%$ had values $<10 \mathrm{mgN} / \mathrm{dl}$. The milk production ranged of 4 to 28 liters/day/cow. Data analysis on feeding systems used by farmers revealed that about $12 \%$ used alfalfa alone, $72,2 \%$ alfalfa plus cereals straw, $7,3 \%$ alfalfa and cereals straw plus residues of beet and $8,5 \%$ used alfalfa plus cereals straw supplemented by cereals bran. The correlation between urea concentrations and milk production was observed $(r=$ $0,731 ; p<0,01)$. When yield milk was $>13,5$ liters /cow (51,3\%), milk urea concentrations was $>$ $12 \mathrm{mgN} / \mathrm{dl}$. The milk production was lower and urea in milk less to $10 \mathrm{mgN} / \mathrm{dl}(20 \%)$ when the cows received alfalfa alone or associated to cereals straw. The results showed a large variations of urea milk concentrations in relation with yield milk and feeding systems between farmers. Thus, monitoring urea concentrations in milk would provide information that could help farmers to use nitrogen inputs and increase the profitability under the specific conditions of each farm. Keywords. Urea milk, dairy herds, nitrogen efficiency, milk production.

\section{P006. Pseudomonas savastanoi pv. savastanoi, Causal Agent of Olive Knot Disease in Morocco: Molecular Identification and Biological Control}

Abdelaaziz Bouaichi ${ }^{1.2 *}$, Rachid Benkirane ${ }^{2}$, Khaoula Habbadi $^{2}$, Said El kinany ${ }^{3}$, Hanane Lougraimzi ${ }^{4}$, Mohamed Ou-zine ${ }^{3}$, Soumia Sadik ${ }^{5}$, Abdellatif Benbouazza ${ }^{1}$, and El Hassan Achbani ${ }^{1}$

1 Laboratory of Phytobacteriology and Biocontrol, National Institute of Agronomic Research, Meknes, Morocco; ${ }^{2}$ Laboratory of Botany, Biotechnology and Plant Protection, Faculty of Science, Ibn Tofial University, Kenitra, Morocco; ${ }^{3}$ Laboratory of microbial biotechnology, Faculty of Sciences and Techniques, Sidi Mohamed Ben Abdellah University, Fez, Morocco; ${ }^{4}$ Laboratory of Health, Nutrition and Environment, Faculty of Sciences, Ibn Tofail University, Kenitra, Morocco; ${ }^{5}$ Laboratory of Plant Biotechnology and Molecular Biology, Faculty of Sciences, Moulay Ismail University, Meknes, Morocco. "Corresponding and Presenting Author: bouaichi.inra@gmail.com. 


\section{Abstract}

Olive knot disease caused by Pseudomonas savastanoi pv. savastanoi (PSS) is considered one of the most serious diseases affecting olive trees (Olea europaea $\mathrm{L}$.) in most olive growing regions worldwide and mainly in Mediterranean countries, which can lead to severe damage in olive groves, causing serious losses in terms of production. This study aimed to isolate and identify the organisms causing olive knots on stems and branches in different regions in Morocco as well as biological control with bio-agents like essential oil and antagonistic bacteria. Bacteria were isolated from young knots on selective media. Biochemical tests, pathogenicity tests and PCR were performed to identify the strains causing disease. Fifty-nine isolates were identified as PSS using biochemical, pathogenicity tests and PCR technique using specific primer. Based on the inoculation of olive plants (cv. Arbequina) with isolated strains, twenty-five caused typical knots at the site of inoculation. To control PSS, essential oils (EO) Thymus ciliatus and the Origanum compactum exhibited in vitro inhibition of PSS strains. Their inhibition rates were respectively $56.25 \%$ and $50 \%$. Also, some biocontrol agents, Rahnella aquatilis, Enterobacter ludwiqii, Paenibacillus brasiliensis, and Bacillus subtilis selected from collection of Regional Centre of Agronomic Research revealed in vitro antagonism toward pathogen strains. The most efficient strain, B. subtilis 2515-1 was selected for pots experiment using olive trees (cv. Arbequina). This bacterium significantly reduced number and weight of knots on trees inoculated with two pathogens strains (PSS 2064-8 and PSS 2102-4). Keywords: Pseudomonas savastanoi pv. savastanoi, olive knot disease, molecular identification, biological control, essential oils, antagonistic bacteria, Bacillus subtilis.

\section{P007. Improving the Growth of Date Palm in Moroc- co through the Use of Biofertilizers including the Plant Growth Promising Rhizobacteria (PGPR)}

Oufli $M^{1,2^{*}}$, Elkinany $S^{1}$, Oouz-Ine $M^{1}$, Hilali $H^{2}$, and El Hassan Achbani ${ }^{1}$

1 Laboratoire de Microbiologie et Protection des Plantes CRRA, INRA, Meknès, Morocco; ${ }^{2}$ Laboratoire de Microbiologie, Faculte des Sciences et Techniques, University Mohamed I, Settat, Morocco. "Correspond- ing and Presenting Author: m.oufli@uhp.ac.ma.

\section{Abstract}

Excessive application of agrochemicals to improve agricultural production can cause several problems related to their toxicity to humans, soil and groundwater pollution, and biodiversity loss. The current trend is the reduction in the use of pesticides and mineral fertilizers. To achieve this, the use of rhizobacteria has proven to be a promising approach. Rhizobacteria known as Plant Growth Promising Rhizobacteria (PGPR) are now considered an important means for sustainable agriculture because of their ability to improve yield and fight against biotic and / or abiotic stresses. Which will significantly affect the mitigation of the use of chemicals and thereby reduce their harmful effects. Rhizobacteria are endowed with the potential of biological fixation of nitrogen, which reduces the nitrogen fertilizer supply. Thus, they can be considered as a renewable resource. Recently, the use of plant growth promoting rhizobacteria (PGPR) in agriculture has become an alternative to reducing the harmful effects of chemicals on the environment and providing sustainable agriculture. Keywords : PGPR, Date Palm (Phoenix dactylifera L.).

P008. The moisture sorption isotherms and the Thermophysical characterization of Durum Wheat "Triticum durum L."

Haytem Moussaoui ${ }^{*}$, Idlimam Ali, and Abdelkader Lamharrar

Laboratory of Solar Energy and Medicinal Plants, Teacher's Training College, Cadi Ayyad University, BP 2400 Marrakesh, Morocco. *Corresponding and Presenting Author: haytem.moussaoui@gmail.com

\section{Abstract}

Durum wheat "Triticum durum L." Is a cereal, a wheat variety cultivated since prehistoric times, known for its hard and vitreous grain. It is rich in protein and gluten. Durum wheat is mainly used for pasta, especially in countries of Europe and North America, while in other regions (Middle East and North Africa), it is also used as couscous and different types of bread. The 'Karim' verity is the most popular variety in Morocco and the 
most widespread. It is a cultivar with wide adaptation (different environments: semi-arid and irrigated); it is also early cultivar as well as tolerant to few diseases (septoria and rust). The objective of this work is to develop and validate a trial model of Durum Wheat by identifying the adsorption and desorption curves and optimum water activity. These data constitute an important source of information for establishing stability and the typical storage conditions for the product. When you want to keep a dry product, it is necessary to know the water content of the product needed for its stability. In the hygroscopic field, there is a specific relationship between the moisture content of the product and the relative humidity of the surrounding environment. Therefore, when we want to determine the appropriate water content to keep the product at a certain temperature, it is necessary to know the relative humidity of the surrounding air, which is respected to store properly. Keywords: Durum Wheat, hygroscopic equilibrium, moisture sorption, optimal water activity, sorption isotherms.

P009. Quality Proprieties and the Proceeding Evolution of the Phenols Composition During the Maturity of the Diploid and Triploid Vitis vinifera L. grape in Marrakesh Region

Hicham Jediyi", Khalid Naamani, Abderrehim Ait Elkoch, Nawal Zhar, Naima lemiiber, and A Elkeroumi

Laboratory of Protection and Valorization of Vegetable Resources. Faculty of Sciences Semlalia Marrakech, Cadi Ayyad University, My Abdallah Street, PB: 2390, Marrakesh 40000, Morocco. *Corresponding and Presenting Author: jedhicham@gmail.com.

\section{Abstract}

The objective of this study was to determine the quality proprieties according to the effect of phenolic compounds on grape during maturity of three introduced triploid varieties: Superior, Early sweet, Flame, and two others locale diploid varieties: Abbou and Adari in Oudaya of Marrakesh region. The varieties samples were evaluated by 12 quality parameters and phenols, flavonoids and tannins compounds. The descriptive quality panel found that introduced or locale grape attributes were significant difference $(P \leq 0.05)$ among varieties samples. The statistical test established that the variation of quality proprieties generally and maturity index ( ${ }^{\circ}$ brix/acidity) especially was influenced by the phenolic compounds and the stage of maturity according to fruit maturity. Were the analysis shown as that the phenols, flavonoids and tannins compounds decreased for the triploid and increased up for the diploids during maturity accompanied an increased up of quality proprieties except acidity, and that for of all varieties. The analysis revealed that the phenolic compounds, the rapport of sugar/acidity remain the principals' attributes determinant the maturity of fruits. A classification of fruit maturity stage according to variety and quality proprieties according to phenols composition by PCA analysis is provided. As regards the distribution of variables, we have noted that the juice content, sugars, maturity index and ${ }^{\circ}$ brix for PC2, with phenols, flavonoids and tannins for $\mathrm{PCl}$ are the most variables that have contributed to the discrimination of the grapes according to their stage of maturity and varieties. Keywords: Vine varieties. Quality proprieties. Phenols composition. Maturity grape stages.

\section{P010. Evaluation of Antibacterial Activity of Mentha pulegium Essential Oil Against Strains of Phytopatho- genic Bacteria}

Hanane Lougraimzi ${ }^{{ }^{*}}$, Meryem El Jemli ${ }^{2}$, El Hassan Achbani $^{3}$, abdellah Aghraz ${ }^{4}$, Abdelaaziz Bouaichi ${ }^{5}$, and Mohamed Fadli ${ }^{1}$

1 Laboratory of Health, Nutrition and Environment, Faculty of Sciences, Ibn Tofail University, Kenitra, Morocco; ${ }^{2}$ Laboratory of Pharmacology, Faculty of Medicine and Pharmacy, Mohammed V University, Rabat, Morocco; ${ }^{3}$ Laboratory of Phytobacteriology and Biocontrol, National Institute of Agronomic Research, Meknes, Morocco; ${ }^{4}$ Laboratory of Biotechnology, Protection and Valorisation of Plant Ressources, Faculty of Sciences, Cadi Ayyad University, Semlalia, Morocco; ${ }^{5}$ Laboratory of Botany, Biotechnology and Plant Protection, Faculty of Sciences, Ibn Tofail University, Kenitra, Morocco. "Corresponding and Presenting Author: hananelou04@gmail.com.

\section{Abstract}

Aromatic and medicinal plants have interesting biological activities. The aim of the study is to examine 
the in vitro antimicrobial activitie of essential oil of the leaves of Menhta pulegium (L) against of phytopathogenic bacteria and to propose the alternative solutions based on the use of natural products instead of chemicals while avoiding the presence of toxic substances in the air and soil. The essential oil of this plant was obtained by the hydrodistillation method (Clavenger). The inhibitory effects of this essential oil were tested against phytopathogenic bacteria by using agar disc diffusion and dilution broth methods. The results obtained showed a sensitivity of the different strains to the essential oil of Mentha pulegium, which allowed presenting a therapeutic potential of this plant which could be exploited as a natural antibiotic for the treatment of several infectious diseases caused by these germs and could be helpful in understanding the relationship between traditional remedies and current antibiotics. Keywords: Antibacterial, Essential oil, Mentha pulegium, Sensitivity.

P011. Synergistic Effects of the Inoculation with Plant Growth-Promoting Rhizobacteria and Arbuscular Mycorrhizal Fungus on the Plant Growth in the Field

Anas Raklami ${ }^{1,2^{*}}$, Noura Bechtaoui', Abdel-llah Tahiri ${ }^{1,2}$, Zakaria Es-Salmi ${ }^{2}$, Abdelilah Meddich², and Khalid Oufdou'

' Laboratory of Biology and Biotechnology of Microorganisms, Faculty of Sciences Semlalia, Cadi Ayyad University, PO Box 2390, Marrakech, Morocco; ${ }^{2}$ Laboratory of Biotechnology and Plant Physiology, Faculty of Sciences Semlalia, Cadi Ayyad University, PO Box 2390, Marrakech, Morocco. *Corresponding and Presenting Author: anas.raklami@gmail.com.

\section{Abstract}

Through many researches, arbuscular mycorrhizal fungi (AMF) and plant growths-promoting rhizobacteria (PGPR) have been shown to promote plant growth, yield and nutrient uptake through a range of mechanisms that include improved nutrient acquisition especially phosphorus $(P)$ nutrition, producing plant growth hormones, improving host physiological processes and inhibition of plant pathogens. The purpose of this study was to evaluate the effect of the inoculation with plant growth-promoting rhizobacteria and arbuscular mycorrhizal fungus and their potential to stimulate two of the most widely grown crops over the world. They are not only an important source of proteins in food and feed, but also a significant component of different agrosystems. These plants are the legume plant Vicia faba L. (Aguadulce variety) and the cereal plant Triticum aestivum (Karim variety). The effect of double inoculation, was studied in field experiment in agricultural soil with six treatments, the control without inoculation, PGPR alone, rhizobia alone, mixture of PGPR and rhizobia, AMF alone and a mixture of PGPR, rhizobia and AMF. The greatest effects were observed in the inoculation with a mixture of PGPR-rhizobia-AMF. This inoculation improved the growth parameters (length, fresh and dry weight of shoot and root, number of leaves) of V. faba and T. aestivum. This indicates that the mixed inoculation significantly stimulated the growth of crops and could be used as potential bio-fertilizers to optimize plant growth and yield. Keywords: Arbuscular mycorrhizal fungi, rhizobia, growth, Vicia faba L., Triticum aestivum, fields, PGPR, biofertilizers. Acknowledgments: This work is financially supported by the project PPRCNRST.

P012. Characterization and identification of olive varieties using simple sequence repeats markers

\section{Ahmed El Bakkali* and Abderahman Mekkaoui}

INRA, UR Amélioration des Plantes et Conservation des Ressources Phytogénétiques, Meknès, Morocco. *Corresponding and Presenting Author: ahmed_elbakkali@yahoo.fr.

\section{Abstract}

Olive (Olea europaea L.) is an important fruit species cultivated for oil and canned fruit in the Mediterranean basin. Ex situ conservation is essential for the optimal management and use of genetic resources for both plant breeding and sustainable production. The collection of Ain Taoujdate (AT), Meknes, is one of the ancient ex situ olive germplasm bank in Morocco with 78 varieties from 9 Mediterranean countries. The characterization of all varieties provide useful information for managing olive germplasm to be preserved, exchanged and used in breeding program. In this work, we used 17 preselected microsatellites markers (SSR) to characterize 95 accessions in AT col- 
lection and results were compared to the World Olive Germplasm Bank of Marrakech (WOGBM) database with 554 olive accessions. Over all the analyzed accessions, low genetic diversity was observed in AT collection compared to the WOGBM with 48 and 402 genotypes, respectively, in which 37 genotypes are common between both collections and 11 genotypes exclusively present in AT collection. Additionally, fifty five errors related to mislabeling plantation and high proportion of redundant genotypes for "Picholine Marocaine" and "Frantoio" varieties were revealed. We were able to identify six genotypes considered as molecular variants and 18 cases of synonyms in AT collection such as "Loretana" and "Dritta di Moscufo" varieties. Even with the low genetic diversity identified in the AT collection compared to the WOGBM, 135 and 261 alleles, respectively, the Principal Coordinates Analysis (PCoA) revealed that genotypes of AT collection span the range of all the 405 genotypes identified in the WOGBM. This study highlighted the powerful of SSR markers used to discriminate between olive varieties and represents a preliminary step towards managing the ex situ olive collection, authenticating varieties and developing further association mapping studies. Keywords : Olea europaea, Ain Taoujdate olive collection, World Olive Germplasm Bank of Marrakech, microsatellites (SSR), genetic diversity management.

P013. Analysis of phenotypic diversity of Moroccan barley accessions maintained ex situ at the National Genebank of INRA, Morocco

Ali Sahri ${ }^{{ }^{*}}$, Hassan Ouabbou', Naima Qariouch', Ali Amamou', and Abderrazek Jilal $^{2}$

1 Institut National de la Recherche Agronomique (INRA), Centre Régional de la Recherche Agronomique de Settat, B.P : 589, Settat, Morocco; ${ }^{2}$ Institut National de la Recherche Agronomique(INRA), Centre Régional de la Recherche Agronomique de Rabat, B.P : 6356 - Rabat, Instituts, Morocco. *Corresponding and Presenting Author: sahriali@gmail.com.

\section{Abstract}

Morocco has long been considered one of the major centers of diversity for barley (Hordeum vulgare L.). It is one of the most important traditional crop and major source for proteins and minerals, in addition to its multiple uses as food and feed. The National Genebank of INRA-Morocco based in Settat conserves and manages more than 3000 barley accessions (representing old cultivars and local landraces) collected from the different regions of Morocco. The characterization of diversity in barley germplasm is very important for utilizing the collection efficiently and effectively. In this study, a representative collection of 200 accessions of barley landraces, belonging to eight different agro ecological regions of Morocco, were analyzed for its diversity of ten agro-morphological traits. Based on Shannon-Weaver diversity indices $\left(H^{\prime}\right)$, Moroccan barley landraces display an indice of 0.66 , indicating the presence of significant degree of variability among the studied populations conserved at the National Genebank. For the agroecological regions, the highest mean diversity index $\left(H^{\prime}=0.70\right)$ was observed for accessions from the Atlas and the Rif mountains. A multiple correspondence analysis (MCA) was carried out on the basis of the ten agro-morphological traits to define the structure of the diversity. The three first axes of the MCA explained $38 \%$ of the total variance in the collection and separated the accessions into two main groups. The first group includes the accessions from the Rif and Atlas Mountains and the second group includes the accessions from the other agro ecological regions. The results of the MCA indicated that the geographical proximity and altitude play a main function in the discrimination and the structure of the studied barley accessions. The information generated from this study (high variability) could be used in conventional breeding programs and in situ conservation. Keywords: Barley accessions, national genebank, agro-morphological traits, Shannon-weaver diversity index, variability, multiple correspondence analysis.

\section{P014. Effects of Five Biostimulants on Peach Production and Quality in Sais Plain (Morocco)}

Meriame Laita ${ }^{1,2}$, Zerhoune Messaoudi ${ }^{-*}$, Rachid Benkirane $^{2}$, and Lhoussain Ait Haddou'

1 Department of Arboriculture-Viticulture, National School of Agriculture, B.P. S40, Meknes, Morocco; ${ }^{2}$ Botanical, Biotechnology and Plant Protection Laboratory, Faculty of Sciences, University Ibn Toufail, BP 133, Kénitra, Morocco. *Corresponding and Present- 
ing Author: messaoudiz@yahoo.fr.

\section{Abstract}

Improvement of production and quality is among the biggest challenges of peach growing under calcareous soil and dry and hot summer conditions of Sais (Morocco). High soil pH induces low micronutrients uptake which favors leaf chlorosis and extreme temperature and dry air during end of spring and summer time altogether with other conditions induce high incidence of fruit drop and uncertain fruit quality. The present experiment was carried out during 2017 season in a conventional orchard of the Zee lady variety of peach (the rootstock is the almond) in Sais, Morocco with an aim to evaluate biostimulant effects of five products on peach yield and quality. Six variants namely Vitazyme, Protifert LMW 10, Protifert LMW 6,3, Protifert Zinc and Naturfer biostimulants and Control (unsprayed trees) were compared according to a completely randomized design with 3 repetitions. Data collected indicated a remarkable effect of biostimulant on fruit number per tree and harvest size and some pomological fruit parameters namely fruit weight, dimensions, core weight and core/flesh ratio. Keywords: Peach, fruit drop, fruit quality, biostimulant, pomological.

P015. New insights of climate change effects on codling moth (Cydia pomonella L.) voltinism in apple orchards and perspectives of efficient control strategy

\section{Salma El Iraqui $i^{*}$ and $M^{\prime}$ Hamed Hmimina ${ }^{2 \S}$}

1 Plant Protection Department, National Institute of Agricultural Research, Meknès Regional Center, BP 578, Meknès, Morocco; ${ }^{2}$ Zoology Department, Institut Agronomique et Véterinaire Hassan II, B.P 6202, Madinat Al Irfane, Rabat-Institutes, Rabat, Morocco. *Presenting Author: iraquisalma@gmail.com. ${ }^{\S}$ Corresponding author: hmimina@yahoo.fr.

\section{Abstract}

Codling moth (Cydia pomonella L.) is the worldwide k\&ey pest of apples causing heavy economic losses in fruit production. In Morocco, during the last decades, a massive flight of moths leads to more than 12 chemical treatments per year. To improve the con- trol of Cydia pomonella, this study aims to accurate its voltinism in relation with temperature variations. The progress of codling moth generations was investigated during 11 years in a Moroccan apple orchard based on catches of sexual traps. The degree days (DD) method was also investigated to verify its adaptability to phenological forecasting models. The impact of temperatures of two periods was investigated: temperatures matching with the beginning of the life cycle of Cydia pomonella (April and May) and temperatures prevailing in the period conducive to diapause (July 10 to early August 5). Results revealed that the voltinism of codling moth had evolved in 2, 3 to 4 generations, depending on the season's temperature. Three complete generations and a fourth partial one characterized mostly its life cycle. The accomplishment of the fourth generation is highly expected in seasons recording high temperatures in April and May (360DD: case of 2014 and 2015). These high temperatures allow an earlier and faster development of the first generation which impacts the progress of the following ones, extends the presence of the insect and consequently increases its voltinism. Moreover, temperatures during, July to early August, are always high in Morocco and thus inhibit insect diapause and extends its duration of presence until the harvest. Finally, the estimated generations, based on DD method, were in concordance with codling moth flight dynamics, supporting the use of phenological models. Climate scenarios predict a rise in temperatures in the future, favoring the accomplishment of the fourth generation and consequently more damages in productions and more insecticides used. Keywords: Cydia pomonella L., voltinism, temperatures, degree days.

\section{P016. Isolation and Identification of Aerobic Bacterial Community in Earthworm (Lumbricus terrestris) chlor- agogen tissue}

Lamia Yakkou**, Maryam Chelkha, Mohammed Raouane, Souad Amghar, and Abdellatif El Harti

Reserch Team : Lumbricidae, Improving Soil Productivity and Environment (LAPSE), Centre "Eau, Ressources Naturelles, Environnement et Développement Durable (CERN2D)" Mohammed V University in Rabat, Ecole Normale Supérieure (ENS). Avenue Med Belhassan El Ouazani. BP 5118 , Takaddoum-Rabat, Morocco. 
"Corresponding and Presenting Author: Yakkou.lam@ gmail.com.

\section{Abstract}

Microorganismes living in association with earthworms have a fundamental ecological and agronomic interest. Several studies have demonstrated the involvement of these invertebrates' intestinal flora in improving soil productivity, particularly in the processes of humification, decomposition and recycling of organic matter. In this work, we investigated the isolation and identification of other microbial species living in association with earthworms (Lumbricus terrestris) in organs other than the gastrointestinal tract. The microscopic observation of chloragogen tissue smears, taken by dissection of the worm, revealed the presence of microbial entities of various shapes and sizes. Isolation and cultivation made it possible to inventory seven bacterial strains. Phenotypic and genotypic studies (PCR amplification of $16 \mathrm{~S}$ rDNA), allowed identification: Pseudomonas fluorescens, Pseudomonas montelii, Citrobacter freundii, Enterobacter aerogenes, Staphylococcus epidermis, Teribacillus aidengensis and Pseudomonas rhizosphaerae. Among the identified bacterial strains, the three species of Pseudomonas (fluorescens, montelii and rhizosphaerae), along with Enterobacter aerogenes are all of ecological and agronomic interest, particularly in improving plant productivity and soil bioremediation. Keywords: Lumbricus terrestris, chloragogen tissue, Microorganisms, PCR-16S rDNA, Soil productivity.

P017. Study of chemical composition and antibacterial activity of Thymbra capitata essential oil and its use in carrot juice preservation

Saoulajan Charfi ${ }^{*}$, Nadia Boujida, Jamal Abrini, and Nadia Skali Senhaji

Laboratory of Biotechnology and Applied Microbiology, Team Biotechnology and applied Microbiology, Department of Biology, Faculty of Science, Abdelmalek Essaadi University, Tetouan, Morocco. *Corresponding and Presenting Author: sawlajan@gmail. com.

\section{Abstract}

The aim of this work was to investigate the composition and antibacterial activity of Moroccan Thymbra capitata essential oil (TCEO) and to evaluate its application as food preservative in carrot juice conserved at 20 and $5^{\circ} \mathrm{C}$. Chemical composition was analyzed by GC/MS. TCEO antibacterial activity was evaluated by diffusion method and microtitration assays against Escherichia coli O157:H7 CECT 4267, Listeria monocytogenes CECT 4032 and Staphylococcus aureus CECT 976. In carrot juice, TCEO activity was evaluated against these pathogens during 30 days storage at 20 and $5^{\circ} \mathrm{C}$. Analysis of TCEO chemical composition showed that the main constituent of TCEO was carvacrol $(75.52 \%)$, followed by p-cymene $(9.75 \%)$ and $\beta$-caryophyllene (6.45\%). TCEO exhibited an antibacterial activity in vitro against the tested bacteria. In carrot juice, TCEO was able to inhibit the growth of all pathogenic bacteria at both storage conditions. L. monocytogenes was the most sensitive strain to TCEO when tested with diffusion method and in carrot juice, followed by S. aureus and E. coli O157:H7. TCEO was more efficient against $\mathrm{E}$. coli $\mathrm{O} 157: \mathrm{H} 7$ in carrot juice conserved at $5^{\circ} \mathrm{C}$ than at $20^{\circ} \mathrm{C}$. On the other hand, no significant difference between storage temperatures was noted for L. monocytogenes and S. aureus. These results show the ability of TCEO to inhibit pathogenic bacteria and to maintain the safety of carrot juice. Keywords: Thymbra capitata essential oil, chemical composition, antibacterial activity, carrot juice.

P018. Effect of six aromatic plants essential oils on storage keeping quality of fresh strawberries and raspberries

Asmaa Mziouid ${ }^{2}$, Bouchra Chebli1, and El Hassan Mayad $^{2}$

1 Laboratory of Mechanic, Process, Energy and Environmental, Biotechnology and Environmental Engineering Team, National School of Applied Sciences, Ibn Zohr University, PO Box: $1136 /$ S, Agadir, Moroc$\mathrm{CO}^{2}{ }^{2}$ Laboratory of Biotechnologies and Valorization of Natural Resources, Faculty of Sciences - Agadir, Ibn Zohr University, Agadir, Morocco. "Corresponding and Presenting Author: mmesadek@hotmail.fr. 


\section{Abstract}

Six essential oils (EOs) were hydrodistillated in a Clevenger-type apparatus from: Artemisia absinthium, Artemisia herba-alba, Cladanthus arabicus, Lavendula dentata, Thymus broussonnetii and Thymus leptobotrys plants growing in Morocco. The EOs were tested for their antifungal activity first in vitro against Botrytis cinerea using agar dilution and disk diffusion methods. And then the EOs showing the lowest minimum inhibitory concentration were tested for their ability to extend the shelf life of fresh strawberries and raspberries, conserved at $4^{\circ} \mathrm{C}$. In vitro, Cladanthus arabicus had the lower antifungal property. The essential oils of Thymus leptobotrys and Thymus broussonnetii inhibited the growth of fungus at 250 $\mu \mathrm{l} / \mathrm{L}$ and $500 \mu \mathrm{l} / \mathrm{L}$ respectively and were found to be more efficient preservatives for strawberries and raspberries during 11 days of cold storage. The results suggest that essential oils vapour may improve fruit quality during fresh strawberries and raspberries storage and transit. Keywords: Artemisia absinthium; Artemisia herba-alba;Cladanthus arabicus; Lavendula dentate; Thymus broussonnetii ; Thymus leptobotrys; Botrytis cinerea; Essential oils; shelf life; strawberries; raspberries

\section{P019. Fertilization practiced by olive growers of Sais and its impact on olive orchards.}

Karima Bouhafa ${ }^{1 *}$, Lhoussaine Moughli², Khadija Bouchoufi', and Abdelkader Sdouq'

1 Regional Agricultural Research Center, P.O.Box 578, Meknes, Morocco; ${ }^{2}$ Department of Soil Science, Hassan II Institute of Agronomy and Veterinary Medicine, P.O.Box 6202, Rabats-Instituts, Rabat, Morocco. 'Corresponding and Presenting Author: bouhafakarima@ gmail.com.

\section{Abstract}

A study on fertilization practices adopted by farmers was carried out, through surveys, soil and leaf analysis, at 58 olive orchards randomly selected in Sais region. Soil samples were collected from the 0-30 and $30-60 \mathrm{~cm}$ layers during December-January. Leaf samples were also collected during the same period. The results of the surveys showed that about $48 \%$ of the olive growers do not use any mineral fertilizer for their olive trees. This has affected the olive yield, which averaged $3 \mathrm{~T} / \mathrm{ha}$ in fertilized orchards and 1.9 T / ha in unfertilized orchards. Nitrogen is the most provided by the farmers with an average dose of $37 \mathrm{~kg} \mathrm{~N} / \mathrm{ha}$, followed by phosphorus with $20 \mathrm{~kg}$ $\mathrm{P} 2 \mathrm{O} 5$ / ha and potassium with $9 \mathrm{~kg} \mathrm{~K} 2 \mathrm{O} / \mathrm{ha}$. These doses are low for the olive tree. In addition, phosphorus is provided by some farmers at very high doses that have reached $138 \mathrm{~kg}$ P2O5 / ha. Times of fertilizer intakes were generally concentrated over the period from January to April, indicating a lack of inputs during other periods where the need for mineral elements being important for the olive tree such as the fruit enlargement phase. These data indicate the existence of a failure in the fertilization practices adopted by olive growers concerning both the fertilizer doses brought and the timing of fertilizer inputs. Soil tests showed that $50 \%$ and $84.5 \%$ of the soils studied are low in phosphorus, respectively, for the $0-30 \mathrm{~cm}$ and $30-60 \mathrm{~cm}$ layers. Soil potassium levels are low to medium in $15.5 \%$ and $55 \%$ of olive orchards, respectively, for $0-30 \mathrm{~cm}$ and $30-60 \mathrm{~cm}$ soil layers. These soil analysis results from these olive groves confirmed the existence of a failure in the fertilization practices adopted by farmers. Leaf analyzes showed that all the studied orchards require nitrogen inputs and nearly $91 \%$ of orchards require potassic fertilization. For phosphorus, only a third of the orchards sampled need an application. This study showed that fertilizer is not well controlled by olive growers and olive orchards are not well fed with essential elements for their production. Keywords: Fertilization, survey, Sais, soil fertility, nitrogen, phosphorus, potassium.

\section{P020. Impact of PGPR solubilizing phosphate bacteria on growth and phosphorus uptake of legumineuse plan- te Vicia faba $L$.}

Bechtaoui Noura ${ }^{1 *}$, Raklami Anas', El Alaoui Abdelkhalek', Benidire Loubna', Göttfert Michael ${ }^{2}$, and Oufdou Khalid'

${ }^{1}$ Laboratory of Biology and Biotechnology of Microorganisms, Faculty of Sciences Semlalia, Cadi Ayyad University, PO Box 2390, Marrakech, Morocco;

${ }^{2}$ Technische Universität Dresden, Institut für Genetik, Helmholtzstr. 10, D-01069 Dresden, Germay. "Corresponding and Presenting Author: noura.bechtaoui@ 
gmail.com.

\section{Abstract}

Phosphorus $(P)$ is a critical element for plant growth; its deficit severely limits crop yields. A considerable amount of phosphate is concentrated in the upper part of soil in mineral or organic composition. However, these forms are no longer available, because they rapidly react with soil components, therefore only a small quantity of soluble phosphate can actually be absorbed by the roots. A low amount of phosphate limits plant growth, especially in periods of high use, flowering and fruiting stage. Some of the rhizospheric bacteria have the ability to solubilize the complex forms of $P$ in the soil and make it available to plants. These bacteria, in addition to improve phosphate nutrition, my also promote plant growth by producing phytohormones and some other beneficial substances such as siderophores and antibiotic compounds. The aim of the present study is to screen the phosphate solubilizing bacteria from agricultural soils and to test the synergistic or antagonistic effects of a mixed inoculation on the growth of the legume Vicia faba L. The in vitro test of phosphate solubilizing capacity of strains used during this study was carried out on NBRIY medium (Nautiyal, 1999). The capacity of bacteria to produce siderophores, auxins and exopolysaccharides was also analysed. All of the tested strains produce a significant amount of siderophores and auxins. Inoculation experiments with faba bean revealed that some symbiotic combinations result in an increase in biomass and $\mathrm{P}$ content of the inoculated plants. During this study, we prove through our laboratory tests and under greenhouse experimentation, that phosphate solubilizing bacteria could be used as potential bio-fertilizers to optimize the phosphorus supply of plants in deficient soils. Keywords: solubilizing PGPR bacteria, rhizosphere, siderophores, Vicia faba.

P021. Selection and characterization of rhizobacteria isolated from the rhizosphere of peanut (Arachis hypogaea $L$.

Ouissale Chaghouaoui ${ }^{*}$, Imane Achkouk, Amin Laglaoui, Mohammed Bakkali, Abdelhay Arakrak

Equipe de Recherche de Biotechnologies et Gé- nie des Biomolécules (ERBGB), Faculté des Sciences et Techniques de Tanger B.P. 416, Tanger, Morocco. ${ }^{*}$ Corresponding and Presenting Author: chaghouaouiovissale@gmail.com.

\section{Abstract}

The rhizosphere supports the development and the activity of a microbial community including the plant growth-promoting bacteria or the PGPR, they play an important role in the biofertilisation of where the interest of the use as alternatives of chemical fertilizers in order to respond to the need of food for the world population, they may intervene either directly by providing the nutrients or by producing growth regulators or indirectly by removing the phytopathogenic agents. In this context of sustainable agriculture and the protection of the environment, this work led to the isolation and characterization of the solubilising Bacteria The phosphate (BSP) associated with three varieties of peanuts (Arachis hypogaea) (Jumbo, Valencia and Beldi) grown in pots under greenhouse conditions for 28 days. Various tests have been carried out on the isolates studied to evaluate the different phytobenificial properties in vitro. A total of 79 isolates on 300 isolates tested (26.33\%) have been capable of dissolving the tricalcium phosphate (TPC) on mid Pikovskaya solid, and whose diameters of halo have been between 1 and $10 \mathrm{~mm}$. The isolates with diameters of solubilization $\geq 4 \mathrm{~mm}$ (49 isolates) were selected and evaluated for the production of indole acetic acid, siderophores, and ammonia which the percentages are respectively $69.38 \%, 91.83 \%$ and 100\%. 6 BSP (JP19, JR33, JR41, JR48, BP49 and BR44) have been tested for the solubilization of the PTC in mid PVK liquid with the highest concentration (49.96 $0.85 \mathrm{mg} / \mathrm{L}$ ) has been registered by JR19. Keywords: Arachis hypogaea, biofertilisation, $B S P$, PGPR, rhizosphere.

\section{P022. Pesticide wastes management in some horticul-} tural farms in the Souss Massa

\section{Abderrahim Hormatallah'}

Laboratoire des pesticides. Institut Agronomique et Vétérinaire Hassan II, Complexe Horticole d'Agadir, BP 18/S. Agadir, Morocco. "Corresponding and Presenting Author: phytopharmacie@yahoo.fr. 


\section{Abstract}

Horticulture is one of the most relevant segments of the Moroccan agriculture sector. Morocco country reaches the self-sufficiency in vegetable production. This sector provides also an employment for the working rural population and important source of hand currency towards exportation to the European Union, Canada, Russia and USA. The intensive conditions of the horticulture crops (citrus, tomato, pepper, green bean...) seem to be favorable to many insects (leaf miners, thrips, Whiteflies ..) and the development of some diseases (powdery mildew, downy mildew, gray mold...) that cause more damage and affecting the yield quality. Growers are often applying pesticides to protect their production and the yield quality. Pesticide waste disposal is a concern for Moroccan horticultural producers. In order to meet the objectives of "Green Morocco Plan", to complain to national Legislation (Law 42-95 regarding pesticides regulations, Law 28-00 related to waste management and their disposal) and international regulations (FAO's Code of Conduct for Pesticide use and disposal) and the requirements of Quality Control standards of agricultural products and the environmental management (GlobalGap, Nature'choice, ISO 14000, BRC, it will be very important to know the practices used by farmers to manage pesticide wastes. 60 horticultural producers of the Souss Massa region were interviewed personally with a questionnaire focused on Pesticides waste Management. The Souss Massa, located in the south Atlantic coastal, is a major horticultural producing and exporting area of Morocco. The survey indicated that pesticide wastes management depends mainly on the certification of the farms. The majority of pesticide wastes were related to empty pesticide containers and pesticide effluents. All farmers are aware of human health risk and the environment pollution potential of pesticide wastes. The producers are face problems in terms of eliminating the empty containers due to the absence of laws and facilities for pesticides containers recycling. Certified farms make triple rinsed pesticide containers and stored them under lock and key, or they reuse them for cultural operations after cleaning. In the case of the non-certified farms, the empty containers are burned or are sold via informal ways. Burning plastic bottles and pesticide box can lead to the formation of toxic gas in the air. The component 2 of GCP /MOR/041/
GFF project in Morocco will be developed a management system including outreach campaign to users for triple-rinsing and safe storage, collection and recycling of all types of containers, firstly through a regional pilot program in the Souss Massa. Concerning leftover spray solutions, most farmers (73\%) was reported that they apply them to the border lines of the field area or to weed grasses until the spraying tank is empty. $16 \%$ of farmers release the leftover spray solutions into an evaporation area and $11 \%$ were used a bioremediation system in phytobac or heliosec. Keywords: Pesticide wastes, empty pesticide containers, pesticide effluents, phytobac, heliosec, Souss-Massa, GCP /MOR/041/GFF project, FAO.

\section{P023. Effect of mineral fertilization on olive quality}

Karima Bouhafa ${ }^{1 *}$, Lhoussaine Moughli ${ }^{2}$, Khadija Bouchoufil , Ahmed Douaik ${ }^{3}$, and Khalid Daoui'

${ }^{1}$ Regional Agricultural Research Center, P.O. Box 578, Meknes, Morocco; ${ }^{2}$ Department of Soil Science, Hassan II Institute of Agronomy and Veterinary Medicine, P.O. Box 6202, Rabats-Institutes, Rabat, Morocco; ${ }^{3}$ Regional Agricultural Research Center, PO Box 6356, Avenue Mohamed Belarbi Alaoui, Rabat, Morocco. *Corresponding and Presenting Author: bouhafakarima@gmail.com.

\section{Abstract}

Two fertilization trials were conducted in two olive orchards under rainfed conditions in Oued Amlil (S1) and Ain Taoujdat (S2). The objective was to study the effect of nitrogen $(N)$, phosphate $(P)$ and potassium (K) fertilizer inputs on the olive quality. The experimental design adopted for these trials is a factorial in incomplete random blocks. Four doses of each of the elements $N, P$ and $K$ were tested: $0,0.25,0.5$ and 1 $\mathrm{kg} \mathrm{N} /$ tree / year; $0,0.12,0.25$ and $0.5 \mathrm{~kg}$ P2O5 / tree / year and 0, 0.5, 1 and $2 \mathrm{~kg} \mathrm{K2O} \mathrm{/} \mathrm{tree} \mathrm{/}$ year. The average levels of olive oil, expressed as $\%$ dry matter, were 28.3 and $37.3 \%$, respectively, in S1 and S2. The effect of nitrogen on olive oil content was insignificant at $\mathrm{S} 1$ and highly significant at S2. At the S2, the nitrogen supply induced a decrease in the oil content and the control recorded the maximum level of olive oil (38.5\%). Phosphorus had no significant effect on olive oil content in S1. On the other hand, 
the input of $P$ caused a significant increase in the oil content in S2. Potassium did not affect the olive oil content at both sites. Regarding the effects of mineral elements on the olive oil quality, it is noted that nitrogen is the only element that significantly affected the two measured parameters (peroxide index and acidity). The intake of $\mathrm{N}$ induced an increase of the peroxide index to reach the maximum value by the dose $0.5 \mathrm{~kg} \mathrm{~N} /$ tree / year in the site 1, and by the dose $1 \mathrm{~kg} \mathrm{~N} /$ tree / year in the site 2. On the other hand, a decrease in the acidity level of the olive oil was observed with the addition of nitrogen at S2 where the best quality of the olive oil (minimum acidity) was obtained by the dose $0.5 \mathrm{~kg} \mathrm{~N} / \mathrm{ha} / \mathrm{yr}$. The effect of nitrogen on olive oil acidity was not significant at S1. Keywords: Olive, nitrogen, phosphorus, potassium, acidity, peroxide index.

\section{P024. Assessment of beta-glucan and micronutrients in a collection of barley's wild relatives from ICADRA's gene bank}

Fadwa Elouadi ${ }^{*}$, A. Amri ${ }^{2}$, A. Jilal ${ }^{3}$, G. Salih ${ }^{3}$, and M. Ibriz'

' Laboratoire de Génétique et biométrie, Faculté des Sciences. Université Ibn Tofail, B.P. 133, Kénitra. Morocco; ${ }^{2}$ International Center for Agricultural Research in the Dry Areas, Rue Hafiane Cherkaoui, Rabat, Morocco. PO Box 6299, Rabat Institutes, Rabat, Morocco; ${ }^{3}$ RU Plant Breeding, Conservation and Valorization of Plant Genetic Resources, INRA, RCAR-Rabat, P.O. Box 6570, Rabat Institutes, Rabat, Morocco. "Corresponding and Presenting Author: elouadi.fadwa@ gmail.com.

\section{Abstract}

Barley is one of the oldest domesticated crop in the word, although barley food uses remains important only in some cultures particularly in Asia and northern Africa. Whereas recently the consumer has become aware of the interest of barley as a food grain because of his impressive health benefits, and as a major source of soluble and insoluble fibers, also his antioxidant activity. The objective of this study is to investigate the genetic variation in a population of barley's wild relatives. Beta-glucan, and micronutrient concentration were determined, as well the identification of quantitative trait loci (QTL) associated with these traits in a population of wild barley from ICARD's gene bank using a genome-wide association study (GWAS). Accessions of wild relatives were grown under greenhouse for genotyping, and field conditions for multiplication (2015-2016/2016-2017), characterized for beta-glucan, and micronutrient concentration, and then genotyped with $50 \mathrm{~K}$ single nucleotide polymorphism (SNP) markers. The population exhibited a wide range of variation for these traits, further some accessions had shown high levels of beta-glucan and micronutrient. This study demonstrates the variation of beta-glucan and micronutrient concentration in wild barley and the interest of genotyping to underline genes controlling these traits. Key words: barley, beta-glucan, micronutrient, genome-wide association, quantitative trait loci.

\section{P025. Time Evolution of Transpiration and Heat Transfer Inside Arch Greenhouse Crop Using Computational Fluid Dynamics}

Allal Senhaii ${ }^{{ }^{*}}$, Mhamed Mouqallid', and Hassan Majdoubi ${ }^{1,2}$

${ }^{1}$ Equipe de recherche en Energétique et Mécanique des Fluides, ENSAM, Meknès, Morocco; ${ }^{2}$ CRMEF FesMeknès, Meknès, Morocco. "Corresponding and Presenting Author: senhaii145@gmail.com.

\section{Abstract}

The most important factor affecting plant growth and development in greenhouses is solar radiation because, in addition to its indirect effect on the distribution of the greenhouse's internal climate, it governs two important physiological mechanisms of plants, namely the transpiration and photosynthesis. The amount of solar radiation entering the greenhouse depends on the design of the greenhouse, the thermophysical and optical properties of the material, and the weather conditions, there are important variations in the amount of solar radiation reaching the crop, which strongly impacts climate and therefore crop activity. The aim of this paper is to develop a specific numerical submodel to simulate the distributed transpiration and microclimate inside greenhouses under day-time conditions. The numerical simulation was adopted in three dimensions (CFD), using the computer 
code Fluent v6.3.26 which is based on solving the Navier-Stokes equations. These equations are solved in the presence of turbulence model $(k-\varepsilon)$ and a biband radiative submodel distinguishing short and long wavelength radiations was employed through a Discrete Ordinates (DO) model to solve the equation of heat transfer by radiation (ETR). Simulations were carried out for a greenhouse of $810 \mathrm{~m} 2$ equipped with continuous roof vents. The analysis focuses on the heterogeneity of the distributions of climatic parameters (speed, temperature and humidity). But the main point concerns the heterogeneity of the radiation reaching the canopy and the latent and sensitive heat flux inside the culture. Keywords: Solar radiation, transpiration, CFD, crop, greenhouse, microclimate.

P026. Micropropagation and Plantlet Regeneration of Saffrom (Crocus sativus L.)

Meryem Maiski ${ }^{*}$, O. Lamsaouri, M. Fakiri, and Y. El Goumi

Laboratoire de recherche Agroalimentaire et Santé, Faculté des Sciences et Techniques, Université Hassan 1er, Settat, Morocco. "Corresponding and Presenting Author: meryem.maiskii@gmail.com.

\section{Abstract}

This study consists in evaluating various methods of $\mathrm{mi}-$ cropropagation of saffron (Crocus sativus L.).different protocols of surface sterilization have been tested with the aim to decrease the contamination porcentage. The effect of growth regulators combinations has been also tested on the induction of caulogenesis. The stirilization procedures tested did not show their efficiency on corms. However, in the last protocol, the explants showed a significant lower contamination rate $(2,44 \%)$. The growth regulators used in the induction phase had allowed obtaining the best results of caulogenesis (96\%). Moreaver, it was observed that the use of an ather combination of cytokinin and auxin was favorable for buds' growth. The yield was 1,06 buds per explant newly formed.
P028. Variability Analysis of the Seeds and Oil Yields of Several Accessions of Citrullus colocynthis collected in Morocco

\section{Abdelhamid Ben Moumou ${ }^{*}$ and Saïd El Madidi ${ }^{\S}$}

Biometrics and Bio Resources, Laboratory Biotechnologies and Natural Resources Development (LBVRN), Faculty of Sciences, University Ibn Zohr, Agadir, Morocco. "Presenting author: benmoumou36@yahoo.fr. §Corresponding author: s.elmadidi@uiz.ac.ma.

\section{Abstract}

Citrullus colocynthis has been garnering interest in recent times as a potential biodiesel feedstock crop due to its high seed oil content. This plant is a drought tolerant species, as established from the fact that it grows as a weed in arid and semi-arid lands. Widely distributed in the Sahara-Arabian desert in Africa and the Mediterranean region. It is one of the few spontaneous species of plants that grow in arid conditions in southern Mediterranean countries and Middle Eastern countries and could be used as raw material for biodiesel. In addition to its potential as a biodiesel feedstock the root and callus extracts of $C$. colocynthis have been reported to have anti-microbial, anti-inflammatory,anti-diabetic and anti-oxidant effects. C. Colocynthis is a rich source of functionally important bioactive compounds and therapeutics such as polyphenols, glycosides, triterpenes and cucurbitacins and its fruit has been widely used for the treatment of many diseases including diabetes, rheumatism, paronychia, ulcer and cancer. The ripe fruits of $C$. colocynthis were harvested in 12 different localities in Morocco. Geographic coordinates, morphological characteristics were recorded. The measured traits are: fruit weight (FW), seed weight (SW), seed number (SN), weight of 100 seeds, and oil yield. The results of anova (GLM) show significant and very highly significant effects according to the characters measured. Significant variability was observed for seed and oil yields. This variability could be exploited for the selection of accessions with high oil yields. Keywords: Citrullus colocynthis, variability, seeds, oil yield, Morocco. 
P029. Climate change and land suitability in central Morocco: impact and adaptation

\section{A Labaioui ${ }^{i^{*}}, K$ Bouchoufi ${ }^{2}$, and C Kassimi ${ }^{2}$}

1 Regional Center of Agricultural Research, Meknes, Morocco; ${ }^{2}$ Soil Analysis Laboratory, Regional Center of Agricultural Research, Meknes, Morocco. "Corresponding and Presenting Author: amlabaioui@gmail. com.

\section{Abstract}

The climate change will bring about changes in the areas suited to the growing of several species. The effects in terms of production potentiality will be very variable according to the region and the vegetal covers. They can come out by a moderate stimulation in moderate climate and for a warming limited to $2^{\circ}-3^{\circ} \mathrm{C}$. Beyond, as for the low latitudes, excessive temperatures and an increased frequency of the droughts will lead to negative effects. In our study, we used current and future climatic data up to the year 2050, the Ecocrop Model integrated in the Diva-Gis software and soil map to predict the impact of the climate change on the land suitability of the Meknes area in central Morocco, for wheat, chickpea, the olive and the almond trees, so that these changes can be taken into account in the planning of the strategies of adaptation to the climate change. The results show that for wheat, the olive and the almond trees, the aptitude of the soil will increase in spite of the Increase of the temperature and the reduction in rainfall. Consequently, the strategies of development and of improvement of these three crops will not resist the important constraints related to the climatic conditions and should concentrate on the improvement of agronomic management and on the reinforcement of the capacities of farmers by including the techniques of control of the pests and diseases. For chickpea, however, land suitability will decrease, with very marginal land increasing from $5 \%$ to $75 \%$ of the total land area. So, the areas that will lose planting capacity need to be maintained, using targeted strategies such as, irrigation and switching to more drought-resistant varieties and or changing planting date. While areas that are likely to suffer from a significant decrease in aptitude should begin to diversify, moving to other crops as soon as chickpea is no longer appropriate.
Keywords: climate change, land suitability, Morocco, adaptation.

P030. Evaluation of Five Mutants of Rapeseed (Brassica napus L.) for Earliness, Agronomic Traits and Response to Changing Environments in M2 Population

Souhail Channaoui ${ }^{1,2}$, Mostapha Labhilili ${ }^{3}$, Mohamed Mouhib ${ }^{4}$, Hamid Mazouz ${ }^{2}$, Mohamed El Fechtali', and Abdelghani Nabloussi ${ }^{{ }^{*}}$

1 Research Unit of Plant Breeding and Plant Genetic Resources Conservation, National Institute of Agricultural Research, Regional Agricultural Research Center of Meknes, Morocco; 2 Laboratory of Plant Biotechnology and Molecular Biology, Department of Biology, Faculty of Science, University Moulay Ismail, Morocco; 3 Research Unit of Plant Biotechnology, National Institute of Agricultural Research, Regional Agricultural Research Center of Rabat, Morocco; 4 Research Unit of Nuclear Techniques, Environment and Quality, Regional Agricultural Research Center of Tangier, Morocco. "Corresponding and Presenting Author: abdelghani.nabloussi@gmail.com.

\section{Abstract}

Mutagenesis breeding is an efficient strategy to improve rapeseed for several productivity and quality parameters. Five new $M 2$ mutant populations $(\mathrm{H} 2 \mathrm{M}$ $1, \mathrm{H} 2 \mathrm{M}-2, \mathrm{H} 2 \mathrm{M}-3, \mathrm{H} 2 \mathrm{M}-4$ and $\mathrm{H} 2 \mathrm{M}-5$ ) were selected from gamma irradiated and Ethyl Methane Sulphonate (EMS) treated material. A field experiment was conducted in two INRA Experimental Stations, at Douyet and Sidi Allal Tazi, to evaluate rapeseed mutants for earliness, agronomic traits and response to changing environments. The variety 'INRA-CZH2' (wild type) was used as a check. Data on days to flowering, days to maturity, plant height, number of branches/ plant, number of pods/plant, number of seeds/pod, pod length, pod diameter and 1000-seed weight were recorded. All the parameters showed significant differences among the mutants studied for both environments. Mutant $\mathrm{H} 2 \mathrm{M}-1$ was earlier to flowering and maturity and was characterized by a higher number of pods/plant than the check. Mutants H2M-2, H2M4 and $\mathrm{H} 2 \mathrm{M}-5$ produced higher number of branches per plant and 1000-seed weight, compared to the check variety, whilst Mutant H2M-3 exhibited a re- 
duced plant height and stature. Among the five mutant lines, H2M-1 seemed to be the most interesting and further investigations and trials are needed in order to confirm its performance and stability as a promising and useful germplasm for releasing a new rapeseed variety. Keywords: Rapeseed, mutants, environments, earliness, seed yield.

P031. Effect of organic fertilizer and commercial arbuscular mycorrhizal fungi on the growth of micropropagated date palm cv. Feggouss

El Kinany Said ${ }^{1,2,5^{*}}$, Achbani El hassan2, Faggroud Mohamed3, Ouahmane Lahcen4, El Hilali Rania1,4, Haggoud Abdellatif, and Bouamri Rachid ${ }^{1 \S}$

1 Department of Plant and Environment Protection, National School of Agriculture, Meknes, Morocco; 2 Laboratory of Plant Protection URPP-National Institute for Agricultural Research, Meknes, Morocco; 3 Department of Agronomy, National School of Agriculture, Meknes, Morocco; 4 Laboratory of Ecology \& Environment, Faculty of Sciences Semlalia, Cadi Ayyad University, Marrakesh, Morocco; 5 Laboratory of Microbial Biotechnology, Sidi Mohamed Ben Abdellah University, Faculty of Sciences and Techniques, Fez, Morocco. 'Presenting author: said.elkinany@ usmba.ac.ma. \$Corresponding author: rbouamri@ enameknes.ac.ma.

\section{Abstract}

Date palm is an important crop in Morocco, Tunisia and many other drylands of the world, but its growth is often limited due to the low soil fertility and harsh environmental conditions of oases ecosystems, which can hardly be compensated by the sole application of high dosages of chemical fertilizers. For the first time, we investigated the effects of compost application and inoculation with a commercial strain of the arbuscular mycorrhizal fungus (AMF), Glomus iranicum, on the growth of micropropagated date palm plantlets (cv. Feggous). After twelve months of growth, plantlets transplanted into compost amended substrate inoculated with AMF showed increased biomass production (root and shoot biomass), chlorophyll and mineral nutrient contents than plantlets transplanted into compost amended substrate or without compost addition. Thus, this inoculum reinforced the promot- ing effect of compost and was successful in colonizing the root system. According to our results, sandy substrate enriched with compost and inoculated with G. iranicum can be recommended for improving the growth and nutrition of micropropagated date palm plantlets. Keywords: Phoenix dactylifera L., Compost, Mycorrhizal fungi, Organic fertilizer.

\section{P032. Valorization of Natural Resources from Burundi: Case of Myrianthus holstii Engl}

Niyukuri Jonathan" ${ }^{*}$, Jihane Raiti, and Hafidi Abdellatif

Laboratory of Food Sciences of Cadi Ayyad University of Marrakech, Morocco. *Corresponding and Presenting Author: jonaniyu@gmail.com.

\section{Abstract}

The aim of this work is to investigate the chemical composition of the oleaginous seeds of Myrianthus holstii Engl by determining the physicochemical properties of its lipid fraction and the chemical composition of its oilcake. The fruit which is fleshy is edible and a sclerified pericarp surround the seeds which are the object of our study. Samples were harvested in Burundi in three forests following: Vyanda Nature Reserve, Kibira National Park and Bururi Natural Reserve. Analysis were conducted at the Laboratory of Food Science at Cadi Ayyad University in Marrakech, Morocco. The oil extraction is performed with hexane using a soxhhlet apparatus. The obtained yield of oil is $46.58 \pm 6.82 \%$ of Dry Matter (DM), unsaponifiable yield is $1.51 \pm 0.87 \%$, the Oil Acidity is $1.38 \pm$ $0.23 \%$, the lodine Value is $25.37 \pm 6.63 \mathrm{~g}$ iodine / $100 \mathrm{~g}$ of oils and the peroxide value is $5.53 \pm 0.61$ meq / kg of oil. The ethanol $(80 \%)$ extracts was used to analyze the composition of the cake. A Total Polyphenols $0.34 \pm 0.03 \mathrm{~g}$ Gallic Acid Equivalent / 100 g DM was obtained by the Folin-Ciocalteu Method., Sugars content were formed for reach $6.05 \pm 0.37$ g Glucose Equivalent / $100 \mathrm{~g} \mathrm{D} \mathrm{M} \mathrm{and} \mathrm{for} \mathrm{Total} \mathrm{Pro-}$ teins $0.08 \pm 0.01 \mathrm{~g}$ Bovine Alumina Serum Equivalent / $100 \mathrm{~g} \mathrm{DM}$. The results show the potential of use of this species as an oleaginous seed. Nevertheless, this research requires others complementary work for being qualified as safe and edible for human consumption. Keywords: lipid fraction, unsaponifiable. 
P033. The Complementarity of Irrigation Technology Adoption and Groundwater Development; Evidence from the Trifa Plain of Morocco

Aaron R. Hrozencik ${ }^{1 *}$, Emily Burchfield ${ }^{2}$, Dale T. Manning ${ }^{1}$, and Aymen Frija ${ }^{3}$

1 Department of Agricultural and Resource Economics, Colorado State University, Fort Collins, CO 80523, USA $^{2}{ }^{2}$ Department of Environment and Society, Utah State University, Logan, UT 84322, USA; ${ }^{3}$ International Center for Agricultural Research in Dry Areas, Tunis, Tunisia. *Corresponding and Presenting Author: aaronhroz@gmail.com.

\section{Abstract}

A growing world population and changing climate place increasing pressure on agricultural production and already scarce water resources. Promoting irrigation technology adoption and investing in additional sources of water supply are favored policy options to address water scarcity concerns and increase agricultural productivity. While many studies have investigated the effects of irrigation technology adoption on resource use, the literature has largely ignored how technology adoption decisions influence investments in additional water supply and vice-versa. This research addresses that gap in the literature by exploring the interaction between irrigation technology adoption and investments in groundwater development. We utilize remote sensing and survey data from the Trifa Plain of northeastern Morocco to measure the complementarity between sets of irrigation technologies and investments in groundwater pumping capacity. The Trifa traditionally relied on imported water to support the region's robust agricultural economy. However, growing irrigation demand and climatic variability precipitated governmental efforts to promote water conservation and irrigation efficiency through technology adoption, particularly the adoption of on-farm water storage and drip irrigation systems. Imported water deficits also catalyzed increased investment in groundwater pumping infrastructure with the number of irrigation wells in the Trifa increasing by over $46 \%$ between 2000 and 2012 . We leverage the simultaneity of groundwater development and irrigation technology adoption, and the exogenous nature of groundwater availability in the
Trifa to empirically test for complementarity between pumping capacity development and irrigation technology adoption. This work contributes to the literature exploring technology adoption and resource use decisions while also advancing knowledge regarding the impact of investment in groundwater development. Keywords: Groundwater, production economics, agricultural development, technology adoption.

\section{P034. Chemical Composition and Antibacterial Activity of Essential Oil of Artemisia absinthium Against Erwinia amylovora fire blight agent}

Laaziza Doukkali ${ }^{1 *}$, Farthat Guenoun', Bouchra Tazi ${ }^{3}$, and Abdessalem Tahiri ${ }^{2 \S}$

' Laboratory of bioactive molecules and environment, Moulay Ismail University of Sciences B.P 11201 , Meknes, Morocco; ${ }^{2}$ Laboratory of Phytopathology, Department of the Protection of the Plants \& Environment ; National School of Agriculture, BP S 40 Meknès, Morocco; 3 Laboratory of Chemistry, Department of the Basic Sciences; National School of Agriculture, BP S 40 Meknès, Morocco. "Presenting Author: elaazizadoukkali@yahoo.fr. ${ }^{\S}$ Corresponding Author: atahiri@enameknes.ac.ma.

\section{Abstract}

Dealing with resistance and toxicity problems caused by the massive use of plant protection products (pesticides), and with the fact that regulation organizations are questioning the use of antibiotics in agriculture, it is particularly urgent to find alternatives to provide efficient protection of crops against plant diseases. The main objective of this paper is to study the antibacterial activity of essential oil (EO) extracted of Artemisia absinthium against Erwinia amylovora. The extraction was carried out by water distillation Clevenger's type apparatus. The chemical compositions of EO were investigated by using a GC-MS/FID and showed that EO of Artemisia absinthium was characterized by the presence of $\beta$-Thuyone, Camphor and Terpinen-4-ol as the main chemical components. The in vitro antibacterial activity against Erwinia amylovora was evaluated using the method of aromatogram and the method of Microdilution. The results revealed that EO of Artemisia absinthium showed an antibacterial activity against Erwinia Amylovora. Keywords: 
Fire blight, Erwinia amylovora, chemical composition, Antibacterial, activity, chemical, Artemisia absinthium.

\section{P035. Evaluation of Five Mutants of Rapeseed (Brassica napus L.) for Earliness, Agronomic Traits and Response to Changing Environments in M2 Population.}

Souhail Channaoui ${ }^{1,2}$, Mostapha Labhilili ${ }^{3}$, Mohamed Mouhib ${ }^{4}$, Hamid Mazouz ${ }^{2}$, Mohamed El Fechtali ${ }^{1}$, and Abdelghani Nabloussi ${ }^{1 *}$

1 Research Unit of Plant Breeding and Plant Genetic Resources Conservation, National Institute of Agricultural Research, Regional Agricultural Research Center of Meknes, Morocco; ${ }^{2}$ Laboratory of Plant Biotechnology and Molecular Biology, Department of Biology, Faculty of Science, University Moulay Ismail, Morocco; ${ }^{3}$ Research Unit of Plant Biotechnology, National Institute of Agricultural Research, Regional Agricultural Research Center of Rabat, Morocco; ${ }^{4}$ Research Unit of Nuclear Techniques, Environment and Quality, Regional Agricultural Research Center of Tangier, Morocco. *Corresponding and Presenting Author: abdelghani.nabloussi@gmail.com.

\section{Abstract}

Mutagenesis breeding is an efficient strategy to improve rapeseed for several productivity and quality parameters. Five new $\mathrm{M} 2$ mutant populations (H2M1, H2M-2, H2M-3, H2M-4 and H2M-5) were selected from gamma irradiated and Ethyl Methane Sulphonate (EMS) treated material. A field experiment was conducted in two INRA Experimental Stations, at Douyet and Sidi Allal Tazi, to evaluate rapeseed mutants for earliness, agronomic traits and response to changing environments. The variety 'INRA-CZH2' (wild type) was used as a check. Data on days to flowering, days to maturity, plant height, number of branches/ plant, number of pods/plant, number of seeds/pod, pod length, pod diameter and 1000-seed weight were recorded. All the parameters showed significant differences among the mutants studied for both environments. Mutant H2M- 1 was earlier to flowering and maturity and was characterized by a higher number of pods/plant than the check. Mutants H2M-2, H2M4 and $\mathrm{H} 2 \mathrm{M}-5$ produced higher number of branches per plant and 1000-seed weight, compared to the check variety, whilst Mutant H2M-3 exhibited a re- duced plant height and stature. Among the five mutant lines, H2M-1 seemed to be the most interesting and further investigations and trials are needed in order to confirm its performance and stability as a promising and useful germplasm for releasing a new rapeseed variety. Keywords: Rapeseed, mutants, environments, earliness, seed yield.

P036. Genetic Diversity and Variability of the Biochemical Characteristics of Moroccan Loquat (Eryobotria Japonica)

Y. Faiq ${ }^{1,2^{*}}$, H. Hanine ${ }^{1}$, S. Ennahli ${ }^{2}, H$. Latrache', and O. Kodad ${ }^{2}$

1 Laboratory of Bioprocess and Bio-Interfaces, FST, Béni-Mellal, Morocco; ${ }^{2}$ Department of Pomologie, National School of Agriculture Meknes, Morocco. *Corresponding and Presenting Author: Youssef.faiq. cqpaa@gmail.com.

\section{Abstract}

The diversity of climatic conditions gives Morocco a vocation of fruit production in general and fruit rosacea in particular. This sector, which has expanded considerably since the beginning of the 80 s (thanks in particular to private sector initiatives), has a great socio-economic potential and thanks to this important role the new Moroccan agricultural strategy 'The Green Morocco Plan' particular importance to the development of small-scale farming and the promotion of local products. In this aspect, the Green Morocco Plan through projects for the intensification of the loquat in the Zegzel valley and in the surrounding areas has made it possible to improve the quality of Zegzel loquat and to enhance the value of its production through the Protected Geographical Indication. "IGP Loquat of Zegzel". However, the high genetic diversity observed in the loquat orchards of the Berkane area prevents the adoption of an adequate technical itinerary for each cultivar, and consequently a stable quality production from one year to the next. Nowadays, the characterization and evaluation of the diversity and the evaluation of the interannual variability of the physicochemical and biochemical characteristics of the fruit which determine the quality and the specificity of Zegzel loquat within the loquat populations remain unknown. In the perspective of as- 
sessing the diversity of loquat cultivated in Northeast Morocco that the present study was undertaken. The main objective of this study is to study the genotypic variability and to evaluate the biochemical quality of the fruit of the main genotypes selected from the traditional orchards of the Zegzel loquat. A set of 35 individuals were selected and studied. The results of the present study confirmed that the biochemical characteristics of the fruit of the different genotypes of the loquat from the region of Berkane present a wide variability for all the characters measured, they show that some genotypes have presented interesting performances from a quality point of view. fruit. However, the T9 and T11 genotypes have fruits with a high content of carotenoids, flavonoids, and total polyphenols. Keywords: Japanese loquat (Eriobotrya japonica), Biochemical marker, genotype, environment, flavonoids, carotenoids, total polyphenols.

\section{P037. Hepatoprotective effect of methanolic extract of Zygophyllum album against CCl4 -induced hepatotox- icity in rats}

Wassila Saiah", Halima Saiah², Hanane Halzoune ${ }^{3}$, El-Hadi Ahmed Koceir ${ }^{1}$, and Naima Omari ${ }^{1}$

1 Bioenergetics and Intermediary Metabolism, Laboratory, Biology and Organisms, Physiology Department, University of Sciences and Technology Houari Boumediene, Bab Ezzouar, Algeria; ${ }^{2}$ Laboratory of natural bioresources, Faculty of SNV, Department of Biology, University Hassiba Ben Bouali, Algeria. *Corresponding and Presenting Author: saiahbouchra@ yahoo.fr.

\section{Abstract}

Liver diseases pose a major challenge to international public health. Hepatic damage is associated with distortion of metabolic functions of the liver. The present study was carried out to evaluate the hepatoprotective effect of the methanolic extract of Zygophyllum album in CCl4- induced hepatotoxicity in albino rats. Hepatotoxicity was induced in rats by a single intrapéritonéal injection of $\mathrm{CCl} 4$ suspended in olive oil after every $72 \mathrm{~h}$ for 10 days (group II). Hepatotoxicityinduced rats were treated with $100 \mathrm{mg} / \mathrm{kg} \mathrm{b.w.} \mathrm{of}$ methanolic extract of $Z$. album for 10 days, and also, $\mathrm{CCl} 4$ was given as in group II of rats. The effect of the drug was compared with the standard drug silymarin. The effect of the studied extract on antioxidant enzymes, serum glucose, urea, createnine serum bilirubin, total protein and lipid profile was determined. Treatment with Z. album extract showed a significant reduction in blood glucose levels, marker enzymes and lipid profile. The drug significantly increased the activity of antioxidant enzymes in the liver of hepatotoxic rats. The results of the present study suggest that treatment with $Z$. album extract enhanced the recovery from $\mathrm{CCl} 4$-induced hepatic damage due to its antioxidant and hepatoprotective property. Key words: Hepatotoxicity, CCl4, Zygophyllum album, methanol extract, antioxidant.

\section{P038. Pomological evaluation of four mains introduced almond cultivars in North Eastern region of Morocco}

Reda Melhaoui i* ${ }^{*}$, M Addi $^{1}$; M Abid', A Mihamou', H Serghini-Caid ${ }^{1}, M$ Sindic $^{2}$, and A Elamrani ${ }^{1}$

' Laboratoire LBPM, Faculté des Sciences, Université Mohamed ler, Oujda; Morocco; ${ }^{2}$ Laboratoire de Chimie Générale et Organique, Gembloux Agro BioTech, Université de Liège; Belgium. * Corresponding and Presenting Author: reda_melhaoui@hotmail.fr.

\section{Abstract}

Almond (Prunus dulcis L.) is one of the most important cultivated fruit trees in north eastern region of Morocco. Recently, as part of the Green Morocco program, North Eastern region of Morocco was supported by the Belgian development agency through the "PROFAO*" project for planting 6000 ha of almond trees. Ferragnes and Ferraduel, two French cultivars known for their late flowering in March to escape the late frosts of spring, were chosen for the planting of these new almond orchards, instead of "Marcona" and "Fournat" which also have been previously introduced in this region but appears to be sensitive to spring frost due to their flowering in February. This work focuses on the pomological characterization of these four almond cultivars (Ferragnes, Ferraduel, Marcona and Fournat) in five studied areas in North Eastern region of Morocco. For each almond cultivar, recorded pomological measurements for the fruit and kernel are: weight, width, length, thickness, volume and percentage of the double kernels. Nuts weight ranges be- 
tween a minimum value of $2.65 \mathrm{~g}$ for Ferragnes and a maximum value of $4.41 \mathrm{~g}$ for Fournat, while the kernels' weight ranges between $0.73 \mathrm{~g}$ for Marcona and $1.38 \mathrm{~g}$ for Fournat. Calculated mean values of nuts and kernels volumes are respectively $4000.98 \mathrm{~mm} 3$ and $846.09 \mathrm{~mm} 3$ for Marcona, considered as small fruits, and $9667.20 \mathrm{~mm} 3,1553.01 \mathrm{~mm} 3$ for Fournat considered as big fruits in this study, however intermediate values have been recorded for nuts and kernels of Ferragne and Ferraduel. Finally, yield after shelling and the percentage of doubles kernels have been determined, they range from $20.27 \%$ to $33.54 \%$ and from $0 \%$ to $15 \%$ respectively. *PROFAO: Projet Filière Amandes de L'Oriental, "The almond value chain in eastern Morocco, Pillar II of the Moroccan Green Plan, supported by Belgian development agency (BTC 2011-2017). Keywords: Almond, Nut, Kernel, Ferragnes, Ferraduel, Marcona, Fournat.

\section{P039. Distribution of a plant of agronomic interest in Morocco: case of common myrtle}

Aabdousse Jamal ${ }^{*}$, R. Faida, A. Boulli, and N. Wahid ${ }^{\S}$

Ecology and Sustainable Development Team (ESDT), Department of Life Sciences, Faculty of Science and Technology, Sultan Moulay Slimane University, BeniMellal, Morocco. "Presenting author: abdojamal.fstbm@gmail.com. ${ }^{\S}$ Corresponding author: wahid2na@ hotmail.com.

\section{Abstract}

Myrtle is an aromatic and medicinal plant well known for its therapeutic, cosmetic and food properties. In traditional medicine, fruits and leaves are used as antiseptics, antibacterial, antihyperglycemic, analgesic and as anti-inflammatory agent, so in Morocco it is widely used in the control of hair loss and skin toning. These diverse properties make myrtle a plant of interest not only therapeutically but also economically and agronomic. In Morocco, it is exploited from the spontaneous natural state for the extraction of essential oils from leaves. Its uncontrolled exploitation, coupled with the restriction of its natural environment, the degradation of its ecosystem and the reduction of its density within often isolated stations, increase the risk of a rapid genetic erosion of the species. For the sustainable management of this species, it is neces- sary to define a precise vision of the potential uses of myrtle and the prospects of increasing their supply through domestication. Thus, a broad knowledge of the distribution of this species would facilitate the establishment of a seed bank for the development of agricultural domestication and genetic improvement programs. In order to determine the biogeographical range of the Myrtle in Morocco, we have carried out prospecting missions in different regions of the kingdom ranging from the central plateau to the Western Rif. These missions allowed us to determine the bioclimatic stage as well as the vegetation stage of the myrtle, the optimal climatic conditions for the installation, also the domestication and genetic improvement of this species. Keywords: Myrtus communis L., biogeographical distribution, bioclimatic stage, ecology.

\section{P040. Phosphate solubilizing bacteria: Potential Biofer- tilizers for Sustainable Agriculture}

Qessaoui Redouan ${ }^{1,2^{*}}$, R. Bouharroud ${ }^{2}$, A. Amarraque $^{2}$, A. Ajerrar ${ }^{1,2}$, H. Lahmyed ${ }^{1,2}$, E H. Mayad ${ }^{3,4}$, and B. Chebli ${ }^{1}$

1 Laboratoire Génie de l'environnement et de Biotechnologie, Ecole Nationale des Sciences Appliquée, Agadir, Morocco ; 2 Research Unit of Integrated Crop Production, Centre Regional de la Recherche Agronomique d'Agadir (INRA), Morocco; ${ }^{3}$ Laboratory of Biotechnologies and Valorization of Natural Resources Faculty of Sciences - Agadir, Ibn Zohr University, Agadir, Morocco; ${ }^{4}$ Biotechnology and Environmental Engineering Team, Laboratory for Process Environmental and Energy Engineering, National School of Applied Sciences, Ibn Zohr University, PO Box: $1136 / \mathrm{S}$, Agadir, Morocco. *Corresponding and Presenting Author: qessaoui_bio@hotmail.fr.

\section{Abstract}

The concept of sustainable agriculture is a response to the decline in the quality of the natural resource base associated with modern agriculture. The use of chemicals for agricultural needs will result loss in soil physical condition and water pollution. The exploitation of beneficial microbes as a biofertilizers has become paramount importance in agriculture sector for their potential role in food safety and sustainable crop production. Phosphorus is the second mac- 
ronutrient essential for growth and development of plants. However, the solubility of this macronutrient is very low and 75 to $90 \%$ of phosphate fertilizers are precipitated. Two isolates of Pseudomonas (P. putida QEF11B and P. luteola QEF13B) isolated from the rhizosphere were tested in the phosphate solubilization in both solid and liquid media. The $\mathrm{pH}$ evolution was followed. P. putida QEF11B Shows a significant solubilization of phosphate which varies from 0.53 to $2.92 \mu \mathrm{g} / \mathrm{ml}$ for $24 \mathrm{~h}$ and $96 \mathrm{~h}$ respectively. In the solid media each isolate shows a halo around colony. This will be explained by the production of organic acids. The ability of this strain to acidify the media confirmed by the decreased of $\mathrm{pH}$ for both isolates compared to control. To circumvent phosphorus deficiency, this phosphate-solubilizing microorganism could play an important role in supplying phosphate to plants in a more environmentally-friendly and sustainable manner, and reducing problems associated with the use of chemical fertilizers. Keywords: Pseudomonas, phosphate solubilization, Sustainable Agriculture

\section{P041. Responses of differential barley genotypes to Moroccan isolates of Pyrenophora teres f. teres}

Karima Taibi $i^{{ }^{*}}$, F. Bentata ${ }^{2}$, S. Rehman ${ }^{3}$, M. Labhilili ${ }^{2}$, A. El Aissami ${ }^{1}$, R.P.S. Verma ${ }^{3}$, and S. Gyawali ${ }^{3}$

'Laboratory of Botany, Mycology and Environment, Department of Biology, Faculty of

Sciences, University Mohammed V-Agdal, Rabat, Morocco; ${ }^{2}$ L'Institut National de la Recherche Agronomique-Maroc (INRA-M), INRA Avenue Ennasr, Rabat, Maroc BP 415 RP Rabat, Morocco ; ${ }^{3}$ Biodiversity Integrated Gene Management Program (BIGM), International Center for Agricultural Research in Dry Areas (ICARDA), P.O. Box: 6299 Rabat-Instituts/Rabat, Morocco. "Corresponding and Presenting Author: ktaibi1@gmail.com.

\section{Abstract}

Barley net blotch, caused by Pyrenophora teres $f$. teres (Ptt), is one of the most important foliar diseases in Morocco. The disease causes appreciable yield losses under favorable environmental conditions. To identify effective sources of resistance to net blotch, the understanding of virulence spectrum of Ptt is essential. Sixteen barley genotypes were inoculated at seedling stage with $15 \mathrm{Ptt}$ isolates that were collected from different agro-ecological zones of Morocco during the disease survey of 2009-2010. The experiment was conducted in factorial arrangement of treatments in randomized complete block design with three replicates. ANOVA and GGE biplot were employed to understand the barley-Ptt interactions. The ANOVA revealed highly significant effects of genotype (G), isolate (I) and $G \times I$ interactions. Furthermore, the results indicated that race nonspecific interaction and aggressiveness of $\mathrm{Ptt}$ isolates dominated over gene-for-gene interaction (race specific/ virulence). The study of virulence of $\mathrm{Ptt}$ isolates revealed highly diverse virulence pattern of Moroccan isolates. In addition, GGE biplot revealed that Moroccan cultivars, Taffa and Aglou, showed increased level of stable resistance to Ptt comparatively while Coast and Rabat071 were the most susceptible genotypes. The emergence of new Ptt pathotypes which are highly virulent to durable resistance in Rabat071 poses a greater risk of breaking down currently deployed resistance to net blotch in Morocco. GGE Biplot also discriminated virulence of $\mathrm{Ptt}$ isolates of which $\mathrm{P}+2, \mathrm{P}+7, \mathrm{P}+8$, and $\mathrm{P}+4$ being the most virulent isolates, while $P+10$ and $P+11$ were found the least virulent isolates. A careful evaluation and selection of Ptt isolates based on virulence pattern to barley genotypes is essential for successful barley breeding for resistance to net blotch in Morocco. Keywords: Virulence, Barley, GGE Biplot, Net blotch, Pyrenophora teres

P042. Civil development organizations and ICT: two vectors of social change in rural mountain areas of the middle Atlas (morocco)

\section{Noureddine Bahri $^{1^{*}}$, A. Bentaïbi ${ }^{1}$ and T. Desrues ${ }^{2}$}

1 Regional Center of Agricultural Research of Meknes, BP. 578 Meknes VN, Morocco; ${ }^{2}$ Institute of Advanced Social Studies (IESA) Cordoba, C/ Campo Santo de los Mártires, 714004 Cordoba, Spain. "Corresponding and Presenting Author: bahrinour@yahoo.fr.

\section{Abstract}

The positioning of civil organizations (NGOs) as an important stakeholder within the institutional landscape for local development is now recognized as a real characteristic of the new development dynamics 
that Morocco is experiencing during the last two decades. Local development associations and cooperatives are a tool for mobilizing the population around concrete projects and a key partner for their participatory realization. They are also a local anchor of sustainability and scalability capable of assimilating the social demand and transforming it into innovative initiatives for the production of products and services. Such organizations finally provide the advantage of having a broad credit to the various actors and are therefore able to play an integrative role for the various sectoral development interventions. Access to information and communication technologies (ICTs) is a phenomenon illustrating the dynamics of change recorded in the Kingdom and is considered as another vector of social change. As a result, NGO's access to ICTs would allow: 1) to break their isolation, fostering internal and external communications; 2) to provide information on local development strategies and needs; 3) To obtain resources and establish partnerships or raise awareness among policy and administrative leaders; and 4) to promote the participation of their members and local people. These hypotheses are supported by the results of our study, ICTs are, however, resources or instruments that are part of a pre-existing social, economic and political order, so it is appropriate to check in accordance with each local situation the current reconfigurations and whether the latter promote the reproduction of the old positions of power and inequality or are, on the contrary, sources of change. We analyse also the ICTs and development actions carried out by the three selected NGOs. Secondly, we deepen the question of the place of women in the activities and decision-making processes of these NGOs and, in particular, we return to the empowerment opportunities that ICTs offer to women. In conclusion, we present some of the development opportunities offered by ICTs and certain identified limits, especially in terms of capacity building and gender. Keyword: ICTs, NGOs, Local development, Agricultural development, Collective action, Social change.

P043. Characterization and phylogenetic diversity of Allorhizobium vitis isolated from grapevine in Morocco

Khaoula Habbadi ${ }^{1,2,3^{*}}$, Quentin Duplay ${ }^{3 *}$, David Chapulliot $^{3}$, Isabelle Kerzon ${ }^{3}$, Rachid Benkirane ${ }^{2}$, Abdellatif Benbouazza', Florence Wisniewski-Dye ${ }^{3}$, Céline
Lavire $^{3}$, Ludovic Vial ${ }^{3}$, and El Hassan Achbani ${ }^{1}$

${ }^{1}$ Laboratoire de recherche et de protection des plantes URPP-INRA-Meknès, Morocco; ${ }^{2}$ Laboratoire de Botanique, Biotechnologie, et Protection des Plantes, Faculté des Sciences, Kenitra, Morocco; ${ }^{3}$ Université de Lyon, Université Claude Bernard Lyon ', CNRS, UMR 5557, Ecologie Microbienne, INRA, UMR 1418 , 10 Villeurbanne, F-69622, France. "Corresponding and Presenting Author: khaoula405@gmail.com.

\section{Abstract}

Crown gall was observed in recently planted vineyards of the Meknes region (Morocco). 82 isolates from 10 affected vineyards were characterized by recA sequencing and were found to belong to $A$. tumefaciens genomospecies G1, G4 or G7, Rhizobium rhizogenes, and to Allorhizobium vitis. Only the 39 A. vitis isolates showed to be pathogenic in vitro on tomato and MLSA phylogenetic analyses revealed a poor genetic diversity, with the definition of only four genomic groups. Definition of the A. vitis genomic groups correlates with specific pathogenic traits: indeed, genomic groups differ with respect to the severity of hypersensitive response symptoms on tobacco leaves, the intensity of necrotic response on grapevine explants, and opine profiles. Both vitopine and octopine were detected by UHPLC in tumors induced by isolates of three genomic groups, an opine signature scarcely ever reported. Keywords: Crown gall, grapevine, Allorhizobium vitis, Agrobacterium tumefaciens, phylogenetic diversity.

\section{P044. Biological Control of Bacterial Onion Diseases using a Bacterium, Pantoea agglomerans 2066-7}

Soumia Sadik ${ }^{1,2^{*}}$, Hamid Mazouz ${ }^{2}$, Abdellatif Benbouazza ${ }^{1}$, and El Hassan Achbani ${ }^{1}$

1 Laboratory of Phytobacteriology and Biocontrol, National Institute of Agronomic Research, BP. 578Meknès VN 50000, Km 13 Route Haj Kaddour, MeknesMorocco; ${ }^{2}$ Laboratory of Plant Biotechnology and Molecular Biology, Faculty of Sciences, b p 11201 , Avenue Zitoune, Meknes, Morocco. "Corresponding and Presenting Author: sadik.soumia@hotmail.com 


\section{Abstract}

Epiphytic microorganisms, isolated from the olive knots, apple fruits and trees, quince, compost and water from different areas were screened for antagonistic activity against Pseudomonas marginalis, Pseudomonas viridiflava, Xanthomonas retroflexus and Pantoea ananatis on onion bulbs. From 77 microorganisms tested for antagonistic property against bacterial onion diseases, the strain 2066-7 of Pantoea agglomerans was selected. Complete control against Pseudomonas marginalis and Pseudomonas viridiflava at $107 C F U$. $\mathrm{ml}-1$ concentration of 2066-7 and an inhibition percent higher than $90 \%$ against Xanthomonas retroflexus and Pantoea ananatis were obtained on wounded onion bulbs inoculated with 106CFU.ml- 1 of pathogens under cold conditions. The inhibitions percent were decreased under $25^{\circ} \mathrm{C}$ and $30^{\circ} \mathrm{C}$. Keywords: Onion, Bacterial onion diseases, Storage, Biological control, Morocco

P045. Effect of Parturition and Lactation on Ponderal Performance in Bedouin Goat under Harsh Climatic Environment

Henna Kamilia, Kouri Fatima, Kouri Amina, Amirat Zaina, Khammar Farida, and Charallah Salima ${ }^{*}$

Research Laboratory of Arid Lands, Faculty of Biology, USTHB, BP 32 El-Alia, Algiers, Algeria. "Corresponding and Presenting Author: charalla@yahoo.fr.

\section{Abstract}

In various animal species, many studies have shown weight fluctuations during parturition and lactation, however, no ponderal study was carried out during these physiological stages in the native bedouin goat in the Algerian Sahara. Given its perfect adaptation to the hostile conditions of the arid zones, it seemed interesting to describe the evolution of body weight (BW) during these critical phases. This study is conducted on 14 females kept at the research station of Beni-Abbes (south- west Algeria: $30^{\circ} 07^{\prime} \mathrm{N}, 2^{\circ}$ $\left.10^{\prime} \mathrm{W}\right)$. These females are weighed in the morning, on the day of parturition (DO) and weekly for six weeks post-partum (W1 to W6). The weight data obtained are analyzed by statistical tests Kruskal Wallis and Dunn (XL Stat). the mean BW on day 0 is $23.2 \pm 5.4 \mathrm{~kg}$ but becomes significant from $W 2$ to W5 and drops very significantly in W6, respectively $(20.5 \pm 5.1,20.2 \pm 5.1,19.9 \pm 3.9$ et $19.2 \pm 4.00 \mathrm{~kg}$, $p=0.01 ; 18.9 \pm 3.1 \mathrm{~kg}, p=0.006)$. The statistical difference between mean weekly BW from W1 to W6 is not significant. The weight losses recorded during lactation would indicate the low level of ingestion capacity leading to an energy deficit that the lactating female must compensate by using her body reserves accumulated during the second half of the gestation period. At the same time, a progressive average daily gain is noted in newborns. The average BW in DO would indicate the accumulation of lipid reserves during pregnancy and nutritional quality ingested. These weight variations reflect the interaction between the food supply and the mobilization of body reserves according to the physiological stage. Keywords: Parturition, lactation, ponderal performance, goat, arid zone.

P046. In vitro evaluation of the effectiveness of peel pomegranate aqueous extract in controlling Brown rot caused by Monilinia spp.

Assia Elkhetabi ${ }^{1,2 *}$, Rachid Lahlali', Said Ezrai ${ }^{1,2}$, Abdessalem Tahiri', Latifa Askarne ${ }^{3}$, Jovana Hrustic ${ }^{4}$, Lahsen El Ghadaroui ${ }^{2}$, and Said Amiri

1 Department of Plant Protection, Phytopathology Unit, Ecole Nationale d'Agriculture de Meknès, BPS 40, Meknès, Morocco; ${ }^{2}$ Laboratory of Functional Ecology and Environment, Sidi Mohamed Ben Abdellah University, Po. Box 2202, Route d'Imouzzer, Fez, Morocco; 3 Laboratory of Microbial Biotechnology and Plant Protection, Université Ibn Zhor, Faculté des Sciences, Agadir, Morocco; 4 Institute of Pesticides and Environmental Protection, Laboratory of Applied Phytopathology, Banatska 31 b, 11080 Belgrade-Zemun, Serbia. "Corresponding and Presenting Author: elkhetabiassia@gmail.com.

\section{Abstract}

Brown rot is considered as one of major pre-and postharvest pathogen of tree fruit and it is commonly caused by both fungal pathogens Monilinia laxa and M. fructigena. This disease was usually controlled based on integrated strategy, which includes cultural practices and fungicide spray. However, due 
to environmental and consumers health concerns, alternatives strategies need to be developed in order to substitute the frequent use of chemical substances. Therefore, this study aimed at evaluating the in vitro antifungal activity of peel pomegranate aqueous extract against both fungal pathogens causing brown rot. The preliminary results showed a promising effect of the peel pomegranate aqueous extract on the in vitro mycelial growth of both pathogens when tested at $2.5 \mathrm{mg} / \mathrm{ml}$ of PDA medium. In addition, obtained results highlighted a positive correlation between the concentration rate of peel pomegranate aqueous extract and the mycelial growth inhibition. Interestingly, the inhibition rate was increased with increasing the concentration rate of peel pomegranate aqueous extract from $0.5 \mathrm{mg}$ to $5 \mathrm{mg} / \mathrm{ml}$. It was concluded that the aqueous extract of peel pomegranate might be a sustainable alternative control strategy of brown rot. As a result, studies on fruit and mechanisms behind its effectiveness are further considered in more details. Keywords: Brown rot, tree fruit, alternative control strategy, aqueous extract, peel pomegranate.

\section{P047. Survey and prevalence of viruses infecting cucur- bit crops in Morocco}

Nabil Radouane ${ }^{1,2^{*}}$, Soukaina Ermadi ${ }^{1}$, Abdessalem Tahiri $^{i^{*}}{ }^{*}$ Meryem Benjelloun ${ }^{2}$, and Rachid Lahlali ${ }^{1}$

1 Department of Plant Protection, Phytopathology Unit, Ecole Nationale d'Agriculture de Meknès, BPS 40, Meknès, Morocco; ${ }^{2}$ Laboratory of Functional Ecology and Environment, Sidi Mohamed Ben Abdellah University, PO Box 2202, Route d'Imouzzer, Fez, Morocco. "Corresponding and Presenting Author: atahiri@enameknes.ac.ma.

\section{Abstract}

Cucurbit species are important vegetable crops in Morocco and many Mediterranean countries. A survey carried out within 2016 and 2017 in open field has emphasized severe yellowing, chlorosis, and symptoms of viral infections in major cucurbits growing areas of Morocco; in particular Azmour, El Jadida, Kenitra, Taroudant, Zagoura, and Agadir. Accordingly, samples having virus symptoms in two cucurbits corps Zucchini (Cucurbita pepo) and Watermelon (Citrulus lanatus) were collected, dried with calcium chloride, and analyzed by DAS-ELISA technique for detection of the most common cucurbit viruses such as Cucumber mosaic virus (CMV), Zucchini yellow mosaic virus (ZYMV), Water melon mosaic virus (WMV), Papaya ringspot virus (PRSV) and Cucurbit aphid born yellow virus (CABYV). In addition, both PCR and RTPCR were used for specific detection of Tomato leaf curl New Delhi virus (ToLCNDV), Cucumber mosaic virus (CMV), Cucumber vein yellow virus (CVYV), and Melon necrotic spot virus (MNSV). Among 136 cucurbit samples (86 samples of zucchini and 50 samples of watermelon), the DAS-ELISA revealed that $79.07 \%$ of the zucchini samples and $60 \%$ of the watermelon samples were positive for ZYMV, WMV, PRSV and CABYV. Surprisingly, the PCR bioassay revealed the presence of ToLCNDV for the first time in the country. Regardless of the locality, the CMV was detected only by RT-PCR in most samples, while the CVYV and the MNSV were absents. Keywords: Cucurbita pepo, Citrulus lanatus, CMV, ZYMV, PRSV, CABYV, CMV, CVYV, MNSV, ToLCNDV, DAS-ELISA, PCR and RT-PCR.

P048. Effect of Silicon Application on Seed Germination of Coriandrum sativum (L.) Grown Under Salt Conditions

Jamila Fahimi ${ }^{1,2^{*}}$, Fouad Achemchem 1 , Zakia Bouzoubaâㄹ , Nabil Saffaj', Rachid Bouharroud ${ }^{2}$, and Rachid Mamouni ${ }^{1}$

1 Team of Materials, Catalysis and Natural Resources Development, Department of Chemistry, Ibn-Zohr University, FSA. Agadir, Morocco; ${ }^{2}$ Agrophysiology \& Post Harvest Laboratory Natural Resources and Local Product Research Unity (UR RN \&PDT) INRA-CRRAAgadir, Morocco. *Corresponding and Presenting Author: jamila.fahimi@edu.uiz.ac.ma/f.achemchem@ viz.ac.ma.

\section{Abstract}

Salinity is one of the main factors limiting the growth of plants. Agricultural losses caused by salinity are alarming and in continuous increase. Silicon $(\mathrm{Si})$ is the second abundant element in the earth's crust, performing a great role in healing plants in response to abiotic stresses. Seed germination is critical step of the plant development. For that, this work aims to evaluate the germinative behaviour of coriander $\mathrm{Co}$ - 
riandrum sativum (L.) in salt stress conditions in presence and absence of $\mathrm{Si}$. Seeds were germinated in Petri dishes in three levels of salinity $(0,25 \mathrm{mM}$ and $50 \mathrm{mM}$ of $\mathrm{NaCl}$ ) with and without Si supply, the number of seeds germinated was recorded daily for 10 days. Our results exhibit that salt treatments acted negatively on germination percentage (\%), coefficient of velocity of germination (CVG), germination rate index (GRI) and mean germination time (MGT). Whereas, the application of Si enhanced these germination indexes. Keywords: Silicon; Salinity; Coriandrum sativum (L.); Germination.

P049. Improvement of tomato rhizobial inoculant by incorporation of plant growth promoting rhizobacteria (PGPR) as biocontrol against the bacterial canker caused by Clavibacter michiganensis subsp. Michiganensis

Meryam Moustaine ${ }^{1,2^{*}}$, R. Elkahkahi ${ }^{3}$, A. Benbouazza ${ }^{1}$, R. Benkirane ${ }^{2}$, and El Hassan Achbani ${ }^{1}$

Laboratory of Bacteriology and Biological Control, the National Institute of Agronomic Research of Meknes. Morocco; ${ }^{2}$ Laboratory of Botany and Plant Protection, Faculty of Sciences, University Ibn Tufail, Kenitra, Morocco; ${ }^{3}$ Centre for Innovation and Technology Transfer, University Moulay Ismail, Marjane 2, Meknes. Morocco. *Corresponding and Presenting Author:meryamenam@gmail.com.

\section{Abstract}

Three bacterial strains from the compost were tested for their antagonistic properties against the pathogenic bacterium Clavibacter michiganensis on tomato. Their efficacy was compared with that of the Pantoea agglomerans bacterium (2066-7) whose ability to control Clavibacter michiganensis is recognized. The results show that Pantoea agglomerans reduces the onset of $70 \%$ disease symptoms, Serratia proteamaculans to $45 \%$ and Bacillus cereus to $75 \%$. Subsequent trials will verify the antagonistic efficacy of the combination of these three rhizobacteria on Clavibacter michiganensis. Keywords: Clavibacter michiganensis, rhizobacteria, antagonistic.
P050. Study of Several Physicochemical Properties of four Sweet Cherry Cultivars (Prunus avium L.) in the Middle Atlas Mountains of Morocco

Mina El Baii ${ }^{12^{*}}$, H. Hanine ${ }^{2}$, H. Latrache ${ }^{2}$, and O. Ko$\mathrm{dad}^{1}$

1 Department of Pomologie, National School of Agriculture- Meknes, Morocco; ${ }^{2}$ Laboratory of Bioprocess and Bio-Interfaces, FST Béni-Mellal, Beni Mellal, Morocco. "Corresponding and Presenting Author: m.elbaji92@gmail.com.

\section{Abstract}

Fruit samples analyzed in this study were harvested in 2015 and 2016 from four sweet cherry cultivars, namely "Burlat", "Van", "Napoleon" and "Pigeon heart" grown in the Middle Atlas of Morocco (Toufselt and Imouzzer). Some physicochemical properties of fruit were investigated. Fruit weight ranged from $6.39 \mathrm{~g}$ and $6.90 \mathrm{~g}$, but statistical differences between "Burlat", "van" and "Pigeon heart" were non-significant. Fruit volume in different cultivars was between 4.89 and $5.26 \mathrm{~cm} 3$. The highest values were recorded in "Van" and "Pigeon heart". The lowest values of flesh width were in cultivars "Burlat" (7.26 $\mathrm{mm})$ and "Napoleon" (7.12 mm). The epidermis of "Napoleon" cherries had low firmness, whereas high firmness values were measured in "Van" and "Pigeon heart". Titratable acidity was between 8.36 and $11.05 \mathrm{~g}$ malic acid/l, "Napoleon" having the highest value. Soluble solids content in different cultivars was between $14.5^{\circ}$ Brix and $16.07^{\circ}$ Brix. The highest value was recorded in "Van". Fruit weight and firmness were higher in 2016 than in 2015 while fruit length and thickness were lower. "Toufselt" location shows high values of fruit weight and size. The fruits produced in "Imouzzer" locations are characterized by a high firmness. Keywords: sweet cherry, cultivar, physicochemical properties.

P051. Humics substances produced during composting process of the olive mill wastes and municipal organic wastes

Barje Farid ${ }^{*}$ and Hafidi Mohamed

Laboratory of Ecology and Environment, Faculty of 
Science Semlalia, Cadi Ayyad University, Marrakesh, Morocco. "Corresponding and Presenting Author: fbarje@gmail.com.

\section{Abstract}

The humics substances form an important fraction of soils' organic compounds; their chemical nature has put forward various hypotheses on the mechanisms of their formation. In this context, to follow their evolution, the Humic acids(HAs) and Fulvicacids (FAs) extracted at different stages of co-composting of olive mill waste and organic household refuse with added phosphate, were analyzed with 13C-NMR and FT-IR spectroscopy. The findings highlight the contrasting variations in theproportions of $\mathrm{C}$-aromatic and $\mathrm{C}$-carbonyl of HAs as the $\mathrm{H} / \mathrm{C}$ atomic ratio changes. The comparative 13C-NMR study of the FAs with the same tendancy, that showlow aromatic $(C=C)$ content in the initial stage of composting, which contained a greater proportion of carbonyl $(C=O), O, N$-alkyl and alkyl carbon. The FT-IR spectroscopy of HAs showed a decreasingabsorption in bands of aliphatic bonds - $\mathrm{CH}-,-\mathrm{CH} 2-$, carboxylic acids, esters, amides $\mathrm{C}=\mathrm{O}$, the $\mathrm{C}-\mathrm{O}$-bonds of polysaccharides and alcohols with respect to ether bonds $\mathrm{C}-\mathrm{O}-\mathrm{C}$; they was linearlycorrelated with the $\mathrm{H} / \mathrm{C}$ ratio. For the FAs, the main absorption bands were in the region of 3393 $\mathrm{cm}-1(\mathrm{O}-\mathrm{H})$, near $2931 \mathrm{~cm}-1$ (aliphatic $\mathrm{C}-\mathrm{H})$, a distinct shoulder between $1717 \mathrm{~cm}-1$ ( $\mathrm{C}=\mathrm{O}$ of carbonyl) and $1640 \mathrm{~cm}-1$ ( $C=O$ of amide and ketone), 1547 $\mathrm{cm}-1$ (aromatic $\mathrm{C}=\mathrm{C}$ with conjugated $\mathrm{C}=\mathrm{O}$ or amide N-H), near 1400-1384 cm-1 ( COO- stretch or $\mathrm{OH}$ deformation of $\mathrm{COOH}$ ). The ratio of $\mathrm{HAs}$ to FAs carbon content (CHA/CFA), which is representative of the polymerization degree, showed a positive linear correlation $(r=+0.56, p<0.05)$ with the degree of aromaticity of HAs. The increase in the CHA/CFA ratio with increasing humic acidsaromaticity, is representative ofa remarkable aspect of the restructuring brought, with respect to the mechanisms ofpolymerization during composting. Keywords: Humic Acids, Fulvic Acids, Composts, Organic wastes.

P052. Physicochemical characterization of the pomegranate (Punica granatum) in INRA collection: a first step of selection of performants cultivars

Assia Eiiilani ${ }^{1,2^{*}}$, Hafida Haninie ${ }^{2}$, Rachid Razouk ${ }^{1}$, La- hcen Hssaini', Abderrahman Mekaoui', Hakim Outghouliast ${ }^{1}$, and Jamal Charafi ${ }^{1 \S}$

1 National Institute of Agricultural Research (INRA), regional center of Meknes, Morocco; 2 Laboratory of Bioprocess and Bio-Interfaces, Faculty of Science and Technology, Beni Mellal, Morocco. " Corresponding and Presenting Author: assiaejiila@gmail.com. §Corresponding author: jcharafi@gmail.com.

\section{Abstract}

In order to support the development of the pomegranate (Punica granatum) in Morocco, a collection of several important varieties has been installed in experimental station of the National Institute of Agricultural Research (INRA) located in Ain Taoujdate. This study was carried to assess the appropriate physicochemical compounds of fruit for a future selection of performing varieties for agriculture and industry. Eighteen cultivars were characterized including 10 local genotypes and 8 foreigners. Measurements concerned parameters related to the external, internal and qualitative characteristics of fruit (weight, dimensions, weight of arils, pips, brix degree of juice, ...). Our results show a large diversity based on the used descriptors. For fruit weight, it varies between $198.14 \mathrm{~g}$ for local cultivar "Chelf" and $589.28 \mathrm{~g}$ for foreign cultivar "Red Pomegranate 2". The fruit length differs between 58.93 (Chelfi) to $96.3 \mathrm{~mm}$ (Red Grenade2) and its diameter between 72.29 (Chelfi) and $109.99 \mathrm{~mm}$ (Red Pomegranate 2). Total arils weight varies between 127 and $463 \mathrm{~g}$ with an average of $237 \mathrm{~g}$. Thus, the weight of one aril varies from 2.6 to $8.9 \mathrm{~g}$ with an average of 4.3. Fruit juice content reached from 25 to $52 \%$. The high values of juice content were observed for cultivars Zheri of Autumn', Zheri Precoce', and 'Red Pomegranate', thus presenting an interest in industry. The flavor and the hardness of arils have a great influence on fruit taste quality. The cultivars Red Pomegranate, Sefri and Yellow Pomegranate have a good quality marked by a sweet taste (17-20 ${ }^{\circ}$ Brix), a high richness in anthocyanins and a softness of their pips. These primary results show that this ex-situ collection of pomegranate contains a large physicochemical diversity and can be a basis for selecting the performing varieties for different regions. Keywords: Pomegranate, Punica granatum, characterization, physicochemical, quality, 
ex-situ collection

\section{P053. Valorization of the Therapeutic Virtues of Salvia mouretii Extracts from Atlas Mountains of Morocco}

Sanae Jennan ${ }^{1 *}$, Abdellah Farah², Fatima Mahjoubi ${ }^{1}$, and Dalila Bousta ${ }^{3}$

1 Materials Engineering and Environnement Lab, Department of Chemistry Sciences, Sidi Mohamed Ben Abdellah University, Faculty of Sciences Dhar El Mahraz, BP 1796 Fez, Morocco; ${ }^{2}$ Applied Organic Chemistry Lab, Department of Chemistry, Faculty of Sciences and Techniques, Fez, Morocco; 3 Régulations Neuroendocriniennes liées à l'environnement Lab, Faculty of Sciences Dhar El Mahraz, BP 1796 Fez, Morocco. *Corresponding and Presenting Author: jennansanae@gmail.com.

\section{Abstract}

This study aims to investigate in vitro antioxidant activities of Salvia mouretii and to examine the in vivo antiinflammatory and antinociceptive effects of the aqueous extract of aerial parts of S.mouretii. The aqueous and ethanol extracts were screened for their possible antioxidant activities by three complementary test systems, namely DPPH free radical-scavenging, reducing power and molybdenum systems. Anti-inflammatory activity of the aqueous extract was evaluated by CarrageenanInduced Rat Paw Oedema method. The antinociceptive effect was tested by using the plantar test. The ethanol extract showed better activity (IC50 $=0.4 \mathrm{mg}$ ) $\mathrm{ml}$ ) in the DPPH system. The aqueous extract showed greater activity in the phosphomolybdenum method (389.75 equivalent to ascorbic acid $\mathrm{mg} / \mathrm{g}$ of extract and 188.73 equivalent to BHT $\mathrm{mg} / \mathrm{g}$ of extract) and exhibited the highest reducing power. The aqueous extract of S. mouretii at 10\% inhibited the inflammation induced by carrageenan in rats at $75 \%$ compared to $67.5 \%$ for diclofenac at $1 \%$ after $4 \mathrm{~h}$ of inflammation induction. However, at dose of $20 \%$, S. mouretii showed a pro-inflammatory effect. In the plantar test, the tolerance time of the rats treated with $10 \%$ of the aqueous extract was significantly higher compared to the control group. These initial results tend to support the traditional use of S. mouretti used in Morocco for the treatment of burns. Keywords: Salvia mouretii, Antioxidant activity, Anti-inflammatory activity, antinociceptive activity.
P054. Characterization of Rhizobacteria Isolated from Compost and their Potential Effect on Promoting Tomato (Solanum lycopersicum L.) Growth

M. Moustaine ${ }^{1,2^{*}}$, R. Elkahkahi ${ }^{3}$, A. Benbouazza ${ }^{1}$, R. Benkirane $^{2}$, and El Hassan Achbani ${ }^{1}$

' Laboratory of Bacteriology and Biological Control, the National Institute of Agronomic Research, Meknes, Morocco; ${ }^{2}$ Laboratory of Botany and Plant Protection, Faculty of Kenitra Ibn Tufail Sciences, Kenitra, Morocco; ${ }^{3}$ Centre for Innovation and Technology Transfer, University Moulay Ismail, Marjane 2, Meknes. Morocco. "Corresponding and Presenting Author: meryamenam@gmail.com.

\section{Abstract}

Rhizobacteria promote plant growth (PGPR) are soil bacteria which colonize roots of the plant, improve growth and live symbiotically with it. These microorganisms can promote plant growth by nutritional and hormonal balance control, production of plant growth regulators, the solubilization of nutrients (such as phosphate) and including resistance against plant pathogens. The objective of this work was to study the PGP activities and production of antimicrobial enzymes of three bacterial strains belonging to the genus Bacillus cereus (2027-2), Pantoea agglomerans (2066-7) and Serratia proteamaculans (2025-1), which are promoter's rhizobacteria plant growth and having been tested on the culture of the tomato field. Only the strains 2066-7 solubilize the phosphate in the culture medium PVK. Although all isolates produce IAA 2066-7 product the high concentrations of this strain phytohormone in addition to its ability to synthesize all antimicrobial enzymes. All isolates showed an acetic acid indole activity in large amounts when cultured in the presence of tryptophan precursor to 1 $\mathrm{g} / \mathrm{I}$. This characterization has allowed to know the attributes benefits, which account for the benefits of competitiveness of these bacteria in the rhizosphere of crops, which, with their ability to perform biological nitrogen fixation, could promote the growth of tomato plants. Keywords: PGPR, AIA, solubilization phosphate, nitrogen fixation, production of ammonia (NH3) and Production antimicrobial enzymes. 
P055. Conservation of Moroccan saffron with molecular identification by microsatellite markers (Crocus sativus L.)

Mohamed Lachheb-", M. Ben El Caid, L. Salaka, K. Lagram, Mohamed Amine Serghini, and Abdelhamid El Mousadik

Laboratory of Biotechnology and Valorization of the Natural resources (LBVRN), Department of Biology, Faculty of Science, University lbn Zohr, Agadir, Morocco. "Corresponding and Presenting Author: lachheb.mohamed91@gmail.com.

\section{Abstract}

Crocus sativus $L$. is a male sterile vegetatively propagated plant. Its flower produces stigmas that when dried, constitute the most expensive spice in the world commonly known as saffron' Red Gold', the name saffron is usually used to refer toboth the spice and the plant itself. This plant is cultivatedin anumber ofcountries in the world (Iran, Turkey, India, Greece, Morocco, etc.). In Morocco, saffron is primarily cultivated in the area of Taliouine/Taznakht. The Moroccan Saffron is very popular at both national and international level. The Conservation and enhancement of the Moroccan saffron impose their morphological, biochemical and molecular characterization. The present study has for objectives, the development of a molecular identity of Moroccan saffron, the investigation and evaluation of the genetic variability by molecular markers and the construction of a database allowing fora better management, selection, exploitation and conservation of the Moroccan saffron as anational heritage. The samples used in this study, were collected from four different selected areas (provenances) in the region of Taliouine/Taznakht (Sidi Hssaine, Agadir Melloul, Taznakht and Askaouen) with an average of four samples of leaves from each provenance. Concerning the genomic DNA, it was extracted from $40 \mathrm{mg}$ of leaves, using $C T A B$ procedure and it has served as the matrix for the amplification by PCR. The microsatellite markers were selected for the identification and analysis of the genetic variability of cultivars of the Moroccan saffron, because of their large polymorphism, their specificity, their codominance, their reproducibility and stability. 359 EST sequences of genomic DNA of the saffron were collected from the
National center for Biotechnology Information (CNIB). The SSRIT software has allowed the identification of 14 EST-SSR (SSRe) and 12 SSR from the genomic DNA (SSRg), the primers specific for each region SSR were determined by the software Primer 3 version 4.0.0. In addition, 12 pairs of primers developed by Nemati et al. (2012) among the Iranian Saffron and three pairs of primers determined at Iris ensata, were selected for the molecular characterization of the Moroccan Saffron. The first results show that Moroccan Saffron is genetically different compared to the Iranian saffron, this data is promoter for developing of a conservation strategy for the Moroccan saffron. The provenance of Sidi Hssaine is a clone genetically and agro-morphologically different from other provenances, this data is in favor of a high potential for the improvement of the productivity of the saffron of Taliouine with a selection assisted with SSR markers. Keywords: Microsatellite, saffron (crocus sativus), molecular markers, genetic diversity, molecular identity.

\section{P056. Phytochemical and antibacterial study of Thymus zygisin the Azrou region}

Fatima Zahrae Radi ${ }^{1,2^{*}}$, Sanae Amine ${ }^{1,2}$, Mohamed Regragui ${ }^{1,2}$, Najia Hamzaoui ${ }^{1,2}$, Hamid Oulhaj ${ }^{1,2}$, and Touriya Zair ${ }^{1,2}$

1 Research team in the chemistry of bioactive molecules and environment. Department of Chemistry. Moulay Ismail University, Faculty of Sciences, PO Box 11201,50000 Zitoune Meknès, Morocco; ${ }^{2}$ Laboratories of Materials Chemistry and Biotechnology of Natural Products (Chima-Bio).Department of Chemistry. Moulay Ismail University, Faculty of Sciences, PO Box 11201,50000 Zitoune Meknès, Morocco. "Corresponding and Presenting Author: fati_radi2007@ hotmail.com.

\section{Abstract}

As a part of the valuation of the Moroccan cultural heritage in aromatic and medicinal plants, a phytochemical and antibacterial study of a species widely used in traditional medicine was carried out, it is the thymus zygis of the Azrou region. This plant's yield of essential oil extracted by hydrodistillation is of the order of $5.25 \pm 0,01$. The essential oil (EO) was analyzed using gas chromatography coupled with 
mass pectrometry (GC-MS). It contains as majority compounds: the thymol $(32.46 \%)$, and the carvacrole $(18.29 \%)$. This plant has undergone a phytochemical screening which consists of detecting the different chemical compounds existing in the plant using precipitation and coloring reactions. Thus, it is rich in tannins, flavonoids, sterols and triterpenes, and free from the alkaloids of coumarins, and mucilage. For the antibacterial activity two methods were used: The first one is the method of diffusion on the disk and the second one is the method of the Minimal Inhibitory Concentration (MIC) and the Minimum Bactericidal Concentration ( $C M B)$. The essential oil was tested against six bacterial species: Escherichia coli, Staphylococcus aureus, Salmonella typhi, Acinetobacter baumanii, Shigella dysenteria, and enterobacter cloacae. The results obtained showed that the plant's essential oil inhibited the activity of all the species at low volumes $(2 \mu \mathrm{l} / \mathrm{ml})$. The CMB was identical to the $\operatorname{MIC}(2 \mu \mathrm{l} /$ $\mathrm{ml}$ ). According to the results of this study, we conclude that this medicinal plant possesses very important biological properties which can be widely used in various fields such as medicine, pharmacy, cosmetology and agriculture. Keywords: Aromatic and medicinal plants, Thymus zygis, phytochemical study, Bacterial species, essential oil and antimicrobial power.

\section{P057. Numerical simulation of the airflow, temperature and humidity fields distribution inside multi-span green- house equipped with insect proof under different out- side climate conditions}

$\underline{\text { Reda Errais }}^{{ }^{2}}{ }^{*}$, Ahmed Bekkaoui ${ }^{1}$, Hassan Majdoubi ${ }^{2,3}$, Younes El Fellah', and Allal Senhaji ${ }^{3}$

1 Département Energie et Agroéquipements. Institut Agronomique et Vétérinaire Hassan II, Rabat, Morocco; ${ }^{2}$ Laboratoire de Recherche Scientifique et Développement Pédagogique. CRMEF, Fès-Meknès, Morocco; Equipe de Recherche en Energétique et Mécanique des Fluides. Ecole Nationale Supérieure d'Arts et Métiers, Meknès, Morocco. "Corresponding and Presenting Author: Redaerrais@gmail.com.

\section{Abstract}

The agricultural greenhouses are structures that create a favorable and optimal microclimate adapted for the growth and the development of each crop by controlling the climate and by reducing the introduction of the various insects harmful to plants. Air circulation, temperature and humidity are the most important parameters of greenhouse climate that needs to be controlled in order to achieve optimal plants growth and maximize the yield. The aim of the current study is to predict the distributed climate inside multi-span tomato-greenhouses equipped with fine insect proof in sidewalls ventilation openings. Also, we try to analyze the greenhouse ventilation efficiency under two different external climate conditions in order to determine the effect of the outside weather conditions on the airflow, temperature and humidity fields distribution inside and at the greenhouses openings. For this purpose, a 3D numerical simulation using a Computational Fluid Dynamics (CFD) was carried out to estimate the dynamic and thermal and humidity behavior by using a commercial software package Fluent v6.3.26 based on the finite volumes method to solve the mass, momentum and energy conservation equations. The turbulent transfers were described by model. Likewise, the dynamic influences of insect screens and tomatoes crop on air flow movement were modeled by means of the concept of porous medium. Atmospheric radiations contribution was also included in the model. Keywords: Modeling, simulation, greenhouse, microclimate, balance energetic, tomato crop, insect proof, CFD, fluent.

\section{P058. Meat quality traits of Béni Guil lamb raised in eastern Morocco}

Kamal Belhai ${ }^{1,2^{*}}$, F. Mansouri ${ }^{i 1}$, Caid Serghini ${ }^{1}$, M. Sindic $^{2}$, M-L. Fauconnier ${ }^{3}$, M. Boukharta ${ }^{4}$, and Ahmed Elamrani ${ }^{1}$

1 LBPM laboratory, Faculte of Sciences, University Mohamed Ist, University Mohamed Premier, Oujda, Morocco; ${ }^{2}$ QSPA laboratory, Gembloux Agro-Bio Tech, Gembloux, University of Liège, Belgium; 3 Laboratory of General and Organic Chemistry, Gembloux Agro Bio-Tech, University of Liège, Belgium; 4 Haute Ecole Charlemagne, ISla-Huy, Belgium. *Corresponding and Presenting Author: belhaj.kamal90@ gmail.com.

\section{Abstract}

Béni Guil bred is the main ovine breed that dominates livestock farming in the semi-arid region of eastern 
Morocco. No previous data is available on the quality of Béni Guil lamb meat raised on the natural pasture of this area. Therefore, this study aims to provide chemical composition and nutritional traits of this lamb meat. The animals were slaughtered at 33-37 $\mathrm{kg}$ live weight, with a strong fattening condition, corresponding to notation 4, according to the Community Scale (scale 1-5) of Grading Sheep Carcasses EUROP ( 1 ) and $R$ for conformation, according to the SEUROP grid (2). Chemical analysis was conducted on trimmed lyophilised samples of the longissimus lumborum muscle (LLM). Chemical composition traits of LLM, namely dry matter $(25.72 \%)$ protein $(19.43 \%)$ intramuscular fat $(5.14 \%$ and ash percentage $(0.94 \%)$ were similar to those of other indigenous breeds. Total saturated fatty acids (SFA), monounsaturated fatty acids (MUFA) and polyunsaturated fatty acids (PUFA) content in the intramuscular fat was on average of $49.45 \%, 38.48 \%$ and $12.4 \%$ of total fatty acids, respectively. The UFA/SFA and n-6/n-3 PUFA ratios are 1.04 and 3.78 , respectively, and are comparable to those recommended for a balanced diet. Amino acid ( $\mathrm{Aa}$ ) composition analysis conducted by a high-performance liquid chromatography allowed the identification of $17 \mathrm{Aa}$, including eight essential amino acids (His, Met, lle, Leu, Lys, Thr, Val, Phe). The chemical index and the protein digestibility-corrected amino acid score values were 132 and 127, respectively. Keywords: Béni Guil Bred, Lamb meat, Fatty acids, Amino acids, Nutritional quality.

1- La grille EUROP de classement pour l'état d'engraissement [Internet]. Centre Interrégional d'Information et de Recherche en Production Ovine, France. 2011. Available from: www.reconqueteovine.fr.

2- La grille EUROP de classement pour la conformation [Internet]. Centre Interrégional d'Information et de Recherche en Production Ovine, France. 2011. Available from: www.reconquete-ovine.fr.

P059. Effect of silicon on growth and grain yield of wheat

Bendidi Abderrazzak ${ }^{1 *}$, Rachid Dahan', El Housin Bouichou', Chams Dha Khalfi', Mohamed Ibriz², Wafae Sellami ${ }^{3}$, and Mohamed El Yaalaoui ${ }^{3}$

${ }^{1}$ National Agricultural Research Institute, Morocco; ${ }^{2}$
Faculty of Sciences, University Ibn Tofail, Kenita, Morocco; ${ }^{3}$ Faculty of Science and Technology of Fès, Morocco. "Corresponding and Presenting Author: abendidi1@yahoo.fr.

\section{Abstract}

The objective of this study was to determine the effects of silicon on the growth and grain yield of bread wheat and durum wheat. The trial was conducted at the greenhouse at INRA Meknes Morocco using a Randomized Complete Block design with three replications in 2016/2017. Two factors (1- Dose of Silicon; $\mathrm{Si}-=0$ and $\mathrm{Si}+=400 \mathrm{~kg} \mathrm{Si} / \mathrm{ha})$, and 2- Genotype; G1 = bread wheat; Arrehane and G2= durum wheat; Vitron) were tested. Statistical analysis revealed no significant effect of silicon on grain yield and its components for bread wheat and durum wheat. However, we noticed that silicon improved length, volume and root biomass as well as leaf area, plant height and biomass produced for bread wheat, unlike durum wheat. Probably some benefits of silicon (improved resistance to drought, fungal diseases and salinity) were masked because of the conduct the test in the greenhouse. The question that arises is the interest of bringing silicon for wheat in the Saus region of Morocco. Keywords: silicon, bread wheat, durum wheat, growth, yield.

\section{P060. The Chemical and Biochemical Characterization of Moroccan Carob Fruits}

Fatima Ezzahra Eddabbeh ${ }^{1,2^{*}}$, Abdelilah Rahou ${ }^{2}$, and Laila Nassiri ${ }^{1}$

1 Soil \& Environment Microbiology Unit, Faculty of Sciences, Moulay Ismail University, 50050, PB : 298, Marjane, Meknes, Morocco; ${ }^{2}$ Biotechnology and Valvation of Plant Resources, Faculty of Sciences, Moulay Ismail University, P.B. 298, Marjane Meknes, Morocco. "Corresponding and Presenting Author: eddabbeh_ fati_e@yahoo.fr.

\section{Abstract}

The carob tree (Ceratonia siliqua L.) is an agro-sylvopastoral tree specific to the Mediterranean region. In Morocco, it covers an area of about 130.000 hectares. There is a great agro-economic interest in 
the carob treebecause of its low agronomic requirement and its high benefits and Revenues. The results of the chemical and biochemical analyzes made on the Moroccan carob from diverse regions showed a great interest for the industrialists as well as for the valorization of the carob fruit. This explains the induction of new plantations in different regions and the creation of cooperatives for the valorization of its fruits. The present paper synthesizes the chemical and biochemical diversity of carob seed and pulp in Morocco. Keywords: Carob tree, Chemical diversity, Biochemical diversity.

\section{P061. Evaluation of Soil Fertility of Benslimane Region}

Habiba Sehlaoui $\frac{12^{*}}{}$, H. Dakak ${ }^{2}$, A. Zouahri ${ }^{2}$, A. Douaik $^{2}$, A Ghanim ${ }^{i 3}$, and R. Hassikou'

${ }^{1}$ University Mohammed V, Faculty of Sciences, Department of Biology, Mycology and Environment, Rabat, Morocco; ${ }^{2}$ National Research Institute of Agricultural Research, CRRAR, Research Unit on the Environment and Conservation of Natural Resources B.P. 6356 Institutes, Rabat, Morocco; ${ }^{3}$ University Mohammed V, Faculty of Sciences, Department of Chemistry, Rabat, Morocco. "Corresponding and Presenting Author: sehlaovi.habiba@gmail.com.

\section{Abstract}

Morocco has always made the development of the agricultural sector a priority and a strategic choice. But being an essentially arid and semi-arid country, intensive agricultural development has led to an improvement in agricultural production, and it has, on the other hand, generated; degradation of soil quality. This deterioration constitutes a serious danger since it causes a decrease in its fertility. In order to better understand this problem, an assessment of the physico-chemical quality of soils becomes necessary. It is in this context that this study was conducted to examine the chemical fertility of the irrigated perimeter Cherrat, which belongs to the Benslimane region. This region has undergone some economic and particularly agricultural expansion in recent years. A survey of about thirty points was carried out. At first, measurements of $\mathrm{pH}$, electrical conductivity, organic matter content, available phosphorus and exchangeable potassium were made. In a second step, a geographi- cal information system (GIS) was established in order to study the spatial variations of its parameters. Keywords : Soil, fertility, Benslimane, Cherrat.

\section{P062. Agro-morphological analysis of Moroccan sesa- me (Sesamum indicum L.) populations}

Meriem El harfi ${ }^{1,2^{*}}$, Abdelghani Nabloussi ${ }^{2}$, Marouane Jbilou$^{3}$, Hafida Hanine', Hajar Rizki', and Jamal Charafi $^{2}$

1 Laboratory of Bioprocess and Biolnterfaces, Faculty of Science and Technology, Béni Mellal, Morocco; ${ }^{2}$ Research Unit of Plant Breeding and Plant Genetic Resources Conservation, National Institute of Agronomic Research, Regional Agricultural Research Center of Meknès, Morocco; ${ }^{3}$ Regional agricultural Research Center of Afourer, Morocco. "Corresponding and Presenting Author: elharfi.meriem@gmail.com.

\section{Abstract}

Characterization of a given plant material contributes to the optimization of its conservation and its use as a germplasm in breeding programs. The aim of this study was to characterize 33 populations of Moroccan sesame (Sesamum indicum L.) on the basis of agromorphological traits and to estimate genetic diversity among and within populations. The material studied had been collected in 2012 from different zones in the Tadla area and, then, was evaluated in field experiments at the INRA experimental station of Afourer, using randomized block design during 2013 and 2014. The study was done on 13 agromorphological traits, namely growth rate, height of the plant, number of branches, height of the first branch, height of the first pod, number of pods per plant, width of pod, length of pod, number of seeds per pod, thousand seeds weight, seed yield per plant, seed yield per $\mathrm{Ha}$ and oil content. Significant differences between sesame populations in two different environments, Afourer 2013 and Afourer 2014, were observed only for three measured traits: height at the first pod $(75.27$ - $99.46 \mathrm{~cm})$, number of seeds per pod $(55.30$ - 70.35) and thousand seeds weight (3.07 - 3.50 g). However, the environment has a significant effect on the majority of the traits studied. Also, effect of year was more pronounced for Afourer environment, being 2014 more favorable than year 2013. The 
limited genetic diversity among the populations studied might indicate these were very close genetically and likely were derived from a same original variety. Keyswords: Sesame, populations, agromorphological traits, diversity, environment, Morocco.

\section{P063. Evaluation and Selection of Lactic Acid Bacteria as Starter Cultures from Natural Biotopes}

Moussaid Siham ${ }^{1,2^{*}}$, K. Ounine ${ }^{2}$, A. Benali', M. R. Kabour ${ }^{1}$, and E. H. Maadoudi ${ }^{1}$

' Laboratory of Food Technology and quality, Food safety/environment, CRRA, INRA, Avenue Ennasr, BP 415 RP, Rabat, Morocco; ${ }^{2}$ Nutrition, Health and Environment Laboratory, Faculty of Science, University lbn Tofail, B.P. 133 Kenitra. Morocco. "Corresponding and Presenting Author: sihamfstg@gmail.com.

\section{Abstract}

For thousands of years, humans have used microorganisms in the pharmaceutical, food, agronomic and industrial fields. These ubiquitous microorganisms of our environment and our food occupy an increasingly an important place in our life and are currently at the origin of the rise of biotechnology field. Lactic acid bacteria (LAB) are among the microorganisms used in the processing and preservation of food by producing organic acids, proteases, lipases, exopolysaccharides, antibacterial agents, etc. The objective of our study is to isolate and screen LAB that have important technological skills. A total of forty-eight bacteria were isolated from camel milk, goat milk and argan residues. The evaluation of the acidifying ability by $\mathrm{pH}$ monitoring every two hours for twenty-four hours showed that for some bacteria the $\mathrm{pH}$ decreased from 6.7 to 3.8 after 24 hours of fermentation. The OPA method revealed that $14 \%$ of the isolated have a significant proteolytic activity releasing up to $7 \mathrm{mM}$ Glycine. The dosage of fatty acids released by the isolates using the titrimetry method has shown that its amount varies between $0.6 \mu \mathrm{mol} / \mathrm{ml}$ and $2.6 \mu \mathrm{mol} /$ $\mathrm{ml}$. Whereas the anti-free radical activity measured by the DPPH method is $5 \%$ for a sterile skimmed milk while it is maximum for the same milk inoculated by our isolates to reach $34 \%$ compared to vitamin $C$ for certain isolates. $30 \%$ of the isolates have an antibacterial activity against either Staphylococcus aureus,
E. coli or Salmonella enterica or on the three strains at the same time whose diameter of the zone of inhibition of the pathogenic strains is between $2 \mathrm{~mm}$ and $12 \mathrm{~mm}$. On the other hand, $49 \%$ of isolates are able to produce exopolysaccharides responsible for the texture of processed products. Isolated BAL have shown an important property to allow them to be considered as good starter cultures. Keywords: Lactic acid bacteria; acidifying activity; protease; lipase; antioxidant activity.

P064. Genotypic and Environmental Effects on Doublekernelled Fruits of Some Almond [Prunus dulcis (Mill.) D.A. Webb] Cultivars Grown in Northern Morocco

\section{El Hassan Sakar ${ }^{*}$ and Yahia Rharrabti}

Laboratory of Natural Resources and Environment, polydisciplinary faculty of Taza, B.P 1223, TazaGare (Taza), Unviersity Sidi Mohamed Ben Abdellah, Taza, Morocco. 'Corresponding and Presenting Author: hassan.sce@gmail.com / elhassan.sakar@ usmba.ac.ma.

\section{Abstract}

Almond tree [Prunus dulcis (Mill.) D.A. Webb] is an important nut crop commercially grown throughout the world. It is cultivated for its kernel of high commercial and nutritional values. Along with nutritional richness, fruit physical aspect is considered as an important fruit quality trait especially for almond processing and commercialization (consumer acceptance). In this regard, production of double-kernelled fruit is undesirable trait resulting in small and deformed kernels. In this work, we aimed to investigate fruit double kernels from the five widely almond cultivars: Marcona (MAR), Fournat de Brézenaud (FBZ), Ferragnès (FRG), Ferraduel (FRD), and Tuono (TUO). To assess environmental effects on this trait, fruits were sampled from three different environments in northern Morocco: Aknoul (AK), Bni Hadifa (BH), and Tahar Souk (TS) over two harvest seasons 2015 and 2016 . At maturity stage, which fits 89 on the general BBCH phenological scale, samples of $1.5 \mathrm{Kgs}$ were harvested in triplicate. Once at laboratory, for each sample, sub-samples of 100 fruits were used for double-kernelled fruit estimation. Nuts were cracked, and double kernels were counted and expressed in percentage (\%). ANOVA analyses 
outcomes demonstrated significant effects of cultivar, site, harvest season, and their interactions on doublekernelled fruit production. This trait was genetically controlled since genotypic effects were more important and accounted for more than half of the total variance. Moreover, environmental effects were also significant, but climatic factors (harvest season effect) being the most important. Among sites, $\mathrm{BH}$ presented the lowest values of double kernels, while for cultivars FRD and FRG exhibited lower scores of this trait during the two harvest seasons and TUO showed the greatest values. For harvest seasons, 2016 was relatively dry as compared to 2015.2016 presented the lowest values of double kernels. It could be concluded, that double-kernelled fruit trait was a genotypic trait, but climatic factor could enhance or reduce its expression. Keywords: Commercial Almond Cultivars, double kernels, genotypic and environmental effects, market and consumer acceptance.

P065. Development of the tree strawberry varieties (Fortuna, Fragaria and San Andreas)

$\underline{\text { Houda Tahiri }}^{{ }^{*}}$, Ahlam Hamim ${ }^{2}$, and Amal El Amrani ${ }^{3}$

${ }^{1}$ In vitro Culture Laboratory/INRA Tangier, Morocco;

${ }^{2}$ Vegetable biotechnology Laboratory/Faculty of Science Tetouan, Morocco. ${ }^{*}$ Corresponding and Presenting Author: houditahhiri@gmail.com.

\section{Abstract}

The in vitro culture of strawberry plants allows to obtain from a small fragment of a plant placed on a synthetic nutrient medium an infinity of identical healthy plants. In this context, the present work revolves around two main axes. The first axis aims at studying the in vitro micropropagation of strawberry. The response of the explants to various factors, the basic culture medium, growth regulators. In order to have the answer, different concentrations were studied. In vitro germination of strawberry seeds is a mandatory step for the production of the necessary seedlings for micropropagation. The seeds of three cultivars "Fortuna","Fragaria" and "San Andreas" scarified and sterilized by sulfuric acid H2SO2 (36N), (5min); showed a maximum germination capacity after a short passage on the mineral medium of Murashig and Skoog (1962) agar. For the apex mul- tiplication phase, Murashig and Skoog (1962) were selected with hormones with different concentrations of growth regulators ( $1 \mathrm{mg} / \mathrm{I} A \mathrm{AB}, 0,5 \mathrm{mg} / \mathrm{I} B A$, $0,1 \mathrm{mg} /$ ( AG3) for mass production of strawberry plants. For the rooting phase, the results showed that rooting gives better results on the MS medium with different concentrations of $A I B(0.5,1,2$ or $3 \mathrm{mg}$ L-1). 'AIB are different depending on the cultivar and after 5 weeks the seedlings are grown at variable frequencies. The regenerated seedlings are easily acclimatized on a pre-sterilized turmeric-vermiculite substrate. Concerning the second axis, a test of the resistance of the vitroplants to the mites Tetranychus urticae was carried out. The results showed that phytohormone AIB especially at $3 \mathrm{mg} / \mathrm{I}$ can increase the resistance of the vitroplants in the three varieties studied. Keywords:in vitro culture, micropropagation, strawberry, Fortuna, Fragaria, San Andreas, apex, hormones, Tetranychus urticae.

P066. Antibacterial Activity of Cinnamon Essential Oil and its Major Constituent Cinnamaldehyde on Escherichia coli Strains of Avian Origin

Khribch Jamila ${ }^{1^{*}}$, Zrira saadia ${ }^{2}$, Nassik saâdia ${ }^{3}$, EL Houadfi Mohammed ${ }^{3}$, and Oukessou Mohamed ${ }^{1 \S}$

1 Department of Biological and Pharmaceutical Sciences, Hassan II Agronomic and Veterinary Institute, BP 6202, 1010, Rabat, Morocco; ${ }^{2}$ Department of Food and Nutrition Sciences, Agronomic and Veterinary Institute Hassan II, BP 6202, Rabat, Morocco; ${ }^{3}$ Avian Pathology Unit, Department of Pathology and Veterinary Public Health, Agronomic and Veterinary Institute Hassan II, BP 6202, Rabat- Instituts, Rabat, Morocco. "Presenting author: i.khribch.inrh@gmail. com. §Corresponding author: m.oukessou@gmail.com.

\section{Abstract}

The development of bacterial resistance to common antimicrobial agents constitutes a great challenge for actors in the field of human as well as animal health. This situation prompted research of new antimicrobial agents from various sources including mainly plants. The present study consists in evaluating the antibacterial activity of essential oil of cinnamon and its major active constituent the cinnamaldehyde. This activity was evaluated using the usual techniques of antibio- 
gram, aromatogram and determining the minimal inhibitory concentrations (MIC) and the minimal bactericidal concentrations $(M B C)$ in a broth medium against two reference strains (Escherichia coliATCC25922 and Staphylococcus aureus ATCC25923) and strains of E. coli isolated from broiler chickens admitted to the Avian Pathology Unit at IAV Hassan II resistant to the main antibiotics (Enrofloxacin and Florfenicol). In total, cinnamaldehyde has been tested on about twenty bacterial strains, and the essential oil of cinnamon has been evaluated on about 11 Escherichia coli strains. In the second part, the association of cinnamaldehyde with certain antibiotics was tested for possible synergies. Furthermore, cinnamaldehyde kinetic-killing profile was studied using the same germ reference strains. According to the results obtained in this work, essential oil of cinnamon was more active than cinnamaldehyde. The essential oil inhibited the growth of E. coli strains by expressing inhibition halts of 29,3 and $25,1 \mathrm{~mm}$ for the reference strain and strains of avian origin respectively, while cinnamaldehyde gave inhibition zones of 26,5 and 23,23 mm for the reference strains and the wild-type strains respectively. The average values of the MICs obtained with cinnamaldehyde against the reference strains were 0,031\%, 0,041\% for Escherichia coli and Staphylococcus aureus respectively. The same product expressed an average MIC of $0,039 \%$ for strains of avian origin, while the $\mathrm{CMB}$ values were on average $0,031 \%$ for E.coli and $0,026 \%$ for S. aureus. The comparison of MIC and $C M B$ values for bacterial strains indicates that this natural product is bactericidal. Finally, the bactericidal activity of Cinnamaldehyde is close to the time-dependent kind antimicrobial and Cinnamaldehyde-Antibiotics combinations were most likely indifferent agents against both $\mathrm{S}$. aureus and $\mathrm{E}$. coli bacteria. Keywords: Essential oil, cinnamon, cinnamaldehyde, Resistance, Escherichia coli, diffusion method on agar.

\section{P067. Contract Farming Within the Green Morocco Plan: Case of Cereal Sector}

Bouichou El Houssain ${ }^{1,2^{*}}$, A Fadlaoui', K Allali ${ }^{2,3}$, and R Arrach ${ }^{4}$

1 National Institute for Agricultural Research (INRA), Meknes, Morocco; ${ }^{2}$ Agronomic and Veterinary Institute Hassan II (IAV), Rabat, Morocco; ${ }^{3}$ National School of Agriculture (ENA), Meknes, Morocco; 4 Ministry of Agriculture and Fisheries: Directorate of Strategy and Statistics, Rabat, Morocco. "Corresponding and Presenting Author: bouichouelhoussain@yahoo.fr.

\section{Abstract}

Contract farming (CF) has long been practiced but is since the launch of Green Plan of Morocco (PMV) it has regained an important interest with noticeable diversification in structures and involved actors. Under $C F$, aggregators (AG) and aggregated (Ag) make advance agreements and clauses on volume, quality, time of delivery, use of inputs, and price or pricing formula. The main purpose of the study is to characterize and analyze principles governing the contracts of cereal aggregation project, implemented on a national scale. Emphasizing the objectives, details the undertakings of the contracting parties, as well as, State's undertakings. These investigations sensed the implementation of 120 Agricultural Aggregation Project (AAP) in the main cereal crops. The number of the AAP for cereals having received the certificate of aggregation amounts to 7 . The project aggregation contract of cereals crops analysis launched at national level, allowed revealing that they are structured in five clauses categories: 1) inputs supply, technical support and productions methods; 2) quantities, quality and terms of payment; 3 ) elements of the fixing of purchase prices and trading conditions; 4) management and sharing of risks; and 5) mediation, agree dispute resolution. This preliminary analysis stressed, inter alia, that great efforts were made of the regulation, and formalization of the contractual relationship. However, the contracts are faced with many shortcomings and insufficiency. Indeed, the principal ones concern the heterogeneous contents which do not include quality advantages and the lack of the Ag's implication in the negotiation process. The latters are the ones who support all the risks inherent to the production. Knowing that he use of specialized bodies for the drafting of contracts is rarely carried out. Significant efforts remain of their content and the standardization of their structure which would take into account the guidelines of the law on aggregation. As well as other specific interventions, based on information, training and dissemination of the expected benefits from coordination should increase farmers' willingness to join contract farming. Keywords: 
Agricultural Aggregation Project, Cereal, Contractual clauses, Contract farming, supply chains, Vertical Coordination.

P068. Effect of feeding argan by-products on the quality of fattening lamb's meat

Sanaa Moutik $\frac{1,2^{*}}{}$, N. Lakram ${ }^{2,3}$, M. Bendaou ${ }^{2}$, E. H. El Maadoudi $^{2}$, R Kabbour ${ }^{2}$, N. Essafi', and N El Housni ${ }^{2}$

1 Physico-chemistry laboratory for inorganic and organic materials, Ecole Normale Supérieure, Mohammed V University, Mohamed Bel Hassan El Ouazzani Avenue, RP BP 51 18, Rabat, Morocco; ${ }^{2}$ INRA, RCARRabat, P.O. Box 6570, Rabat Institutes, Rabat Morocco ; ${ }^{3}$ Laboratory of zoology and general biology, Faculty of Sciences, Mohammed V University, Ibn Battuta 4 Avenue RP BP 1014, Rabat, Morocco. ${ }^{*}$ Corresponding and Presenting Author: moutik.sanaa@ gmail.com.

\section{Abstract}

Conventional method of fattening of lambs consists of using rations based on concentrate, to ensure a faster growth. Indeed, the studies accomplished have shown that the daily food intake of lambs is composed of about $80 \%$ of cereals with $50 \%$ of manufactured concentrate, $50 \%$ between barley and corn. However, the increase of grain's price, claim to review ways of lamb's feeding. An interesting approach is to use the by-products of agriculture. Compared to other byproducts, the pulp and the meal of the Argan offers nutritional advantages, they are rich respectively in sugar $(12 \%)$ and protein $(42 \%)$. The purpose of this work is to test the effect of incorporating these byproducts into the lamb's diet. The feeding trials were carried out in real medium on two lots of lambs of the Sardi breed. One fed with Argan tree by-products and the other fed a traditional diet. The results obtained so far are very encouraging.Keywords: Meal, pulp, Argan, lambs, fattening.

P069. Characterization of Flavonoid by HPLC-MS analysis, Antioxidant and Antibacterial potential of Capparis spinosa $L$.

Imane Tagnaout ${ }^{1,2^{*}}$, Hannou Zerkani ${ }^{1,2}$, Zakariya Khi$\mathrm{ya}^{1}$, Aziz Drioiche ${ }^{1,2}$, and Touriya Zair ${ }^{1,2}$
1 Research team of Chemistry Bioactive Molecules and the Environment, University Moulay Ismail, Faculty of Sciences, BP 11201 , Zitoune, Meknès, Morocco; ${ }^{2}$ Laboratory of Materials Chemistry and Biotechnology of Natural Products, University Moulay Ismail, Faculty of Sciences, Zitoune, Meknès, Morocco. ${ }^{\text {*Cor- }}$ responding and Presenting Author: i.tagnaout@edu. umi.ac.ma.

\section{Abstract}

Capparis spinosa L. is a species which has a great importance in the field of traditional medicine in arid and semi-arid regions whose nutritional and medicinal value remains ambiguous in Morocco. It is in this context that our work is aiming at the recovery of this species through a phytochemical study and an evaluation of antibacterial and antioxidant activities of flower buds of Capparis spinosa L. spontaneous collected and sold by Nour cooperative within the region of Zerhoun, Morocco. We achieved the solid-liquid extraction by two different methods in order to optimize the extraction yields of polyphenols; maceration and soxhlet by using two mixtures of solvents: methanol / water and acetone / water at $70 \%$. The best yields are obtained with methanol / water mixture; $46 \%$ for soxhlet against $31 \%$ for maceration. The quantitative analysis of phenolic is being performed by colorimetry, extracts showed that the soxhlet method is more effective than the maceration. The qualitative analysis performed by HPLC / UV-ESI-MS also reported the existence of the same flavonoid glycosides in both hydromethanolic extracts in which the most important are the derivatives of kaempferol, quercetin and isorhamnetin. The antioxidant activity is determined by the DPPH test, showed that the aqueous-methanolic extracts obtained by soxhlet and maceration expressed some approximate antioxidant powers with IC50 of respectively $0.73 \pm 0.01$ and $0.84 \pm 0.01 \mathrm{mg} / \mathrm{ml}$. However, the antibacterial activity evaluated against pathogenic strains such as Staphylococcus aureus, Escherichia coli, Klebsilla pneumoniae, revealed the ineffectiveness of various extracts against the most tested strains. Keywords: Capparis spinosa L., Zerhoune, polyphenols, flavonoids, HPLC, antibacterial, antioxidant activity. 
P070. Molecular and biochemical profiling of Thymus satureioides genotypes cultured under in vitro and in vitro environments

\section{Aicha Nordine ${ }^{*}$-and Abdelmalek El Meskaovi}

Phytobiotechnology Unit, National Agency of Medicinal and Aromatic Plants, PB 159, Taounate, Morocco. *Corresponding and Presenting Author: aicha.nordine@usmba.ac.ma.

\section{Abstract}

In this study, the in vitro and in vivo essential oil (EO) composition and genetic variability of six micropropagated genotypes of Thymus satureioides Coss., a Mediterranean medicinal and aromatic plant, were analyzed by gas chromatograph coupled to a mass selective detector (GC/MS) and randomly amplified polymorphic DNA (RAPD). Yield and composition of the essential oil varied between genotypes. Cluster analysis based on RAPD data and EO grouped the six genotypes in three groups in both culture conditions, thus showing considerable intraspecific genetic and chemical variations. Applying the Mantel test, the result showed a significant correlation between the two proximity matrices; RAPD and EO obtained from in vitro genotypes whereas this correlation was not observed when using the EO obtained from the in vivo genotypes. Keywords: Carvacrol, Essential oil, In vitro environment, Thymol, Thymus satureioides.

\section{P071. Response of two varieties of banana (Musa acu-} minata) small dwarf and large dwarf in vitro to salt stress

Meriem Belfakih ${ }^{1^{*}}$, Mohammed Ibriz ${ }^{1}$, and Abdelmjid Zouhair $^{2}$

Laboratory of Genetics Biometry, Faculty of Sciences, University Ibn Tofail, BP 133. 14 000. Kenitra, Morocco. ${ }^{*}$ Corresponding and Presenting Author: $\mathrm{m} \_$belfakih2000@yahoo.fr.

\section{Abstract}

The objective of this study is to evaluate the effect of salt stress $(\mathrm{NaCl})$ on the in vitro regeneration of banana and the possibility of using this technique to improve the tolerance of this species to salinity. Previously, the rejects were disinfected with $8 \%$ bleach and then grown in Murashige and Skoog medium (1962) containing growth hormones (Appendix 1). The medium was fed with different salt concentrations $(0,2,4$ and $6 \mathrm{~g} / \mathrm{I}$ of $\mathrm{NaCl})$. The test was conducted at a temperature of 28 to $30^{\circ} \mathrm{C}$ with artificial illumination and a photoperiod of $16 \mathrm{~h}$. The results obtained show that both varieties have regeneration of shoots despite saline treatment. However, salt affects leaf length of the explants and the regeneration rate. So, the increase in saline concentration induce to a decrease in the number of shootings per bud. The small dwarf variety appears to be sensitive to the salinityeffect of the medium at all NaClconcentrations at the regeneration stage and the seedling stage. Keywords: Salt stress, regeneration, growth, in vitro, banana.

\section{P072. Impact of the Combination of Salt Stress and Mineral Nutrition on the Growth and Biomass of Alfalfa (Medicago sativa $L$ ).}

Meriem Belfakih ${ }^{1^{*}}$ and Mohammed Ibriz ${ }^{1}$, and Abdelmajid Zouhair ${ }^{2}$

Laboratory of Genetics Biometry, Faculty of Sciences, University Ibn Tofail, BP 133. 14 000. Kenitra, Morocco. ${ }^{*}$ Corresponding and Presenting Author: $m \_b e l-$ fakih2000@yahoo.fr.

\section{Abstract}

This work aims to study the combined salt stress effects $(0.6$ and $12 \mathrm{~g} / \mathrm{I} \mathrm{NaCl})$ and the conditions of normal $(\mathrm{HN})$ and limiting (HD) mineral nutrition, on growth and mineral nutrition of three varieties of alfalfa (Sirever, Demnate 203, and Riche). The study was done under controlled conditions in a growth chamber for one month. The results obtained in this test showed that the salt treatment leads to a net reduction of the aboveground and root biomass upon addition of $6 \mathrm{~g} / \mathrm{l}$. This detrimental effect of salinity becomes less under normal supply conditions, with the exception of the 'Demnate 203' variety which showed the highest yield under limiting supply conditions. Also, saline treatment leads to an accumulation of $\mathrm{Na}+$ cation and a reduction in $\mathrm{K}+$ absorption. The 'Siriver' variety was more sodium-inclusive under normal mineral sup- 
ply (HN) conditions. In addition, the 'Demnate 203' ecotype produced more dry matter under salt conditions and limiting mineral supply (HD). Keywords: Alfalfa, Medicago sativa, salinity, mineral nutrition.

P073. Survey and Multivariate Characterization of Some Figs Genotypes (Ficus Carica L.) Prospected in Northern Morocco

Lahcen Hssaini ${ }^{1,2 *}$, Hafida Haninie ${ }^{2}$, Rachid Razouk', Ibtissame Guirrou ${ }^{1,3}$, Assia Ejiilani ${ }^{1,2}$, Abderrahman Mekaoui ${ }^{1}$, and Jamal Charafi ${ }^{1 \S}$

${ }^{1}$ National Institut of Agricultural Research (INRA), regional center of Meknes, Morocco; ${ }^{2}$ Laboratory of Bioprocess and Bio-Interfaces, Faculty of Science and Technology, Beni Mellal, Morocco ; ${ }^{3}$ Laboratory of food technologies, Qualipole, Meknes, Morocco. "Presenting Author: hssaiini@gmail.com. ${ }^{\S}$ Corresponding author: jcharafi@gmail.com.

\section{Abstract}

In order to collect some of the most known and cultivated fig genotypes in the northern regions of Morocco, the survey of family orchards in the area was performed. Tree main fig growing regions were included into the field inventory (Taounate, Ouazzane and Meknes). All collected material was morphologically and pomologically characterized according to internationally accepted descriptors for Ficus carica L. The colorimetric analysis was carried out according to CIE. All data were standardized $(\mu=0$ and $\sigma$ $=1$ ) to have a comparable scale. Hence, analysis of variance has shown a very high significant level of variability $(p<0.05)$ among evaluated genotypes for all variables. The principal component analysis (PCA) has revealed two mean groups with a total inertia of $78.7 \%$ based on the quantitative traits. Otherwise, the genotypes "Ghani" and "Ounq Hmam_PS14" have been largely distinguished from the two groups. The colorimetric principal component analysis, has shown tree distinctive and homogeneous groups with a total inertia of $90.92 \%$. Thus, the first group contains the brightest and clearest fruit skin color (very high values of $L^{*}$ and $C^{*}$ coordinates), the second is related to fruits with skin color less bright and tending to be purple. While the last group characterize genotypes with a dark skin color (low values of $L^{*}$ and negative ones for $a^{*}$ and/or $b^{*}$ coordinates). Qualitative characterization has shown a dominance of globose $(74 \%)$ and rounded $(61 \%)$ shape of figs. Most of genotypes have skin ribes (82\%) and $78 \%$ did not present the drop at the ostiole. Hierarchical ascendant classification (HAC) was been performed using the Euclidian distance based on all 38 variables (color, morphological and pomological descriptors) to decide the ultimate number of clusters by which the accessions could be assessed. The result has shown two main clusters, each one is subdivided distinctively into two homogeneous subgroups with a main distance of 10. Every subgroup is discriminated following a specific group of characters. With the exception of "Ghoudan" and "Ghani", all genotypes with the same denominations were clustered into the same group. Keywords: Northen Morocco, Ficus Carica, pomology, morphology, colorimetric.

\section{P074. Assessment of Genetic Diversity in Two Moroc- can Populations of Walnut (Juglans regia L.) Using ISSR Markers}

Jamal Charafi ${ }^{*}$, Abdellah Kaiii, and Rachid Razouk

National Institute of Agricultural Research (INRA), Regional Center of Meknes, Morocco. * Corresponding and Presenting Author: jcharafi@gmail.com.

\section{Abstract}

Walnut (Juglans regia) covers an area of 7600 ha in Morocco and performs an important socio-economic role. Indeed, it constitutes a significant source of income for farmers despite the many constraints related to its production and valorization. The fact that seeds are the only mean of propagation used by farmers has largely contributed to its diversification. Prospection was made in two main cultivation regions: Haouz in the High Atlas and Midelt in the Middle Atlas. Thus, assessing the genetic diversity of the walnut's genetic resources is a first step towards conservation and the elaboration of a breeding program. A characterization of 33 Moroccan walnut genotypes from these regions - 25 genotypes from the Haouz region and eight genotypes from the Midelt region - was carried out. In addition to eight Bulgarian varieties by 13 ISSR, primers can detect a large genetic diversity. A total of 120 markers were detected and the number 
per primer varied between 7 and 13 with an average of 9 bands. This number reflects the high level of polymorphism within the studied genotypes. The primers generated $75 \%$ of polymorphic fragments and the pairwise comparison of the 41 genotypes shows that all genotypes pairs are distinct by 10 to 50 markers. These large differences are due mainly to the multiplication mode dominated by seeding which favors the diversity explosion. This high diversity between genotypes is confirmed by the genetic distances obtained and hierarchical analysis using the UPGMA method. Results show a minimum distance of $58 \%$, a maximum of $91 \%$ and an average of $76 \%$; the geographic structure is evident. The genotypes subgroups of the Midelt region are distinct from those of the High Atlas and the subdivisions into subgroups by village are remarkable. The presence of some genotypes in subgroups of other areas is explained by exchanges of plant material (nuts) between the agricultures. Bulgarian varieties are distinct from local genetic material which confirms the specificity of the local walnut's genetic patrimony. Keywords: Walnut, Juglans regia., Genetic diversity, Molecular markers, ISSR.

P075. Earthworm feeding activity and mucus secretion can decrease entomopathogenic nematodes activity as biological control agents

Maryam Chelkha ${ }^{1,2^{*}}$, Rubén Blanco-Pérez ${ }^{2,3}$, Francisco Ángel Bueno-Pallero ${ }^{2}$, Abdellatif El Harti ${ }^{1}$, Souad Amghar $^{1}$, and Raquel Campos-Herrera ${ }^{2 \S}$

1 Research Team "Lombricidae, Improving Soil Productivity and Environment" (LAPSE), Ecole Normale Supérieure (E.N.S.), Centre Eau, Ressources Naturelles, Environnement et Développement Durable (CERN2D), Mohammed V University in Rabat, Avenue Mohamed Bel Hassan El Ouazzani, BP : 5118. Takaddoum - Rabat Morocco; ${ }^{2}$ MeditBio, Centre for Mediterranean Bioresources and Food, Universidade do Algarve, Campus de Gambelas, FCT Ed. 8, 8005 139 Faro, Portugal; ${ }^{3}$ Department of Agriculture and Food, Universidad de La Rioja, Calle Madre de Dios, 51, 26006, Logroño, Spain. * Corresponding Author: marychalkha@hotmail.com. ${ }^{\S}$ Corresponding Author: rcherrera@ualg.pt.

\section{Abstract}

Earthworms are well-known beneficial organisms associated to plant production by indirect action (i.e. changes in soil aeration, structure and fertility) and by direct action (i.e. enhancing decomposition of organic matter). In addition, earthworms might promote the dissemination of beneficial soil organisms, including biological control agents, such as entomopathogenic nematodes (EPNs). Despite the fact that EPNs result innocuous against earthworms, and their displacement can be favoured by the earthworm activity, the impact of other earthworm activities such as feeding behaviour or mucus production is poorly understood. We hypothesized that feeding activity might decrease the viable EPNs in the soil, reducing their ability to kill insects, whereas the presence of mucus will not affect the biocontrol skills. We evaluated the feeding activity and mucus secretion of the earthworm Eisenia fetida (Haplotaxida: Lumbricidae) in combination with three EPN species: Steinernema feltiae, S. glaseri and S. carpocapsae (Rhabditida: Steinernematidae). First, we tested whether the presence of mucus can alter the infectivity of the three EPNs by using Galleria mellonella (Lepidoptera: Pyralidae) as model insect. Treatments included controls (water and mucus) and two concentrations ( 1.5 and $10 \mathrm{JJs} / \mathrm{cm} 2$ ) per EPN species in the presence and absence of mucus. Second, we evaluated the killing ability of EPNs against $G$. melllonella larvae when applied in autoclaved soil alone or in combination with earthworms or mucus. Our results showed that the presence of mucus significantly reduced the larval mortality caused by $\mathrm{S}$. feltiae and S. glaseri, while S. carpocapsae was not affected. Similarly, the presence of earthworm and mucus reduced the larval mortality caused by S. feltiae and $\mathrm{S}$. glaseri, resulting the mucus the most restrictive treatment. Our results illustrate how the presence of earthworms or their secretions (mucus) can alter the long-term persistence in the soil of certain EPN species as well as its action as biological control agents in a dense-dependent and species-specific manner. Further studies will investigate whether this trend is general to other EPN species as well as other entomopathogenic agents to establish to which extend the combined application of these beneficial organisms is compatible. Keywords: earthworms, entomopathogenic nematodes, biological control, multitrophic interactions 
P076. Analysis of the oil content and fatty acid composition of 94 cultivars of olive

Tfarah Boutzankad 1*, A. Mhais', S. Mousavi ${ }^{2}$, C. El Modafar ${ }^{1}$, A. El Antari ${ }^{3}$, A. Moukhli ${ }^{3}, H$. Zaher $^{3}$, and M. A. Tadlaoui'

1 UCAM, FSTG, Bd. A. Khattabi, Marrakech, Morocco ; 2 CNR- ISAFoM 128, Via della Madonna Alta 06128 Perugia, Italy ; 3 INRA, CRRA, Marrakech, B.P.533 Menara, Marrakech, Morocco. "Corresponding and Presenting Author: tfarah.boutzankad@ gmail.com.

\section{Abstract}

Olive oil contains a high percentage of unsaturated fatty acids, that is why it is considered to have a big nutitional interest. In litterature, the selection of olive varieties is taken on the basis of their argonomic characters. In the present work, our objective is to characterize the nutritional quality of 94 cultivars (Core Collection) in term of the oil content and the fatty acids composition in the fruits and the correlation with the genetic profile of core collection cultivars, divided into their genetic structre groups (East, Center and West. The statistical analysis showed significant effect of cultivar and interaction cultivar $x$ replicates for the traits; oil content, UnSATFA/SFA ratio, palmitic, oleic and linoleic acids percentage. Then there is only genetic effect for the palmitic and stearic acids percentage. Indeed, regarding to the admixed cultivars from core collection, the oil content is high in Eastern group followed by Western and Centeral groups. Whereas, the unSATFA/SFA ratio is high in Western group followed by Eastern and then Central. Therefore, the cultivars which have high linoleic acid content are from East, followed by the Centeral and the Western groups. The Principal Components Analysis (PCA) carried out on data summarizes $69,30 \%$ of the general information on the first two dimensions. As a result, three groups were distinguished : the first is characterized by high values of oleic acid percentage and unSATFA/SFA ratio as well as low palmitic acid percentage. The second contains individuals with intermediate values and last group gave high palmitic acid, low oleic acid and unSATFA/SFA ratio. This investigation will be in process for two more years in order to estimate the year effect and to quanti- fy the genetic effect via the heritability estimation. Keywords: Oil content, Fatty acids composition, Core Collection, Olive oil.

P077. Livestock Farming System and Reproductive Performance of Local Goat in the Argan Tree Park (Arganeraie) of the Agadir Region in Morocco

\section{Houda El Kheyyat ${ }^{*}$ and Saïd El Madidi§}

Biometrics and Bio Resources, Laboratory Biotechnologies and Natural Resources Development (LBVRN), Faculty of Sciences, University Ibn Zohr, Agadir, Morocco. Presenting Author: elkheyyathouda@yahoo.fr. §Corresponding Author: s.elmadidi@uiz.ac.ma.

\section{Abstract}

A study was conducted using a structured survey among 35 goat farmers randomly selected and distributed in 9 villages in the Arganeraie of the Agadir region. The livestock system is of an traditional extensive type whose diet is based on sylvo-pastoral resources. Herd management is permanent wandering with confinement during rainy days. Only $37 \%$ of the respondent goats' farmers can provide their goats with drinking water, only $5.71 \%$ who have drinking troughs. The living area per goat is less than $\left(1.5 \mathrm{~m}^{2}\right.$ / goat) for $70 \%$ of goats' farms and less than (1 $\mathrm{m}^{2} /$ goat) for $57 \%$ of the goats' farms surveyed. The farmers surveyed were mostly illiterate $(74.29 \%)$ with an age between 27 and 77 years old. The average herd size is 82 animals per farmer and $80 \%$ of the farms have between 10 and 100 animals. Animal vaccination is only provided for $25.71 \%$ of goat farms and $74.29 \%$ of them do not have access to health care. All goat herds are composed of $72.54 \%$ females and $27.46 \%$ males. Out of a total of 3190 goats observed, 459 are male goats, 1493 female goats, 417 male kids and 821 female kids. The goat herd is heterogeneous and composed mainly (86.4\%) of 3 local breeds (Alas, Barcha and Ghazalia) which represent respectively $57.4 \%, 22.3 \%$ and $6.7 \%$. The number of kiddings is 1 for $71.4 \%$ of farmers, 1.5 for $28.6 \%$ of them. $8.6 \%$ of farmers do not know the age at first birth and $82.8 \%$ of them, this age is less than or equal to 12 months. The prolificacy rate is $114.3 \%$ and the abortion rate is $28.4 \%$. The mortality rate for adults was $17.92 \%$ and that for the kids 
was $12.76 \%$. The main barriers to goat production in the region are food, housing, health and lack of subsidies. Keywords: Livestock farming system, Argan tree park (arganeraie), reproductive performance.

\section{P078. Preliminary standards for apple fertilization in the middle Atlas Mountains of Morocco}

Rachid Razouk ${ }^{*}$, Abdellah Kajii, Jamal Charafi, Mohamed Alghoum, and El Houssain Bouichou

Regional Agricultural Research Center of Meknes, B.P 578, Meknes, Morocco. 'Corresponding and Presenting Author: razouk01@yahoo.fr.

\section{Abstract}

Until now, interpretation of soil and leaf analyses for the Moroccan apple orchards is based on reference norms established on other countries such USA, France, Netherlands, United Kingdom and South Africa. Establishment of norms under local conditions is therefore necessary for an efficient management of fertilization in Moroccan orchards taking into account local practices and production performances. For this objective, a work was carried out to evaluate use efficiency of nitrogen, phosphorus, potassium, calcium and magnesium in adult apple orchards in the middle Atlas of Morocco (Imouzzer Kandar) and to establish preliminary reference norms for fertilizing this crop under local conditions. The study was based on soil and leaf analysis and data regarding farming practices and yield level on forty apple orchards (cv. Golden delecious/MM106) where nineteen are growing on silty-clay soil and twenty-one on sandyloam soil. The results showed significant correlations between leaf content for each nutrient and yield level following polynomial equations, thereby indicating local reference norms for apple leaf analysis: 2.80$3.10 \%$ for nitrogen, $0.20-0.22 \%$ for phosphorus, $1.90-2.10 \%$ for potassium, $1.30-1.45 \%$ for calcium and $0.25-0.28 \%$ for magnesium. The correlations between leaf and soil contents were significant, thereby indicating apple needs in nitrogen fertilizer: $88 \mathrm{~kg} /$ ha on silty-clay soil and $130 \mathrm{~kg} / \mathrm{ha}$ on sandy-loam soil. Moreover, were determined references norms for soil richness in phosphorus (92-126 ppm on silty-clay soil and 82-106 on sandy-loam soil), potassium (350415 ppm on silty-clay soil and $320-410$ ppm on san- dy-loam soil), calcium and magnesium for an apple yield objective of $26 \mathrm{t} / \mathrm{ha}$ corresponding to the average yield obtained in the study area. The found norms for leaf analysis are less than international standards because of feebleness of yield level in the study region, largely related to deficient cultural practices adopted by farmers. In addition, investigation of leaf nutrients ratios $\mathrm{N} / \mathrm{K}, \mathrm{N} / \mathrm{Ca}, \mathrm{K} / \mathrm{Ca}, \mathrm{K} / \mathrm{Mg}$ and $\mathrm{Ca} /$ $\mathrm{Mg}$ showed that there was a disharmony in uptake of these nutrients originating particularly from high soil richness in $\mathrm{Ca}$ and $\mathrm{Mg}$. Taking into account these considerations, the found references norms can be applied only under the adopted farming practices. Nevertheless, by improving local practices, reference values may change. Keywords: Morocco, apple tree, soil analysis, leaf analysis, fertilization standards.

\section{P079. Effect of potassium source and rate on produc- tivity and quality of strawberry fruit}

\section{Amal Nakro* ${ }^{*}$ and Ahmed Bamouh ${ }^{\S}$}

Department of Plant Production, Protection and Biotechnology, Hassan II Institute of Agronomy and Veterinary Sciences, BP 6446 Madinate Al Irfane, Rabat, Morocco. "Presenting author: a.nakro@iav.ac.ma. §Corresponding author: a.bamouh@iav.ac.ma.

\section{Absract}

The purpose of this research is to investigate the effect of potassium fertilization source and rate on productivity and quality parameters of strawberry. The experiment was conducted in 2016-2017 at the experimental greenhouse of Hassan II Institute of Agronomy and Veterinary Sciences in Rabat. Strawberry plants of the cultivar 'San Andreas' were planted on November 6th in 12-liter pots equipped with a gravity fertigation system. Tested treatments were two potassium sources: (1) Sulphate of potash for all the growing cycle (SOP/SOP) (2) Nitrate of potash during the vegetative period and Sulphate of potash during the fruit production period (NOP/SOP) combined with four potassium rates of $100,200,300$ and $400 \mathrm{~kg} / \mathrm{ha}$ of $\mathrm{K} 2 \mathrm{O}$. The experimental design is a split-plot of eight treatments with three replications. The potassium sulfate source was superior to potassium nitrate on the improvement of productivity and quality parameters of strawberry fruit. The potassium 
sulphate improved the number of flowers by $16 \%$, the fruit production by $21 \%$, the titrable acidity content by $0.5 \mathrm{~g} / \mathrm{I}$ and the sugar content by $3 \%$. The highest yield was recorded in plants treated with $300 \mathrm{~kg} /$ ha of $\mathrm{K} 2 \mathrm{O}$ of potassium sulphate. The rate of 300 $\mathrm{kg} / \mathrm{ha}$ of $\mathrm{K} 2 \mathrm{O}$ gave also superior results regarding quality parameters of strawberry fruit. This optimum potassium rate enhanced the number of flowers by $35 \%$, the fruits produced by $44 \%$, the sugar content and the titrable acidity content by $0.9 \mathrm{~g} / \mathrm{l}$. Keywords: Strawberry, fertilization, potassium, source, rate, potassium sulphate, potassium nitrate, productivity, quality, sugar content, titrabale acidity.

P080. Intensive urbanization at the expense of farmland

EL Hirch Kaoutar ${ }^{1,2,3^{*}}$, Ait Yacine Z. ${ }^{2}$, Idrissi N. ${ }^{3}$, and Wahid N. ${ }^{1 \S}$

1 Équipe d'Écologie et Développement Durable (EEDD), Département de Sciences de la Vie, Faculté des Sciences et Techniques, Université Sultan Moulay Slimane, Béni-Mellal, Morocco; ${ }^{2}$ Équipe de Génie de l'Environnement, Département de Sciences de la Vie, Faculté des Sciences et Techniques, Université Sultan Moulay Slimane, Béni-Mellal, Morocco; ${ }^{3}$ Équipe de Traitement de l'Information et Aide à la Décision, Département de l'Informatique, Faculté des Sciences et Techniques, Université Sultan Moulay Slimane, Béni-Mellal, Morocco. "Presenting author: kaoutarelhirch.cha2@gmail.com. ${ }^{\S}$ Correspondig author: wahid2na@hotmail.com.

\section{Abstract}

Currently, the modern city has lost these properties and these landscape reflexes related to the phenomenon of urbanization. In fact, urban sprawl alters land use and the appearance of the countryside, which contributes to the accelerated evolution of habitats with regard to areas devoted to green spaces. Despite the laws for the preservation of agricultural land, urban sprawl is intensifying to the detriment of land with high agronomic potential, increasingly in the face of economic growth. This is worsening more and more in the absence of a sustainable urbanization strategy. Several studies have confirmed that unsustainable urban intensification has environmental consequences; risks on agricultural production, the disruption of agricultural ecosystems, the landscape quality of the city, and the quality of the climate. The current challenge is to define indices of the degree of regression of agricultural land or agricultural biodiversity, and the cost of loss generated by urban intensification. In this context, our work is to trace the evolution of urbanization in the city of Beni-Mellal, in order to evaluate its impact on the structuring of agricultural biodiversity and on the phenomenon of global warming. The evaluation and anticipation of its impacts are of interest to both scientists and territorial managers. Keywords: Urban intensification, agricultural biodiversity, global warming, Béni Mellal.

P081. Ability of non-pathogenic Fusarium oxysporum strain Fo47 to suppress Rhizomania disease of sugar beets in Morocco

Fatima Novayti ${ }^{1,2^{*}}$, Ilham Madani ${ }^{2}$, Abdessalem Tahiri ${ }^{1}$, Abdelali Blenzar ${ }^{2}$, and Rachid Lahlali ${ }^{1}$

1 Phytopathology Unit, Department of Plant Protection, Ecole Nationale d'Agriculture de Meknès (ENAMeknès), Km 10, Route Haj Kaddour, BP S/40, Meknès 50001, Morocco; ${ }^{2}$ Moulay Ismail University, Faculty of Sciences. Department of Biology PO Box 11201 Zitoune Meknes, Morocco. "Corresponding and Presenting Author: fatimanovayti@gmail.com.

\section{Abstract}

Rhizomania is one of the most devastating diseases of sugar beet worldwide. The disease poses a serious threat to Moroccan production and it is capable of significantly decreasing quality and yield of sugar beet plantations. The long-term survival of its fungal vector (Polymyxa betae) in soil makes it a very difficult disease to manage. Therefore, this study investigated the potential of a non-pathogenic fungal Fusarium oxysporum strain Fo47 to control Polymyxa betae. This biocontrol agent was applied as soil treatment, seed treatment, or a combination of the both treatments. A bio-test was performed on treated soil. After four weeks of culture, the roots of sugar beet seedlings were retrieved and analyzed by the DAS-ELISA test. Results indicated that F. oxysporium Fo47 reduced the activity and survival of $P$. betae when compared to a reference biocontrol agent Trichoderma harzianum, 
which only revealed significant in reducing the viral load of Beet Necrotic Yellow Vein Virus (BNYVV) as seed treatment. The non-pathogenic Fusarium oxysporum Fo47 was more effective as soil treatment and allowed almost the same reduction of BNYVV virus concentration as T. harzianum 908. Therefore, our findings emphasizes that the performance of the biocontrol agent depends on the method of application. Keywords: Beet Necrotic yellow vein Virus; Biocontrol, Fusarium oxysporum strain Fo47; Trichoderma harzianum strain 908.

P082. Preliminary study of the effect of humic substances on saffron growth and corms production

Abdelghani Tahiri ${ }^{*}$, Youssef Karra, Meriyem Koufan, Naima Ait Aabd, and Abdelaziz Mimouni

National Institute of Agronomic Research, CRRA, Agadir, Av. des FAR, BP. 124, Inezgane, Morocco. ${ }^{*}$ Corresponding and Presenting Author: aghanitahiri@ yahoo.fr.

\section{Abstract}

Among the 85-species belonging to the genus Crocus, saffron (Crocus sativus L.) is a monocotyledonous plant that belongs to the Iridaceae family. It has been cultivated for its stigmas that provide the most expensive spice which has been valuable since ancient times for its odoriferous, coloring, and medicinal properties. Saffron, is an autumn-flowering, triploid male -sterile plant propagated vegetatively by means of corms as the flowers are sterile and fail to produce viable seeds. Low multiplication rates and fungal infestation of corms reduce the productivity and quality, thereby restraining the availability of planting material. To overcome these problems, in this study an experiment was conducted to evaluate the potential effect of commercial humic substances (as biostimulant) on saffron growth, quality and corms production. Keywords: Saffron, Corm production, Crocus sativus, Humic substances.

P083. Study of the antioxidant compounds of Artemisia mesatlantica maire

Sanae Amine $^{1,2^{*}}$, Fatima Zahra Radi ${ }^{1,2}$, Zakaria Khi$\mathrm{ya}^{1,2}$, Smail Amalich ${ }^{1,2}$, Malika Mahioubi ${ }^{1,2}$, Mohamed
Bourakhouadar ${ }^{1,2}$, and Touriya Zair ${ }^{1,2}$

1 Research team of Chemistry Bioactive Molecules and the Environment, University Moulay Ismail, Faculty of Sciences, BP 11201, Zitoune, Meknes, Morocco; ${ }^{2}$ Laboratory of Materials Chemistry and Biotechnology of Natural products, University Moulay Ismail Faculty of Sciences, B.P. 11201 Zitoune, Meknès, Morocco. ${ }^{*}$ Corresponding and Presenting Author: aminasanae@hotmail.fr.

\section{Abstract}

Artemisia Mesatlantica maire is an endemic species of Morocco, belonging to the Asteraceae family. Located in the Moroccan Atlas region, it is very requested by the local population for its therapeutic properties. However, because of the limited studies conducted on this species, and in order to contribute inits valorization, we selected it in full bloom in the Middle Atlasregion for a phytochemical study and an evaluation of its antioxidant properties. The phytochemical screening of $A$. Mesatlantica maire revealed its richness in polyphenols, sterols, triterpenes and mucilage. The extraction yield of the total polyphenols by macerationin a methanol-water mixture $(80 / 20)$ was $10.10 \%$. The determination of the total phenols by the Folin-Ciocalteu method, after fractionation of the polyphenolic extract ofA. Mesatlantica maire, showed the abundance of these compounds in the butanol fraction. For the total flavonoids essay, using Aluminum Trichloride, the Ethyl Acetate and Butanol fractions of $A$. Mesatlantica maire were the richest. In addition, the raw extract was particularly rich in condensed tannins. The valuation of the antioxidant activity by reduction of iron (FRAP) and trapping of the free radical DPPH* showed that all the extracts have a moderate antioxidant power. These first studies on A. mesatlantica maire of the Middle Atlas revealedits abundance in important chemical compounds, and due to the ability of this plant to trap radicals, it can be considered as a source of natural antioxidants. Keywords: Artemisia mesatlantica maire, Antioxidant activity, Polyphenols, Flavonoids, Tannins.

P084. Use of antibiotics for managing bacterial contamination and resistance in date palm tissue-culture media

Houria Ben-Amar ${ }^{*}$, Reda Meziani, and Adil Essarioui 
National Institute for Agricultural Research, Regional Center of Errachidia, Morocco. ${ }^{*}$ Corresponding and Presenting Author: Houriabenamar@hotmail.fr.

\section{Abstract}

Date palm industry in morocco relies on tissueculture techniques for large scale production of plant material. Unfortunately, bacterial contaminations of juvenile tissues constrain the improvement of palm micropropagation rate. Antibiotics have been classically used to manage undesired bacterial growth in plant culture media. This can lead to resistance accumulation within contaminant populations if antibiotics are not properly used. In order to develop methods for managing bacterial contaminations and antibiotic resistance during date palm in vitro multiplication, we studied 9 clinical antibiotics for their inhibitory effect against 2 bacterial strains frequently associated with date palm juvenile tissues. Antibiotics differed in their effect on the two bacterial isolates. One strain was sensitive to all antibiotics with an inhibition intensity ranging from $6 \%$ to $59 \%$. However, the second isolate was completely resistant to 3 antibiotics and weakly sensitive to the others, with an intensity of inhibition smaller than $12 \%$. This study highlights the importance of alternate use of multiple antibiotics for controlling bacterial growth and managing antibiotic resistance in date palm tissue-culture media. Keywords: date palm, tissue-culture, bacterial contamination, antibiotics.

P085. Physiological analyses of limited transpiration rate under high evaporative demand during durum wheat domestication

Rachdad Fatima Ezzahra ${ }^{1,2^{*}}$, Danguy des deserts Alice ${ }^{1,3}$, El Bouhmadi Keltoum², Ghanem Michel Edmond $^{1 \S}$

1 Crop Physiology Lab, International Center for Agricultural Research in the Dry Areas (ICARDA), Rabat, Morocco; ${ }^{2}$ Departement of Biology, Faculty of Sciences Ben M'Sik, University Hassan II, Casablanca, Morocco; ${ }^{3}$ Montpellier SupAgro, France. "Presenting Author: rachdad.fz@gmail.com. ${ }^{\S}$ Corresponding author: M.Ghanem@cgiar.org.

\section{Abstract}

To satisfy increasing wheat demands, scaling up wheat production will require boosting yield in suboptimal, drought-prone areas. This increase will be particularly challenging since in several major agricultural regions of the world, yield stagnation has been observed during last decades. In addition to soil drought, drought-prone environments tend to exhibit high levels of atmospheric vapor pressure deficit (VPD), which is an additional driver of crop water loss via transpiration (TR). Recently, it was hypothesized that "water-saving" cultivars displaying TR decreases in response to high VPD, or in response to the soil drying, should lead to enhanced drought tolerance under terminal drought conditions by increasing the availability of stored soil moisture during the critical grain filling phase (Sinclair, 2017). It is likely that during breeding, breeders favored high-yielding but more water-consuming genotypes, and disfavored / excluded low-yielding but more conservative species. For this purpose, we have examined the evolution of TR traits in a domestication series of durum wheat. TR response to an artificial increase in vapor pressure deficit (VPD) of 3 species was evaluated: Triticum turgidum L. subsp. Dicoccoides, Triticum turgidum subsp. Dicoccon, and modern elite lines of durum wheat (Triticum durum Desf.). Our findings reveal a large diversity of responses especially in wild and primitive wheat. Such considerations will be particularly useful to breeding and introgression of useful traits from crop wild relatives. Keywords: Domestication, Drought, VPD, Transpiration, Water conservation traits.

P086. Diagnosis of clinical cases of infectious bursal disease using a developed rapid Taq Man-MGB real-time RT-PCR assay

Maryame Cheggag ${ }^{1 *}$, Khalil Zro², Ghizlane Sebbar ${ }^{2}$, Mohamed Mouahid ${ }^{3}$, Mohammed El Houadfi1, and Faouzi Kichou'

${ }^{1}$ Institute of Agronomy and Veterinary Medicine Hassan II, B.P. 6202, Madinat Al Irfane, Rabat, Morocco; ${ }^{2}$ BIOPHARMA, Rabat, Morocco; ${ }^{3}$ Veterinary Clinic Temara, Morocco. "Corresponding and Presenting Author: cheggagmaryame@gmail.com. 


\section{Abstract}

Infectious bursal disease (IBD) is an important contagious viral infection of immune system of poultry. Despite various types of commercially available vaccines against IBDV, this infection poses a permanent threat to the profitability of poultry industry worldwide. The aim of this work was to modify and to develop TaqMan-MGB rRT-PCR in one-step involving two fluorogenic TaqMan labeled probe for the detection of infectious bursal disease virus (IBDV) collected from suspected cases distributed in different regions of the country during the period 2013-2016. The method developed was validated for specificity, linearity, repeatability, sensitivity, reproducibility. It allowed reducing the test running time by 6 folds. This method was applied on 102 pools of bursa of fabricius (BF) samples collected from affected broiler farms suspected to be infected by IBDV. Birds showing macroscopic lesions including muscle petechial hemorrhages, hypertrophy and hemorrhage of $\mathrm{BF}$, were subjected to molecular analysis using modified protocol "TaqMan-MGB real-time RT-PCR". The validation satisfied all criteria and the assay developed could be a useful tool fora very rapid diagnosis of IBDV and permit to detect and to discriminate in one-step very virulent (vv) from non-vv (classic and variant) IBDV strains in poultry. Out of 84 IBDV positive samples, a prevalence of $33 \%$ for hyper-virulent strains and $50 \%$ for classical strains was noted. These results indicate that despite the vaccination hyper-virulent form of IBDV continues to cause serious problems for Moroccan broiler chickens. Keywords: IBDV, One-step rRT-PCR, TaqMan-MGB, Macroscopic lesions.

P087. Synergistic Effects between Exogenous Salicylic Acid and Arbuscular Mycorrhizal Fungi in date palm (Phoenix dactylifera L.) Seedlings under drought Stress

Benhiba Laila ${ }^{*}$, Abdellatif Essahibi, and Ahmed Qaddoury

Unit of Biotechnology and Symbiosis Agro-Physiology, Faculty of Sciences and Techniques, Cadi Ayyad University, Marrakesh, Morocco. "Corresponding and Presenting Author: Ibenhiba.doc@gmail.com.

\section{Abstract}

In Morocco, country traditionally producer and consumer of date, the phoenicicole sector is suffering from serious biotic and abiotic constraints such as drought, salinity and Bayoud (vascular fusariosis). However, the arbuscular mycorrhizal (AM) symbiosis has been shown to improve plant growth, mineral uptake ability and tolerance to drought stress. It also induces resistance against soil pathogens. In addition, salicylic acid (SA) or orthohydroxy benzomethylic acid is a phenolic compound which influences various physiological processes and biochemical interactions in plants. Indeed, this molecule has a regulatory role in a range of diverse physiological processes in plants, including photosynthesis, ion uptake and transport, chlorophyll synthesis, seed germination, membrane permeability and plant growth (Aftab et al., 2010). Salicylic acid influences also plant tolerance to drought (Azooz and Youssef, 2010), salinity (Gunes et al., 2007) and heavy metal stress (Moussa and El-Gamal, 2010). The aim of this study was to determine the combined effects of mycorrhizal symbiosis and salicylic acid application $(1 \mathrm{mM})$ on the growth and development of date palm (Phoenix dactylifera L.) seedlings in greenhouse conditions under different drought stress (DS) levels. Results showed that both AMF-inoculation and Salicylic-Acid can increase date palm tolerance to drought. The combination of the two factors further promoted drought tolerance, indicated by improving plants morphological parameters (leaf and root length, surface area, shoot and root fresh and dry weights) compared to control plants and increasing proline and total soluble sugar accumulation in the leaves of stressed plants. Keywords: Date palm (Phoenix dactylifera L.), Arbuscular mycorrhizal fungi, Symbiosis, Drought stress, Exogenous Salicylic acid (SA).

\section{P088. Morphological and molecular characterization of Fusarium spp. associated with Dry Root Rot disease of Citrus in Morocco}

Said Ezrari ${ }^{1,2^{*}}$, Rachid Lahlali ${ }^{1 *}$, Abdessalem Tahiri $^{1}$, Adil Asfeers ${ }^{3}$, Abdelhadi Ait Houssa ${ }^{3}$, and Abderrahim Lazraq ${ }^{2}$

1 Department of Plant Protection, Phytopathology Unit, Ecole Nationale d'Agriculture de Meknès, BPS 
40, Meknès, Morocco; ${ }^{2}$ Laboratory of Functional Ecology and Environment, Sidi Mohamed Ben Abdellah University, Po. Box 2202, Route d'Imouzzer, Fez, Morocco; ${ }^{3}$ Louata Field -Providence Verte group: PO Box ${ }^{4}$ Sefrou Region, Morocco. ${ }^{*}$ Corresponding and Presenting Author: rlahlali@enameknes.ac.ma.

\section{Abstract}

The Dry root rot (DRR) is one of a devastating disease of citrus plantations with higher incidence under biotic or abiotic stresses. It might cause significant damage to younger citrus plantations depending on the rootstock. Recently, this disease emerged as serious threat to Moroccan citrus plantations; the wilting of young citrus trees was observed in different orchards with typical symptoms of vascular necrosis and root rot. Furthermore, the diseased rootstocks displayed also a brown coloration in the stem. This disease is reported to be caused by a complex of different species of Fusarium, but the Fusarium solani was emphasized to be predominant. Despite the occurrence of this disease in the past, there was a lack of informations about the identity of the fungal pathogens involved, the favorable conditions for their development as well as appropriate strategies for their control. Therefore, this study aimed at identifying and phenotyping the fungal pathogens associated with this disease from different diseased plants and soil collected from citrus orchards and nurseries. The preliminary results underlined that the fungal pathogen responsible of dry root rot of citrus belongs to the genus of Fusarium spp. based on morphological traits and molecular characterization. As a result, a depth study of morphological and molecular characterization of Fusarium isolates is further considered in more details. Keywords: Citrus, dry root rot, Fusarium spp., morphological characters, molecular tests.

P089. Evaluation of some new introduced hybrids of sunflower (Helianthus annuus L.) under drought conditions in Morocco

Rajae Kettani ${ }^{*}$, Abdelghani Nabloussi ${ }^{2}$, Lahcen Hssaini $^{2}$, and Chems Doha Khalfi ${ }^{1}$

' Research Unit of Agronomy and Plant Physiology, National Institute of Agricultural Research (INRA), Regional Center of Meknes, Po. Box 578, Meknes, Mo- rocco; ${ }^{2}$ Research Unit of Plant Breeding and Plant Genetic Resources Conservation, National Institute of Agricultural Research (INRA), Regional Center of Meknes, Po. Box 578, Meknes, Morocco. "Corresponding and Presenting Author: r_kettani@yahoo.fr.

\section{Abstract}

Drought was always present in Morocco's history. Its importance as a structural element of the country's climate increased during the last decades, with a net reduction in the precipitation and an increasing temperature trend, as a result of climate change. The present study was carried out in Meknes area (Morocco) in order to characterize and evaluate new sunflower hybrids for their agronomic performance and tolerance to water stress often occurring at bud and flowering stage. Seven introduced hybrids were conducted in Ain Jemaa under rainfed conditions during 2016-2017. Agronomic parameters, namely plant height, head diameter, number of seeds/head, 1000-seed weight, seeds weight/head, and seed yield/ha, as well as physiological traits, like as chlorophyll level, stomatal conductance and relative water content, were measured. Seed yield was shown to be significantly correlated with number of seeds per head, head diameter and plant height. The highest seed yield, $2.84 \mathrm{t} / \mathrm{ha}$, was observed for the variety 'Laila', while the lowest yield is recorded for the variety 'Merida'. In 'Laila' hybrid, during flowering stage, there was a significant and strong positive correlation between stomatal conductance and seed yield. As flowering process was happening under drought conditions, it could be suggested that that hybrid was able to maintain open stomata and thus to yield better than the other studied hybrids. Keywords: Sunflower, hybrid, drought, seed yield, stomatal conductance.

P090. Effect of commercial organic fertilizers on soil fertility, nitrogen use efficiency and yield of broccoli grown under irrigated arid conditions in south of Morocco

Kaoutar Aouass ${ }^{*}$, Lahcen Kenny ${ }^{1}$, and Mariam Bouchfar $^{2}$

1 Department of Horticulture, Complex of Horticulture, Agronomic and Veterinary Medicine Hassan II Institute, Rabat, Morcco; ${ }^{2}$ Department of biology, 
University Ibn Zohr, Agadir, Morcco. "Corresponding and Presenting Author: aouass.kaoutar@gmail.com.

\section{Abstract}

A field research was conducted in order to determine the effect of three different types of commercial organic fertilizers (COF) as compared to compost on soil fertility, nitrogen use efficiency, growth and yield of broccoli in the Souss Massa area in south of Morocco. Broccoli seedling were raised under greenhouse and transplanted to sandy-loamy soils in open field prior to adding $200 \mathrm{~kg} / \mathrm{ha}$ of $\mathrm{N}, 80 \mathrm{~kg} / \mathrm{ha}$ of $P$ and 180 $\mathrm{kg} / \mathrm{ha}$ of $\mathrm{K}$ either as compost $(2.5,3,2.5)$, a ternary COF ( 7-4-7), a mixture of one binary COF (8-12-0) and one simple COF (0-0-30) or a mixture of three simple COF (10-0-0, 0-18-0 and 0-0-30). The highest yields (30 $\mathrm{T} / \mathrm{ha})$ were obtained with compost and the ternary COF $(28 \mathrm{~T} / \mathrm{Ha})$. As for soil fertility parameters, a clear effect of organic inputs was registered on soil organic matter, electric conductivity and $\mathrm{pH}$. The highest coefficient of nitrogen use efficiency (NUE) was obtained with the ternary COF $(64 \%)$ and the lowest $(25 \%)$ with the simple COF. Based on these findings, recommendations on how to build an organic fertilization program for leafy vegetables are discussed. Keywords: Organic fertilizer, compost, broccoli, nitrogen use efficiency, arid conditions.

\section{P091. Variations in the proline, glucose and protein con-} tents in Origanum majorana L. grown saline conditions

Hamza Elhaddaii ${ }^{*}$, Moustapha Akodad', Abdelmajid Moumen', Ali Skalli', and Mourad Baghour ${ }^{1}$

- Observatoire de la lagune de Marchica de Nador et Région Limitrophes (Labo. OLMAN-RL), Faculté Pluridisciplinaire de Nador, Université Mohamed Premier, BP 300, 62700, Selouane, Nador, Morocco. *Corresponding and Presenting Author: hamza.elhaddaji@gmail.com.

\section{Abstract}

In recent years, low rainfall in north-eastern Morocco has led to low water levels of water stored in dams. This leads the farmers to look for other alternatives to develop their agriculture, either by looking for other sources of water supply such as pumping ground- water, which is characterized in most cases by poor quality due to high salinity levels, or the introduction of other crops more resistant to salinity and with less water requirements. The objective of this study is to determine the effect of salt stress on the protein, proline and glucose contents in marjoram (Origanum majorana $\mathrm{L})$. We started our study by applying different concentrations of $\mathrm{NaCl}(0,50,100$ and $150 \mathrm{mM})$ for 44 days on plants grown in greenhouse at the Multidisciplinary Faculty of Nador - FPN. Different physiological and biochemical parameters were analyzed to evaluate the salinity tolerance of marjoram plants. Our results showed that proteins, glucose and proline contents were affected positively by different concentrations of $\mathrm{NaCl}$, reaching high value of these parameters with high $\mathrm{NaCl}$ level $(150 \mathrm{mM})$. Keywords: Salt stress, protein, proline, glucose, marjoram.

\section{P092. Improving Tomato Fruit Production and Quality by Co-Expressing LeNHX2 and SISOS2}

Mostapha Maach ${ }^{1 *}$, María Pilar Rodriguez Rosales², Kees Venema ${ }^{2}$, Fancisco Javier Gálvez ${ }^{2}$, María Elena Sánchez Romero², Mustapha Akodad', Adelmajid Moumen', and Mourad Baghour ${ }^{1}$

' Observatoire de la Lagune de Marchica de Nador et Région Limitrophes (OLMAN-RL), Faculté Pluridisciplinaire de Nador, Université Mohamed Premier, BP 300, Séloune 62700, Nador, Morocco ; ${ }^{2}$ Departamento de Bioquímica, Biología Celular y Molecular de Plantas. Estación Experimental del Zaidín, CSIC, Calle Profesor Albareda, 1, 18008 Granada, Spain. 'Corresponding and Presenting Author: mostaphamaach12@gmail.com.

\section{Abstract}

Salinity is a major environmental factor limiting crop productivity and quality especially in arid and semiarid regions. The presence of salt in the medium impose impose both an ionic and an osmotic stress to most plants and negatively affect plant growth by reducing the uptake of essential ions, such as potassium. The transporters responsible for $\mathrm{K}+/ \mathrm{Na}+$ homeostasis as well as the proteins involved in their regulation are the main mechanisms of salt tolerance. The salt tolerance locus SOS1 from Arabidopsis has been shown to encode a plasma membrane $\mathrm{Na}+\mathrm{H}+$ antiporter. 
SOS 1 activity is regulated by a complex composed of the SOS2 kinase and the SOS3 $\mathrm{Ca} 2+$ binding protein. On the other hand, NHX genes in plants encode intracellular Cation/ $\mathrm{H}+$ antiporters regulating $\mathrm{Na}+, \mathrm{K}+$ and $\mathrm{H}+$ levels in cytoplasm and intracellular compartments, providing osmotic and ionic protection. In this study, transgenic tomato plants (cv. MicroTom) expressing both the endosomal antiporter LeNHX2 and the protein kinase SISOS2 were produced. Our results suggest that co-expression of LeNHX and SISOS2 genes significantly improved growth, yield, fruit quality and enhanced salt tolerance of tomato plants growing under saline conditions. Keywords: LeNHX2, SISOS2, Co-expression, Salt stress, Tomato, Yield

P093. Micropropagation of date palm (Phoenix dactylifera L.) cv. Mejhoul: Morphological and physiological characteristics of regenerant plantlets respond to culture medium texture and composition

Reda Meziani ${ }^{1 *}$, Mouaad Amine Mazri², Essarioui Adil $^{1}$, and Fatima Jaiti ${ }^{3}$

${ }^{1}$ National Institute for Agricultural Research, Regional Center of Errachidia, Morocco; ${ }^{2}$ National Institute for Agricultural Research, Regional Center of Marrakech- Morocco; ${ }^{3}$ University Moulay Ismail, Faculté des Sciences et Techniques d' Errachidia-Morocco. "Corresponding and Presenting Author: redameziani@ yahoo.fr.

\section{Abstract}

Date palm (Phoenix dactylifera L.) has long been a major fruit crop in southern Morocco. In these areas, date production is an important source of food and income for local populations and plays a key economic role by creating suitable microclimate for growing other crops. Unfortunately, over the last century, Morocco has lost about 12 million palm trees mainly because of bayoud, a severe wilt disease caused by the fungal pathogen Fusarium oxysporum $f$. sp. albedinis. A few years ago, a strategic program was launched to restore disease-devastated areas and create new modern plantations outside traditional oases. The expected total number of palms to plant by 2020 is 3 million trees. The use of in vitro multiplication techniques is the best way to achieve this goal. Among the techniques used for date palm micropropagation, organogenesis allows for the production of true-totype plantlets. However, the efficiency of this technique depends widely on the genotype and medium composition. The effects of various culture conditions on shoot elongation, rooting, plantletphysiological characteristics, and acclimatization were studied. Adventitious shoots obtained through direct organogenesis of date palm cv. Mejhoul were used as explants. The effects of medium texture (liquid or semi-solid), polyvinylpyrrolidone (PVP), adenine, myo-inositol, and L-glutamine on plantlet quality and acclimatization were evaluated. The most effective culture medium was semi-solid half-strength Murashige and Skoog medium (MS/2). In this medium, average shoot length reached $13.15 \mathrm{~cm}$, the mean number of roots per shoot was 3.3, average root length extended to $3.15 \mathrm{~cm}$, and acclimatization success rate attained 85 $\%$. Liquid medium, PVP, adenine, myo-inositol and Lglutamine did not improve survival rate during acclimatization. Some physiological characteristics of the plantlets were also determined. Chlorophyll content and fluorescence, foliar surface and stomatal conductance were measured after 3 months of culturing in each medium. The ranges were as follows: Chlorophyll content, 12.3-31.8 CCl; chlorophyll fluorescence, 0.633-0.795; foliar surface, $7.35-13.03 \mathrm{~cm} 2$; and stomatal conductance, $10.5-34.6 \mathrm{mmol} / \mathrm{m}^{2} / \mathrm{s}$. There was a positive correlation between physiological characteristics and acclimatization success rate. These findings provide valuable information for costeffective large-scale production of date palm cv. Mejhoul plants. Keywords: Date palm, micropropagation, organogenesis, morphological characteristics, physiological characteristics.

\section{P094. Efficiency of Hedysarum flexuosum L. under stress environments}

Anass El Yemlahi", Abdelhay Arakrak, Amin Laglaoui', and Mohammed Bakkali

Equipe de Recherche en Biotechnologies et Génie des Biomolécules (ERBGB), Faculté des Sciences et Techniques de Tanger, Morocco. "Corresponding and Presenting Author: ELyemlahi@hotmail.fr

\section{Abstract}

The success of such symbiosis between legume and 
rhizobium may require the selection of both partners that have the ability to survive at various environmental conditions. In order to study the rhizobium-Sulla efficiency under environmental constraints, we investigated the diversity of 36 isolates nodulating four Hedysarum flexuosum L. known as Sulla harvested from different environmental habitats. The isolates were tested for their tolerances to extremes of temperature, $\mathrm{pH}$, salt, water deficit low, resistance to heavy metals, antibiotics, in addition to their ability to use different source of carbon and nitrogen. Correspondingly, edaphic and climatic conditions at each harvested site were analyzed. A different pattern was observed between sampled Sulla, estimated on the basis of plant growth, osmolytes accumulation, nodulation and subsequent nitrogen fixation depending on the most prevailing factors of their natural habitat and the degree of tolerance of their associated symbionts to stress-testing conditions. Results suggest that such rhizobiums could operate interdependently and/ or synergistically to detoxify the stress molecules and alleviate therefore the effects of environments stress factors. Key words: sulla, rhizobium, biological nitrogen fixation and environments stress factors.

P095. Optimization of the extraction technique for the determination of bioactive compounds in two aromatic and medicinal wild plants: Matricaria chamomilla $L$. (wild chamomile) and Cladanthus mixtus L. Oberpr. and Vogt. (Moroccan Chamomile)

Amina El Mihyaoui ${ }^{1,2^{*}}$, Maria-Emilia Candela Castillo ${ }^{2}$, Maria-Emilia Requena Candela ${ }^{2}$, and Ahmed Lamarti $^{1}$

' Laboratory of Plant Biotechnology, Biology Department, Faculty of Sciences, Abdelmalek Essaadi University, Tetouan, Morocco; ${ }^{2}$ Phytopathology Laboratory, Department of Plant Biology, Faculty of Biology, University of Murcia, Campus de Espinardo, 30100 Espinardo, Murcia, Spain. 'Corresponding and Presenting Author: elmihyaoui.amina@gmail.com.

\section{Abstract}

Medicinal plant extracts are widely used in pharmaceutical and cosmetic products. Matricaria chamomilla L, (German Chamomile, Matricaria recutita L., Matricaria suaveolens $\mathrm{L}$. chamomile) is a widely recognized medicinal plant from the Asteraceae family, it is native to southern and eastern Europe and cultivated also in countries of America and Asia, also, it is one of the most popular medicinal plants commonly used in herbal tea, for food purposes and in folk medicine. The dried flowers of chamomile contain many terpenoids and flavonoids contributing to its medicinal properties. Wild chamomile. Cladanthus mixtus is an endemic plant of Morocco, also known commercially Chamomile of Morocco, is a biennial chamenyphous Asteraceae with many upright stems. In Morocco, it is encountered in two different zones, the first between Tangier, Ouezzane, Souk Larbaa, Moulay Bousselham and Azilah, and the second between Kenitra, Sidi Slimane, Khemisset and Rabat. The extracts produced with the different solvents were determined using HPLC/DAD and HPLC/MS. In the present study, we have determined the extraction solvent medium to obtain a well-illustrated chromatogram for the purpose of identifying bioactive compounds in the flowers of two medicinal plants: Matricaria chamomilla and Cladanthus mixtus. Keywords: Matricaria chamomilla, Cladanthus mixtus, HPLC/DAD, HPLC/MS.

P096. Potential of Biocontrol in vivo and in vitro of Bacillus amyloliquefaciens and Trichoderma harzianum against strawberry anthracnose caused by Colletotrichum acutatum

Rababe Es-Soufi', Mohammed L'Bachir El Kbiach, and Ahmed Lamarti

Plant Biotechnology team, Department of Biology, faculty of sciences, Abdelmalek Essaâdi University, Morocco. "Corresponding and Presenting Author: rababeessoufi@gmail.com.

\section{Abstract}

Anthracnose is one of the most widely known strawberry diseases; it can be destructive to strawberry plants, it is caused by Colletotrichum species and mainly by Colletotrichum acutatum. The pathogen produced lesions on leaves, fruit and other parts of plant. The control of fungal diseases attacking strawberry plants is mainly done by treatment of the soil and the plants using chemical pesticides. But still a lot of work needs to be done before any environmentally friendly and consistent control strategies come 
into existence. The use of the antagonists was done with a lot of procedures starting by with isolation and finishing by selecting strains highly effective for the Biocontrol. Relatively little is known about the exact mechanisms used by Bacillus amyloliquefaciens in its behavior as a biocontrol agent on plants. Here, the suspensions of Bacillus amyloliquefaciens and Trichoderma harzianum at concentration of $3.105 \mathrm{UFC} / \mathrm{ml}$ and 104 spores $/ \mathrm{ml}$ respectively (previously selected as the minimal inhibitory concentrations of Colletotrichum acutatum development) were tested in vitro and in vivo against Colletotrichum acutatum. In vitro study: The wounded Strawberry's leaves were sprayed with the antagonist suspension and inoculated one hour later by Colletotrichum acutatum, then held in moist chambers at $25^{\circ} \mathrm{C} \pm 2$. The leaves did not become infected with $C$. acutatum. In vivo study: the strawberry plants were sprayed with the mixture of the antagonist and pathogen suspensions, then held in moist chambers at $25^{\circ} \mathrm{C} \pm 2$. The plants didn't get anthracnose symptoms. The concentrations studied were effective to control the strawberry anthracnose in vivo and in vitro, next we will start the study in field for this application. Keywords: Biocontrol, Antagonism, Anthracnose, Bacillus amyloliquefaciens, Trichoderma harzianum.

\section{P097. Germination of somatic embryos derived from Moroccan Quercus suber $L$. trees}

Safâ̂ Rahmouni", Mohamed L'bachir El Kbiach, and Ahmed Lamarti

Laboratory of Plant Biotechnology, Department of Biology, Faculty of Sciences, Abdelmalek Essaadi University, M'hannech II, B.P. 2121 , Tetouan, Morocco. ${ }^{*}$ Corresponding and Presenting Author: safaarahmouni@hotmail.fr.

\section{Abstract}

Cork oak (Quercus suber L., Fagaceae) is one of the most important species of the mediterranean basin due to its ecological and socio-economical interests. Nevertheless, the increasing demand for cork and the low natural regeneration provides lost of cork oak forest. Vegetative propagation by in vitro methods (techniques of micropropagation) presents many advantages compared to traditional methods. Somatic embryogenesis is considered as the best method of micropropagation that can solve problems of yield and intraclonal variability. It had several successes in forest species allowing the production of trees from somatic embryos. The goal of our study is the regeneration of seedlings from Moroccan cork oak somatic embryos of three lines (Ain Rami 1, Ain Rami 2 and Bouhachem). To this end, we were interested in studying the effect of growth regulators, embryos size and the period of cold storage on the germination of cork oak somatic embryos. We noticed the presence of different types of shoots (Shoots of normal size with or without roots, shoots of small size with or without roots and abnormal shoots). The frequency of germination, the mean number of shoots per embryos, the mean length of shoots, the mean number of leaves per shoot and the mean number of roots per shoot depend on the genotype and the plant growth regulator used. Germination of large somatic embryos (>12 mm and with large cotyledons) from the three embryogenic lines was successful. Stratification at $4^{\circ} \mathrm{C}$ for 2 months was the most efficient for stimulating somatic embryos germination of Quercus suber L. Keywords: cork oak, Quercus suber L., Fagaceae, secondary somatic embryogenesis, germination.

\section{P098. Effect of 6-Benzylaminopurine combined with different auxins on vegetative micropagation of Origa- num compactum $L$.}

\section{Rajae Benkaddour ${ }^{*}$ and Ahmed Lamarti}

Laboratory of Plant Biotechnology, Department of Biology, Faculty of Sciences, Abdelmalek Essaadi University, M'hannech II, B.P.2 121 , Tetouan, Morocco. ${ }^{*}$ Corresponding and Presenting Author: Benkaddourrajae27@gmail.com.

\section{Abstract}

Morocco is well known by botanical diversity of medicinal and aromatic plants that show a socioeconomical interest. Indeed, the medicinal and aromatic plants have constituted recently an important choice in various utilizations such as cosmetic, food and therapeutic fields. However, the anarchic collect of some endemic plants could constitute a main reason for their disparition; otherwise the research of alternatives ways by in vitro culture of these species is a promising strat- 
egy in biotechnology. In this context, our work rely on (i) improvement, valorization, conservation and characterization of medicinal and aromatic plants, already propagated in the north of Morocco, (ii) production development by in vitro culture of four selected species belong to Origanum genus (Origanum majorana L., Origanum vulgare L. Origanum elongatum L.Origanum compactum L.) in order to answer the farmers needs and the introduction of an alternative culture of oregano because of a high value added. A part of my research work aimed to obtain in vitro plants of Origanum compactum L. with a good quality and to achieve its acclimatization by realizing different tests. First of all, we determined the most suitable mineral medium, afterward, realized the test of cytokinins to know the one that gives the best results in terms of budding, growth, hyperhydricity, regeneration and rooting. The best one was the 6-Benzylaminopurine but the length of the root wasn't good enough to succeed the acclimatization. To obtain the explants of Origanum compactum L. having an important length of roots and then to success its acclimatization; We have tested four auxins (Indole-3-acetic acid, Naphtalene acetic acid, Indole butyric acid, 2,4-Dichlorophenoxy acetic acid.) each of them have been added to the medium at four concentrations $(0,2 \mathrm{mg} / \mathrm{I}-0,5 \mathrm{mg} / \mathrm{I}-0,8 \mathrm{mg} / \mathrm{I}$ and $1 \mathrm{mg} / \mathrm{I})$ in the presence of $0,5 \mathrm{mg} / \mathrm{I}$ of 6 -Benzylaminopurine. Our results show that the rooting and the acclimatization were the best when the combination between $1 \mathrm{mg} / \mathrm{I}$ of Indole3-acetic acid and $0,5 \mathrm{mg} / \mathrm{l}$ of 6-Benzylaminopurine was applied. Our current work is about the study of other growth factors on the budding, growth, hyperhydricity, regeneration, rooting and acclimatization of Origanum compactum L. Keywords: Aromatic and medicinal plants, Origanum,phytohormones, auxins.

\section{P099. Identification of the Cœlomic Microbial Commu-} nity of Lumbricus terrestris (Annelida: Lumbricidae)

Lamia Yakkou*, Maryam Chelkha, Mohammed Raouane, Souad Amghar, and Abdellatif El Harti.

Lumbricidae, Improving Soil Productivity and Environment (LAPSE), Centre " Eau, Ressources Naturelles, Environnement et Développement Durable (CERN2D) " Mohammed $V$ University in Rabat, Ecole Normale Supérieure (ENS). Avenue Med Belhassan El Ouazani. BP 51 18, Takaddoum-Rabat, Morocco. "Correspond- ing and Presenting Author: Yakkou.lam@gmail.com

\section{Abstract}

Earthworms play an important role in soil fertilization. These annelids interact with soil microorganisms to promote decomposition, mineralization and recycling of the organic material. Several studies have focused on the study of microorganisms associated with the earthworm's digestive tract. In the present work, however, we were interested in the microorganisms possibly associated with the worm at the level of the coelomic liquid. In fact, microscopic examination of coelomic fluid smears taken by puncture of the coelomic cavity of Lumbricus terrestris revealed the presence of microorganisms of various shapes and sizes. Isolation and culturing revealed the presence of three bacterial strains. Phenotypic and genotypic studies (PCR amplification of $16 \mathrm{~S}$ rDNA) allowed the identification of two of the bacterial strains: Pseudomonas fluorescens and Buttiauxella ferragutiae. The third strain, which is not listed in the NCBI database, may be a new bacterial species. The two bacterial strains that were identified have a proven ecological and agronomic interest: Pseudomonas fluorescens, a rhizosphere-secreting bacterium that secretes indole-3-acetic acid (phytohormone), plays an important role in protecting and promoting plant growth. Buttiauxella ferragutiae, on the other hand, is very little studied with regard to its activities at ground level. However, this strain has already been isolated from the intestine of the earthworm. Keywords: Lumbricus terrestris, Coelomic fluid, microorganisms, PCR16S DNA, AIA, Soil productivity, PGPR.

\section{P100. Screening of phosphate solubilizing rhizobia iso- lated from Hedysarum nodules in North of Morocco}

Samia Hamane ${ }^{1 *}$, Saida Aarab ${ }^{1,3}$, Mounir Hassani Zerrouk ${ }^{2}$, Anas El Yemlahi', Amin Laglaoui', Mohammed Bakkali', and Abdelhay Arakrak'

1 Equipe de Recherche de Biotechnologies et Génie des Biomolécules (ERBGB), Faculté des Sciences et Techniques de Tanger B.P.: 416 - Tanger, Morocco; ${ }^{2}$ Faculté polydisciplinaire de Larache, B.P.: 745 - Larache, Morocco; ${ }^{3}$ Faculté des Sciences et Techniques d'Al Hoceima, B.P.34, Ajdir Al Hoceima, Morocco. ${ }^{*}$ Corresponding and Presenting Author: samia- 
hamane@gmail.com.

\section{Abstract}

Plant-microorganisms' interactions in the rhizosphere are the major determinants of plant productivity and soil fertility. The use of beneficial bacteria as biological alternative for chemical inputs needs the selection of competent rhizobacteria exhibiting plant growth promoting (PGP) attributes. This study was aimed to select phosphate solubilizing rhizobia strains isolated from root nodules of the legume Hydesarum flexuosum grown in North Morocco, and to evaluate their antagonistic ability against Fusarium oxysporum and Aspergillus ochraceus. Out of 11 isolates, 7 bacteria were able to solubilize tricalcium phosphate (TCP) that were evaluated for more PGP activities. As results, none of these selected bacteria proved to be able to synthesize hydrogen cyanide $(\mathrm{HCN})$, while the amounts of indole acetic acid (IAA) produced by these bacteria ranged between $0.27 \mathrm{mg} 1-1$ and 1.51 mg l-1. Besides, only 3 strains presented a positive profile for siderophores production. As for extra cellular enzymes production (urease, cellulase, amylase, protease and chitinase), the percentage of isolates that produced these enzymes was respectively: $0 \%$, $100 \%, 28 \%, 100 \%$ and $85 \%$. A quantitative assay of TCP solubilization was conducted too, and solubilized $P$ concentrations were between $52 \mathrm{mg} / \mathrm{l}$ and $81 \mathrm{mg} / \mathrm{l}$, accompanied by a drop-in media $\mathrm{pH}$. Since the bacterial enzyme ACC-deaminase plays a significant role in sustaining plant growth under abiotic stress conditions, 5 rhizobia were capable to use ACC as the sole nitrogen source. This study reveals the potential of sometest rhizobia to be used as efficient bio-inoculants. Keywords: Hydesarum flexuosum, Rhizobia, Fusarium oxysporum, Aspergillus ochraceus, PGP, TCP, Biofertilizer.

P101. Assessment of Soil Erosion by USLE Model Using Remote Sensing and GIS - a Case Study of Moulay Bouchta Watershed, Western Rif, Morocco

A. Zouagui ${ }^{1 *}$, M. Sabir2, M. Naimi ${ }^{\prime}$, M. Chikhaoui ${ }^{1}$, and $M$. Benmansour ${ }^{3}$

'Unité de Recherche: Gestion conservatoire des eaux et des sols - IAV-Hassan II, B.P. 6202 - Instituts, 10101, Rabat, Maroc; ${ }^{2}$ École Nationale Forestière
d'Ingénieurs (ENFI), BP 511 , Tabriquet - Salé, Maroc; ${ }^{3}$ Centre National de l'Énergie, des Sciences et des Techniques Nucléaires (CNESTEN), BP 1382, R.P.10001 - Rabat, Morocco. "Corresponding and Presenting Author: zouagui.anis@gmail.com.

\section{Abstract}

Soil erosion causes many environmental and socioeconomic problems: loss of biodiversity, decrease in the productivity of agricultural land, siltation of dams and increased risk of flooding. It is therefore essential to establish a detailed evaluation of this process before any spatial planning. To evaluate the effects of soil erosion spatially and quantitatively in order to face this phenomenon, and propose the best conservation and land development strategies, the Universal Soil Loss Equation (USLE) coupled with remote sensing and geographic information system (GIS) is applied. This model is a multiplication of the five erosion factors: the erosivity of the rain, the erodibility of the soil, the inclination and the slope length, the vegetation cover and the anti-erosion practices. The study area is the Moulay Bouchta watershed (7 889 ha), which is located in the western part of the Rif Mountains, is characterized by a complex and contrasting landscape. The resulting soil loss map shows an average erosion rate of $39.5(\mathrm{t} / \mathrm{ha} / \mathrm{yr}), 87 \%$ of the basin has an erosion rate above the tolerance threshold for soil loss $(7$ (t/ha/yr)). Soil losses per subbasin range from 16.2 to 81.4 (t/ha/yr). The amount of eroded soil is estimated at $311,591(\mathrm{t} / \mathrm{yr})$, corresponding to a specific degradation of 12.1 (t/ha/ $\mathrm{yr}$ ). In the absence of any erosion control, $25 \%$ of the soil losses would reach the new dam located a little upstream of the basin outlet, reducing its water mobilization capacity to 59,625 (m3/yr). The application of Principal Component Analysis (PCA) to soil erosion factors shows a significant influence of topographic factor (LS) on soil erosion process, followed by the effect of support practices (P), then by soil erodibility (K). Keywords: Erosion, Siltation, USLE, Rif Mountains, Watershed.

P102. Differential evolution and the impact of pests on the biochemical defensive profile during ripening of five Vitis vinifera $L$. grapes varieties in the Marrakesh region

Hicham Jediyi ${ }^{*}$, Khalid Naamani ${ }^{1}$, and Abderrehim 


\section{Ait Elkoch ${ }^{1}$}

${ }^{1}$ Laboratory of Protection and Valorization of Vegetable Resources. Faculty of Sciences Semlalia Marrakech, Cadi Ayyad University, My Abdallah Street, PB: 2390, Marrakesh 40000, Morocco. *Corresponding and Presenting Author: jedhicham@gmail.com

\section{Abstract}

Vitis vinifera $(L)$ is a plant sensitive to various ravages and diseases such as pests. In this study we want to develop the evolution of the phytochemicals of the five grape varieties involving three introduced (Superior, Early sweet and Flame) and two other local varieties (Abbou and Adari) during the three stages of maturity (green, ripening and mature) in Oudaya of Marrakesh région. We wish to show the dynamic changes that have taken place in the clusters during the physiological changes complex issues related to the development and maturation, also the action of pests (Diptera) on the evolution of the biochemical profile of these varieties during ripening grape, especially our unknown local varieties. The parameters concerned the amount of total sugars, proteins, the activity of phenylalanine-ammonia lyase (PAL), tyrosine-ammonia lyase (TAL) and the lipid peroxydation. The analysis of total sugars and proteins showed their gradual increase during the period of ripening fruit of all varieties. We recorded in the TAL activity, a decrease in the course of the maturation of the fruit, and then we noticed an increase in the PAL activity in the ripe fruit for all varieties. This evolution of the parameters analyzed by the "ANOVA" test, showed a significant difference for all phytochemicals $(P<0.05)$ between the different varieties of vines according to the stages of maturity studied and a significant effect on the case of fruit, attacked or healthy one for all varieties $(P<0.01)$ translating a significant difference between the healthy and attacked mature vines fruit for all varieties. As regards the distribution of variables, on the basis of PCA analyze of the development stages according to variety and phytochimicals, we have observed that the sugars, proteins and tyrosine ammonia-lyase for PC2 are the most variables that have contributed to the discrimination of the grapes according to their stage of maturity and/ or varieties. Keywords: Vine varieties, Maturity grape stages, Phytochemical compounds.
P103. Selected biofertilizers effects on plant growth and yield of vegetable crops under field conditions

Abdel-llah Tahiri ${ }^{1,2^{*}}$, Zakariya Es-Salmi ${ }^{2}$, Anas RakIami $^{1,2}$, Noura Bechtaoui ${ }^{1}$, Abdelilah Meddich ${ }^{2}$, and Khalid Oufdou'

1 Laboratory of Biology and Biotechnology of Microorganisms, Faculty of Sciences Semlalia, Cadi Ayyad University, PO Box 2390, Marrakech, Morocco; ${ }^{2}$ Laboratory of Biotechnology and Plant Physiology, Faculty of Sciences Semlalia, Cadi Ayyad University, PO Box 2390, Marrakech, Morocco. "Corresponding and Presenting Author: abdelilah.tahiri.1993@ gmail.com.

\section{Abstract}

At the present time, food security is considered as one of the most serious challenges facing society which will further enhance demand on the production of global food. The current pressure on agriculture has caused land degradation, expansion into more marginal areas and soil types, and higher expectations of agricultural productivity per unit area. Therefore, reliable, environmentally friendly techniques are needed to sustainably meet growing global food demands. Some plant-microbe interactions like plant growth promoting rhizobacteria (PGPR), arbuscular mycorrhizal fungi (AMF) and composts has been known that they are widely used and enhance plant growth by different action mechanisms. In this context, the main objective of this study is to evaluate the impacts of selected biofertilizers on the plant growth and the yield of vegetable crops in field. The leek (Allium porrum L.) and lettuce (Lactuca sativa L.) plants were grown on soil with low levels of organic and mineral matter. We used bio-fertilizers based on AMF, PGPR and compost obtained from plant and animal wastes, alone or in different combinations. The biological treatments applied showed noticeable results compared to the control without bio-fertilizers. Indeed, the amendment by the compost $M 3$ based on green waste and livestock unit manure has considerably improved biomass parameters and yields of the two cultures. These increases were clearly significant compared to the other treatments, followed by AMF. Nevertheless, some other combinations of PGPR-AMF and compost are also shown to be beneficial for the improvement of 
the yield of the tested cultures. Thereby, the use of such bio-fertilizers can be an interesting strategy for the sustainable management and development. Keywords: Arbuscular mycorrhizal fungi, PGPR, compost, bio-fertilizers, yield, Allium porrum L., Lactuca sativa L., field. Acknowledgments: This work is financially supported by the project PPR-CNRST.

P104. Caracterisation of the Moroccan varieties for agronomique and quality traits for breadmaking

Kenza Eddakir ${ }^{1,2^{*}}$, A. Rochdi ${ }^{1}$, and W. Tadesse ${ }^{2}$

1 Faculty of Sciences, University Ibn Tofail, kenitra, Morocco; ${ }^{2}$ International Center for Agricultural Research in Dry Areas (ICARDA), Rabat, Morocco. "Corresponding and Presenting Author: kenza.eddakir@ hotmail.fr.

\section{Abstract}

Everywhere and because of the importance of wheat, farmers strive to maximize yield, minimize cost, reduce environmental impact and ensure a high baking quality. Seventeen of the Moroccan varieties from INRA , SONACOS and ICARDA are tested for agronomic and quality traits for two years 2016 and 2017. Yield and its component were collected at harvest. Proteins were determined using the kjeldahl Method. Glutenins were extracted from wholemeal flour and electrophoresis was carried out for one grain from each cultivar. For each of the cultivars dough strength, stability and water absorption were assessed using the Chopin Farinograph and the Mixograph, and the samples were also assessed using SDS sedimentation test. Correlations were calculated between the data obtained in these tests of functionality and the quantity of protein in the grain. Most of the values obtained in the functionality tests were correlated significantly with grain protein content. But in general, the results shows that the quality of the Moroccan varieties still very poor and many efforts should be made to improve the local quality for bread making and insure a good yield to meet the population needs and insure the self-sufficiency. Wheat breeders are strongly encouraged to overcome the challenge that face the culivation of wheat and create new varieties that would be tolerant to drought, resistant to dome diseases and with a good quality. Keywords: Triticum aestivum, gluten proteins, SDS Page, breadmaking, quality, yield.

P105. The optimal condition of storage and enthalpyentropy compensation analysis of the powder of Taraxacum Officinale's leaves in moisture sorption phenomena

Haytem Moussaoui", Idlimam Ali, and Abdelkader Lamharrar

Laboratory of Solar Energy and Medicinal Plants, Teacher's Training College, Cadi Ayyad University, BP 2400 Marrakesh, Morocco. *Corresponding and Presenting Author: haytem.moussaoui@gmail.com.

\section{Abstract}

Taraxacum Officinale is a kind of flowering plant that belongs to a vast family of Asteraceae. This family is represented by more than 2500 species. They are utilized for various purposes that include for dyspepsia, bile stimulation, bruises, rheumatism, eczema and muscle aches. Moreover, the powder of dandelion are used as special ingredients in soups, salad green, teas and wine. The Taraxacum Officinale used in this study were collected locally in Settat region, Morocco. The aim of this study is to determine the experimental sorption isotherms, the optimal water activity, and the net isosteric heat of sorption in order to conserve the powder. These isotherms are a powerful tool to know the state of water inside the product and his functional availability in the biological and chemical substances. The sorption isotherm curves were determined experimentally for the Dandelion seeds at three temperatures $\left(30,40,50{ }^{\circ} \mathrm{C}\right)$ and relative humidity within the range of $5-90 \%$ commonly used in the drying and storage. The net isosteric heats of sorption of water were determined from the equilibrium data at different temperatures. The compensation theory was further used to good straight lines were observed for adsorption and desorption. The Gibbs free energy values are positive $(\Delta G \beta>0)$, indicating that the sorption processes are not spontaneous. Keywords: Taraxacum Officinale, moisture sorption, optimal water activity, enthalpy, entropy. 
P106. Antioxidant activity of Anysophyllea boehmii seeds from Burundi

Jonathan Niyukuri", Raiti J., and Hafidi A.

Laboratory of Food Sciences of Cadi Ayyad University of Marrakech, Morocco. "Corresponding and Presenting Author: jonaniyu@gmail.com.

\section{Abstract}

The objective of this study is to valorize some indigenous trees. The samples of seeds were harvested in October at 3 sites namely: Mumirwa (1000-1500 $\mathrm{m}$ of altitude), Buragane (1200-1400 m of altitude) and Buyogoma (1400-2000 m of altitude). The fruit have a mesocarp edible, a thin pericarp and a tenders' seeds. The seeds were dried at room temperature in the laboratory. Ethanolic (80\%) extraction were performed from powder obtained by grinding the seeds. With ethanolic extract, Total Phenol Content of samples were determined using Folin-Ciocalteu Method and flavonoid contents were quantified using the colorimetric method of aluminum chloride. The antioxidant activity was evaluated by scavenging of DPPH (2, 2-diphenyl-1-picrylhydrazyl) free radical and Ferric Reducing Antioxidant Power (FRAP) Assay. The kinetics of DPPH reduction by ethanol extract and ascorbic acid were performed by determining steady state. Parameter, Time to the equilibrium state of IC50 (TEC50), allowed to calculate the Effectiveness Anti-radical (EA). The ethanolic extraction yield were $14.80 \pm 0.18 \%$ of Dry Weight (DW), Total Phenol Contents obtained were $851.89 \pm 34.96$ $\mathrm{mg}$ Gallic Acid Equivalent/100 $\mathrm{g}$ of DW and flavonoids contents were $261.35 \pm 102.45 \mathrm{mg} \mathrm{EC} / 100 \mathrm{~g}$ MS. The IC50 of Ethanolic extract, Total Phenol and Ascorbic Acid were respectively $0.080 \pm 0.012 \mathrm{mg}$ of extract $/ \mathrm{ml}, 0.0033 \pm 0.0012 \mathrm{mg}$ Gallic Acid Equivalent $/ \mathrm{ml}$ and $0.095 \pm 0.003 \mathrm{mg}$ of Ascorbic Acid/ $\mathrm{ml}$. The TEC50 of Ethanolic extract and Total Phenol was $11 \pm 2.2 \mathrm{~min}$ and for Ascorbic Acid was $5 \pm 0.6$. The Effectiveness Anti-radical of Ethanolic extract, Total Phenol and Ascorbic Acid were respectively

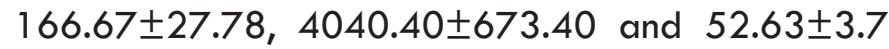
$\mathrm{ml} / \mu \mathrm{g} . \mathrm{mn}$ the reducing power of plant extracts was $3217.80 \pm 415.36 \mathrm{mg} \mathrm{AAsc} / 100 \mathrm{~g}$ de DW. These results show that of Anysophyllea boehmii has a high antioxidant activity and even greater than that of synthetic antioxidants. Thus, in light of this study, ac- tive ingredients can be isolated from Anysophyllea boehmii polyphenols food industry, cosmetic, and pharmaceutical use. Keywords: Antioxidant activity, indigenous trees, Phenol and flavonoids

\section{P 107. Traditional Phytotherapy in the High Atlas: Impor- tance and Valorization}

Salima Boutahiri ${ }^{1,2^{*}}$, Ayoub Asbabou ${ }^{1,2}$, Najia El HamzaouiSS ${ }^{1,3}$, Malika Mahjoubi ${ }^{1,2}$, Amal Bouzoubaa $^{1,2}$, Touriya Zair ${ }^{1,2 \S}$

${ }^{1}$ Research Team of Chemistry of Bioactive Molecules and the Environment, Faculty of Science, Moulay Ismail University, Meknes, B.P. 11201 , Meknes, Morocco; ${ }^{2}$ Laboratory of Materials Chemistry and Biotechnology of Natural Products, Faculty of Science, Moulay Ismail University, Meknes, B.P. 11201 Zitoune, Meknes, Morocco; 3 Laboratory of microbiology, Mohamed V Prefectural Hospital Center, Meknes, Morocco. "Presenting author: boutahirisalima@gmail.com. §Corresponding author: touria.zair@yahoo.fr.

\section{Abstract}

Herbal medication is an ancient practice. Human beings have always used plants to ensure their survival and to relieve their ills. In this sense, the objective of this work is to highlight the importance of the traditional phytotherapy and to enhance the supply of medicinal plants in the High Atlas of Morocco. For this purpose, an ethnobotanical study was carried out, and six remarkable spontaneous species were selected from the local pharmacopoeia of the region (Thymus satureioides, Mentha rotundifolia, Lavandula mairei, Pallenis spinosa, Artemisia mesatlantica and Teucrium polium) for a laboratory study (phytochemical screening, polyphenol and favonoid assays and the valuation of antioxidant activity by DPPH). The ethnobotanical study showed that $91.71 \%$ of respondents use plants $155.63 \%$ refer to their own experiences and those of their ancestors, and $36.09 \%$ resort to herbalists). $68.33 \%$ of these users are women, $50 \%$ are between 15 and 40 years old and $70 \%$ are illiterate. Plants are used for a therapeutic purpose in $86.20 \%$ of cases. This medicinal flora is widely used for the treatment of the digestive system diseases $(46.06 \%)$. The different diseases are mainly treated by decoction of the foliage, thus constituting 
the preparation method and the vegetal organ the most used. $81.65 \%$ of cases show improvement after treatment with plants. The phytochemical screening revealed the presence of tannins and flavonoids in the six-studied species and a high content of saponins for Thymus satureioides. The highest yields of total polyphenols were recorded in the species Pallenis spinose $(19.36 \%)$, Artemisia mesatlantica $(18.5 \%)$ and Teucrium polium (16.23\%). On this basis, the results for the total polyphenol assay were $2.733,2.450$ and $2.166 \mathrm{mg} \mathrm{GA}$ equivalent/g for Pallenis spinosa, Artemisia mesatlantica and Teucrium polium respectively, and the results for the flavonoid assay were 2.083, 2.041 and $1.966 \mathrm{mg}$ Quercetin equivalent/g for the same respective species. Extracts from the studied plants showed an interesting antioxidant activity. The best result was held by Teucrium polium extract, which reduced $50 \%$ of the free radical DPPH by a concentration of about $0.51 \mathrm{mg} / \mathrm{ml}$. Keywords: Traditional phytotherapy, ethnobotanical study, Thymus satureioides, Mentha rotundifolia, Lavandula mairei, Pallenis spinosa, Artemisia mesatlantica, Teucrium polium, phytochemical screening, polyphenols, flavonoids, antioxidant activity.

P108. Phenotypic and Symbiotic Characterization of Rhizobia Nodulating Lotus creticus in the North of Morocco

Taoufik Belechheb ${ }^{*}$, Mohammed Bakkali, Amin Laglaovi, and Abdelhay Arakrak

Equipe de Recherche de Biotechnologies et Génie des Biomolécules (ERBGB), Faculté des Sciences et Techniques de Tanger, Abdelmalek Essadi University, Morocco. "Corresponding and Presenting Author: taoufik.belechheb@gmail.com.

\section{Abstract}

Plants belonging to the Lotus genera are now considered to be promising legumes for sustainable development, because it was used as a pioneer plant, improving soil structure and providing symbiotic inocula to promote plant succession. Moreover, by its creeping habit, this species reduces the effects of the severe Mediterranean winds thus fulfilling a primordial function in the protection and the preservation of the ecosystems contributes to soil stabilization, provides high quality animal fodder and its productivity is important on a wide range of soils so they are increasingly utilized in pastures. Lotus sp, may play a key role in the nitrogen cycle, as it comes into symbiosis with nodulating bacteria Legumes (LNB) collectively known as rhizobium and make them excellent candidates to maintain soil cover, dune stability, restore or improve the fertility of degraded soils and use for sustainable agricultural systems. This work was conducted to evaluate and characterize the phenotypic diversity (biochemical and physiological tests) that exists in a rhizobial collection isolated from nodules of Lotus creticus collected from different sites in Tangier in the north of Morocco. Tolerance to salinity, high temperatures, acid and alkaline $\mathrm{pH}$, heavy metals and carbon and nitrogen source assimilation as well as symbiotic and cultural characteristics allowed the description of a wide physiological diversity among tested isolates. The dendrogram prepared by the UPGMA method, allowed us to highlight the most resistant isolates to tested stress conditions which represent the best candidates for the production of good quality inoculum for plants in marginal areas. Keywords: Lotus creticus , phenotypic characterization, Rhizobium, Tangier, forage.

\section{P109. Life strategy and social participation of Moroc- can youth in poor rural areas of Morocco}

\section{Noureddine Bahri ${ }^{1 *}$, A. Bentaïbi ${ }^{1}$, and T. Desrues ${ }^{2}$}

${ }^{1}$ Regional Center of Agricultural Research of Meknes, BP. 578 Meknes VN, Morocco; ${ }^{2}$ Institute of Advanced Social Studies (IESA) Cordoba, C/ Campo Santo de los Mártires, 714004 Cordoba, Spain. *Corresponding and Presenting Author: bahrinour@yahoo.fr.

\section{Abstract}

This research is carried out about young people from the mountainous areas of the Moroccan middle Atlas to better understand their logics and strategies of life. The study was focused on three communes marked by a high rate of poverty and a pronounced distance from the main lines of communication and the political and economic decision-making centers. It has adopted a dual approach to data production, namely: the administration of a survey with more than 300 individuals (a quantitative approach) and open interviews (a 
qualitative approach) with young people in addition to different key informers. The first objective of this research concerns the exploration of the various strategies of life of young people in situations of social, economic and ecological constraints (high poverty and unemployment rates, underemployment, agriculture subject to climatic risk, lack of non-agricultural employment opportunities and lack of infrastructure for culture or recreation). The second is focused on the perception of their situation with particular attention to the analysis of the level of citizen participation of rural young people and the relationships maintained with the main actors of local development in general and of agricultural development in particular. This research postulates that these populations of young people are currently experiencing mutations and profound ruptures that challenge current traditional systems. The identification of these mutations would give some visibility to the complexity of these areas and would affirm the need to incorporate this notion of diversity into the public policies in Morocco in the field of social, economic and cultural integration of rural youth. This consideration would enable young people to be better mobilized in local development activities and to reduce their dissatisfaction with their situation. This research has established that the young people of the regions studied are carrying diversity at the ideological level or values, i.e. they confront the management of their daily life in a differentiated way according to a series of axiological variables highlighted. This diversity is itself dependent on the nature and extent of the capital (economic, social, educational-cultural) available among the young people concerned. Keywords: Social change, Youth, local development, Agricultural development, Life strategy, social participation.

\section{P1 10. Introduction of new binary forage associations for crop-livestock systems under semi-arid conditions}

Rajae Kettani 1*', Chems Doha Khalfi' , and Driss Chergui $^{2}$

1 Research Unit of Agronomy and Plant Physiology, National Institute of Agricultural Research (INRA), Regional Center of Meknes, Po. Box 578, 50000 Meknes, Morocco; ${ }^{2}$ Experimental area of Douyet, Fes, Morocco. "Corresponding and Presenting Author: $r_{-}$ kettani@yahoo.fr.

\section{Abstract}

The improvement of forage protein autonomy of farms in semi-arid regions implies the increase of the share of adapted and productive associations fodder as legume and cereals. Indeed, they have many agronomic advantages that must be emphasized: no need to irrigate, a balanced protein product in relation to pure cereals, better protein content, a good competitiveness against weeds and increased resistance of the mixture to diseases. The experimentation was carried out at INRA experimental station in Fes (Morocco) for three years to test new binary associations with a seeding rate of $120 \mathrm{~kg}$ per ha and the same proportions of 50-50 mixed species combined with different doses of nitrogen. The associated species are vetch Guich (INRA variety) which is early, productive in green fodder and resistant to Alternaria and Botrytis and four early to moderately precocious tutors: A rust resistant and productive Nasr oat (INRA variety); two early and high barleys: Amalo (INRA variety) and Kaws (Spain variety) and a rustic triticale Forricale, very tolerant to water deficit and pouring (French variety). The results obtained show that it is possible to improve significantly the production and quality of forage associations through a good choice of varieties in mixture and appropriate nitrogen fertilization adapted to the production objective. Kaws-Guich combined with 120 nitrogen units per ha produced 9 tons of dry matter per ha and allows a productivity increase of $45 \%$ compared to the region average obtained with local binary associations. It also triples the proportion of vetch at harvest and consequently improves forage quality, as well as an increase of dry matter content of $27 \%$ and therefore a better forage nutrient concentration. On another side, the Forricale-Guich combined with only 60 nitrogen units per ha produced 5.5 tons of dry matter per ha and offers a better stand composition with a proportion of vetch of $40 \%$ and an increase of forage dry matter content of $17 \%$ compared to the region data. These two associations harvested in the cheesy stage of grain before drought occurrence has allowed increasing the qualitative and quantitative forage. They represent an interesting response for self-sufficiency and less dependence on weather conditions and suit specifically for farms poorly suited to corn silage. Keywords: Binary forage associations, varieties, nitrogen, drought, yield, dry matter. 
P111. Cytokinins influence the microtuberization and growth of potato (Solanum tuberosum L) plantels in vitro

Aziz Akdi ${ }^{1,2 *}$, Ahlam Hamim ${ }^{1}$, Imane Saibari ${ }^{3}$, Sliman El Hani ${ }^{1}$, and Nourdin Mtili ${ }^{2}$

1 National Institute of Agricultural Research, Tangier, Morocco; ${ }^{2}$ Faculty of Sciences, AbdElmalek Essaidi University, Tetouan, Morocco; ${ }^{3}$ Faculty of Science and Techniques of Tangier, Abdelmalek Essaadi University, Morocco. "Corresponding and Presenting Author: Azizakdi99@gmail.com.

\section{Abstract}

Potato (Solanum tuberosum L.) is a very important crop in agricultural production in Morocco. However, certified seeds production still one of the major problems hindering the development of this crop. To overcome this problem, the tissue culture techniques including microtuberization are used worlwide to produce prebasic, virus-free seed potatoes know as microtubers. The microtubers are sown in a protected enviroment to produce minitubers. Tuberization is the predominant type of reproductive development for tuberous plants; it is a complex process, whih is controlled by several factors. The main objectif of the present study was to standarize the media for potato plant growth and microtuber induction on MS medium (Murashinge and Skoog, 1962) with and without cytokinins. To do this, four culture media were tested: $M S 1=M S+$ $80 \%$ sucrose; $M S 2=$ MS $/ 2+80 \%$ sucrose; $M S 3$ $=$ MS $/ 2+80 \%$ Sucrose $+4 \mathrm{mg} / \mathrm{I}$ Kinetin $+1 \mathrm{mg}$ / I BAP (6-benzylaminopurine); MS4 = MS / $2+$ $80 \%$ Sucrose $+0.2 \mathrm{mg} /$ I Kinetin $+5 \mathrm{mg} /$ I BAP. The plant material consists of botanical seeds (True Potato Seeds-TPS) of two varieties registered under serial numbers (CIPN ${ }^{\circ}$ ) 997003 and 998010 . Induction of microtuberization was completed after 9 weeks of culture under dark conditions at $22^{\circ} \mathrm{C}$. for both varieties, the best rates of tuberization were obtained on MS3 medium. The increase in the concentration of BAP compared to kinetin causes a decrease in the rate of tuberization. However, the larger microtubers are produced on the MS1 medium for both varieties. The addition of Cytokinins in the microtubercule production media promotes the germination of microtubers. Keywords: Solanum tuberosum L, microtuber- cules, BAP, Kinetin.

P1 12. Water resources vulnerability factors in a global environmental change context: A systematic review and meta-analysis in international cases

Soumaila Kanga ${ }^{*}$, Mustapha Naimi, and Mohamed Chikhaoui

Soil and Water Management Laboratory, Department of Natural Resources and Environment, Hassan II Institute of Agronomy \& Veterinary Medicine, PO. Box 6202 - Madinat Al Irfane, Rabat, Morocco. *Corresponding and Presenting Author: soumkanga@gmail. com.

\section{Abstract}

Assessing the vulnerability of water resources is increasingly becoming an essential practice in the integrated water resources management. Accordingly, several studies have been carried out around the world during the last two decades. Thus, the main objective of this paper was to review factors used internationally to assess the vulnerability of water resources at local scale in order to adapt them and use them in the assessment of the vulnerability of water resources in a context of climate change in Morocco. To achieve this goal, we have pre-selected $187 \mathrm{pa}$ pers on water vulnerability in the Science Direct database of Elsevier publisher and the Google Scholar database. By applying the PRISMA protocol, only 13 articles out of the 187 selected met the inclusion or exclusion criteria previously defined for the analysis. The 113 water resources vulnerability factors identified were sorted out into five categories: physical or biophysical factors $(35.4 \%)$, socio-economic factors (31\%), environment or eco-environment factors (22.1 $\%)$, infrastructure factors (8.8\%) and water governance and institutions factors $(2.65 \%)$. Based on the availability of data, the scale of application and the environmental problems of water resources in Morocco, we were able to recognize 50 factors among all categories that could be used in the assessment of water resources vulnerability in Morocco. Keywords: Water vulnerability factors, integrated water management, water vulnerability assessment, local scale. 
$P 1$ 13. Evaluation of the Irrigation Water in the Central Bahira Plains of El Kalâa, Morocco

Soukaina El Hasini ${ }^{*}$, Mohammed El Azzouzi ${ }^{1}$, Houria Dakak ${ }^{2}$, Oumaima Ibnhalima ${ }^{2}$, and Abdelmajid Zouahri $^{2}$

1 Laboratory of Materials, Nanotechnologies and Environment, Faculty of Sciences, University Mohammed V, Av Ibn Battouta, BP1014, Rabat, Morocco; ${ }^{2}$ Research Unit on Environment and Conservation of Natural Resources, National Institute of Agricultural Research (INRA) PO Box 6356 Rabat-Institutes, Rabat, Morocco. "Corresponding and Presenting Author: elhasinisoukaina3@gmail.com.

\section{Abstract}

The aridity of the climate due to the succession of drought periods in morocco leads to a situation of water paucity. These Conditions make irrigation an unavoidable technical requirement for the development of agricultural lands. Even so, Agricultural intensification was accompanied by a lack of irrigation control which leads to several problems of degradation of water and soil quality in terms of salinity and alkalinity. This constitutes a serious danger to the sustainability of the land and water quality. In order to succeed in dealing with this situation as well as to ensure the sustainable preservation of these resources, the requirement of their monitoring and analysis becomes necessary. The present work falls within this framework suggests a synthesis of the hydrochemical situation by assessing the distribution of Salinity and other major problems affecting the quality of groundwater in the Bahira Plain, particularly around Sad Al Majnoun. An approach has been followed including a monitoring of the groundwater at the scale of the area according to a network of 33 water points. The measurements were carried out in situ $(\mathrm{CE}, \mathrm{pH}$, piezometric level) and in the laboratory for the major and secondary elements to specify (RSC, SAR and N\%). Moreover, the maps of the groundwater quality parameters have been established under GIS allowing us to delineate the areas affected by the problems of degradation of this resource as well as constitute a source of information and management for a best future monitoring of the qualitative state of the water table. Keywords: Irrigation water, hydrochemical situ- ation, cartography, GIS, central Bahira.

P114. Ability of non-pathogenic Fusarium oxysporum strain Fo47 to suppress Rhizomania disease of sugar beets in Morocco

Fatima Novayti ${ }^{1,2^{*}}$, Ilham Madani ${ }^{2}$, Abdessalem Tahiri ${ }^{1}$, Abdelali Blenzar ${ }^{2}$, and Rachid Lahlali ${ }^{1}$

1 Phytopathology Unit, Department of Plant Protection, Ecole Nationale d'Agriculture de Meknès, Meknès, Morocco; ${ }^{2}$ Moulay Ismail University, Faculty of Sciences. Department of Biology, P.O. Box 11201 , Meknes, Morocco. "Corresponding and Presenting Author: fatimanouayti@gmail.com.

\section{Abstract}

Rhizomania is one of the most devastating diseases of sugar beet worldwide. The disease poses a serious threat to Moroccan production and it is capable of significantly decreasing quality and yield of sugar beet plantations. The long-term survival of its fungal vector (Polymyxa betae) in soil makes it a very difficult disease to manage. Therefore, this study investigated the potential of a non-pathogenic fungal Fusarium oxysporum strain Fo47 to control Polymyxa betae. This biocontrol agent was applied as soil treatment, seed treatment, or a combination of the both treatments. A bio-test was performed on treated soil. After four weeks of culture, the roots of sugar beet seedlings were retrieved and analyzed by the DAS-ELISA test. Results indicated that F. oxysporium Fo47 reduced the activity and survival of $P$. betae when compared to a reference biocontrol agent Trichoderma harzianum, which only revealed significant in reducing the viral load of Beet Necrotic Yellow Vein Virus (BNYVV) as seed treatment. The non-pathogenic Fusarium oxysporum Fo47 was more effective as soil treatment and allowed almost the same reduction of BNYVV virus concentration as T. harzianum 908. Therefore, our findings emphasizes that the performance of the biocontrol agent depends on the method of application. Keywords: Beet Necrotic yellow vein Virus; Biocontrol, Fusarium oxysporum strain Fo47; Trichoderma harzianum strain 908. 
P1 15. Optimization of the technical management of agroforestry (choice of cereal species and nitrogen fertilization) in olive groves

Bendidi Abderrazzak ${ }^{1 *}$, Khalid Daovi', Rachid Dahani ${ }^{1}$, and Mohamed Ibriz ${ }^{2}$

${ }^{1}$ INRA, Meknes, Morocco; ${ }^{2}$ Faculty of Sciences, University lbn Tofail, Kenitra, Morocco. "Corresponding and Presenting Author: abendidi1@yahoo.fr.

\section{Abstract}

Intercropping is practiced on a large scale in Moroccan olive groves. According to the data from the feasibility study of the fruit growing project (PAF), cereal cultivation is practiced intercropped in more than $50 \%$ of the olive groves. In order to determine the influence of intercropped cereal farming techniques on olives trees, we studied olive tree production in association with two different cereal species (bread wheat and durum wheat) with two nitrogen doses (40 and 120 $\mathrm{kg} \mathrm{N}$ ha-1) in the Khenifra region. The results of the first year of study, characterized by drought and cold during the winter months, showed that durum wheat (Karim) allowed a grain yield (11.3 qx ha-1) higher than that obtained by bread wheat (Amal) ( $8.8 \mathrm{qx}$ ha-1). Regarding the nitrogen dose, the highest grain yield for wheat was recorded with $120 \mathrm{~kg} \mathrm{~N}$ ha- 1 with an average of $11.8 \mathrm{qx}$ ha-1, while grain yield with $40 \mathrm{~kg}$ qx ha- 1 was only 8.3 qx ha- 1 . While olive production of $45 \mathrm{~kg} /$ tree was not affected by either the cereal species or the amount of nitrogen added to the intercrop. These results show that the installation of cereals as an intercrop in Moroccan olive groves (under 16 years old for the Picholine marocaine variety) would allow the farmer to improve the productivity of his land and consequently the maximization of his income. Keywords: Intercropping, olive groves, bread wheat, durum wheat, nitrogen, production.

P1 16. Influence of Photovoltaic Panels on Growth and Productivity of Tomato Under Canarien Greenhouse

Kabira Ezzaeri $i^{1^{*}}$, Hicham Fatnassi ${ }^{2}$, Lahoucine Gourdo', Abderrahim Bazgaou', Ahmed Wifaya ${ }^{3}$, Rachid Bouharroud $^{3}$, Hassan Demrati', Ahmed Aharoune', Ahmed Bekkaovi ${ }^{4}$, and Lahcen Bouirden ${ }^{1}$
1 Thermodynamics and Energetic Laboratory, Faculty of Sciences, Agadir, Morocco; ${ }^{2}$ INRA, Univ. Nice Sophia Antipolis, CNRS, UMR 1355-7254 Institut Sophia Agrobiotech, 06900 Sophia Antipolis, France; ${ }^{3}$ Regional Centre of Agricultural Research, Agadir, Morocco; ${ }^{4}$ Mechanization Agricultural Department, IAV Hassan II, Rabat, Morocco. "Corresponding and Presenting Author: k.ezzaeri@uiz.ac.ma.

\section{Abstract}

Solar energy is one of the cleanest sources of alternative energy. Due to high energy demand in one hand, and environmental negative impact of fossil fuels on the other hand, many countries consider the alternative energy sources as a suitable and feasible option in industry and domestic usage. The use of renewable energy for greenhouse environment control to replace or reduce the consumption of fuel and powerline electricity is an important objective for sustainable greenhouse crop production. The photovoltaic greenhouse is a new technology for a "double use" of the resource land, because it generates energy in the form of biomass (crops, flowers, or algae) and, also, electric energy. The goal of our research is to study the development and yield of tomato inside a Canarian greenhouse, with $10 \%$ of their roof was occupied by photovoltaic panels. The results shows that the shading provides by the photovoltaic panels have not a significant effect on the tomato development and yield. Keywords: photovoltaic greenhouse, shading, tomato, yield.

P117. Epidemiology of multi-resistant bacteria in the hospital environment: service of major burns and plastic surgery, Mohamed V Hospital, Meknes

Naiia El hamzaoui ${ }^{*}$, Hassan Ouallal ${ }^{2}$, Abouddihai Barguigua $^{3}$, and Sbiti Mohammed ${ }^{4}$

1 Medical Microbiology Laboratory, Mohamed V Hospital, Moulay Ismail University, Meknes, Morocco; 2 Membrane Materials and Separation Processes Laboratory, Faculty of Science and Technics, Irrachidia, Morocco; ${ }^{3}$ Multidisciplinary Research and Development Laboratory, Department of Biology-Geology, Polydisciplinary Faculty, Beni Mellal, Morocco; ${ }^{4}$ Medical Biology Laboratory of Regional Military Hospital Moulay Ismail Meknes morocco. "Corresponding and 
Presenting Author: najia.elhamzaoui@gmail.com.

\section{Abstract}

Following several cases of bacterial infections, in the service of major burns and plastic surgery of Mohamed $V$ hospital, Meknes, samples were taken from this service to identify the bacteria responsible for these infections. Environmental sampling was carried out by the swabbing method. The cultures were realized for 48 hours at $37{ }^{\circ} \mathrm{C}$ on lactose agar, CLED, Mannitol Salt Agar (Chapman), blood agar and chocolate agar. The identification of the isolated bacterial species was carried out using the API gallery systems, the resistance level of the bacteria to the antibiotics was studied by the diffusion method in agar. the choice of antibiotics as well as the interpretation of susceptibilitytesting were performed according to CA-SFM recommendations. The surfaces and medical devices containing bacteria are of type: beds, surgical equipments,operating tables, bedside tables, door cuffs, stethoscopes, cupboards and washbasins. The identifiedbacteria are staphylococcus epidermidis and saprophiticus, Klebsiella penumoniae, Pseudomonas putida and acinetobacter baumanii, and the results of the antibiogram showed a predominance of multiresistant bacteria, especially Acinetobacter baumanii and Klebsiella penumoniae. Many observations have suggested that the contamination often resulted from inappropriate application of cleaning procedures, and many factors that are related to microorganisms:lifetime on a support, surface adhesion, ability to produce a biofilm and its ability to withstand adverseconditions. Considering the obtained results, the monitoring and the behavior study of bacteria, present in the service of the major burns, with regard to the more commonly used disinfectantsbecomes a necessity for the establishment of the appropriate disinfection procedures.

\section{P1 18. Long Term Monoculture Promotes Selection of Antagonistic Phonotypes Among Streptomyces in Soil.}

Essarioui Adil ${ }^{l^{*}}$ and Kinkel Linda $\mathrm{L}^{2}$

1 National Institute of Agronomic Research, Regional Center of Errachidia, Morocco; ${ }^{2}$ Department of Plant Pathology, University of Minnesota, USA. "Corresponding and Presenting Author: essar002@umn. edu.

\section{Abstract}

Streptomyces possess strong antagonistic activities and inhibit diverse plant pathogens. However, the effects of plant community characteristics on Streptomyces inhibitory activity have received little attention. We evaluated the impact of plant species and plant community richness on the frequency and intensity of inhibitory activity of rhizosphere Streptomyces communities against 2 fungal plant pathogens, Fusarium oxysporum f. sp. albedinis and Fusarium oxyxporum f. sp. lycopersici, the agents of date palm tomato wilts, respectively. Soil samples were taken from the rhizosphere of two plant species, Andropogan geradii and Lespedeza capitata, each growing in long term communities of 1 (monoculture) or 16 (polyculture) plant species. Streptomyces inhibitory activity against the two pathogens was studied using an in vitro assay to reveal inhibition on each target pathogen. For each soil sample, the proportions of Streptomyces that exhibit antagonistic activities against each pathogen and the mean size of the inhibition zone were determined. Plant richness modified the impacts of both plant species on Streptomyces antagonistic activity. Regardless of plant species, monocultures supported higher frequencies and greater intensities of Streptomyces inhibition than polycultures. Streptomyces antagonistic capacity increased with decreasing plant community richness, suggesting that plant diversity modifies selection for antagonistic phenotypes among soil Streptomyces. Fostering monoculture conditions in agricultural settings may contribute significantly to reducing the impacts of soil-dwelling crop pathogens. Keywords: Streptomyces, Inhibition, Monoculture.

\section{P1 19. Evaluation of Phosphate Solubilizing Bacteria Iso- lated from Cytisus monspessulanus Rhizosphere for their Growth Promoting and Antagonistic Activity Against Phytopathogenic Fungus Fusarium oxysporum}

Imane Achkouk ${ }^{1 *}$, SaidaAarab ${ }^{2}$, Amin Laglaoui', Mohammed Bakkali ${ }^{1}$, Abdelhay Arakrak ${ }^{1}$

${ }^{1}$ Equipe de Recherche de Biotechnologies et Génie des Biomolécules (ERBGB), Faculté des Sciences et Techniques de Tanger B. P. 416, Tanger, Morocco; ${ }^{2}$ Faculté des Sciences et Techniques d'Al Hoceima, B.P. 
34, Ajdir Al Hoceima, Morocco. 'Corresponding and Presenting Author: imaneachkouk@gmail.com.

\section{Abstract}

Plant growth promoting rhizobacteria (PGPR) are considered to be the most promising agents for agricultural crop via increasing crop yields and decreasing disease occurrence. The aim of this work is to evaluate phosphate solubilizing bacteria (PSB) isolated from the rhizospheric soil of Cytisus monspessulanus, for multiple plant growth promotion traits and to test their antagonistic effect against Fusarium oxysporum. A total of 37 isolates (15.81\%) among 234 isolated rhizobacteria were able to solubilize the tricalcium phosphate (TCP) on PVK solid medium. The 21 isolates with solubilization diameters $\geq 0.4 \mathrm{~cm}$ were selected and evaluated for the production of hydrogen cyanide, and the results showed that $52.38 \%$ of these isolates ( 11 isolates) produced this compound, so theywere retained for further tests. The amount of IAA produced during the quantitative test fluctuated between $55.45 \pm 9.52 \mathrm{mg} / \mathrm{L}$ and $2.35 \pm 0.38 \mathrm{mg} / \mathrm{L}$ and all the strains were positive for the production of sidérophores except CMR67.The selected rhizobacteria were then evaluated for their ability to secrete enzymes such as cellulase, chitinase and protease. The percentages obtained were respectively $81.81 \%$, $45.45 \%$ and $63.63 \%$. In addition, the ability of these PSB to produce ammonia was tested, and 9 strains $(18.18 \%)$ were able to produce this compound. All test strains were assessed by TCP solubilizing quantitative assay in PVK liquid medium and the concentrations of solubilized $P$ were between $147.49 \pm 0.06$ $\mathrm{mg} / \mathrm{l}$ and $7.57 \pm 0.04 \mathrm{mg} / \mathrm{l}$. This solubilization was accompanied by a $\mathrm{pH}$ decrease of the medium from 7 to 4.18 . The antagonism test against Fusarium oxysporum showed that all the selected isolates were able to inhibit its growth except the isolates CMP55 and CMR22. The best performance on all qualitative and quantitative tests was recorded by the three isolates CMR64, CMR68 and CMR78 that can be used as bioinoculants to contribute to the rehabilitation of degraded marginal soils. Keywords: PSB, PGPR, Cytisus monspessulanus, Fusarium oxysporum.

\section{P1 20. Ethnobotanical uses of Arbutus (Arbutus unedo} L.) in Morocco

Faida Rahima ${ }^{1,2^{*}}$, Aabdousse J. ${ }^{1}$, Boulli A. ${ }^{\text {, }}$, Bouda S. ${ }^{2}$, and Wahid N. ${ }^{1 \S}$

1 Ecology and Sustainable Development Team (ESDT), Department of Life Sciences, Faculty of Science and Technology, Sultan Moulay Slimane University, Beni Mellal, Morocco.

2 Laboratory of Management and Valorization of Natural Resources, Department of Life Sciences, Faculty of Science and Technology, Sultan Moulay Slimane University, Beni-Mellal, Morocco. "Presenting author: faida.rahima@gmail.com. \$Corresponding author: wahid2na@hotmail.com.

\section{Abstract}

Arbutus is a fruit tree with high ornamental, environmental, economic and medical value, because of the properties attributed to different biological parts. Given this interest, the present study is interested in describing and compiling available data on the ethnobotanical uses of Arbutus in Morocco in comparison with other studies at the international level. It is very present in the everyday uses of foresters, farmers and rural populations. The international bibliographic analysis shows that the different biological parts of Arbutus, especially fruits, have been widely used by herbalists. The related literature is generally in line with the medicinal use of the plant by Moroccan inhabitants. On the other hand, the consumption and the industrial valorization of the fruits of Arbutus are very limited in Morocco in comparison with those on the international scale. It is imperative to sensitize the local population and landlocked Douars on the knowledge of the valorization of the fruit of this species. Keys Words: Arbutus unedo L., ethnobotanical uses, prospection, ecology, Arbutus fruit.

\section{P121. Livestock genetic resources support resilience to} climate change in oasis production systems: A review

\section{Mustapha lbnelbachyr ${ }^{*}$}

National Institute for Agricultural Research, Center of Errachidia, PO Box 529, Boutalamine, Errachidia, Morocco. "Corresponding and Presenting Author: $\mathrm{m}_{\text {_ }}$ 
ibnelbachyr@yahoo.fr.

\section{Abstract}

Livestock, an essential component of production systems in oasis areas, is facing increasingly the effects of climate change. Livestock genetic resources play a significant role in promoting biodiversity and improving resilience to this phenomenon in oasis production systems. This review addresses the current status of preservation of animal genetic resources in the oases of southeastern Morocco, and presents the opportunities offered by these resources to meet future needs for oasis production systems. The focus is put on the typical oasis breeds; the Tidili cow, the D'man sheep, and the Drâa goat. The Tidili cow is typical cattle of the valley located between the southern slopes of the High Atlas and the Siroua massif. The main assets of this local breed include its perfect adaptation to local environmental conditions and its interesting milk production. The D'man sheep is the most prolific Moroccan sheep that has contributed to the improvement of meat production in the oases and in the crossbreeding areas. The Drâa goat is the goat breed of the oases of the DrâaValley which furrows the Moroccan southeast. It is the only Moroccan goat ableto get conceive at any period of the year and giving an important milk production. Thus, it constitutes a resource of genetic and economic interest certain. Overall, livestock breeds are genetically and economically important to improving resilience to climate change in oasis production systems. Preservation and genetic improvement programs are needed to prevent extinction of these breeds in the future. Keywords: Climate change, resilience, local breeds, oasis.

\section{P1 22. Protein, Oil, and Fatty Acids Contents of Soybean Mutant FM6-847 Grown in Fayetteville, North Carolina}

Safiah Kassem ${ }^{1 *}$, Jiazheng Yuan ${ }^{2}$, Naoufal Lakhssas$\mathrm{si}^{3}$, Khalid Meksem ${ }^{3}$, and My Abdelmajid Kassem ${ }^{2 \S}$

1 Pine Forest Middle School, 6901 Ramsey St, Fayetteville, NC, USA; ${ }^{2}$ Plant Genomics and Biotechnology Lab, Department of Biological Sciences, Fayetteville State University, Fayetteville, NC, USA; ${ }^{3}$ Department of Plant, Soil, and Agricultural Sciences, Southern Illinois University, Carbondale, IL 62901, USA. "Presenting author: safiahkassem@gmail.com. ${ }^{\S}$ Correspond- ing author: mkassem@uncfsu.edu.

\section{Abstract}

Proteins, oil, and fatty acids are important components of soybean [Glycine max (L.) Merr. ] seed composition. The objective of this study was to compare protein, oil, and fatty acids contents of the soybean mutant FM6-847 with three USDA reference lines (LD00-2817, LD06-7620, and LD07-3395) in a field in Fayetteville, NC in 2016. Our results showed that the mutant FM6-847 had significantly higher levels of protein than LD07-3395 but not LD00-2817 and LD06-7620. The mutant FM6-847 had significantly higher oil and palmitic acid contents than all three USDA reference lines. The mutant FM6-847 had a significantly higher stearic acid content than LDOO2817 and LD07-3395 but lower than the content in LD06-7620. For oleic and linoleic fatty acids, the mutant FM6-847 had significantly lower contents compared to all three USDA reference lines. The mutant FM6-847 had significantly higher linolenic acid content than LDO0-2817 but significantly lower levels than LD06-7620 and LD07-3395. The mutant FM6847 outperformed the USDA reference lines in yield, oil and palmitic acid contents; therefore, it is well adapted to North Carolina, is stable, and will benefit farmers in NC to increase their soybean yield, seed composition, and profit. Key words: Soybean, protein, oil, fatty acids, palmitic acid, stearic acid, oleic acid, linoleic acid, linolenic acid.

\section{P123. Response of two varieties of banana (Musa acu-} minata) small dwarf and large dwarf in vitro to salt stress

Meriem Belfakih ${ }^{1 *}$, Mohammed Ibriz ${ }^{1}$, and Abdelmjid Zouahri $^{2}$

' Laboratory of Genetics Biometry, Faculty of Sciences, University Ibn Tofail, BP 133, Kenitra, Morocco; ${ }^{2}$ Unité de Recherche "I Environnement et Conservation des Ressources NaturellesII, INRA, CRRA de Rabat, BP 6356 Rabat Instituts, Morocco. "Corresponding and Presenting Author: m_belfakih2000@yahoo.fr.

\section{Abstract}

The objective of this study is to evaluate the effect 
of salt stress $(\mathrm{NaCl})$ on the in vitro regeneration of banana and the possibility of using this technique to improve the tolerance of this species to salinity. Previously, the rejects were disinfected with $8 \%$ bleach and then grown in Murashige and Skoog medium (1962) containing growth hormones (Appendix 1). The medium was fed with different salt concentrations $(0,2,4$ and $6 \mathrm{~g} / \mathrm{I}$ of $\mathrm{NaCl})$. The test was conducted at a temperature of 28 to $30^{\circ} \mathrm{C}$ with artificial illumination and a photoperiod of $16 \mathrm{~h}$. The results obtained show that both varieties have regeneration of shoots despite saline treatment. However, salt affects leaf length of the explants and the regeneration rate. So, the increase in saline concentration induce to a decrease in the number of shootings per bud. the small dwarf variety appears to be sensitive to the salinity effect of the medium at all NaClconcentrations at the regeneration stage and the seedling stage. Keywords: Salt stress, regeneration, growth, in vitro, banana.

\section{P1 24. Impact of the combination of salt stress and min- eral nutrition on the growth of alfalfa biomass (Medi- cago sativa $L$ ).}

Meriem Belfakih ${ }^{1 *}$, Mohammed Ibriz ${ }^{1}$, and Abdelmiid Zouahri $^{2}$

1 Laboratory of Genetics Biometry, Faculty of Sciences, University Ibn Tofail, BP 133, Kenitra, Morocco; ${ }^{2}$ Unité de Recherche II Environnement et Conservation des Ressources Naturelles II, INRA, CRRA de Rabat, BP 6356 Rabat Instituts, Morocco. * Corresponding and Presenting Author: m_belfakih2000@yahoo.fr.

\section{Abstract}

This work aims to study the combined salt stress effects $(0.6$ and $12 \mathrm{~g} / \mathrm{I} \mathrm{NaCl})$ and the conditions of normal $(H N)$ and limiting (HD) mineral nutrition, on growth and mineral nutrition of three varieties of alfalfa (Sirever, Demnate 203 and Riche). The study was done under controlled conditions in a growth chamber for one month. The results obtained in this test showed that the salt treatment leads to a net reduction of the aboveground and root biomass upon addition of $6 \mathrm{~g} / \mathrm{l}$. This detrimental effect of salinity becomes less under normal supply conditions, with the exception of the 'Demnate 203' variety which showed the high- est yield under limiting supply conditions. Also, saline treatment leads to an accumulation of $\mathrm{Na}+$ cation and a reduction in $\mathrm{K}+$ absorption. The 'Siriver' variety was more sodium-inclusive under normal mineral supply (HN) conditions. In addition, the 'Demnate 203' ecotype produced more dry matter under salt conditions and limiting mineral supply (HD). Keywords: Alfalfa, Medicago sativa, Salinity, mineral nutrition.

\section{$P 125$. Evaluation of diverse sets of barley germplasm for resistance to scald disease (Rhynchosporium seca- lis)}

Houda Hiddar ${ }^{1,2^{*}}$, Sajid Rehman², Zakaria Kehel ${ }^{2}$, Athanasios Tsivelikas, ${ }^{2}$ Ramesh P.S. Verma ${ }^{2}$, Abdelkarim Filali-Maltouf ${ }^{1}$, and Ahmed $\mathrm{Amri}^{2}$

1 Microbiology and Molecular Biology Laboratory, Faculty of Sciences, Mohammed V University, Rabat, Morocco; ${ }^{2}$ Biodiversity and Integrated Gene Management Program (BIGM), ICARDA, Rabat, Morocco. *Corresponding and Presenting Author: hiddar.houda@gmail.com.

\section{Abstract}

Leaf Blotch, also known as scald, is one of the most destructive diseases of barley (Hordeum vulgare L.); caused by the haploid imperfect fungus Rhynchosporium secalis. Scald is widely distributed throughout the world and can cause $40 \%$ reduction in grain yield. The use of resistant cultivars remains to be the most effective, economical and environmental friendly way to control the disease but the genetic resistance to Moroccan isolates is poorly understood and only few sources of resistance have been identified. This study aims at the identification of new and effective sources of resistance to scald within various subsets: Best bet subset selected using the Focused Identification of Germplasm Strategy (FIGS; 80 accessions), reference set identified within the Generation Challenge Program (GCP; 204 accessions), and an association mapping panel (284 accessions) developed by ICARDA breeders. . All the accessions were sown and evaluated at seedling stage under controlled conditions. We have found that of the 568 barley accessions tested, 78 barley accessions (14\%) were found to be resistant, 86 accessions ( $15 \%$ ) to be moderately resistant, 104 accessions (18\%) to be susceptible, 
and 103 accessions (18\%) to be highly susceptible, respectively. Most of the resistant accessions (125; $22 \%$ ) showed immune reaction. These new sources of resistance will be a useful resource for resistance barley breeding programs around the world. Keywords: Barley, Scald, Rhynchosporium secalis, Resistance.

\section{P126. Analysis of the hydro-sedimentary process of a watershed in Western Rif of Morocco using the SWAT model: case of the Tleta watershed}

Fatiha Choukri ${ }^{1 *}$, Mohamed Chikhaoui', Mustapha Naimi' ${ }^{1}$ Damien Raclot ${ }^{2}$, and Yannick Pepin ${ }^{2}$

Département des Ressources Naturelles et Environnement, Institut Agronomique et Vétérinaire Hassan II, Rabat, Morocco; ${ }^{2}$ UMR LISAH, Institut de Recherche pour le Développement, Rabat, Morocco. "Corresponding and Presenting Author: choukri.fatiha@gmail.com.

\section{Abstract}

SWAT Model (Soil and Water Assessment Tool) is a tool for simulating flows in the landscape, useful for guiding management and groundworks within catchment areas. Accordingly, this model was tested to reproduce, on a daily timespan, water and sediment flows arriving to Ibn Batouta reservoir over the period 1980-2010. Various climatic (precipitation and temperature), morphologic (MNT) and thematic data (land use and pedology) were used for feeding the model. The measurements acquired at Ibn Batouta reservoir during the periods 1980-1982, 1983-1996 and 1997-2010 were used respectively for initialization, calibration and validation after running the sensitivity analysis to determine the most significant parameters. The performances indices of $R^{2}$, NSE and PBIAS were adopted to assess and control the quality of predictions of the monthly flows of water and sediments. The performance of prediction of monthly water flows is very high with $\mathrm{R}^{2}(0.95 ; 0.90)$, NSE $(0.94$; $0.89)$ and PBIAS $(-6 ; 2)$ respectively for both periods of calibration and validation. Meanwhile the performance of predictions of the monthly solid transport is very satisfactory with $\mathrm{R}^{2}(0.83 ; 0.64)$, NSE $(0.82$; $0.61)$ and PBIAS $(17 ;-6)$ respectively. Finally, SWAT model allows to properly reproduce monthly water and sediment flows within the Tleta catchment area and testing scenarios of management and groundworks to be considered. Keywords: SWAT, hydrological modeling, water erosion, Western Rif, Morocco.

\section{P127. Performance Analysis of a Modified Solar Still Equipped with Solar Tracker}

D. O. Maliani" ${ }^{*}$, A. Bekkaoui, E. H Baali, K. Guissi, Y. El Fellah, and H. Krim

Département Energie et Agroéquipements. Institut Agronomique et Vétérinaire Hassan II, Rabat, Morocco. "Corresponding and Presenting Author: drissi. oussama@gmail.com.

\section{Abstract}

Conventional solar still suffer from its low yield despite many attempts to optimize it. This is due to its high dependence of the low energy generated by the greenhouse effect. Hence, the concentration seems to be an effective way to increase heat energy received by the still, and consequently enhance its productivity. However, many researches addressed in the field do not benefit from this powerful technique with an efficient way. They usually use an intermediate item to transport heat to the water inside the still, such pipes filled by a heat transfer fluid, which causes a loss of energy and decreases the thermal performances of the solar still. The new idea, presented in this work deals with the design of an innovative system of solar distillation that concentrate directly the sun's rays on the water to be distilled while ensuring efficiency in a follow-up of the sun as well in the azimuth as elevation. The greenhouse effect will be used as an extra thermal energy. The aim was carried out to estimate the dynamic and thermal behavior of the concepts. An Arduino electronic card will ensure the control of the system. The yield, economic aspects and payback period of different solar still are examined, and possible conclusions with integration of dissimilar aspects are reached. Keywords: Solar still, Desalination, Solar tracker, Single slope.

\section{P1 28. Effect of temperature on date-palm syrup (Dibs) processing and quality}

Noufia Younés ${ }^{1 *}$, Outaleb Zahra ${ }^{2}$, Alem Chakib ${ }^{3}$, and Filali Zegzouti Younés ${ }^{4}$ 
1 National Institute of Agronomic Research, Errachidia, Morocco; ${ }^{2}$ Université Sidi Mohammed Ben Abdellah Faculté des Sciences et Techniques, Fès, Morocco; ${ }^{3}$ Research Team in Biochemistry \& Natural Substances, FSTE-UMI, Errachidia, Morocco; 4 Research Team in Biology Environment and Health, FSTE-UMI, Errachidia, Morocco. "Corresponding and Presenting Author: noutfiaa@yahoo.fr.

\section{Abstract}

In 2017, Morocco produced about 112,000 tons of dates. This production is characterized by a high percentage of KHALTS (clones / varieties with low quality). These date palm clones are sold to non- competitive prices or used for processing of others date-palm products. This investigation aims to contribute to the study and characterization of date-palm syrup as a by-product of this fruit. Thus, five samples dates of about $1500 \mathrm{~g}$ (about $1000 \mathrm{~g}$ coreless) each were used to manufacture the syrup at two extraction temperature $\left(70^{\circ} \mathrm{C}\right.$ and $\left.90^{\circ} \mathrm{C}\right)$. The main processing steps are: Coring, juice extraction, filtration and concentration of the juice. The obtained results of date samples are: Solids $=85.44 \pm 3.53, \mathrm{pH}=5.08 \pm 0.71$, Acidity $=0.15 \pm 0.048$ and Ash $=1.86 \pm 0.49$. The physical and chemical composition of syrup was: Brix $=74.03 \pm 0.95$, Solids $=82.00 \pm 1.42, \mathrm{pH}=$ $4.74 \pm 0.15$ and Ash $=1.62 \pm 0.10$. The yield production was about $64 \%$. Also, this study showed that the extraction temperature affects with a very highly significant manner $(\alpha=0.00018)$, the duration of juice concentration. This duration was being $9 \mathrm{~h}$ and $6.4 \mathrm{~h}$ for respective extraction temperatures of $90^{\circ} \mathrm{C}$ and $70^{\circ} \mathrm{C}$. However, the extraction temperature does not seem to affect yield, $\mathrm{pH}$ and dry matter of the syrup. Keywords: syrup, Dibs, date-palm fruit, temperature, quality.

P129. Mycorhization effect on Cork oak acorns and their Eco physiological behavior (Quercus suber L.) in drought conditions

Btissame Bouther ${ }^{1 *}$, Younes Abbas ${ }^{2}$, and Moustapha Arahou'

1 Botanical, mycology and environment lab, department of biology, faculty of sciences, university Med $V$ Rabat, Morocco; ${ }^{2}$ Faculty Polydisciplinary of Beni
Mellal, University Sultane Moulay Slimane, Beni Mellal, Morocco. "Corresponding and Presenting Author: bouther.btissame@gmail.com.

\section{Abstract}

The cork oak is one of the most important species in Morocco due to its socio-economic and environmental role. This species occupied a very large area whereas it decreases more and more these last decades, inspite of all the attempts of artificial regeneration which failed. In order to protect this species from extinction and subsequent strong deforestation, nursery cork seeds were planted in a substrate inoculated with three different ectomycorrhizal fungi under controlled conditions in order to evaluate the species: Effect on the development and resistance to drought of the cork oak. The plants inoculated by the fungus 3 showed very strong resistance which is explained by very low values in the height of the stem, the number of leaves and the stem diameter. Keywords: Cork oak, mycorhization, artificial regeneration, drought.

\section{P130. Efficacity of Ruta graveolens powder to fight against Callosobruchus maculatus (Coleoptera, Bruchi- nae)}

Lalla Fatima Douiri ${ }^{1^{*}}$, Nada Lafkih', Yasmine Ghoua$\mathrm{ti}^{2}$, Hayat Bouhareb', Oulghazi Said', Chakir Mery$\mathrm{em}^{3}$, Mgamat Fouzia $^{3}$, and Mohieddine Moumni ${ }^{1}$

1 Department of Biology Sciences Faculty, / Moulay Ismail University, P.B 11 201, Meknes, Morocco; ${ }^{2}$ Food Technology and Biochemistry Unit / National school of Agriculture, P.B. S/40 50000 Meknes, Morocco; 3 Department of Biology Sciences Faculty, / Ibn Tofail University, PB : 14000, Kenitra, Morocco. "Corresponding and Presenting Author: fatimadouiri@ yahoo.fr.

\section{Abstract}

Insects occur at every point along the chain of food production from the open field or greenhouse environment to larders and pantries in domestic premises. Their presence causes concern not only because of their appearance and the direct spoilage they cause to food products, but also because of the microbiological contaminants and pathogens they may 
carry, and because of possible allergenic reactions that their exuviae and frass may generate. This study focuses on the insecticidal activity of Ruta geaveolens powders against $C$. maculatus elevated at the expense of Cicer arietinum seeds (L). Laboratory's experiment was carried out under controlled conditions $\left(27^{\circ} \mathrm{C}, 70 \pm 5 \%\right.$ relative humidity and 12 hours of light). The results obtained for the studied parameters indicate that powders extrated from rosmary leaves and young twigs have an insecticidal activity against weevils, after ( 1 to 5 ) days exposure the lowest LC50 and LC99 range respectively from 87.58 to 13.19 and 157.34 to $26.52 \mathrm{mg} / 50$ graines for females and 52.63 to $15.90 \mathrm{mg} / 50 \mathrm{graines}$ and from 93.79 to $25.74 \mathrm{mg} / 50$ graines for males. In fact, longevity obtained with the control is 9.1 to 7.73 days, for males 5 to 3.7 days and for females de 6 à 4.7days. The Ruta powders exert negative effects on all the parameters of the weevil and can be used against $C$. maculatus. In addition, they are derived from botanical products, biodegradable, renewable source, economic and have low environmental impact and often undetectable. Keywords: Ruta geaveolens, Cicer arietinum, powders, Callosobruchus maculatus.

\section{P131. Improve the effeciency of gibberellic acid to in-} crease the fruit set and yield of seedless clementines mandarin of Morocco

Ismail Kaidi ${ }^{1,2^{*}}$, L. Messaoudi ${ }^{1}$, and Z. Messaoudi ${ }^{2}$

1 Département de Chimie, Université Moulay Ismail, Faculté des sciences, Morocco; 2 Département Arboriculture Fruitière Oléiculture Viticulture, Ecole Nationale d'Agriculture de Meknès, Morocco. "Corresponding and Presenting Author: ism.kaidi@gmail.com.

\section{Abstract}

The Sidi Aissa Clementine (Citrus reticulata Blanco) is a seedless mandarin of great fruit quality. It is the leading Clementine cultivar produced in Morocco. In recent years, Moroccan growers have planted thousands hectares of Sidi Aissa Clementine and more may be planted in the future. One of the major challenges to Moroccan growers is the problem of low fruit set and small fruit size associated with the production of seedless Sidi Aissa and other seedless Clementine mandarins. To overcome this problem, man- darin growers apply GA3 during bloom to enhance fruit set and yield. However, the current registration of gibberellic acid (GA3) for fruit set of Clementine mandarins in Morocco was based on experiments conducted in foreign countries and by private companies. In Spain and South Africa, GA3 is routinely used in the production of seedless mandarins to increase both fruit set and fruit size, but these strategies have not proven reliable in Morocco. The label provides no detailed information telling growers how to use GA3 to increase fruit set and yield or yield of commercially valuable large size fruit annually and, thus, results for Clementine mandarin production have been variable production have been variable. Moreover, improper use of GA3 results in low fruit set, canopy damage, and potential reduction of flower formation in following years. The goal of this research is to identify the proper dose and best timing of GA3 application for increasing fruit set and quality of commercially valuable fruit of Sidi Aissa Clementine mandarin and to determine the beneficts effects resulting from use of high rates of GA3 or from numerous applications of lower rates of GA3 the current and following years. On yield and fruit size in a comprehensive experiment testing the efficacy of combinations of GA3 concentrations and application times, the effect of which was unknown on mandarins, was undertaken to maximize the yield of quality valuable fruit of Sidi Aissa Clementine mandarin. Keywords: GA3, Clementine, Fruit set, Citrus flowers.

\section{P132. Status of Wilt and Root Rot (WRR) Disease of Kabuli Chickpea in Several Regions of Morocco}

Amine Elbouazaoui ${ }^{1,2^{*}}$, llyass Maafa ${ }^{2}$, Ahmed S. Kemal' $^{1}$, and Allal Douira ${ }^{1}$

${ }^{1}$ Ibn Tofail University Kenitra, Kenitra Morocco; ${ }^{2}$ International Center for Agricultural Research in the Dry Areas (ICARDA), Rabat, Morocco. "Corresponding and Presenting Author: amine.elbou10@gmail.com.

\section{Abstract}

Kabuli chickpea is the most important temperate food legume in wheat-based System of Morocco. The area covered by the crop is over 88,000 ha in $2015 / 16$ with a production of $44,000 t$ which is not enough to meet the demand of the population, a fact that pushed the country to imports chickpea to fill the gap. 
The low productivity is partly due to diseases and insect pests. Major chickpea diseases are Ascochyta blight and wilt/root rot (WRR) affecting spring and winter planted chickpea causing high yield losses. Although WRR disease is known to impact chickpea production, knowledge on disease incidence, distribution and pathogen associated with WRR complex are not well studied. For this purpose, a survey was conducted in two regions of Morocco (Gharb and Saiss) covering 19 fields, to determine the distribution and importance of WRR. The results showed that WRR incidence ranged from $1-30 \%$ where the majority of the field had 10 to $20 \%$ disease incidences. Laboratory isolation revealed that the major pathogens were Fusarium oxysporum f.sp. ciceris $(59.8 \%)$, Rhizoctonia bataticola $(32.7 \%)$ and R. solani $(7.3 \%)$ and very low incidence of Pythium spp. These results showed the need to develop cultivars resistant to Fusarium wilt and dry root rot. Keywords: Chickpea wilt root rot, Fusarium oxysporum fs ciceri, Morocco.

\section{P133. Induction of two Pathogenesis Related PR Pro- teins in Pear (pyrus communis) by Pathogenic and Non- Pathogenic Bacteria}

Naima Lemiiber ${ }^{\prime *}$, Abdelhai Dihazi', Nawal Zhar ${ }^{2}$, Hicham Jediyi ${ }^{2}$, Younes Rakibi², Wassila Arkiz ${ }^{2}$, and Khalid Naamani ${ }^{1}$

1 Laboratory of Biotechnology and Valorisation of Plant Resources, Faculty of Sciences Semlalia, Departement of Biology, Cadi Ayyad University, Marrakech, Morocco; ${ }^{2}$ Laboratory of Biotechnology for Valorisation and Protection of Agro-resources, Faculty of Sciences and Techniques Marrakech, Departement of Biology, Cadi Ayyad University, Marrakech, Morocco. "Corresponding and Presenting Author: naima. lemiiber2012@gmail.com.

\section{Abstract}

Plants represent a rich source of nutrients for many organisms including bacteria, fungi, protists, insects, and vertebrates. Although lacking an immune system comparable to animals, plants have developed a stunning array of structural, chemical, and protein-based defenses designed to detect invading organisms and stop them before they are able to cause extensive damage.The accumulation of pathogenesis-related proteins such as proteases and peroxidase was studied in two pear varieties, var.Williams and var.Louis Bonne inoculated by pathogen and non-pathogen bacteria. The bacillus pumilus strain increased also the activities of peroxidase and proteases after inoculation. The obtained results highlight the potential rol of bacillus pumilus in induction of defense-related enzymes. Keywords: Pathogenesis-related (PR) proteins, proteases, peroxidase, bacillus pumilus.

\section{P134. Antioxidant activity of Crataegus monogyna from Morocco}

Chaymae Benkirane ${ }^{1,2^{*}}$, Hakima Bahri ${ }^{1 \S}$, Bouchra Tazi $^{2}$, and Samira Oulahboub ${ }^{1,2}$

1 Laboratory of Genetic Resources and Plant Improvement, Ecole Nationale d'Agriculture de Meknès, , BPS 40, Meknès, Morocco; 2 Laboratory of Chemistry, Department of Basic Sciences, Ecole Nationale d'Agriculture de Meknès, BPS 40, Meknès, Morocco. "Presenting Author: chaymae.1.benkirane@gmail. com. \$Corresponding Author: hbahri@enameknes. ac.ma.

\section{Abstract}

Antioxidants are tremendously important substances which possess the ability to protect the body from damage caused by free radicals induced oxidative stress. The aim of this work was to investigate the antioxidant effect of hawthorn (Crataegus monogyna) collected from Ifrane region (Morocco) as a potential source of new bioactive natural compounds. Hawthorn is a medicinal plant widely used in phytotherapy for the treatment of many cardiovascular diseases. In this study, samples of flowers, leaves, ripened and unripe fruits were analyzed. The antioxidant activity was measured by DPPH (2,2-diphenyl1-picrylhydrazyl) free radical scavenging method. Then, Folin-Denis and aluminum chloride colorimetric assay were used to determine respectively the total polyphenol and the total flavonoid contents of Crataegus monogyna extracts. The results showed that all the plant parts possess important antioxidant properties. Unripe fruits and flowers revealed the highest antioxidant activity with IC50 values of 7.3 and $8.3 \mu \mathrm{g} / \mathrm{ml}$ respectively. Total polyphenol content in different plant parts ranged from 105.1 to 280.4 
GAE $\mu \mathrm{g} / 100 \mathrm{mg}$ extract and total favonoid from 4.7 to $70.8 \mathrm{QE} \mu \mathrm{g} / 100 \mathrm{mg}$ extract. Antioxidant activity shows a significant correlation with total polyphenol content. These results indicate that Crataegus monogyna exhibits a good antioxidant activity and thus can present a great potential as a dietary supplement or in pharmaceutical industry. Keywords: Crataegus monogyna, Antioxidant Activity, Total Polyphenol content, Total Flavonoid content.

\section{P135. Impact of Partial Root-Zone Drying and Wa- ter Deficit on Glucose, Protein, Polyphenols and Dry Matter Contents of Potato Grown Under Green- house Conditions}

Said Zantar 1*, Sliman Elhani 1, M. Haddadi1, and Mounir Hassani2

1 Nuclear techniques, Environment and Quality Research Unit. National Institute of Agronomic Research (INRA), 78 Bd. Sidi Med ben Abdellah 90010 Tangier, Morocco; ${ }^{2}$ Department of Biology, Polydisciplinary Faculty of Larache, University Abdelmalek Essaâdi. B.P 745, Larache, Morocco. "Corresponding and Presenting Author: zantar_said@hotmail.com.

\section{Abstract}

Water shortage is the most important factor constraining agricultural production all over the world. Potato (Solanum tuberosum L.) tuber yield and quality are impacted by irrigation management. The aim of this study was to evaluate the effects of two irrigation techniques on quality parameters of potato grown under glasshouse conditions. These techniques are deficit irrigation (DI) and partial root-zone drying (PRD). DI consists in irrigating the whole root system of the plant with an amount of water less than the crop evapotranspiration (ETc). PRD, in some way, is an evolution of $\mathrm{DI}$ in which the root system is divided between two containers to alternate wetting and drying of the root system. PRD and DI plants were irrigated according to transpiration demand for controls and the amount of water was reduced to $20 \%, 30 \%$ and $50 \%$ for both irrigation strategies. The following parameters were studied to evaluate the effect of PRD and $\mathrm{DI}$ on the quality: glucose, protein, polyphenol and dry matter content. Tubers from water stressed plants presented less contents of dry matter, glucose and proteins than the well-watered plants. Under PRD strategy, total polyphenols were reduced significantly only under severe water stress (50\% less than ETc). At the same irrigation level, PRD technique was more advantageous compared to DI in particular under moderate water restriction for total polyphenols, glucose and protein content. Keywords: Potatoes, deficit irrigation, quality, polyphenols, glucose, protein.

\section{P136. Estimation of Biochemical Propreties and Antioxi- dant Activity of Several Moroccan Plum (Prunus domes- tical.) Cultivars}

Youssef Ait Bella" ${ }^{*}$, Said Bouda, and Abdelmajid Haddioui ${ }^{\S}$

Laboratory of Biotechnologies and Valorisation of Plant Genetic Resources, Faculty of Sciences and Technics, Sultan Moulay Slimane University, P. B. 523, BeniMellal, Morocco. "Presenting author: youssef. gvrl@gmail.com. §Corresponding author: ahaddiovi@yahoo.fr.

\section{Abstract}

In this work, we report on the quality of 15 plum cultivars grown in different regions of Morocco. Plum (Prunus domestica L.) is among the most important stone fruit crop in the world. In Morocco, Prunus domestica L. is considered an important tree crops and is cultivated in different climatic regions. However, there is little information on the biochemistry of plums of the cultivars growing in Morocco. Fruits are analyzed by determining several parameters such as total polyphenols, flavonoids, antioxidant activity, total soluble solids, vitamin $\mathrm{C}$, titratable acidity, ash, humidity, dry matter, juice $\mathrm{pH}$, total sugars, fibers and crude proteins. The results showed that plum cultivars are characterized by a high nutritional value. In the other hand, the results showed significant differences between cultivars for the majority of the estimated parameters: total polyphenols, flavonoids, antioxidantactivity, total soluble solids, vitamin $\mathrm{C}$, titratable acidity, ash, humidity, dry matter,juice $\mathrm{pH}$, total sugars and fibers. Besides, the fruit mineral composition analysis (Zn, P, K, Mg, Ca...) revealed very high significant differences among the cultivars. The cultivars Lmozari and Tabarkakacht exhibited respectively the highest total polyphenols value $(9.39 \mathrm{mg} / \mathrm{g})$ and 
antioxidant activity $(70.65 \%)$ comparing with others introduced varieties. Therefore, some local cultivars with a particular chemotype could be recommended to cultivate at large scale. Keywords: Prunus domestica L., biochemical parameters, characterization, Cultivar.

\section{P137. Humic Substances Produced During Composting Process of the Olive Mill Wastes and Municipal Organic Wastes}

\section{Farid Barie ${ }^{*}$ and Mohamed Hafidi}

Laboratory of Ecology and Environment, Faculty of Science Semlalia, Cadi Ayyad University, Marrakesh, Morocco. "Corresponding and Presenting Author: fbarje@gmail.com.

\section{Abstract}

The humics substances form an important fraction of soils' organic compounds; their chemical nature has put forward various hypotheses on the mechanisms of their formation. In this context, to follow their evolution, the Humic acids(HAs) and Fulvic acids (FAs) extracted at different stages of co-composting of olive mill waste and organic household refuse with added phosphate, were analyzed with 13C-NMR and FT-IR spectroscopy. The findings highlight the contrasting variations in the proportions of C-aromatic and Ccarbonyl of $\mathrm{HAs}$ as the $\mathrm{H} / \mathrm{C}$ atomic ratio changes. The comparative 13C-NMR study of the FAs with the same tendancy, that show low aromatic $(C=C)$ content in the initial stage of composting, which contained a greater proportion of carbonyl $(C=O), O, N$-alkyl and alkyl carbon. The FT-IR spectroscopy of HAs showed a decreasing absorption in bands of aliphatic bonds $-\mathrm{CH}-,-\mathrm{CH} 2-$, carboxylic acids, esters, amides $\mathrm{C}=\mathrm{O}$, the C-O-bonds of polysaccharides and alcohols with respect to ether bonds $\mathrm{C}-\mathrm{O}-\mathrm{C}_{\text {; }}$ they was linearly correlated with the $\mathrm{H} / \mathrm{C}$ ratio. For the $\mathrm{FAs}$, the main $\mathrm{ab}$ sorption bands were in the region of $3393 \mathrm{~cm}-1$ (O$\mathrm{H}$ ), near $2931 \mathrm{~cm}-1$ (aliphatic C-H), a distinct shoulder between $1717 \mathrm{~cm}-1$ ( $\mathrm{C}=\mathrm{O}$ of carbonyl) and 1640 $\mathrm{cm}-1(\mathrm{C}=\mathrm{O}$ of amide and ketone), $1547 \mathrm{~cm}-1$ (aromatic $\mathrm{C}=\mathrm{C}$ with conjugated $\mathrm{C}=\mathrm{O}$ or amide $\mathrm{N}-\mathrm{H}$ ), near 1400-1384 cm-1 ( COO- stretch or OH deformation of $\mathrm{COOH}$ ). The ratio of HAs to FAs carbon content (CHA/CFA), which is representative of the polymer- ization degree, showed a positive linear correlation $(r=+0.56, p<0.05)$ with the degree of aromaticity of HAs. The increase in the CHA/CFA ratio with increasing humic acids aromaticity, is representative of a remarkable aspect of the restructuring brought, with respect to the mechanisms of polymerization during composting. Keywords: Humic Acids, Fulvic Acids, Composts, Organic wastes.

\section{P138. Mapping of Forest Fire in Morocco by Modis Products}

Issameddine Zidane ${ }^{1^{*}}$, Rachid Lhissou ${ }^{2}$, Abdelali Bou$\mathrm{li}^{1}$, and Mustapha Mabrouki ${ }^{3}$

1 Laboratoire d'Environnement et Valorization des Agro-Ressources, Department Science de la Vie, University Sultan Moulay Slimane, Beni Mellal, Morocco; ${ }^{2}$ Équipe de Recherche en Télédétection Environnementale et NORdique, Institut National de la Recherche Scientifique, Québec, Canada; ${ }^{3}$ Laboratoire de Genie Industrial, University Sultan Moulay Slimane, Beni Mellal, Morocco. "Corresponding and Presenting Author: z.issam.13@gmail.com.

\section{Abstract}

As in the Mediterranean countries, the forests in Morocco are exposed to a recurrent risk of fires due to the extreme flammability of forest species during the summer. Faced with this situation, the identification of burned areas and their monitoring are essential information to ensure the proper management and conservation of these ecosystems. This research focuses on the use of MODIS-Terra's medium spatial resolution satellite imagery in a semi-arid environment, particularly in the Rif region, which is in first place in terms of the number of fires and the area burned on a Moroccan scale. Our main objective is to map burned surfaces during the summer of 2016 using the two NASA forest fire mapping products derived from MODIS images: the MCD64A1 and MCD45A1. For this purpose, we carried out a field campaign to collect data on the presence of the fire and the measurement of the burnt surface combined with that of the HCEFLCD. The validation and comparison to show that MCD64A1 remains the most reliable in terms of fire presence, with a surface area greater than 25 ha and $R^{2}$ greater than 0.7. Keywords : Wildfire, Remote 
sensing, Burned area, MCD64A 1, MCD45A1, Modis.

\section{P139. Biochemical Characterization of Several Moroc- can Walnut (Juglans regia L.) Accessions}

Kabiri Ghizlane*-, Bouda Said, Elhansali Mohammed, and Haddioui Abdelmajid ${ }^{\S}$

Laboratory of Biotechnology and Valorization of Plant Genetic Resources, Faculty of Sciences and Technics, Sultan Moulay Slimane University, B.P. 523, Beni Mellal, Morocco. *Presenting Author: kghizlane84@ gmail.com. §Corresponding Author: ahaddioui@yahoo.fr.

\section{Abstract}

Juglans regia L. is a significant fruit in the nut species. Walnut has a high calorie level and rich nutrient composition. In Morocco the walnut is cultivated in various environments mainly on mountainous, as a traditional crop. The aim of this study is to investigate and to compare the biochemical parameters of 11 Moroccan walnut accessions. Walnut kernels are analyzed by determining several parameters such as the dry matter, the moisture, the total Ash, the total oil, the crude protein, the crude fiber, the carbohydrates, the energy value, the mineral composition $(\mathrm{P}, \mathrm{K}, \mathrm{Mg}, \mathrm{Na}$, $\mathrm{Cu}, \mathrm{Zn}, \mathrm{Mn}, \mathrm{Fe}, \mathrm{Cr}, \mathrm{Ni}, \mathrm{B})$, the phenolic compounds, the total flavonoid and the antioxidant activity. The obtained results show that Moroccan walnut is characterized by high nutritional value. On the other hand, the result showed significant differences between accessions for the majority of the estimated parameters, meaning of a high intra-diversity. This result should be exploited to select the best walnut accession. Keywords : Juglans regia. L - variability - biochemical - accessions.

P1 40. Evaluation of Some Nematophagous Fungi Isolates for their Biological Control Potential Against Rootknot Nematodes (RKN), Meloidogyne

Hafsa Tazi ${ }^{12 *}$, M. Ait Hamza', H. Boubaker ${ }^{2}$, and A. El Mousadik'

' Laboratoire Biotechnologie et Valorisation des Ressources Naturelles, Faculté des Sciences, Université lbn Zohr, BP 8106, Agadir, Morocco; ${ }^{2}$ Laboratoire Bio- technologie Microbienne et Protection des Végétaux, Faculté des Sciences, Université Ibn Zohr, BP 8106 , BP 8106, Agadir, Morocco. "Corresponding and Presenting Author: Hafssatazi@gmail.com.

\section{Abstract}

The Root-knot nematodes (RKN), Meloidogyne spp., are serious threat to many cultures around the world, particularly greenhouse crops. Nematicides are the most used method against plant parasitic nematodes. However, the majority of them were banned in developed countries because of their negative effects on both the environment and the human health. Hence looking for other ecological and effective management strategies has become a necessity. Biological control is considered the most relevant and least damaging approach as it is ecofriendly, economically viable and offers a sustainable and cost-effective alternative to chemical nematicides. The nematophagous fungi (NF) have been studied extensively as agents of biological control. This work aims the evaluation of nematicide activity of some NF strains isolated in nurseries. Strains were isolated, including a large diversity of Orbiliaceae with trapping organs and of toxic Hypocreaceae. Egg-parasitic and endoparasitic species were also observed. In vitro predation tests revealed that 7 isolates have increased the immobility of juveniles (J2).The most effective isolate was HT2 1 which increased the percentage of immobility up to $76 \%$ followed by HT1 and HT2 with $66 \%, \mathrm{HT} 30$ with $48 \%, \mathrm{HT} 13$ and $\mathrm{HT} 14 ; 29 \%, 20 \%$ respectively and finally HT22; $12 \%$. The other isolates have shown a percentage of immobility under $10 \%$. The active fungal isolates are candidates for studies on identification of potential nematicides. Hence the strains of fungi that have shown their efficacy against plantparasitic nematodes will be used as bionematicides. Keywords: Root-knot nematodes, Biological control, nematophagous fungi, bionematicides

P141. Analysis of Quality Parameters Related to the Maturity of Peaches and Nectarines in the Sais Region

lbtissame Guirrou ${ }^{1 *}$, A. Mamouni ${ }^{2}$, A. Mekaoui ${ }^{2}$, and K. Elfazazi ${ }^{3}$

1 Unité de Recherche Gestion des Ressources naturelles, Socio économie et Qualité, Qualipôle-Agropolis, 
INRA, Meknès, Morocco; ${ }^{2}$ Unité de Recherche Amélioration des plantes et Conservation des Ressources phyto-génétiques, Centre Régional de la Recherche Agronomique de Meknès, INRA, Morocco; ${ }^{3}$ Laboratoire de Recherche en Agroalimentaire, Centre Régional de la Recherche Agronomique de Tadla, INRA, Morocco. "Corresponding and Presenting Author: guirrouibtissame@yahoo.fr.

\section{Abstract}

As a perishable fruit, peach and nectarine can only be consumed fresh or transformed. So the choice of the date of harvest is very important because it directly influences the quality of the fruits, knowing that it is during the maturation that elaborates the organoleptic quality of the fruits having a strong impact on the market value: size, visual appearance, odor, and fruit preservation. Before doing the experimental work, we have done a survey in order to collect the information, concerning the variety range used and the distribution of the periods of maturity as well as the spread of the availability of the fruits on the market in the region of Sais. The diagnosis of the varietal range has shown the presence of a great diversity (more than 200 varieties). We have found a dominance of varieties: Patty, Gypsum, Azurite for peaches and Carolina, Turquoise, Emerald and Zephyre for nectarines. The fruit maturity is spread over 5 months, from early May to late September. Some experimental analysis has been done in order to focus on the identification of fruit quality parameters. The parameters studied are the measurement of size/ weight, firmness, color, moisture content, refractometric index (Brix), acidity, $\mathrm{pH}$ and total phenolic content. In order to cover the maximum, for each variety, we have practiced between 5 and 6 passages more than what is practiced by the farmer ( 3 passages) for 3 exploitations at Sais region. The results showed that early varieties have a smaller size, firmness and sugar content compared to seasonal and late varieties. While, late varieties have a relatively low acidity compared to early and seasonal varieties and therefore $\mathrm{pH}$ values $>4$ classify them as non-acidic fruits. Furthermore, it has been shown that the total phenolic compounds are not influenced by the season (early and late). These compounds are probably determined by the genetic potential of the variety. Indeed, nectarines have a slight wealth compared to peaches.
Keywords: peaches/nectarines, maturity, quality parameters, early/seasonal/late varieties.

\section{P142. In-vitro Synergic activity between essential oils and methanolic extracts of two medicinal plants from Algeria}

\section{Elkolli Hayet $^{1^{*}}$ and Elkolli Meriem ${ }^{2}$}

' Laboratoire des Matériaux Polymériques Multiphasiques, Département de Génie des Procédés, Faculté de Technologie, Université Ferhat Abbas, Setif 1, 19000, Algeria; ${ }^{2}$ Laboratoire de Valorisation des Resources Biologiques Naturelles, Faculté des Sciences de la Nature et de la Vie, Université Ferhat Abbas Setif 1, 19000, Algeria. * Corresponding and Presenting Author: Kolli_h@yahoo.fr.

\section{Abstract}

Due to the increased interest in alternative natural substances (essential oils and plant extracts) for their low toxicity nature, their combinations or mixings represented a potential new strategy for developing new anti-oxidant therapy. The use of combinations thus new approaches to increase their efficacy, taking advantage of their synergistic and additive effects. The purpose of this review is to provide an overview on the efficacy of these combinations. So we have investigated antioxidant activity of individual essential oils and methanolic extracts, then we have studied the antioxidant activity of their combinations. All the combinations gave a synergical effect. According to the IC50 parameter, the radical scavenging capacities are ranked in the following descending order: $B$. incrassatum EO $>$ B. alpinum EO $>$ EO mixing $>$ Quercetin $>$ Rutin $>$ BHT $>$ B. alpinum EXT $>$ EXT mixing $>$ B. incrassatum EXT. Keywords: Essential oil, methanolic extract, Bunium alpinum, Bunium incrassatum, combination, antioxidant activity, synergy.

P 143. Effect of a Sulla-based Diet (Hedysarum flexuosum) on the Productivity and Milk Quality of Local Beni Arouss Goat

Mohamed Ayadi ${ }^{\text {** }}$, A. Errassi ${ }^{2}$, M. Chabbi ${ }^{2}$, and S. Zantar $^{1}$

1 Laboratory of Materials and Valorization of Re- 


\section{Abstract}

Productivity, chemical composition and fatty acid profile of milk were studied in order to highlight the effect of the incorporation of Sulla (Hedysarum flexuosum) into the diet of Beni Arousse goats in comparaison with Alfalfa (Medicago sativa). 14 goats were divided into two homogeneous groups and milk collection was carried out weekly. Monitoring milk production during the first three months of lactation for each batch $(N=7)$ has shown that the use of sulla as main food has affected significantly and positively the productivity of Milk (+ 15\%). No significant variation was recorded for $\mathrm{pH}$ and acidity. On the other hand, the analysis of the chemical composition using Milkoscan revealed that the milk from the test batch is characterized by a slightly higher average content of lactose (5.23 vs $5.13 \%$ ) and defatted dry extract $(10.31$ vs $10.12 \%)$, while the protein and fat content were not significantly affected ( $P>0.05)$, knowing that the price of milk is estimated essentially from these two parameters. Analysis by gas chromatography showed that the incorporation of Sulla has beneficial effects on the fatty acid profile of the milk and which can be summarized essentially by a significant decrease of $10.51 \%$ for palmitic acid content (C16:0) and a significant increase of $28.99 \%$ in the family of omega-3 fatty acids which are considered as highly beneficial. In fact, these results show that the exploitation of Hedysarum flexuosum appears as a promising strategy for improving the quality of the local goat's milk. Keywords : goats, lactation, Sulla, chemical composition, fatty acids.

P1 44. Identification and Pomological Evaluation of Apple (Malus domestica Borkh.) cultivars in Morocco

Youssef Khachtib-, Said Bouda, and Abdelmajid Haddioui $^{\S}$

Laboratory of Biotechnologies and Valorization of Plant Genetic Resources, Faculty of Sciences and Techniques, Sultan Moulay Slimane University, P. B.

523, Beni Mellal, Morocco. "Presenting Author: yosf5achtib@gmail.com. §Corresponding Author: ahaddioui@yahoo.fr.

\section{Abstract}

Apple (Malus domestica Borkh) is the second most important tree fruit crop of the Rosaceae family in terms of cultivated area in Morocco, after almond tree; but global apple production involved only a few numbers of cultivars. Besides, the role of local cultivars and landraces in large-scale production has become completely insignificant in front of the introduced ones. In order to identify and evaluate the genetic resources of apple in Morocco, several prospecting were done and leading to collect 29 cultivars throughout its cropping area. 32 pomological traits were considered to analyze the phenotypic variability of the cultivars. The results of the analysis of variance showed very high significant differences between cultivars for almost all measured parameters, implying the existence of high phenotypic variability in Moroccan apple cultivars. Fruit shape, weight, length, and texture were revealed to be the most discriminating traits between analyzed cultivars. The autochthonous cultivars have shown the lowest values of considered characters compared with modern cultivars (introduced). This is clearly appeared with local Lahlou cultivar that obtained the lowest values of fruit weight, fruit height and fruit size. Nevertheless, the local and introduced apple cultivars were gathered in one group, according to principal components and hierarchical analyses, suggesting that they have had a common ancestor and a common genetic basis. Furthermore; some mislabeling of apple cultivars could be detected. The results of this study are discussed in the light of how this variation within traditional cultivars can serve as an interesting potential of biodiversity for future apple breeding and germplasm conservation programs. Keywords: Apple, local cultivars, introduced cultivar, Pomological, Variation.

P145. Biocide Effect of Ammi visnaga ( $L$ ) and Asphodelus cerasiferus Gay. Powder on Callosobruchus maculatus F. (Coleoptera, Bruchidae)

Fouzia Mgamat ${ }^{1 *}$, Ahmed Boughdad ${ }^{2}$, Fatima Doui$\mathrm{ri}^{3}$, Rachid Zohair ${ }^{3}$, El Ayadi Rachida', and Hamid El Ibaovi' 
1 Nutrition, Health and Environment laboratory, Departement of Biological Sciences, Faculty of Sciences, Ibn Toufail University, Kenitra, Morocco; ${ }^{2}$ Departement of Zoology, ENAM, Meknes, Morocco; ${ }^{3}$ Departement of Biological Sciences, Faculty of Sciences , My Ismail University, Meknes, Morocco. "Corresponding and Presenting Author: mg.fouzia@yahoo.fr.

\section{Abstract}

In the perspective of looking for alternative methods of fighting a pest of stored products, it is proposed to evaluate the potentialities of Ammi visnaga (L) and Asphodelus cerasiferus Gay. powder as botanical insecticides for the protection of chickpea seeds ( $\mathrm{Ci}$ cer arietinum) against Callosobruchus maculatus (Fab). Thus, 50 seeds of the chickpea were dusted separately and respectively with the powder of khella seeds and the roots of asphodel at the rate of 0 ; $0.16,0.32$, and $0.64 \mathrm{~g}$ for $\mathrm{A}$. visnaga, and $0,0.12$, 0.23 and $0.46 \mathrm{~g}$ for Asphodel. The treated seeds were then presented to 10 pairs of newly emerged C. maculatus. Three repetitions were done within each lot. The results showed that the powders of $A$. visnaga and $A$. cerasiferus did not affect neither the longevity, the fecundity nor the success rate of $C$. maculatus. Keywords : Cicer arietinum, Ammi visnaga, Asphodelus cerasiferus, Botanical insecticides, Stored products.

P1 46. Responses of Stomatal Conductance, Leaf Water Potential and Water-Use Efficiency in Potato to Partial Root-Zone Drying and Deficit Irrigation Under Glasshouse Conditions

\section{Sliman Elhani ${ }^{{ }^{*}}$, Said Zantar ${ }^{1}$, and Maroua Haddadi ${ }^{1}$}

1 Nuclear techniques, Environment and Quality Research Unit. National Institute of Agronomic Research (INRA), 78, Bd. Sidi Med. Ben Abdellah, Tangier, Morocco. "Corresponding and Presenting Author: sliman_ elhani@yahoo.fr.

\section{Abstract}

Partial root-zone drying (PRD) is a deficit irrigation (DI) technique which consists in dehydrating half of the root system whereas the other half is well irrigated, so that abscisic acid produced in the drying roots reduces stomatal conductance and limits tran- spiration. Aiming at comparing this technique to DI under glasshouse conditions, an experiment was conducted on potato (Solanum tuberosum L.) to evaluate stomatal conductance, leaf water potential ( $\Psi L)$ and water-use efficiency (WUE). PRD and DI plants were irrigated on transpiration demand determined by the weight of the pots. Treatments consisted of four levels of water supply for each irrigation strategy: $100 \%, 80 \%, 70 \%$ and $50 \%$ of crop evapotranspiration (ETc). Results showed no significant differences in tuber yield between irrigations strategies whereas statistically significant differences were found among treatments. PRD strategy reduced stomatal conductance and increased leaf water potential. WUE was higher under PRD but did not show statistically significant differences with DI. Using the same amount of water, PRD strategy allowed better yield and positive physiological responses to water restriction. Keywords: potato, partial root-zone drying, stomatal conductance, leaf water potential, WUE.

\section{P147. Agronomic and Molecular Characterization of a Collection of Faba Bean (Vicia faba L.)}

Chetto Oumaima ${ }^{*}$, Belqadi Loubna, and Arbaoui Mustapha

Production, Protection and Plant Biotechnology Department, Hassan II Institute of Agronomy and Veterinary Medicine, Rabat, Morocco. 'Corresponding and Presenting Author: oumaima.chetto@gmail.com.

\section{Abstract}

Despite the importance of faba bean (Vicia faba L.) in Morocco, its acreage has decreased due to a number of biotic and abiotic constraints but also due to the small number of varieties available. In order to contribute to the genetic improvement and the creation of new varieties, a study of phenotypic and molecular diversity was carried out on a large number of Moroccan and foreign accessions. This plant material was evaluated on the basis of nine agro-morphological traits in two locations; Taounate and Tiflet within the framework of Seminov's breeding program. In addition, the collection was analyzed using the molecular marker SRAP with six primers, in a laboratory of IAV Hassan II. The study of diversity revealed a high polymorphism with an extremely significant environmental 
effect as well as a genotype effect for the majority of agro-morphological traits. On the basis of these same features, distance analysis revealed that accessions of foreign origins are distant from Moroccan accessions and constitute an isolated cluster. The Mediterranean genotypes from Spain and Egypt, fit in among the Moroccan accessions. Furthermore, this study also contributes to develop the SRAP marker technique on agarose gel to evaluate genetic diversity. This characterization revealed an average polymorphism rate of $86.2 \%$. The analysis of distance also revealed a grouping which fits partially with the phenotypic analysis results. The association between the SRAP markers and the quantitative traits revealed the presence of some significant associations to more than $95 \%$ (tstudent): M3-E1-5, M3-E2-1, M3-E2-2, M1-E2-2 and M1-E2-4 markers seemed to be associated with the orobanche tolerance; the yield is associated with M3E1-4, M3-E2-6 and M2-E6-3 markers; and the TGW to M2-E6-3 and M3-E1-6 markers. Additional studies should be carried out on a larger number of genotypes to validate those molecular markers for their potential use in marker-assisted selection. Keywords: Vicia faba L., SRAP, genetic diversity, marker - trait association

\section{P148. Optimization of controlled pollination in Argan tree (Argania spinosa L.)}

Naima Ait Aabd ${ }^{*}$, Mohamed Boujghagh, Rachid Bouharroud, Abdelghani Tahiri, and Abdelaziz Mimouni

National Institute of Agronomic Research, CRRA Agadir, BP 124, Inezgane, Agadir, Morocco. "Corresponding and Presenting Author: aitaabdnaima@gmail. com.

\section{Abstract}

Pollination is a critical stage in sexual plant reproduction and is a key step to assure fertilization and seed production for many plants. The argan tree is a monoecious species, with hermaphrodite flowers, alone or grouped in glomeruli. In Fact, the pollination system of argan tree is similar to several angiosperms with more frequent insect pollination in natural conditions. However, few studies assess the quantitative field assessment of pollination modes and sexual breeding system of argan tree. In this work different steps have been optimized to assess self and cross-pollination experiments in argan tree in order to evaluate the reproductive biology and directed crosses in breeding programs. Hence, we report the sexual and breeding system of 23 trees in INRA orchard installed since 2010 in MZD, Agadir. The breeding system was determined by hand, self and cross-pollination experiments. Inflorescences were selected, bagged and the following treatments were assigned to each of the selected branch. Open/natural pollination (control), autogamy (isolated pollination), hand self-pollination and hand cross-pollination. The obtained results show strength of incompatibility within trees and $A$. spinosa is largely self-incompatible. The trees showed high heterozygosity in floral and seed development. Likewise, the obtained results are useful to optimize controlled pollinations in Argan tree and can help to increase fruit production in breeding programs and avoid the incorrect interpretation from pollination experiments in studies of reproductive biology. Keywords: Arganiaspinosa, hand pollination, cross-pollination, self-incompatible, seed production, breeding system.

\section{P149. Screening of Actinobacteria from Sugar Beet Soils in Beni Mellal Region for their Phosphate Solubiliz- ing Capacity}

Yassine Aallam ${ }^{*}$, Meriam Bousselham, Abdelmajid Haddioui, and Hanane Hamdali ${ }^{\S}$

Laboratory of Genetic and Plant Biotechnology, Faculty of Sciences and Technology, University of Sultan Moulay Slimane, B.P: 523 Beni Mellal, Morocco. "Presenting Author: yassine.aallam@gmail.com. §Corresponding author: hamdali_hanane@yahoo.fr.

\section{Abstract}

Agriculture will have to face the challenge of meeting the growing demand for food, which requires intensive application of chemical fertilizers like Phosphate which is the second most important nutrient for plant growth, but its availability is often reduced. Therefore, high quantities of expensive chemical soluble P-fertilizers are added to soil. Therefore, it is becoming a necessity today to develop a modern and sustainable agriculture with alternative methods that are less expensive, less polluting but equally effec- 
tive. Like the direct use of phosphate rock (PR) as an alternative to chemical P-fertilizers associated to phosphate rock-solubilizing actinobacteria (PRSA) in order to increase PR reactivity. Therefore, the main objective of this work was to obtain competent PRSA presenting other PGPR-associated traits to be used for the development of an inoculant to improve sugar beet growth and $P$ nutrition. In this study, four different sugar beet soils of Beni Mellal region (Morocco) were explored for the presence of phosphate solubilizing actinobacteria. 164 strains of actinobacteria were isolated and tested for their ability to solubilize a phosphate rock (PR). Among tested isolates, more than 34\% (57 strains) showed growth on synthetic minimum medium (SMM) containing insoluble PR and 16\% (27 strains) on SMM containing tricalcium phosphate (TCP) as unique phosphate source. Twenty isolates showed the most active growth and solubilization ability in the presence of PR and TCP in broth cultures. Thus, this work could contribute to select some actinobacteria as potential PRSA for use in low-input agriculture in low-P soils amended with PR. Keywords: Actinobacteria, Sugar Beet, soils, screening, phosphate rock solubilization, Beni Mellal.

\section{P150. Modeling and Simulation of Convective Solar Drying of Sewage Sludge}

Toky A. A. Arisily ${ }^{1 *}$, Said Hachimi ${ }^{2}$, Abdellatif Djerrari $^{1}$, and Ali Haiji'

1 Process Engineering and Environment Research Unit, Institut Agronomique et Vétérinaire Hassan II, Rabat, Morocco; ${ }^{2}$ Ecole Nationale Supérieure des Mines, University Mohamed V, Rabat, Morocco. "Corresponding and Presenting Author: tokyarisily@gmail.com.

\section{Abstract}

In recent years, solar sludge drying has attracted more and more communities, including in Morocco, due to its low operating costs and its respect for the environment. The objective of this work is to develop a lumped parameters dynamic model for convective solar drying of sludge from a waste water treatment plant (WWTP), then to simulate and validate the model with the experimental data and results found in the literature. A mathematical model of this process will make it possible to optimize the design and the exploitation of these driers, as well as the control of the characteristics of the sludge produced according to end-use or disposal chosen. First, based on the literature, a brief discussion is presented on some models, heat and mass transfer parameters, and sludge drying kinetics. Secondly, a lumped parameters dynamic model representing the different exchanges of heat and mass within the solar dryer is developed. The finite explicit difference method is used for solving the equations. These equations have been programmed in Excel VBA to form a simulation tool. Third, the experimental data and results of Amadou (2007) were used for simulations and validate the established model. A good agreement was found between the experimental results presented by the author and those given by the model. Keywords: Sewage sludge, solar drying, modeling, simulation, WWTP.

\section{P151. Genetic Diversity of Faba Bean (Vicia faba L.) Landraces in the Moroccan Rif Region using Molecular AFLP Markers}

Nora Aqtbouz ${ }^{1 *}$, Lamiae Ghaouti', Loubna Belqadi', and Wolfgang Link ${ }^{2}$

1 Département de Production, protection et Biotechnologies Végétales, Institut Agronomique et Vétérinaire Hassan II, B.P. 6202, Rabat-Institut, Rabat, Morocco; ${ }^{2}$ Institute of Agronomy and Plant Breeding, von Siebold Straße 8, 37075 Göttingen, Germany. ${ }^{*}$ Corresponding and Presenting Author: noraaqtbouz@ gmail.com.

\section{Abstract}

Faba bean (Vicia faba L.) is the most important grain legumes grown in Morocco. This crop is mainly produced based on locally maintained varieties and seeds (about $98 \%$ ), following an informal system of seed production and supply. These local varieties are characterized by a huge genetic diversity and are remarkably well adapted to the traditional agroecosystem and farming system. Assessment of genetic diversity is the first step of every plant breeding program. In fact, use of new methods for studying genetic diversity seems important. The objective of the present study was to describe the genetic diversity of a representative collection of faba 
bean local populations originating from four regions in the province of Taounate, using the Amplified Fragment Length Polymorphism (AFLP) molecular marker. Four AFLP primer combinations were used on 180 samples of faba bean local populations. A total of 139 amplified DNA fragments, varying in size from 60 to 380 base pairs, were revealed. From those, $84(60.43 \%)$ were polymorphic. The AMOVA analysis showed significant levels of variation among and within the four regions analyzed. A large extent of variation occurs within regions $(98.70 \%)$. The UPGMA dendrogram constructed based on the Jaccard's similarity coefficient indicated no clear specific pattern or grouping among these samples analyzed according to their geographical origin. All faba bean samples were displayed in one main cluster, divided in subgroups, which represent some degrees of similarity. Keywords: Faba bean, Local population, AFLP, Genetic diversity, UPGMA, Jaccard similarity coefficient.

P152. Occurrence of faba bean (Vicia fabae L.) diseases in five regions in Morocco

Krimi Bencheqroun Sanae ${ }^{1^{*}}$, Aouzal Sarra ${ }^{1,2}$, Lhaloui Saadia $^{1,3}$, and Seid Ahmed ${ }^{3}$

1 National Institute of Agricultural Research (INRA), Settat, Morocco; 2 Mohamed V university, FST- Settat, Morocco. 3 International Center for Agricultural Research in the Dry Areas (ICARDA), Rabat, Morocco. *Corresponding and Presenting Author: krimisanae@ gmail.com.

\section{Abstract}

Survey were conducted during three successive cropping seasons 2013, 2014, 2015 in five major Faba bean growing regions in Morocco (Abda, Doukkala, Saiss, Zemour zair and Chaovia). The aim of this study is to find out the occurrence and distribution of the principal cryptogamic diseases of faba bean affecting yield and quality of cropping. The results showed that Chocolate spot (Botrytis spp.) was the most widespread and destructive disease with high incidence in all surveyed regions. Ascochyta blight (Ascochyta fabae) and Rust (Uromyces fabae) were also important diseases mainly in humid regions (Saiss and Zemmour Zaer). In some fields, the late infection of Ascochyta blight in the season had affected yield and seed quality. Other diseases of minor importance were recorded at low incidence levels in some regions were Downy mildew (Peronospora viciae) and root rots. Therefore, screening for resistance to the major diseases observed in this study has to be intensified within faba bean breeding program in Morocco.

\section{P153. Solubilization of organic and inorganic phosphate by soil bacterial isolates}

Nabil Tirry ${ }^{1 *}$, G. Laghmari', B. Benbrik', R. Bouamri ${ }^{2}$, W. Bahafid' ${ }^{\text {, and N. El Ghachtouli }}{ }^{1}$

' Laboratoire de Biotechnologie microbienne, Faculty des Sciences and Techniques, Université Sidi Mohammed Ben Abdellah, Fès, Morocco; ${ }^{2}$ Département de protection des plantes et de l'environnement, Ecole Nationale d'Agriculture de Meknès, Morocco. "Corresponding and Presenting Author: nabil.tirry@usmba. ac.ma.

\section{Abstract}

Phosphorus $(\mathrm{P})$ is one of the major essential macronutrients for plant growth and development. However, $P$ in soil is generally present in two main insoluble forms: mineral forms, such as apatite, hydroxyapatite and oxyapatite, and organic forms including inositol phosphate, phosphomonoesters, phosphodiesters and phosphotriesters. Microorganisms are capable of converting insoluble phosphate into a bioavailable form through solubilization and mineralization processes. Hence, in the present study fifty-seven phosphate solubilizing bacteria were isolated from tree different agricultural sites in Morocco using NBRIP-agar containing tricalcium phosphate (TCP) as the phosphate source. The isolates were able to solubilize TCP in NBRIP liquid medium, with different capabilities (up to $190 \mu \mathrm{g} / \mathrm{ml}$ ). P solubilization was accompanied by a decrease in the $\mathrm{pH}$ of the medium. Crude alkaline and acid phosphatase activities of the strains were determined by p-nitrophenyl phosphate assay. $59 \%$ of the isolates showed alkaline phosphatase activity, $72 \%$ showed acid phosphatase activity, while $49 \%$ displayed both alkaline and acid phosphatases activities. The isolates were tested for other PGP traits. Results showed that $71.92 \%$ of the bacterial isolates are able to produce siderophores, $45.61 \%$ produce $\mathrm{HCN}$ and all isolates produce AIA (up to $500 \mu \mathrm{g} / \mathrm{mL}$ ). 
The screening for drought tolerance revealed that 4 isolates were able to grow well on the Luria-Bertani medium containing up to $18 \% \mathrm{NaCl}(w / v)$. Based on the multifunctional properties, twelve isolates were selected to determine the effect on germination and growth on wheat seeds. The outcome of this research proves plausible practical applicability of these PGPB for crop production in soils of saline and arid environments. Keywords: Phosphate solubilization, Plant growth-promoting rbacteria (PGPB), Alkaline phosphatase, Acid phosphatase, Salt stress, Seed germination.

\section{P154. Characterization of Soil Degradation in Northern Morocco}

Abderrahim El Boukhari ${ }^{1 *}$, Mustapha Naimi ${ }^{2}$, Mohamed Chikhaovi ${ }^{2}$, and Damien Raclot ${ }^{1,3}$

1 Institut Agronomique \& Vétérinaire Hassan II, PO. Box. 6608, Madinat Al Irfane, Rabat, Morocco; ${ }^{2}$ Department of Natural Resources \& Environment, Institut Agronomique \& Vétérinaire Hassan II, PO. Box. 6608, Madinat Al Irfane, Rabat, Morocco; ${ }^{3}$ Research Institute for Development (IRD), UMR LISAH, Visiting at Institut Agronomique \& Vétérinaire Hassan II, Rabat, Morocco. "Corresponding and Presenting Author: abderrahimelboukhari@gmail.com.

\section{Abstract}

Water erosion is a major process of soil degradation. In Morocco, it is considered the main cause of desertification. It leads to a reduction of the productive potential of soils and the siltation of dams reservoirs leading to a loss of the volume of mobilizable water and calling into question the durability of these hydraulic structures. Negative effects are reflected in the areas of power generation, drinking water supply and irrigation. Faced with the scale of this scourge, Morocco urgently needs to multiply its interventions to assess the consequences of land degradation and also to develop appropriate conservation programs. In this context, a sample of 15 dams located in the northern region of Morocco was selected to analyze siltation over periods of 8 to 47 years. The bathymetric data were provided by the DRPE (Directorate of Research and Water Planning - State Secretariat for Water), associated with this study. The present paper aims toi) characterize the Specific Degradation (DS) of the northern region of Morocco through the study of 15 watersheds; ii) to deduce a range of variability of DS in this region; and iii) to evaluate the relevance of simple models based on the surface of the basin or the combination of the surface and the anual average water slide, to predict the DS. The approach adopted is based on the confrontation of an updated measure of the Specific Degradation derived from bathymetric surveys with assessments of the latter using established models to predict the siltation of dams in Morocco. Over periods ranging from 8 to 47 years, the review of the siltation of dam reservoirs by analyzing of bathymetric surveys shows an average total annual about $0,6 * 109 \mathrm{~m} 3$, representing a $13 \%$ of the initial total capacity that is of the order of de 4,6*109 m3 and a variability of the specific degradation ranging from 172 to $5365+\mathrm{km}-2$ an-1, whether a ratio of 1 to 31 . Finally, this study shows the very strong variability of the prediction performance of the specific degradation based on simple models using the basin surface and the water slide. Keywords: Water erosion, Dams, Siltation, Specific Degradation.

\section{P155. Interest of the use of Wastewater in Industrial Plants 'Energy Value'. Case of Jatropha curcas}

Ouafae Mokhtari ${ }^{1 *}$, Hassan El Halouani ${ }^{1}$, Kamal Merghem ${ }^{1}$, Imade Hamdani ${ }^{2}$, Abderrahim Lahrach ${ }^{3}$, and Belkhir Hammouti ${ }^{4}$

1 Laboratory of Water Science, Environment and Ecology, Department of Biology, Faculty of Sciences, University Mohammed Premier, Oujda, Morocco; ${ }^{2}$ Laboratory of Applied Analytical Chemistry, Materials and Environment, Faculty of Sciences, University Mohammed Premier, Oujda, Morocco; ${ }^{3}$ Laboratory of Geo-Resources and Environment, Sciences and Technologies Faculty, University Sidi Mohammed Ben Abdallah, Fez, Morocco; ${ }^{4}$ Laboratory of Applied Analytical Chemistry, Materials and Environment, Faculty of Sciences, University Mohammed Premier, Ouida, Morocco. "Corresponding and Presenting Author: mokhtari.ovafae@gmail.com.

\section{Abstract}

To assess the interest of the use of wastewater on the cultivation of Jatropha curcas and the role of the lat- 
ter in the conservation of water and soil and in the production of renewable energy, tests were conducted under semi-arid climatic conditions of northeastern Morocco (Oujda). The aim is to monitor the growth and behavior of Jatropha curcas irrigated with different types of conventional waters and unconventional (including the salinity may be elevated up to $2.82 \mathrm{~mm}$ hos $/ \mathrm{cm}$ ). The performances of growth of Jatropha curcas L. were studied under different types of water of irrigation (wastewater, fertigation, freshwater) and others without irrigation (in rainfall regime). The results showed: (1) The significant effect of wastewater on the vegetative development and yield $(85 \%, p$ $<0.3 \%$ ), (2) Acceleration of the development cycle and formation up to 3 peaks of inflorescences per year, (3) The application of water stress significantly reduced the growth and yield of Jatropha relative to the irrigated plants (a degree of $90 \%, \mathrm{P}<0.2 \%$ ). Its development is slow, and the first flowering is largely delayed ( 8 months) only after the arrival of the first rains of November, (4) The fertigation has improved the performance of Jatropha and accelerated the process of reproduction ( 3 peaks of inflorescences). These results are very similar to those obtained after irrigation with treated wastewater which is only another way of fertigation but much cheaper, and (5) Jatropha curcas shows proper installation despite the high salinity of the soil $(5,89 \mathrm{mmhos} / \mathrm{cm}$ to 7 mmhos / $\mathrm{cm})$. This shows a perfect adaptation of the plant to the different soil types: marginal, skeletal and fertile. The production of Jatropha curcas is closely related to water intake, fertilizers, soil quality and climate. Based on these results, the association of wastewater with Jatropha curcas can be a means of: fight against desertification and erosion, of valorization of the marginal grounds by using Jatropha curcas like a reforestation plant, what will contribute to the sustainable development of the country by the increase in the national heritage in terms of biodiversity, and in terms of renewable energy "a source of green energy". Keywords: Waste waters, fertigation, Jatropha curcas, reforestation, sustainable development.

P156. Development of an in vitro Micropropagation System for Carob (Ceratonia siliqua L.)

Assia Lozzi ${ }^{1 *}$, Rachid Mentag ${ }^{2}$, Rabha Abdelwahd², and Abdelhadi Abousalim ${ }^{1}$
1 Department of Crop Production, Protection and Biotechnology, Institute of Agronomy and Veterinary Medicine Hassan II, Rabat, Instituts-6654, Morocco; ${ }^{2}$ Biotechnology Unit. Regional Center of Agricultural Research. National Institute of Agricultural Research, Rabat- 415, Morocco. "Corresponding and Presenting Author: assia.lozzi@gamail.com.

\section{Abstract}

The carob tree (Ceratonia siliqua L. - Fabaceae), is one of the most important component of Mediterranean vegetation for economic and environmental reasons. The economic importance of this species arises from the industrial use of the locust bean gum obtained from its seeds, which is widely used as a natural additive (E-410) in the food industry to function as a flavor, stabilizer and thickener. The world production of carob pods is estimated at about 156,800 tons/year, the main producers are Spain $(22.8 \%)$, Italy $(20.1$ $\%)$, Morocco (14\%), Portugal (13.9\%) and Turkey (FAO, 2014). In Morocco, a number of ambitious projects promoting the cultivation and exploitation of this genetic resource, have been launched under the Green Morocco Plan. As the traditional carob propagation methods failed to meet the market request, the use of in vitro techniques seems appropriate to fulfil the increased demand for carob plant material. In vitro micropropagation can be applied for massscale production by a commercial outfit and may represent a good alternative methodology to the traditional propagation techniques. During our studies on carob micropropagation, a number of parameters have been investigated to optimize an appropriate protocol for mass propagation of this important plant species. The obtained results are promising and would set a basis for the further development of carob propagation. Keywords: Ceratonia siliqua, carob, micropropagation, in vitro.

\section{P157. Soil Fertility Management In The Irrigated Pe- rimeter of Beni Amir, Morocco}

Houria Dakak ${ }^{1^{*}}$, A. Zouahri', H. Sehlaoui ${ }^{1,3}$, A. Douaik', A. Ghanimi ${ }^{2}$, H. Yachou', and R. Hassikou ${ }^{3}$

${ }^{1}$ National Research Institute of Agricultural Research, CRRAR, Research Unit on the Environment and Conservation of Natural Resources B.P. 6356, Rabat, Mo- 
rocco; ${ }^{2}$ University Mohammed V, Faculty of Sciences, Department of chemistry, Rabat, Morocco; ${ }^{3}$ University Mohammed V, Faculty of Sciences, Department of Biology, Mycology and Environment, Rabat, Morocco. *Corresponding and Presenting Author: Dakak_h@ yahoo.fr.

\section{Abstract}

This work contributes to the rationalization of fertilizer use in order to sustainably manage soil fertility and limit water contamination. Thus, the optimal nitrogen dose was determined on the basis of a calibration test that was installed under the cultivation of soft wheat at the farmer's farm in the Beni Amir region, irrigated area of Tadla. In addition, the statistical analysis showed that the relationship between grain yield and nitrogen dose was parabolic (quadratic), which made it possible to determine the optimal dose (150 kg / ha) which allowed the best grain yield, on the other hand the higher dose $(200 \mathrm{~kg} / \mathrm{ha})$ did not improve grain yield which constitutes a waste of unnecessary money and a risk of pollution by the excess of nitrates which have not been valued for enable better performance. Keywords: Nitrogen fertilization, rationalization, experimentation, optimal dose, wheat culture

P158. Psyttalia concolor (Hymenoptera: Braconidae) Endoparasitoïde of Ceratitis capitata (Diptera: Tephritidae): parasitism rate on Argan ecosystem

Abdelhadi Ajerrar ${ }^{1,2^{*}}$, R. Bouharroud ${ }^{2}, M$ Zaafrani', $^{1}$ B Chebli', A. Amarraque ${ }^{2}$, R. Qessaoui ${ }^{1,2}$, and El H. Mayad $^{3}$

1 Equipe Génie de l'environnement et de biotechnologie, Ecole Nationale des Sciences Appliquée, Agadir, Morocco; ${ }^{2}$ Centre Régional de la Recherche Agronomique d'Agadir, INRA-Morocco; ${ }^{3}$ Faculté des Sciences Ibn Zohr, Agadir, Morocco. "Corresponding and Presenting Author: ajirrar@gmail.com.

\section{Abstract}

The Mediterranean fruit fly, Ceratitis capitata Wiedmann is the most important pest of several fruits plants in the Mediterranean area. Argan trees (Argania spinosa) known as a reservoir of this pest. Psytal- lia concolor is the only Ceratitis capitata's endoparasitoilde known in Morocco. The purpose of this study is to assess the parasitism rate of Ceratitis capitata's endoparasitoïde in Souss region. Fruits sampling were carried out monthly depending on fruit availability. Fruits collected were immediately transported to the laboratory then incubated in ventilated boxes containing sand at $25+/-2{ }^{\circ} \mathrm{C}$ and $70+/-5 \%$ of relative humidity. The results obtained shows that Psyttalia concolor activity's begin early on December when the first ripe fruits appeared, but parasitism rate is very low during December, January and February (less than $1 \%$ ). This rate were gradually increased to reach its maximum during June (6,07\%). Keywords: Ceratitis capitata, endoparasitoid, Argan, Psyttalia concolor, Souss.

\section{P159. Genetic Characterization and Relationship Anal- ysis Among a Collection of Barley Cultivars}

Solange Nyiraguhirwa $\frac{1,2,3,4^{*}}{}$, Zara Grana ${ }^{1,2,3}$, Fatima Henkrar', Hassan Ouabbou', Ibriz Mohammed ${ }^{3}$, Sripada M. Udupa ${ }^{1 \S}$

1 International Center for Agriculture Research in Dry Areas (ICARDA), Rabat, Morocco; 2 Institut National de Recherche Agronomique (INRA) Rabat, Morocco; 3 Ibn Tofail University, Faculty of Sciences, Kenitra, Morocco; 4 Institut National de Recherche Agronomique (INRA), Settat, Morocco. "Presenting author: hirwaso@ gmail.com. §Corresponding author: s.udupa@cgiar. org.

\section{Abstract}

Barley is an important cereal crop in Morocco and is used for animal feed, and human consumption (mainly as bead and malt). Morocco is the 7th largest barley producer in the world. Characterization and deployment of barley genetic diversity is the key for barley improvement. New sources of genetic diversity are being identified and deployed in the barley breeding programs to enhance grain yield, quality, and tolerance to abiotic and biotic stresses. Here we characterize 113 barley accessions from 14 countries: Morocco, Algeria, Tunisia, Egypt, Ethiopia, Libya, Nepal, Italy, Pakistan, Yemen, Syria, Spain, Afghanistan, Mongolia using 14 SSR markers and show that this barley collection is genetically diverse. The 
analysis estimated genetic diversity and PIC values of 0.85 and 0.83 , respectively, and detected 75 alleles indicating high degree of genetic diversity. Both Neighbor joining dendrogram and principal component analysis identified 2 major groups within the analyzed accessions, namely Moroccan accessions vs. others. The results of this study have implications in genetic resources collection, conservation and utilization. Keywords: Genetic diversity, SSR markers, barley, Horedum Vulgare, differents origin.

\section{P160. Assessment of transmission risk of arboviruses in Morocco}

Amal Bennouna ${ }^{1^{*}}$, T. Balenghien ${ }^{2}, H$. Elrhaffouli ${ }^{3}, \mathrm{G}$. $\mathrm{Chlyeh}^{4}$, and O. Fassi Fihri ${ }^{1}$

' Laboratoire de Microbiologie, Département de Pathologie et Santé Publique Vétérinaire, Immunologie, Maladies Contagieuses, Institut Agronomique et Vétérinaire Hassan II, Rabat, Morocco; ${ }^{2}$ UMR Cirad / Inra, Contrôles des Maladies Animales, Montpellier, France; ${ }^{3}$ Laboratoire de Recherche et de Biosécurité P3. Hôpital Militaire d'Instruction Mohamed V de Rabat, Morocco; ${ }^{4}$ Département de Production, Protection et Biotechnologies Végétales. Unité de Zoologie. Institut Agronomique et Vétérinaire Hassan II, Rabat, Morocco. "Corresponding and Presenting Author: amal397@yahoo.fr.

\section{Abstract}

Global changes, including international trade and travelling, have induced a recrudescence of vectorborne diseases, with an important change in their distributions. North Africa is at risk of arbovirus emergence, because it links the Mediterranean basin, where exotic vectors, such as Aedes albopictus, are spreading leading to dengue or chikungunya outbreaks, and the Sahel known as endemic area for some arboviruses such as Rift Valley fever (RVF), which is an acute viral zoonotic disease affecting both humans and domestic animals, and mostly transmitted by mosquitoes. Morocco should be considered as risk of RVF emergence due to its proximity with Mauritania where the disease is endemic. This work aimed to identify potential vectors of Rift Valley fever and to characterize the vector role of Culex pipiens, a species known to carry this disease. We carried out transversal studies across the whole territory, by collecting adults and larvae with a standard protocol in the aim to have an estimation of the species abundance. We carried out adults and larvae collection in more than 140 different sites (urban, suburban and rural) during the springs 2015 and 2016, with a special interest for the South of Morocco, considering the proximity of Mauritania. It is the first recent and extensive prospection of Culicidae in this part of the country. The main abundant collected species were: Culex pipiens, Culex theileri, Culex simpsoni and Culiseta longiareolata. Aedes species could be underestimated due to the timing of collections. However, we identified for the first time in Morocco the Asian tiger mosquito Aedes albopictus. Specimens were identified by both morphology and molecular assay. The population located in Rabat, capital of Morocco, has overwintered during 2015/2016 demonstrating for the first time the establishment of this species in North Africa. We after proceeded at a molecular analysis to characterize the vector role of Culex pipiens by looking for all viral communities harbored by this species. This study is still in process to describe the population of pathogenic arboviruses hosted by Culex pipiens. Keywords: vector-borne diseases, emergence, arboviruses, Culex, Aedes.

\section{P161. Assessment of Flammability of Moroccan Forest Fuels: New Approach to Estimate the Flammability In- dex}

Salaheddine Essaghi ${ }^{1,2, *}$, M'hamed Hachmi $^{2}$, Mohammed Yessef ${ }^{1}$, Mohammed Dehhaoui', and Fahed El Amarty ${ }^{2}$

1 Institut Agronomique et Vétérinaire Hassan II, BP 6202, Rabat-Instituts, Rabat, Morocco; ${ }^{2}$ Ecole Nationale Forestière d'Ingénieurs, BP 511 , Tabriquet, 11015 Salé, Morocco. "Corresponding and Presenting Author: s.essaghi@gmail.com.

\section{Abstract}

A new flammability index (FI) was developed, which integrated two parameters that are highly correlated to fuel moisture content (MC). These parameters are time-to-ignition and flame height. The newly obtained $\mathrm{FI}$-values belong to the variation interval of $\{0 ; 20\}$. In addition to the six flammability classes defined in 
the earlier work, a seventh class (FI > 16.5) was proposed to include fuel species with a high content of volatile flammable-compounds. Flammability testing and $M C$ measurement were performed at a range of $M C$ obtained through a drying process of samples. As a result, FI was statistically highly correlated with $M C$ for all 13 Moroccan forest fuels tested in this study. Following this, linear regression equations were established to predict the FI-value as a function of MC. Therefore, the classification of flammability would depend on the species as well as the MC-value of the samples and the season in which they were collected. Keywords: flammability testing method; flammability classification of fuels; correlation.

\section{P162. Agro-morpho-phenological characterization of an interspecific F3 quinoa population (Chenopodium quinoa Wild)}

Asmaa Allaoui ${ }^{*}$, Eric Nathan Jellen, and Ouafae Benlhabib

IAV Hassan II, Rabat, Morocco. "Corresponding and Presenting Author: ipiapesa@iav.ac.ma.

\section{Abstract}

Multiple biotic and abiotic constraints impact the world food production. According to the FAO, quinoa would be a good ally to fight against hunger and malnutrition for its high nutritional value and its adaptation to harsh agro-ecological zones. This investigation aims to characterize a population of 89 IRL-3 quinoa interspecific lines developed from a natural cross between a Chenopodium quinoa accessions Co407and a wild accession (11-54) (Chenopodium berlandieri) known for its adaptation to high temperatures and its resistance to pests. A Completely Randomized Block Design trial with three replications was conducted between February and July 2017 on an experimental plot at the Agronomic and Veterinary Institute Hassan II of Rabat. Eleven quantitative and six qualitative traits related to the morphology, phenology and production potential were recorded. The analysis of the population descriptors illustrates clearly the large variability of the traits and the accessions. Days to maturity fluctuated between 133 and 170 days, as was the variability of the seed and the inflorescencediameter and color, the plant height, the yield and its components. The analysis of variance (ANOVA) showed highly significant differences among the IRL lines for the studied traits. According to the Pearson Correlation Coefficient, positive and significant relationships were found between the plant height and the length of the inflorescence (0.987) and between the yield and the aerialdry mater (0.812). The PCA showed that the first principal component (PC1) accounted for $71.67 \%$ of the total variance; it is positively associated with the plant height, the inflorescence width and length and the yield. The PC2 accounted for $15.80 \%$ total variability and is the positively correlated with the days to maturity. The dendrogram displays five groups; the first includes 23 low production lines; the second includes early maturing accessions, tall and with long and broad inflorescences; the third includes the a little late to late lines. The fourth group is made of short and unproductive lines and the last brings together medium-sized and medium-yielding lines. Keyswords: Chenopodium quinoa Wild, phenotypic diversity, characterization, genetic diversity.

\section{P163. Effect of Altitude on Pomological and Phyto- chemical Traits of Dallahia Prickly Pear Fruits in North- ern Morocco}

Dramane Y. Koalaga ${ }^{1,2}$, Zerhoune Messaoudi ${ }^{1 *}$, Mohamed Ibriz ${ }^{2}$, and Lhoussain Ait Haddou'

1 Laboratory of Pomology, Department of Arboriculture-Viticulture, National School of Agriculture, B.P. S/40, Meknes, 50001, Morocco; ${ }^{2}$ Laboratory of Genetic and Biometrics, Department of Biology, Faculty of Sciences, Ibn Toufail University, University Campus, BP 133, Kenitra, Morocco. "Corresponding and Presenting Author: messaoudiz@yahoo.fr.

\section{Abstract}

Opuntia sp., commonly known as cactus pear, is originating from Mexico and illustrates the impressive genetic diversity of Cactaceae with 400 species and a great number of varieties for Opuntia ficus indica. Moroccan cactus presents a very high genetic variability and several cultivars exist. They are distinguished by the flowering period (early, late), the flower color (yellow, orange and pink), fruit and pulp colors (green, yellow, orange, red and purple), fruit 
shape (oval, round or oblong), and organoleptic characteristics and antioxidant content of the fruit. Cactus crop plays an important role on the ecological and economical scales but unfortunately, in Morocco, huge amounts of the production are lost due to the lack of valorization prospects. Dallahia prickly pear variety characterized by the green pulp color of the fruit and widely grown in northern Morocco is among the lowest valorized cactus varieties due to the low oil content of its seeds. Therefore, its fruits are mainly used for fresh consumption. The aim of this study is to discuss the effect of the altitude on the pomological (fruit dimensions, seediness and weight) and phytochemical (juiciness, sugariness...) traits of the fruits of this variety in northern Morocco in order to reconsider the different possibilities to transform the excess of the production. Keywords: cactus pear, Opuntia ficus indica, Dallahia, altitude, pomology, HPLC, sugars, organic acids, fibers, gross energy.

\section{P164. Phenotypic Variability of Moroccan Prickly Pear in Southeast Morocco}

Abdelmonaim Hormani Bakali ${ }^{1 *}$, Chakib Alem ${ }^{2}$, Lalla Laaziza Ichir ${ }^{2}$, and El Houssine El Mzouzi ${ }^{3}$

1 National Institute of Agricultural Research, Errachidia, Morocco; ${ }^{2}$ Faculty of Science and Technology of Errachidia, Univer-sity My Ismail, Morocco ; ${ }^{3} \mathrm{Na}$ tional Institute of Agricultural Research, Settat, Morocco. "Corresponding and Presenting Author: homrani_bakali@yahoo.fr.

\section{Abstract}

The genus Opuntia (sensu stricto) has been the subject of various studies in Morocco, but it lacks an assessment the Saharan regions meanly in southeast of Morocco. The overall objective of this work concerns the characterization of Moroccan cactus accessions with a view to their extension to the pre-Saharan ecosystem of Morocco. The phenotypic study of 36 accessions from different regions of Morocco was conducted in the experimental station of INRA Errachidia. Statistical analysis of the data collected included analysis of variance, multivariate analysis and hierarchical classification. These statistical analyzes enabled us to: (i) classify the accessions into four groups and to choose six of them $(77,83,134,135,181,11)$ based on growth and production criteria. (ii) Classify accessions into three categories based on cladode traits, then five accessions with large surface $(36,45,70$, 93). , T3) were chosen. (iii) Make classifications according to the fruit criteria. Thus, the best accessions from the caliber point of view that exceeded on average $120 \mathrm{~g}$ were: 62,149 and 182 . On the other hand, the sweetest ones were: $33,62,70,183$ and T3. This study also includes a comparison between the growths, productions, cladode and fruit traits of the same accessions at Agadir and Errachidia stations. This comparison shows that the characteristics of each accession change according to the biotope. Keywords: Cactus, phenotypic variability, accessions.

\section{P165. Control of Chickpea Leaf Miner (Liriomyza cic- erina R.) Using Planting Dates}

Abdelhadi Sabraoui ${ }^{1,2,3^{*}}$, M. El Bouhssini ${ }^{1}$, S. Lhaloui ${ }^{2}$, A. Bouchelta ${ }^{3}$, and K. El Fakhouri'

${ }^{1}$ International Center for Agricultural Research in the Dry Areas (ICARDA), P.O. Box: 6299- Rabat Institutes, Rabat, Morocco; ${ }^{2}$ Entomology laboratory. Plant protection research unit. Regional Center for Agricultural Research BP 589, Settat, Morocco; ${ }^{3}$ Plant Protection and Environment Laboratory, Moulay Ismail University, B.P. 11201 , Zitoune, Meknes, Morocco. "Corresponding and Presenting Author: a.sabraoui@cgiar. org.

\section{Abstract}

In Morocco, chickpea (Cicer arietinum L.) is the first grain legume in the crop rotation system after cereals, occupying $45.12 \%$ of food legumes area and producing around 441000 tonnes in 2016. However, the chickpea yield is still low and this is due to many biotic stresses. The chickpea leaf miner (Liriomyza cicerina R.) is one of the main insect pests damaging chickpea in North Africa and the Mediterranean region. To determine which planting date will be optimum for the chickpea crop to reduce Leaf miner infestation and losses caused by this pest, an experiment using four improved kabouli varieties planted in winter and spring, treated/untreated with insecticide, was carried out at two locations in Morocco. The percent infestation by the leaf miner was about $25 \%, 43$ $\%$ and $3 \%$ for untreated winter planting, untreated 
spring planting and the treated plots, respectively. The grain yield losses caused by this pest were on average $42 \%$ for the spring-sown crop and $20 \%$ for winter planting. The use of winter planting date combined with biological insecticides could be included as options of integrated pest management for the control of chickpea leaf miner. Keywords: Planting date, Leaf miner, Chickpea, yield losses.

\section{P166. In Vitro Evaluation of Compost Extracts Efficiency as Biocontrol Agent of Date Palm Fusarium Wilt}

El Kinany Said ${ }^{1,2,3^{*}}$, Achbani El hassan ${ }^{2}$, Haggoud Abdellatif ${ }^{3}$, Ibijbijen Jamal ${ }^{4}$, Belmalha Saadia', Rachidi Fouad', Echchgadda Ghizlane', and Bouamri Rachid'

1 Department of Plant and Environment Protection, National School of Agriculture, Meknes, Morocco; ${ }^{2}$ Laboratory of Plant Protection URPP, National Institute for Agricultural Research, Meknes, Morocco; ${ }^{3}$ Laboratory of Microbial Biotechnology, Faculty of Sciences and Technology, Sidi Mohamed Ben Abdellah University, Fez, Morocco; 4 Department of Biology, Faculty of Sciences, Moulay Ismail University, Meknes, Morocco. "Corresponding and Presenting Author: rbouamri@ enameknes.ac.ma. or said.elkinany@usmba.ac.ma.

\section{Abstract}

Bayoud disease, vascular wilt of date palm caused by Fusarium oxysporum f. sp. albedinis (Foa), is widely distributed in all date palm growing regions of Morocco. It is the most serious disease of the date palm. Resistance to the pathogen was found in a few cultivars of date palm, which unfortunately produce poorquality fruit. Compost is recognized for their ability to improve soil characteristics and to protect the crops against biotic and abiotic stress. In this experiment, in vitro effects of different concentrations of sterilized and unsterilized compost extract on the growth of $F$. oxysporum f. sp. albedinis were evaluated to control Bayoud disease. All concentration of unsterilized compost extract significantly decreased radial growth of Foa. In fact, fungal radial growth inhibition ranged from 20 to $97 \%$ depending concentrations. Higher antifungal activities were noted in 30 and $40 \%$ concentration (more than 93\%). Nevertheless, sterile compost extract inhibited mycelia growth only for the $40 \%$ concentration with $18 \%$ fungal growth inhibition, while lower concentrations were not effective. This experiment constitutes a biological approach to protect date palm groves in oasis ecosystem in Morocco. Keywords: Date palm, Fusarium oxysporum f. sp. albedinis, compost extract, mycelium growth, inhibition rate.

\section{P167. Study of Olive Fruit Peroxidase Activity Against Bactrocera oleae Attack in Attaovia Region, Haouz of Morocco}

Wassila Arkize ${ }^{*}$, M. A. El Alaoui El Fels², K. Naamani $^{2}$, and A. Elkeroumi ${ }^{3}$

1 Laboratoire Biotechnologie et valorisation des ressources végétales, Faculté des Sciences semlalia Marrakech, Morocco; ${ }^{2}$ Laboratoire Biotechnologie et valorisation des ressources végétales, Faculté des Sciences semlalia Marrakech, Morocco; ${ }^{3}$ Laboratoire Biotechnologie et valorisation des ressources végétales, Faculté des Sciences semlalia Marrakech, Morocco. ${ }^{*}$ Corresponding and Presenting Author: wassila. arkize@gmail.com.

\section{Abstract}

Bactrocera oleae (Rossi) is a pest considered as a major biotic stress of olive fruit in Morocco as well as other Mediterranean countries. Bactrocera oleae is causing considerable damages on the quality of table olives due to laying pits, but also on the olive oil production due to the olive fruit oxidation which results from the consumption of the olive fruit pulp by Bactrocera oleae's larvae. Plants can defend against the pathogens like Bactrocera oleae by synthesizing peptides and defense proteins, among these the PR proteins, Among PR proteins are peroxidase which have several functions of plant protection against pathogens. Peroxidases catalyzes the reduction of $\mathrm{H} 2 \mathrm{O} 2$ from using different molecules such as phenolic compounds, precursors of the lignin and other auxins, indeed peroxidase can cope with biotic stress by strengthening the cell walls or producing active forms of oxygen. Olive fruits were sampled from ATTAOUIA region, HAOUZ of Morocco, in twoo sites about $24 \mathrm{~km}$ apart, whitch differes in irrigation mode (Site Saguia: Irrigated and Site Bour: not irrigated). The purpose of this study is to compare peroxidasic activity in infected and not infected olive fruits in both of study sites. Keywords: Bactrocera oleae, olive fruit, Peroxidasic 
activity, PR proteins.

P168. Interest of the use of Wastewater in Industrial Plants 'Energy Value'. Case of Jatropha curcas

Ouafae Mokhtari ${ }^{*}$, Hassan El Halouani ${ }^{1}$, Kamal Merghem ', Imade Hamdani ${ }^{2}$, Abderrahim Lahrach ${ }^{3}$, and Belkhir Hammouti $^{4}$

Laboratory of Water Science, Environment and Ecology, Department of Biology, Faculty of Sciences, University Mohammed Premier, Oujda, Morocco; ${ }^{2}$ Laboratory of Applied Analytical Chemistry, Materials and Environment, Faculty of Sciences, University Mohammed Premier, Oujda, Morocco; ${ }^{3}$ Laboratory of Geo-Resources and Environment, Sciences and Technologies Faculty, University Sidi Mohammed Ben Abdallah, Fez, Morocco; ${ }^{4}$ Laboratory of Applied Analytical Chemistry, Materials and Environment, Faculty of Sciences, University Mohammed Premier, Oujda, Morocco. "Corresponding and Presenting Author: mokhtari.ovafae@gmail.com.

\section{Abstract}

To assess the interest of the use of wastewater on the cultivation of Jatropha curcas and the role of the latter in the conservation of water and soil and in the production of renewable energy, tests were conducted under semi-arid climatic conditions of northeastern Morocco (Oujda). The aim is to monitor the growth and behavior of Jatropha curcas irrigated with different types of conventional waters and unconventional (including the salinity may be elevated up to $2.82 \mathrm{~mm}$ hos $/ \mathrm{cm}$ ). The performances of growth of Jatropha curcas L. were studied under different types of water of irrigation (wastewater, fertigation, freshwater) and others without irrigation (in rainfall regime). The results showed: (1) The significant effect of wastewater on the vegetative development and yield $185 \%, \mathrm{p}$ $<0.3 \%$ ), (2) Acceleration of the development cycle and formation up to 3 peaks of inflorescences per year, (3) The application of water stress significantly reduced the growth and yield of Jatropha relative to the irrigated plants (a degree of $90 \%, \mathrm{P}<0.2 \%$ ). Its development is slow, and the first flowering is largely delayed ( 8 months) only after the arrival of the first rains of November, (4) The fertigation has improved the performance of Jatropha and accelerated the process of reproduction (3 peaks of inflorescences). These results are very similar to those obtained after irrigation with treated wastewater which is only another way of fertigation but much cheaper, and (5) Jatropha curcas shows proper installation despite the high salinity of the soil $(5,89 \mathrm{mmhos} / \mathrm{cm}$ to 7 mmhos / $\mathrm{cm})$. This shows a perfect adaptation of the plant to the different soil types: marginal, skeletal and fertile. The production of Jatropha curcas is closely related to water intake, fertilizers, soil quality and climate. Based on these results, the association of wastewater with Jatropha curcas can be a means of: fight against desertification and erosion, of valorization of the marginal grounds by using Jatropha curcas like a reforestation plant, what will contribute to the sustainable development of the country by the increase in the national heritage in terms of biodiversity, and in terms of renewable energy "a source of green energy". Keywords: Waste waters, fertigation, Jatropha curcas, reforestation, sustainable development.

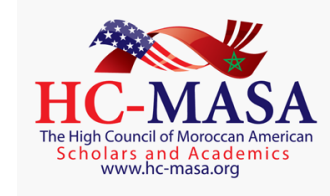

The International American Moroccan Agricultural Sciences Conference (AMAS Conference; www.amas-conference.org) is an international conference organized by the High Council of Moroccan American Scholars and Academics (HC-MASA; www.hc-masa.org). 